\title{
Quantifizierung des Luftvolumenstromes in frei gelüfteten Rinderställen mit Hilfe der Kompartimentalisierungsmethode zur Bestimmung umweltrelevanter Emissionsmassenströme
}

\author{
Dissertation \\ zur Erlangung des Doktorgrades \\ der Fakultät für Agrarwissenschaften \\ der Georg-August-Universität Göttingen
}

vorgelegt von

Dipl.-Ing. agr. Gunnar Brehme

geboren in Halle/Saale

Göttingen, November 2000 


\section{7}

1. Referent: Prof. Dr. Ir. H. Van den Weghe

2. Referent: Prof. Dr.-Ing. A. Munack

Tag der mündlichen Prüfung: 23. November 2000

(c) 2000 im Selbstverlag, Dr. sc. agr. Gunnar Brehme

Bezugsquelle:

http://webdoc.sub.gwdg.de/diss/2001/brehme/index.html

Alle Rechte vorbehalten. 


\section{Vorwort}

Die Dissertation ist während der Beteiligung am BML-Forschungsprojekt zur Erfassung der Emissionen und Immissionen aus der Rinderhaltung entstanden. In der Rinderhaltung dominieren Ställe mit natürlicher Lüftung. Unabhängig von der Gestaltung der jeweiligen Lüftungsform wird die räumliche und zeitliche Variabilität der Luftvolumenströme im Wesentlichen durch die atmosphärische Turbulenz geprägt. Dabei hat neben der Windgeschwindigkeit vor allem die Windrichtung einen entscheidenden Einfluss auf die stallinternen Strömungsverhältnisse und damit auf die Stofffreisetzungsvorgänge im System. Die Unkenntnis dieser stallinternen Strömungsvorgänge führt zu erheblichen Diskrepanzen in der Methodik der Bestimmung von Luftvolumenströmen.

Ausgehend von den elementaren Grundlagen der Strömungsmechanik wurde ein Berechnungsverfahren entwickelt, das es erlaubt, eine Vorstellung von den Luftaustauschvorgängen in Nutztierställen mit freier Lüftung zu erhalten. Das Verfahren beruht dabei auf Messwerten, die in der Praxis erhoben wurden. Zur Auswertung wird die sog. Kompartimentalisierungsmethode verwendet, die bereits seit Jahrzehnten im medizinischen Bereich eingesetzt wird. Das Prinzip besteht darin, komplexe Stoffaustauschvorgänge in vereinfachenden Bilanzierungsgleichungen darzustellen, ohne dass die grundsätzlichen Zusammenhänge verloren gehen.

Die verbesserte Berechnung der Luftvolumenströme führt zu fundierten und detaillierten Aussagen bezüglich des Umfangs von Geruchseinträgen in den Nahbereich von Tierställen. Diese Geruchseinträge haben eine große Bedeutung für die Umwelt und damit im Rahmen von Genehmigungsverfahren.

Vechta, im November 2000

Prof. Dr. Ir. H. Van den Weghe 
Meiner lieben Kati 


\section{Abkürzungs- und Symbolverzeichnis}

\begin{tabular}{|c|c|c|}
\hline$A$ & {$\left[m^{2}\right]$} & Fläche \\
\hline$A r$ & - & Archimedes-Zahl \\
\hline$A T C$ & - & Austausch der Konzentration \\
\hline $\mathrm{a}$ & {$\left[\mathrm{m}^{3} / \mathrm{s}\right]$} & Austauschkoeffizient \\
\hline $\mathrm{a}_{i j}$ & - & Austausch \\
\hline BImSchG & - & Bundesimmissionsschutzgesetz \\
\hline BML & - & Bundesministerium für Ernährung, Landwirtschaft und Forsten \\
\hline $\mathrm{b}$ & - & Ergebnisvektor \\
\hline$C$ & {$\left[\mathrm{~kg} / \mathrm{m}^{3}\right]$} & Gaskonzentration \\
\hline $\bar{C}$ & {$\left[\mathrm{~kg} / \mathrm{m}^{3}\right]$} & mittlere Gaskonzentration \\
\hline$c^{\prime}$ & {$\left[k g / m^{3}\right]$} & Schwankungsteil der Konzentration \\
\hline$c_{p}$ & - & (Wind-)Druckkoeffizient \\
\hline CFD & - & Computational Fluid Dynamics \\
\hline $\mathrm{CH}_{4}$ & - & Methan \\
\hline $\mathrm{CO}_{2}$ & - & Kohlendioxid \\
\hline c.p. & - & ceteris paribus \\
\hline$c_{W}$ & - & Widerstandsbeiwert \\
\hline $\mathrm{D}$ & - & Diffusion \\
\hline$D_{m}$ & {$\left[\mathrm{~m}^{2} / \mathrm{s}\right]$} & molekularer Diffusionskoeffizient (Ficksches Gesetz) \\
\hline$D_{x x}$ & {$\left[\mathrm{~m}^{2} / \mathrm{s}\right]$} & Diffusionskoeffizient \\
\hline DOAS & - & Differentielle Optische Absorptionsspektroskopie \\
\hline$E$ & - & Erwartungswert \\
\hline$E_{A}$ & - & Eulersche Zahl \\
\hline $\mathrm{e}_{i j}$ & - & Eintrag \\
\hline$F$ & {$[k g]$} & Produktionsrate des Tracers (Quellterm) \\
\hline FTIR & - & Fourier Transformations Infrarot Spektrometrie \\
\hline GC & - & Gaschromatograph \\
\hline GE & - & Geruchseinheit \\
\hline GV & - & Großvieheinheit (500 kg) \\
\hline$g$ & {$\left[\mathrm{~m} / \mathrm{s}^{2}\right]$} & Erdbeschleunigung \\
\hline $\mathrm{H}_{2} \mathrm{O}$ & - & Wasser \\
\hline$h$ & {$[m]$} & Höhe \\
\hline$h_{e}$ & {$[J / k g L u f t]$} & spezifische Enthalpie der Luft \\
\hline IR & - & Infrarot \\
\hline$i, j, k$ & - & Laufindizes \\
\hline
\end{tabular}




\begin{tabular}{|c|c|c|}
\hline $\mathrm{K}$ & - & Konvektion \\
\hline $\mathrm{k}$ & {$\left[m^{3} / h \cdot(P a)^{\frac{2}{3}}\right]$} & Durchflusskoeffizient \\
\hline$k n$ & - & Knoten \\
\hline LG & {$[k g]$} & Lebendgewicht \\
\hline LPG & - & Landwirtschaftliche Produktionsgenossenschaft \\
\hline$M$ & - & Matrix der Austauschkoeffizienten \\
\hline$m$ & {$[k g]$} & Masse \\
\hline$\dot{m}$ & {$[\mathrm{~kg} / \mathrm{s}]$} & Massenstrom \\
\hline$m a$ & - & Masche \\
\hline $\mathrm{N}_{2} \mathrm{O}$ & - & Distickstoffoxid (Lachgas) \\
\hline NDIR & - & Nicht Dispersive Infrarot Spektrometrie \\
\hline $\mathrm{NH}_{3}$ & - & Ammoniak \\
\hline NO & - & Stickstoffmonoxid \\
\hline $\mathrm{NO}_{2}$ & - & Stickstoffdioxid \\
\hline$N O_{x}$ & - & Stickoxide \\
\hline$n^{\prime}$ & - & Normalenrichtung \\
\hline$n_{S}$ & - & Strömungsexponent \\
\hline$O_{3}$ & - & Ozon \\
\hline$p$ & {$[P a]$} & Druck \\
\hline$p p b$ & - & parts per billion \\
\hline ppm & - & parts per million \\
\hline ppt & - & parts per trillion \\
\hline$p_{U}$ & {$[P a]$} & dynamischer Druck \\
\hline$p_{W}$ & {$[P a]$} & statischer Druck \\
\hline $\mathrm{Q}$ & - & Quelle \\
\hline$q$ & {$\left[W / m^{2}\right]$} & Wärmestromdichte \\
\hline$R$ & - & Residuum, Approximationsfehler \\
\hline$R e$ & - & Reynolds-Zahl \\
\hline $\mathrm{S}$ & - & Senke \\
\hline$S F_{6}$ & {$\left[\mathrm{~kg} / \mathrm{m}^{3}\right]$} & Schwefelhexafluorid \\
\hline StA & {$[B q]$} & Strahlungsaktivität (in Becquerel) \\
\hline$T$ & {$\left[{ }^{\circ} \mathrm{C}\right]$} & Temperatur \\
\hline TA-Luft & - & Technische Anleitung zur Reinhaltung der Luft \\
\hline$t$ & {$[s]$} & Zeit \\
\hline $\mathrm{U}$ & - & Umwandlung \\
\hline UV & - & Ultraviolett \\
\hline$V$ & {$\left[m^{3}\right]$} & Volumen \\
\hline
\end{tabular}




$\begin{array}{lll}\dot{V} & {\left[\mathrm{~m}^{3} / \mathrm{s}\right]} & \text { Volumenstrom } \\ \text { VDI } & - & \text { Verein Deutscher Ingenieure } \\ \overrightarrow{\vec{V}} & {[\mathrm{~m}]} & \text { zeitlicher Mittelwert des Ortsvektors der Geschwindigkeit } \\ \overrightarrow{V_{\text {diff }}} & {[\mathrm{m}]} & \text { Ortsvektor der diffusen Geschwindigkeit } \\ \mathrm{x} & - & \text { Lösungsvektor } \\ x_{f} & {[\mathrm{~g} / \mathrm{kgLuft}]} & \text { Fremdstoffgehalt } \\ x_{s} & {[\mathrm{~g} / \mathrm{kgLuft}]} & \text { Wasserdampfgehalt } \\ v & {[\mathrm{~m} / \mathrm{s}]} & \text { Geschwindigkeit } \\ v^{\prime} & {[\mathrm{m} / \mathrm{s}]} & \text { Schwankungsteil der Geschwindigkeit } \\ \vec{v}(x, y, z) & {[\mathrm{m}]} & \text { Ortsvektor der Geschwindigkeit }(=u \vec{i}+v \vec{j}+w \vec{k}) \\ \vec{w} & - & \text { Wichtungsfunktion } \\ x, y, z & {[\mathrm{~m}]} & \text { Ortskoordinaten } \\ Z D & - & \text { Zellendifferenz } \\ Z S & - & \text { Zeitschritt }\end{array}$

griechische Symbole

$\begin{array}{lll}\alpha & {[1 / s]} & \text { Luftwechselkoeffizient } \\ \bar{\alpha} & {[1 / \mathrm{s}]} & \text { mittlerer Luftwechselkoeffizient } \\ \varepsilon_{C} & - & \text { Messfehler bei der Konzentrationsbestimmung } \\ \varepsilon_{\dot{V}} & - & \text { Messfehler bei der Luftvolumenstrombestimmung } \\ \varepsilon_{R} & - & \text { Messfehler bei der Raumvolumenbestimmung } \\ O & - & \text { Ordnung } \\ \rho & {\left[\mathrm{kg} / \mathrm{m}^{3}\right]} & \text { Dichte } \\ v & {\left[\mathrm{~m}^{2} / \mathrm{s}\right]} & \text { kinematische Viskosität } \\ \Phi & {\left[\mathrm{m}^{2} / \mathrm{s}\right]} & \text { Dissipation der kinetischen Energie }\end{array}$

spezielle Symbole

$\begin{array}{lll}\frac{d}{d t} & - & \text { substanzielle Ableitung } \\ \operatorname{div} \vec{v} & \frac{\partial u}{\partial x}+\frac{\partial v}{\partial y}+\frac{\partial w}{\partial z} & \text { Divergenz } \\ \operatorname{grad} C & \frac{\partial C}{\partial x}, \frac{\partial C}{\partial y}, \frac{\partial C}{\partial z} & \text { Gradient } \\ \nabla & \frac{\partial}{\partial x}, \frac{\partial}{\partial y}, \frac{\partial}{\partial z} & \text { Nabla-Operator } \\ \triangle & \frac{\partial^{2}}{\partial x^{2}}, \frac{\partial^{2}}{\partial y^{2}}, \frac{\partial^{2}}{\partial z^{2}} & \text { Laplace-Operator } \\ \oslash & - & \text { Durchschnitt } \\ \circ & \frac{\partial}{\partial t} & \text { zeitliche Ableitung } \\ - & - & \text { Mittelwertbildung }\end{array}$

Hier nicht aufgeführte Formelzeichen sind entweder allgemein bekannt oder im Text erläutert. 


\section{Inhaltsverzeichnis}

1 Einführung und Problemstellung $\quad 13$

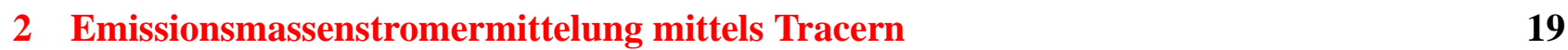

2.1 Luftvolumenstromermittelung bei homogener Tracerverteilung im Raum . . . . . . . 19

2.1 .1 Abklingmethode . . . . . . . . . . . . . . . 20

2.1.2 Methode der konstanten Tracerinjektion . . . . . . . . . . . . . . . . . 22

2.1.3 Methode der variablen Tracerinjektion . . . . . . . . . . . . . 23

2.1.4 Unzulänglichkeiten bei der Mittelwertbildung und Sensorpositionierung . . . 24

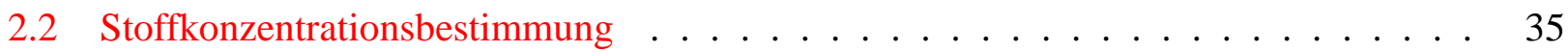

3 Quantitative Erfassung von Raumluftströmungen 37

3.1 Experimentelle Vorgehensweise . . . . . . . . . . . . . . . 37

3.2 Mathematisch-numerische Vorgehensweise . . . . . . . . . . . . 37

3.2.1 Einphasenströmung . . . . . . . . . . . . . . . . 39

3.2.2 Zweiphasenströmung . . . . . . . . . . . . . . . . . 41

3.3 Numerische Strömungssimulation . . . . . . . . . . . . . . . 45

3.3.1 Finite Differenzen Verfahren . . . . . . . . . . . . . . . . 46

3.3.2 Finite Volumen Verfahren . . . . . . . . . . . . . . . . . 47

3.3 .3 Finite Elemente Verfahren . . . . . . . . . . . . . . . . 50

3.4 Skalare Lösungsverfahren . . . . . . . . . . . . . . . . . . . . . 50

3.4.1 Druckdifferenzmessungen ..................... 51

3.4.2 Maschenverfahren ......................... 54

4 Methode der Kompartimentalisierung räumlicher Systeme 56

4.1 Definition und Anwendungsbereiche . . . . . . . . . . . . . . 56

4.2 Allgemeine Vorgehensweise . . . . . . . . . . . . . . 58

4.3 Entwicklung der Systemgleichungen . . . . . . . . . . . . . . . . . . 60

4.3.1 Herleitung des linearen Gleichungssystems . . . . . . . . . . . . . 62

4.3.2 Endgültiges Gleichungssystem . . . . . . . . . . . . . . . . . . 67

4.4 Unterschied zur Abklingmethode . . . . . . . . . . . . . . . . 72 
5 Material $\quad \mathbf{7 4}$

5.1 Ställe im Feldversuch . . . . . . . . . . . . . . . . . . . . . 74

5.2 Ablauf der Tracergasmessung . . . . . . . . . . . . . . . 77

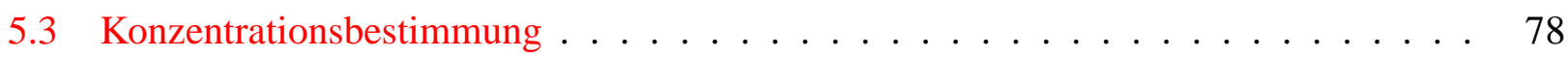

5.4 Software und Programmablauf . . . . . . . . . . . . . . . . . 80

$\begin{array}{llr}6 & \text { Ergebnisse } & 84\end{array}$

6.1 Modellversuche . . . . . . . . . . . . . . . . . . . . 84

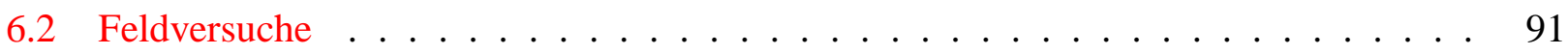

7 Diskussion $\quad 101$

8 Zusammenfassung $\quad 110$

9 Summary 113

10 Anhang $\quad 121$ 


\section{Abbildungsverzeichnis}

Abb. 1: Ansicht eines Offenfrontstalles . . . . . . . . . . . . . . . . . . 13

Abb. 2: Zeitliche Massenänderung zwischen zwei Flächen . . . . . . . . . . . . . 16

Abb. 3: Gängige Stalltypen der Zwangslüftung und der freien Lüftung . . . . . . . . . . 17

Abb. 4: Geschwindigkeitsfeld im zweidimensionalen Raumquerschnitt (2 m x 2 m) . . . 24

Abb. 5: Unterschied zwischen Numerik und Messtechnik . . . . . . . . . . . . . . . 26

Abb. 6: Ansprechzeit der Tracerkonzentration und Luftwechselkoeffizienten . . . . . . . . 27

Abb. 7: Besatz eines Raumes mit unterschiedlicher Sensorzahl _ . . . . . . . . . . . 28

Abb. 8: Höhe der Luftwechselkoeffizienten bei unterschiedlicher Sensorzahl . . . . . . . . 28

Abb. 9: Abklingkurven der Tracerkonzentration an neun Messpunkten . . . . . . . . . . . 29

Abb. 10: Verlauf der Abklingkurven an den Messpunkten 3, 5 und 7 . . . . . . . . . 30

Abb. 11: Geschwindigkeits- und Konzentrationsprofil in einem Traufen-First-Stall . . . . . 32

Abb. 12: Konzentrationsprofile in einem Traufen-First-Stall . . . . . . . . . . . . . . . . 34

Abb. 13: Durchströmung eines Volumens V mit der Oberfläche A . . . . . . . . . . 39

Abb. 14: Laminare und turbulente Strömung . . . . . . . . . . . . . . . . . . 43

Abb. 15: Finite Differenzen Verfahren . . . . . . . . . . . . . . . . 46

Abb. 16: Finite Volumen Verfahren . . . . . . . . . . . . . . . . . . . 48

Abb. 17: Lage der Neutralen Zone bei verschiedenen Windgeschwindigkeiten . . . . . . 51

Abb. 18: Stoffströme durch ein Kompartiment . . . . . . . . . . . . . . . . . . 59

Abb. 19: Aufteilung des Systems Nutztierstall in vier Teilbereiche . . . . . . . . . . . 60

Abb. 20: Übergang zu definierten Kompartimentvolumina im Idealfall . . . . . . . . . . . 61

Abb. 21: Stall- und Umgebungskompartimente . . . . . . . . . . . . . . . . . 62

Abb. 22: Austauschgrößen der Konzentration (ATC) f . . . . . . . . . . . . 63

Abb. 23: Austausch eines Skalars über eine Grenzfläche . . . . . . . . . . . . . 64

Abb. 24: Mögliche Austauschkoeffizienten eines quaderförmigen Kompartimentes . . . 66

Abb. 25: Unterschied zwischen Kompartimentalisierung und Abklingmethode . . . . . . . 72

Abb. 26: Südwestansicht eines Offenstalles in Faulenhorst (Sachsen-Anhalt) . . . . . . . . 74

Abb. 27: Prinzipielle Vorgehensweise bei der Kompartimentalisierung in Praxisställen . 75 
Abb. 28: Lage und Anordnung der Kompartimente (Faulenhorst, Sachsen-Anhalt) . . . . . 75

Abb. 29: Südliche Längsseite eines Offenfrontstalles in Rothenstadt (Bayern) . . . . . . 76

Abb. 30: Lage und Anordnung der Kompartimente (Rothenstadt, Bayern) . . . . . . . . 77

Abb. 31: Aufbau des photoakustischen Gasanalysators 1302 . . . . . . . . . . . . . 79

Abb. 32: Haupteingabemaske . . . . . . . . . . . . . . . . . . . . . 81

Abb. 33: Programmablaufplan (PAP) . . . . . . . . . . . . . . . 83

Abb. 34: Geschwindigkeitsfeld $(2 \mathrm{~m}$ x $2 \mathrm{~m} ; 9$ Kompartimente $) \ldots \ldots$. . . . . . . 85

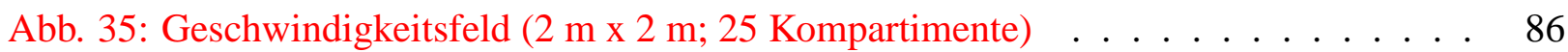

Abb. 36: Geschwindigkeitsfeld ( $2 \mathrm{~m}$ x 2 m; 9 Kompartimente mit Hindernis $) \quad \ldots . .87$

Abb. 37: Geschwindigkeitsfeld (2 m x 2 m; 9 Kompartimente; zwei Auslässe) . . . . . . . 88

Abb. 38: Verlauf Luftvolumenstrom Faulenhorst . . . . . . . . . . . . . . . . . 91

Abb. 39: Ergebnisse Stall Faulenhorst . . . . . . . . . . . . . . . . . . 92

Abb. 40: Mittlere Luftwechselkoeffizienten in Faulenhorst . . . . . . . . . . . . . . . 93

Abb. 41: Verlauf Massenstrom Faulenhorst . . . . . . . . . . . . . . . 95

Abb. 42: Verlauf Luftvolumenstrom Rothenstadt _ . . . . . . . . . . . . . . . . . 96

Abb. 43: Ergebnisse Stall Rothenstadt . . . . . . . . . . . . . . . . . . . . . . 97

Abb. 44: Mittlere Luftwechselkoeffizienten in Rothenstadt . . . . . . . . . . . . . . . 98

Abb. 45: Verlauf Massenstrom Rothenstadt . . . . . . . . . . . . . . . . . . . 100 


\section{Tabellenverzeichnis}

Tab. 1: Luftwechselkoeffizienten für verschiedene Kurvenabschnitte . . . . . . . . . . . 30

Tab. 2: Ergebnisübersicht Modellversuche . . . . . . . . . . . . . . . . . . 89

Tab. 3: Konzentrationen im Stall Faulenhorst . . . . . . . . . . . . . . . . . . . 94

Tab. 4: Massenströme im Stall Faulenhorst . . . . . . . . . . . . . . . . . . . . . . . 94

Tab. 5: Konzentrationen im Stall Rothenstadt . . . . . . . . . . . . . . . . . . . 99

Tab. 6: Massenströme im Stall Rothenstadt _ . . . . . . . . . . . . . . . . . . . . . 99

Tab. 7: Errechnete Luftvolumenströme aller Modellversuche . . . . . . . . . . . . . . 122

Tab. 8: Errechnete Luftvolumenströme aller Feldversuche . . . . . . . . . . . . . . . 123

Tab. 9: Konzentrationsmesswerte Modellversuch (9 Kompartimente) . . . . . . . . . . . . 124 


\section{Einführung und Problemstellung}

Die landwirtschaftliche Nutztierhaltung vollzieht sich überwiegend in geschlossenen oder offenen Gebäuden. Dies führt in der Regel zu einer Reihe stofflicher Emissionen (Gas, Staub, Keime) sowie zur Freisetzung von Geruchsstoffen. Geruchsstoffe bewirken beim Menschen über Reizmechanismen die Wahrnehmung "Geruch”. Gasförmige Emissionen entstehen primär durch den intermediären Stoffwechsel (z.B. $\mathrm{CO}_{2}, \mathrm{CH}_{4}$ ) und/oder aus den Ausscheidungen und sekundär nachgeschalteten Reaktionen in den Exkrementen (z.B. $\mathrm{NH}_{3}, \mathrm{~N}_{2} \mathrm{O}$ ).

Der Transport und die Ausbreitung luftgetragener Substanzen wird als Transmission bezeichnet. Die Transmission ist abhängig von der örtlichen Topographie und den atmosphärischen Bedingungen wie Windgeschwindigkeit und Windrichtung. Das letzte Glied der Kausalitätskette ist die Immission. Als Immissionsort wird der Ort bezeichnet, an dem sich die emittierten Substanzen bemerkbar machen. Ein wichtiges Beispiel hierfür stellt der Geruchseintrag in die nähere Umgebung von Ställen dar, der in zahlreichen Situationen als belästigend empfunden wird.
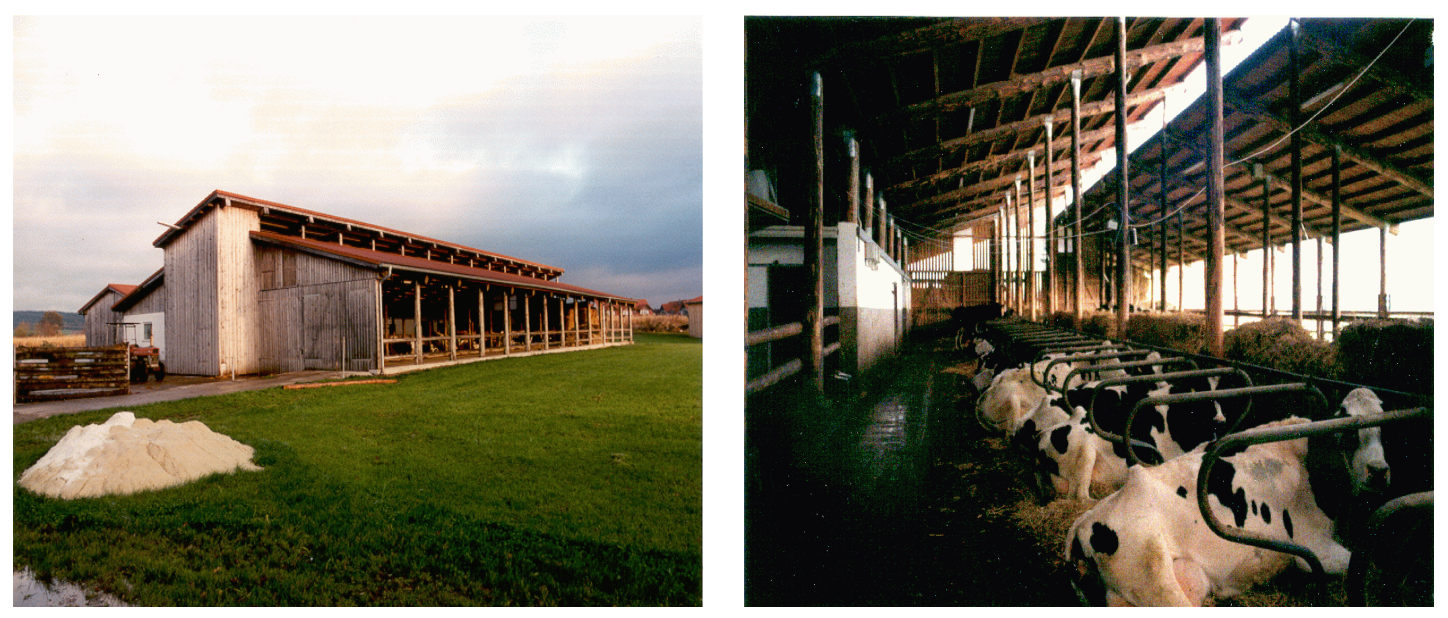

Abbildung 1: Ansicht eines Offenfrontstalles. Die beiden Giebelseiten sind als Spaceboard ausgelegt; die der offenen Seite gegenüber befindliche Rückwand weist einen durchgehenden Öffnungsschlitz von $0,5 \mathrm{~m}$ in Traufhöhe auf.

Ziel des Luftwechsels in Ställen ist es u.a., die Tiere mit Frischluft zu versorgen. Ferner sollen Keime, Stäube sowie Wärmelasten aus dem Stall abtransportiert werden, um zum einen die Tiergesundheit nicht zu gefährden und zum anderen die Nachbarschaft keinen unzumutbaren Belästigungen auszusetzen.

Um das Strömungsgeschehen im Stall zu eruieren, soll der Luftmassenstrom, der durch das Stallsystem bewegt wird, bestimmt werden. Die Luft gilt als Trägermedium für die eingangs angesprochenen Gase, Stäube, Keime und Gerüche. Diese Freisetzungen werden z.B. als Konzentrationen (Dichten) 
luftfremder Stoffe gekennzeichnet. Für das Gesamtgemisch, bestehend aus den Anteilen $i=1 \ldots n$, gilt mit

$m_{\text {ges }}:$ Gesamtmasse

$m_{1}$ : Massetrockener Luft

$m_{2}:$ Masse Wasserdampf

$m_{n}:$ Masse Fremdbeimengungen

die folgende Gleichung 1

$$
\frac{m_{1}}{m_{\text {ges }}}+\frac{m_{2}}{m_{\text {ges }}}+\ldots+\frac{m_{n}}{m_{\text {ges }}}=1
$$

und weiter

$$
\begin{aligned}
m_{\text {ges }} & =m_{1}+m_{2}+\ldots+m_{n} \\
& =m_{1}\left(1+\frac{m_{2}}{m_{1}}+\ldots+\frac{m_{n}}{m_{1}}\right)
\end{aligned}
$$

Bei gesättigter Luft enthält $1 \mathrm{~kg}$ Trockenmasse Luft die Wasserdampfmasse $\left(x_{s}=\frac{m_{2}}{m_{1}}\right) x_{s} \cdot m_{1}$, mit $x_{s}$ als Wasserdampfgehalt der Luft. Mit $x_{f}=\frac{m_{n}}{m_{1}}$ als Fremdstoffgehalt ergibt sich aus Gleichung 3

$$
m_{\text {ges }}=m_{1}\left(1+x_{s}+\ldots+x_{f}\right)
$$

Der Anteil der Wasserdampf- und Fremdstoffmassen kann mit $O\left(10^{-3}\right)$ bzw. $O\left(10^{-6}\right)$ vernachlässigt werden.

Bezieht man nun unter Annahme der Gleichungen 1 und 4 den luftseitigen Massenstrom $\dot{m}_{L u f t}$ auf die Dichte der Luft $\rho_{\text {Luft }}$ und den Massenstrom $\dot{m}_{\text {Luftfremd }}$ aller luftfremden Stoffe auf die entsprechende Dichte der luftfremden Stoffe $\rho_{\text {Luftfremd }}$, dann erhält man den sog. Luftvolumenstrom $\dot{V}$, der wie alle anderen Größen auch lokal und zeitlich variabel sein kann:

$$
\begin{aligned}
\dot{V}_{\text {Luft }} & =\frac{\dot{m}_{\text {Luft }}}{\rho_{\text {Luft }}}+\frac{\dot{m}_{\text {Luftfremd }}}{\rho_{\text {Luftfremd }}} \\
& \approx \frac{\dot{m}_{\text {Luft }}}{\rho_{\text {Luft }}} \text { mit } \frac{\rho_{\text {Luftfremd }}}{\rho_{\text {Luft }}} \ll 1
\end{aligned}
$$

Abbildung 1 zeigt einen offenen Rinderstall. Der Wind treibt Luft in und durch den Stall. Dabei 
werden sog. luftfremde Stoffe "nach draußen" befördert. Es geht darum, diesen Massenstrom zu quantifizieren. Luftvolumenstrom und Gaskonzentration sollen dabei getrennt gemessen werden.

Die Masse $m$ eines Stoffes, die Dichte $\rho$ des betreffenden Stoffes und das korrespondierende Volumen $V$ stehen in einem Zusammenhang. Bei zeitlich variierenden Luftvolumenströmen und konstanter Dichte (kein Einfluss von Temperatur- und Gasdruckänderungen) stellt der Massenstrom $\dot{m}$ das Produkt aus der Dichte $\rho$ und dem Luftvolumenstrom $\dot{V}$ dar (Gl. 5)

$$
\dot{m}=\frac{d}{d t} \int_{V} \rho \cdot d V
$$

Der Massenstrom eines Stoffes, der das System Stall über verschiedene Öffnungen verlässt, kann nur dann sinnvoll bestimmt werden, wenn der Luftvolumenstrom und die dazugehörige Gaskonzentration zeitgleich erfasst werden (Gl. 6):

$$
\dot{m}(t)=C(t) \cdot \dot{V}(t)
$$

Ein repräsentativer mittlerer Massenstrom, der z.B. für die Einordnung eines Stalles im Hinblick auf seine Umweltrelevanz von Bedeutung ist, führt auf die Produktmittelung von Konzentration und Volumenstrom:

$$
\dot{m}_{\text {Mittel }}=\overline{\dot{m}}=\overline{C(t) \cdot \dot{V}(t)} \neq \overline{C(t)} \cdot \overline{\dot{V}(t)}
$$

Das Ungleichheitszeichen in Gleichung 7 verschwindet, wenn einer der beiden Faktoren konstant ist. Bei Zwangslüftungen (siehe auch Abb. 3 oben) wird sicherlich, durch die tierphysiologischen Anforderungen bedingt, über einen längeren Zeitraum $\dot{V}(t)=$ konst $=\dot{V}$ gesetzt werden können, so dass dann $\dot{m}_{\text {Mittel }}=\overline{C(t)} \cdot \dot{V}$ gilt. In der freien Lüftung (Abb. 3 unten) schwanken beide Faktoren. Kennzeichnet $\varepsilon_{C}$ den Messfehler für $C(t)$ und $\varepsilon_{\dot{V}}$ den für $\dot{V}(t)$, dann folgt

$$
\begin{aligned}
\dot{m}= & \left(1+\varepsilon_{C}\right) \cdot C(t) \cdot\left(1+\varepsilon_{\dot{V}}\right) \cdot \dot{V}(t) \\
= & {\left[C(t)+\varepsilon_{C} \cdot C(t)\right] \cdot\left[\dot{V}(t)+\varepsilon_{\dot{V}} \cdot \dot{V}(t)\right] } \\
= & C(t) \cdot \dot{V}(t)+\varepsilon_{C} \cdot C(t) \cdot \dot{V}(t) \\
& +\varepsilon_{\dot{V}} \cdot C(t) \cdot \dot{V}(t)+\varepsilon_{C} \cdot \varepsilon_{\dot{V}} \cdot C(t) \cdot \dot{V}(t) \\
= & C(t) \cdot \dot{V}(t) \cdot\left(1+\varepsilon_{C}+\varepsilon_{\dot{V}}+\varepsilon_{C} \cdot \varepsilon_{\dot{V}}\right)
\end{aligned}
$$

$\varepsilon_{C}$ und $\varepsilon_{\dot{V}}$ in Gleichung 8 können positiv und/oder negativ sein, es gilt somit $0<\left|\varepsilon_{C}\right|$ bzw. $0<\left|\varepsilon_{\dot{V}}\right|$. 
Wenn ein mittlerer Massenstrom eines luftfremden Stoffes bestimmt werden soll, ist dann der Fehler, der durch die nicht zeitgleiche Mittelung des Produktes $C(t) \cdot \dot{V}(t)$ produziert wird, größer, als wenn die Faktoren getrennt gemittelt werden und dabei der Volumenstrom durch die pauschale Mittelung im Strömungsraum berechnet wird?

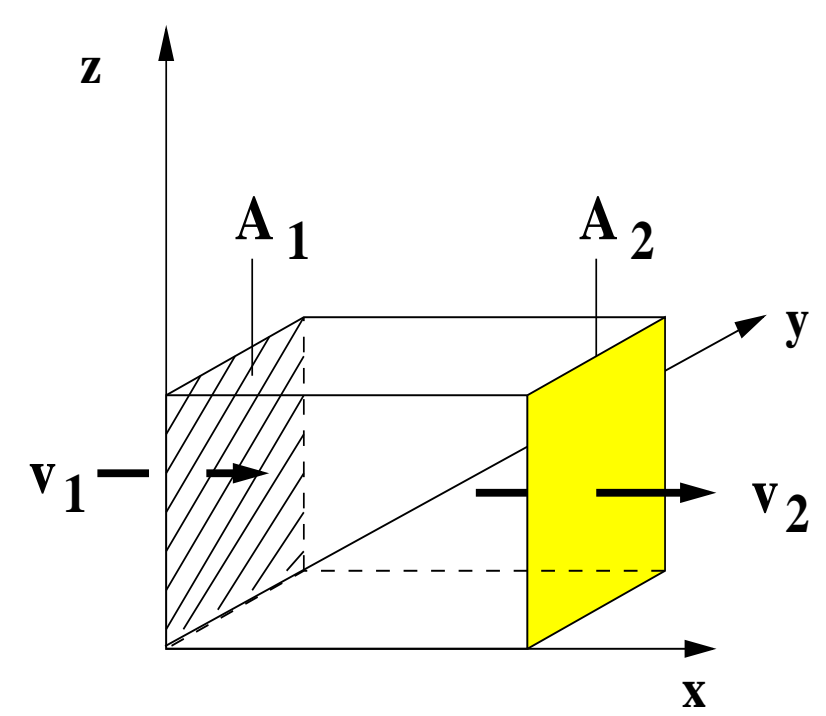

Abbildung 2: Betrachtung der zeitlichen Massenänderung zwischen zwei Flächen $A_{1}$ und $A_{2}$.

Der Übertritt stofflicher Emissionen aus dem definierten System in das nähere Umfeld hängt von der räumlichen Gestaltung des Stalles sowie der Größe und Lage seiner Zu- und Abluftöffnungen ab. Mit Blick auf Abbildung 2 gilt es, die zeitliche Änderung der Massenkonzentration eines Stoffes beim Übergang durch die Flächen $A_{1}$ und $A_{2}$ zu beschreiben. Betrachtet man beispielsweise ein offenes Stallgebäude für die Hähnchenmast (sog. Louisianastall) mit beidseitig geöffneten Seitenwandflächen (Abb. 3b), die über $100 \mathrm{~m}$ lang sein können und über $20 \mathrm{~m}$ entfernt voneinander liegen, so ist eine integrative Bestimmung des Volumenstromes nur bedingt - wenn überhaupt - möglich. Um frei gelüftete Systeme realistisch beurteilen zu können, sind Aussagen zum lokalen Strömungsgeschehen erforderlich. Es muss ein Übergang von der gesamtheitlichen (integralen) Betrachtungsweise zur lokalen (differentiellen) Betrachtungsweise geschaffen werden. Hierzu stehen skalare und vektorielle Größen zur Verfügung, um die Austauschvorgänge in einem System zu beschreiben. Dazu ist es erforderlich, sich intensiv mit dem Strömungsgeschehen in einem System auseinanderzusetzen. Praktische Beispiele, die das Gesagte verdeutlichen, sind in Abbildung 3 dargestellt.

Zwangsgelüftete Ställe stellen mit ihren Abluftschächten in erster Näherung Punktquellen dar. Sie weisen definierte, punktartige Kontaktflächen zur Umwelt auf. Eine Abhängigkeit des Emissionsmassenstromes von atmosphärischen Einflüssen der Außenluft ist in der Regel nicht gegeben. Die Ermittelung des Massenstroms aus Nutztierställen mit Zwangslüftung gestaltet sich daher mess- und rechentechnisch relativ einfach. 


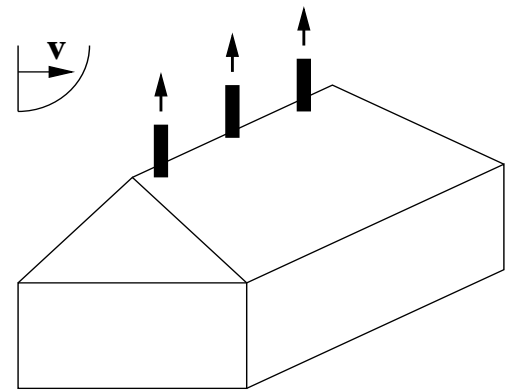

Zwangslüftungssystem mit Ausstoß über First

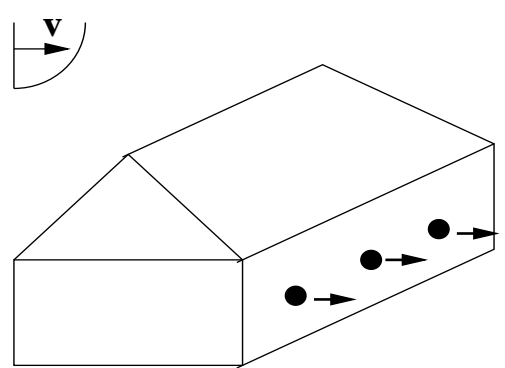

Zwangslüftungssystem mit seitlichem Ausstoß
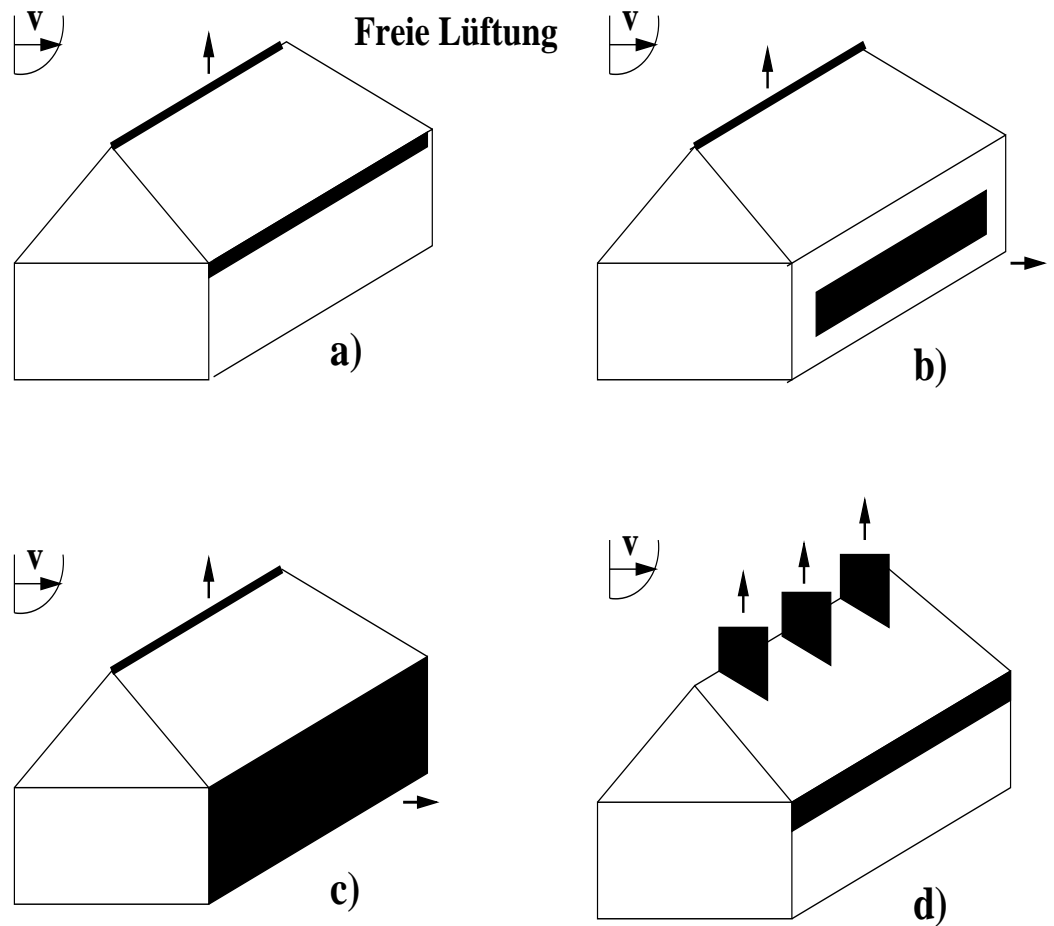

a) Traufen-First-Lüftung

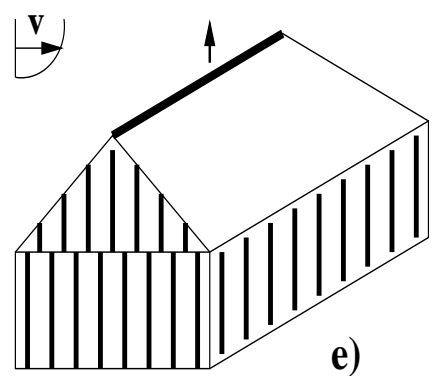

b) Offenstall mit vorherrschender Querlïftung

c) Offenfrontstall

d) Schwerkraftlüftung

e) Giebel- u. Seitenwandlüftung mit senkrechten Lüftungsschlitzen

Abbildung 3: Austrittsörter der Abluft bei zwangsgelüfteten Ställen (beide oberen Zeichnungen) bzw. Austauschflächen für windinduzierte Luftvolumenströme bei freigelüfteten Ställen (Zeichnungen a) bis e)). Die Ställe sind dem atmosphärischen Wind ausgesetzt. Das Piktogramm veranschaulicht das parabolische Windanströmprofil. 
Ein natürlich gelüftetes Stallgebäude stellt in der freien atmosphärischen Strömung aufgrund seiner Aufbauten ein Hindernis dar, welches eine Um- und Durchströmung erfährt. Hierdurch wird die stallinterne Strömung - damit ist die Strömung in dem durch die Stallwandungen eingeschlossenen Raum gemeint - beeinflusst, welche Rückwirkungen auf die Emissionsfreisetzungsvorgänge ausübt, die aber nicht Gegenstand der vorliegenden Arbeit sind. Frei gelüftete Ställe, in welcher Ausführungsform auch immer, sind bezüglich der Emissionen diffuse Quellen, vielfach Flächenquellen. Sie weisen schwer definierbare, teils großflächige Kontaktstellen zur Umwelt auf. Es besteht eine direkte Abhängigkeit von atmosphärischen Einflussgrößen, vor allem Windrichtung und Windgeschwindigkeit. Die Ermittelung des Massenaustrags aus Ställen mit natürlicher Lüftung gestaltet sich schwierig. Es besteht Handlungsbedarf, die realen Gegebenheiten bei der Durchströmung von frei gelüfteten Ställen wiederzugeben: es geht um die Quantifizierung der Luftvolumenströme.

Für die Bestimmung der gasförmigen Emissionsmassenströme von Ammoniak $\left(\mathrm{NH}_{3}\right)$, Methan $\left(\mathrm{CH}_{4}\right)$, Lachgas $\left(\mathrm{N}_{2} \mathrm{O}\right)$, Kohlendioxid $\left(\mathrm{CO}_{2}\right)$ und Wasserdampf $\left(\mathrm{H}_{2} \mathrm{O}\right)$ aus der Rinderhaltung werden zwei frei gelüftete Stalltypen ausgewählt: ein Stall mit Schwerkraftlüftung und ein Offenfrontstall. Neben der Erfassung der erforderlichen Gaskonzentrationen zur Beurteilung der Umweltrelevanz frei gelüfteter Rinderställe liegt der Schwerpunkt der Arbeit vorrangig in der Berechnung des Luftvolumenstromes. Dazu wird auf die Kompartimentalisierungsmethode zurückgegriffen, die bereits seit Jahrzehnten, vor allem im medizinischen Bereich, eingesetzt wird.

Für eine objektive Beurteilung der errechneten Luftvolumenströme werden zunächst unter Zuhilfenahme der numerischen Strömungssimulation (Referenzsystem) theoretische Vorversuche durchgeführt, um anschließend die Übertragbarkeit der Ergebnisse im praktischen Versuch einschätzen zu können. Die theoretischen Vorüberlegungen dienen ferner dem Ziel, die grundsätzlich vorhandenen Unterschiede in der Herangehensweise der verwendeten Kompartimentalisierungsmethode im Vergleich zu herkömmlichen Tracerverfahren offenzulegen. 


\section{Emissionsmassenstromermittelung mittels Tracern}

Grundlage der Tracertechnik ist die Verwendung von Tracern, sog. Spürgasen. Diese werden der eintretenden Außenluft oder der Luftströmung z.B. in einem Stall zugeführt, da sie im Allgemeinen ebenso wie die bereits im Stallraum vorhandenen Gasmoleküle transportiert und verdünnt werden. Tracerverfahren beruhen dabei auf Konzentrationsmessungen. Das bedeutet, dass bei einer bekannten Startkonzentration des Tracers dessen Verdünnung über einen bestimmten Zeitraum und an meist vorgegebenen Orten verfolgt wird. Durch die Übertragung der ermittelten Verdünnungswerte und damit der ermittelten Konzentrationsänderung des Tracers ist eine Quantifizierung des Luftvolumenstromes mit den unter 2.1.1 bis 2.1.3 beschriebenen Verfahren möglich.

\subsection{Luftvolumenstromermittelung bei homogener Tracerverteilung im Raum}

Ein probates Mittel zur Berechnung des Luftaustausches von Gebäuden besteht in der Freisetzung von Tracern im Gebäude. Die Art des verwendeten Tracers hängt von einer Vielzahl von Faktoren ab: Dauer des Versuches, Verfügbarkeit des Tracers, vorhandene Messtechnik etc.. Als Tracer werden beispielsweise $\mathrm{CO}_{2}, \mathrm{~N}_{2} \mathrm{O}, \mathrm{SF}_{6}$ oder Krypton 85 eingesetzt. Der letztgenannte Tracer gehört zu den radioaktiven Edelgasen, die zunehmend kritisch bewertet werden [70]. Dennoch weisen diese Tracer zusammen mit der erforderlichen Messtechnik elementare Vorzüge im Hinblick auf die zeitliche Auflösung der Messwerte (siehe dazu Kapitel 5.2) und damit auf die Aussagekraft von Luftvolumenstrombestimmungen auf.

Von der Vielzahl ausführlich dokumentierter Versuche in der Literatur soll stellvertretend auf NEDERHOFF ET AL. [50] und [34] verwiesen werden, die $\mathrm{N}_{2} \mathrm{O}$ bzw. $\mathrm{CO}_{2}$ als Tracergas einsetzten. Unabhängig vom verwendeten Tracer müssen gewisse Mindestanforderungen erfüllt werden ([59], [63], [73]), von denen die Wesentlichen aufgeführt werden:

- ungiftig für Mensch und Tier

- keine oder nur geringfügige Hintergrundkonzentration

- passives und lineares Verhalten, d.h. die Zugabe des Tracers in die Stallluft stellt einen rein additiven Vorgang dar; Wechselwirkungen von Tracer und Luft sind ausgeschlossen

- Transport des Tracers erfolgt wie der der Luft

- messtechnische Erfassbarkeit

- Wirtschaftlichkeit 
Das System, in welches ein Tracer eingebracht wird, wird als vollständig durchmischtes Volumen angesehen. Insbesondere bei Stallgebäuden mit einer hoch gelegenen Firstöffnung ist unter Umständen der Einfluss einer thermischen Schichtung auf die Tracerverteilung zu berücksichtigen. Die Erfassung der Tracerkonzentration kann durch Zählrohre, Ansaugschläuche, Sammler oder andere geeignete Probenahmevorrichtungen erfolgen, die im Weiteren vereinfachend unter dem Begriff Sensor zusammengefasst werden. Unter einem Sensor versteht man gemeinhin eine elektronische Funktionseinheit, die eine physikalische oder chemische Messgröße in ein elektrisches Signal transformiert [61]. Da im Rahmen der Untersuchungen elektronisch gesteuerte Zählrohre zum Einsatz kommen (Kapitel 5.2), ist der Begriff Sensor angebracht. Es handelt sich dabei nur um einen Messwertaufnehmer, dessen Messwerte zur Berechnung des Luftvolumenstromes verwendet werden. Messwertaufnehmer zur Erfassung der Gaskonzentration werden an den entsprechenden Stellen getrennt ausgewiesen.

Nachfolgend werden die drei gängigsten Tracermethoden zur Bestimmung des Volumenstromes vorgestellt.

\subsubsection{Abklingmethode}

Der Tracer wird einmalig und stoßartig in das System eingebracht. An verschiedenen Orten im Raum wird das Abklingen der Anfangskonzentrationsverteilung, in der Regel zeitversetzt, erfasst. In die Messwertewolke wird eine stetige Abklingfunktion gelegt, anhand derer eine mittlere Abklingkonstante ermittelt werden kann. Unter Kenntnis dieser Konstante sowie des Stallvolumens lässt sich der Volumenstrom auf indirekte Weise berechnen. Das zugrunde liegende Raumvolumen sollte nicht größer als $500 \mathrm{~m}^{3}$ sein [70].

Im Bilanzierungsraum bestimmt sich die Massenänderung zu Gleichung 9, wobei $F$ für einen Quellterm steht, der die Produktionsrate an Tracergas im Bilanzierungsraum kennzeichnet:

$$
\frac{d m}{d t}=\frac{d C_{\text {innen }}}{d t} \cdot V+\frac{d V}{d t} \cdot C_{i n n e n}+F
$$

Über die Bilanzierungsgrenze fließt die Masse (Gl. 10)

$$
\dot{m}_{\text {ein }}-\dot{m}_{\text {aus }}=C_{\text {außen }} \cdot \dot{V}_{\text {ein }}-C_{\text {innen }} \cdot \dot{V}_{\text {aus }}+F
$$

Bei starren Wandungen gilt Gleichung 11:

$$
\frac{d V}{d t}=0
$$

Da der Tracer einmalig in das Bilanzierungsraum eingebracht wird, ist $F=0$. Ferner folgt mit $C_{\text {außen }}=0$ und $C_{\text {innen }}=C$ 


$$
\begin{aligned}
V \cdot \frac{d C_{t}}{d t} & =-C_{t} \cdot \dot{V}_{\text {aus }} \\
C_{t} & =C_{0} \cdot e^{-\frac{\dot{V}_{\text {aus }}}{V} t}
\end{aligned}
$$

sowie $\alpha=\frac{\dot{V}_{\text {aus }}}{V}$ mit $\dot{V}_{\text {aus }}=$ konst.

$$
\left.\ln C\right|_{C_{0}} ^{C_{t}}=\int_{t_{t}}^{t_{0}} \alpha d t
$$

Der Term $\frac{\dot{V}_{a u s}}{V}$ im Exponent von Gleichung 13 charakterisiert den Luftvolumenstrom bezogen auf das Stallvolumen und kann mit dem Koeffizienten $\alpha$ beschrieben werden:

$$
\alpha=\frac{\ln \left(\widetilde{C_{0}}\right)-\ln \left(\widetilde{C_{t}}\right)}{t_{t}-t_{0}}
$$

$\alpha$ wird umgangssprachlich auch als (raum- und zeitbezogene) Luftwechselzahl bezeichnet, wobei $\alpha$ eine dimensionsbehaftete Größe $\left[\frac{1}{Z e i t}\right]$ ist. $\widetilde{C_{0}}=\frac{C_{0}}{C_{i}}$ steht für den Startkonzentrationsmesswert und $\widetilde{C_{t}}=\frac{C_{t}}{C_{i}}$ für den Endkonzentrationsmesswert zu einem beliebigen Zeitpunkt $t$ (jeweils an den Messpunkten) bezogen auf die Injektionskonzentration $C_{i}$ des Tracers im Stallraum. $t_{0}$ und $t_{t}$ stehen für die Zeitpunkte des Messanfangs bzw. Messendes. $\widetilde{C_{t}}$ und $\widetilde{C_{0}}$ sind als $l n$-Argumente dimensionslos.

Die Berechnung des Luftvolumenstromes mittels Abklingmethode erfolgt anhand von Gleichung 16

$$
\dot{V}_{\text {aus }}=\alpha \cdot V
$$

Die Abklingmethode liefert keine Auskunft über eine Luftverteilung im Bezugsraum [58]. Sie ist ein punktuelles Verfahren (Spotmessung), deren Ergebnis nur für die zur Messzeit vorherrschenden Randbedingungen repräsentativ ist. Die größten Probleme in der Genauigkeit dieses Messverfahrens liegen in der Annahme einer homogenen Traceranfangsverteilung im System sowie in der ungenauen Positionierung der Probenahmepunkte. Bei stark inhomogener Tracerverteilung im System und zeitlich instabilen Strömungsverhältnissen ist die Berechnung des Luftvolumenstromes mittels Abklingmethode "reiner Zufall" [24].

Der Vorteil der Abklingmethode liegt in der zeitextensiven Anwendung des Verfahrens, es wird hierbei nur wenig Tracer benötigt. Das Abklingverfahren kann gezielt eingesetzt werden, etwa um den Luftwechsel bei geöffneten Toren, bei unterschiedlichen Jalousiestellungen etc. zu bestimmen, also bei Versuchen, die kurzzeitig durchgeführt werden. Bestimmte Lüftungsformen, z.B. die TraufenFirst-Lüftung, erleichtern die Anwendung des Verfahrens, da in etwa definierte Strömungsbedingungen vorliegen. Die sich stallintern stetig ändernden Strömungsfelder - bedingt vor allem durch Wind- 
richtungsänderungen - können aber auch in diesen Fällen zu einer Fehlinterpretation des errechneten Volumenstromes führen (siehe auch Abschnitt 2.1.4). Da mittels Abklingmethode keinerlei Aussagen zum Strömungsverlauf möglich sind, ist eine sorgfältige Prüfung der Ergebnisse vor allem bei wechselnden Windanströmrichtungen erforderlich.

\subsubsection{Methode der konstanten Tracerinjektion}

Bei der Methode der konstanten Tracerinjektion, auch als Methode der konstanten Emission bezeichnet, wird der Tracer mit einer bekannten, fixen Konzentration, gleichbleibend über den gesamten Versuchszeitraum, in das System injiziert. An den Sensorpunkten, welche als wahrscheinliche Austrittorte der Abluft angenommen werden, wird das Gas angesaugt. Infolge eines unterschiedlich starken Luftdurchsatzes variiert die dort erfasste Tracerkonzentration.

Da der Tracer in diesem Fall nicht einmalig sondern kontinuierlich zugeführt wird, muss der Tracerquellterm $F$ berücksichtigt werden [27]. Ausgehend von Gleichung 10 ergibt sich nun mit $F=$ konst. (aber $F \neq 0$ ) sowie $C_{\text {außen }}=0$ und $C_{\text {innen }}=C$ die folgende Ausgangsgleichung 17

$$
V \cdot \frac{d C_{t}}{d t}=-C_{t} \cdot \dot{V}_{a u s}+F
$$

Für die Tracerkonzentration zu einem beliebigen Zeitpunkt $t$ gilt Gleichung 18

$$
C_{t}=\frac{F}{\dot{V}_{\text {aus }}}+\left(C_{0}-\frac{F}{\dot{V}_{\text {aus }}}\right) \cdot e^{-\frac{\dot{V}_{a u s}}{V} t}
$$

Da zu Beginn der Messung kein Tracer im Bilanzierungsraum vorhanden ist, folgt mit $C_{0}=0$ sowie unter Annahme von $\dot{V}_{\text {aus }}=$ konst. Gleichung 19

$$
C_{t}=\frac{F}{\dot{V}_{\text {aus }}} \cdot\left(1-e^{-\frac{\dot{V}_{\text {aus }}}{V} t}\right)
$$

Bei etwa konstanten Luftwechselzahlen $\alpha$ wird nach einer endlichen Zeit ein Konzentrationsgleichgewicht des Tracers erreicht. Die Berechnung des Luftvolumenstromes mittels Methode der konstanten Tracerinjektion kann dann anhand von Gleichung 20 erfolgen

$$
\dot{V}_{\text {aus }}=\frac{F}{C_{t}}
$$

Bei stark schwankenden Luftwechseln sollte die Methode der konstanten Tracerinjektion nicht eingesetzt werden [58], da die Injektionskonzentration in der Regel zeitversetzt an den Sensoren registriert 
wird. Die konstante Tracerinjektion eignet sich für Langzeitmessungen bis hin zu mehreren Monaten, wenn die vorherige Einschränkung hinsichtlich stark variierender Luftwechselzahlen beachtet wird. Eventuelle tageszeitliche Konzentrationsschankungen und -maxima werden aber aufgrund der vorhandenen Messzeitintervalle und der zeitlich verzögerten Reaktion des Messgerätes auf Windgeschwindigkeitsänderungen (time delay) selten erfasst [2].

Nachteilig wirkt sich der relativ hohe Tracerverbrauch aus. Sinnvoll sind daher Messgeräte, die die Tracerkonzentration im parts per billion (ppb: $10^{-6}$ ) oder im parts per trillion (ppt: $10^{-9}$ ) Bereich exakt detektieren können.

\subsubsection{Methode der variablen Tracerinjektion}

Der Tracer wird an vorgegebenen Injektionsorten in den Stallraum eingebracht. Das Ziel der Methode der variablen Tracerinjektion, auch als konstante Konzentrationsmethode geläufig, besteht in der Konstanz der Tracerkonzentration an den Sensoren. Dies wird durch eine Regelung des Tracergasstromes an den Injektionspunkten erreicht, d.h. $F=F(t)$.

Ausgehend wiederum von Gleichung 10 ergibt sich mit $C_{a u ß e n}=0$ und $C_{\text {innen }}=C$ die folgende Gleichung 21

$$
V \cdot \frac{d C_{t}}{d t}=-C_{t} \cdot \dot{V}_{a u s}+F_{t}
$$

Da zu Beginn der Messung kein Tracer im Bilanzierungsraum vorhanden ist, folgt $C_{0}=0$. Wie bereits oben erwähnt, wird bei dieser Methode versucht, die (Ziel-)Tracerkonzentration an den Sensoren $\left(C_{Z i e l}=C_{t}\right)$ durch eine entsprechende Steuerung der Tracergasmenge annähernd konstant zu halten. Die Berechnung des Luftvolumenstromes mittels Methode der variablen Tracerinjektion erfolgt dann anhand von Gleichung 22

$$
\dot{V}_{\text {aus }}=\frac{F_{t}}{C_{\text {Ziel }}}
$$

Aufgrund des Bedarfs an hochautomatisierter Technik zur Regelung des Tracergasinjektionsstromes beschränkt sich der Einsatz dieses Verfahrens bislang nur auf den Bereich der Forschung und Entwicklung. Nachteilig kann sich auch bei diesem Verfahren, in Abhängigkeit von der Höhe des Luftwechsels, der hohe Tracerbedarf auswirken.

Die Genauigkeit der drei vorgestellten Verfahren hängt neben der Positionierung der Messpunkte im Wesentlichen von der Erzielung einer ausreichenden Vermischung des Tracers mit der Stallluft ab. Die Annahme einer homogenen Vermischung ist in der Praxis jedoch unrealistisch [4]. Fehlersenkend 
wirken ein mehrpunktuelles Einbringen des Tracers in den Stallraum [40] sowie die sachgerechte Wahl der Probenahmepunkte. Dennoch ist es gerade bei Anwendung der Methode der konstanten und variablen Tracerinjektion sinnvoll, wenn nicht sogar zwingend erforderlich, Volumenstrommessungen nur bei ausgewählten Wetterlagen durchzuführen.

\subsubsection{Unzulänglichkeiten bei der Mittelwertbildung und Sensorpositionierung}

Die Kenntnis der Luftwechselzahl $\alpha$ in landwirtschaftlichen Nutztierställen ermöglicht es, das Lüftungssystem so auszulegen, dass wichtige Klimaparameter wie beispielsweise Temperatur, Luftfeuchtigkeit, Stoffkonzentration etc. innerhalb vorgegebener Grenzwerte eingehalten werden können. Neben einer möglichen Überschreitung von Grenzwerten interessiert unter dem Aspekt des Umweltschutzes auch, was im Mittel aus einem System emittiert wird. Im Mittel heißt, dass zeitlich gemittelte Massenströme bestimmt werden müssen. Massenströme verlassen im Bereich der Tierhaltung über die Schnittstelle zur Umwelt den Stall. Wenn diese Schnittstellen variieren, dann ist auch die lokale Mittelung neben der zeitlichen zu berücksichtigen. Das gilt insbesondere für die freie Lüftung. Die vorhandenen Unzulänglichkeiten von Tracerverfahren bzw. Berechnungsmethoden bei der Mittelwertbildung und Sensorpositionierung sollen anhand konkreter Beispiele aufgezeigt werden.

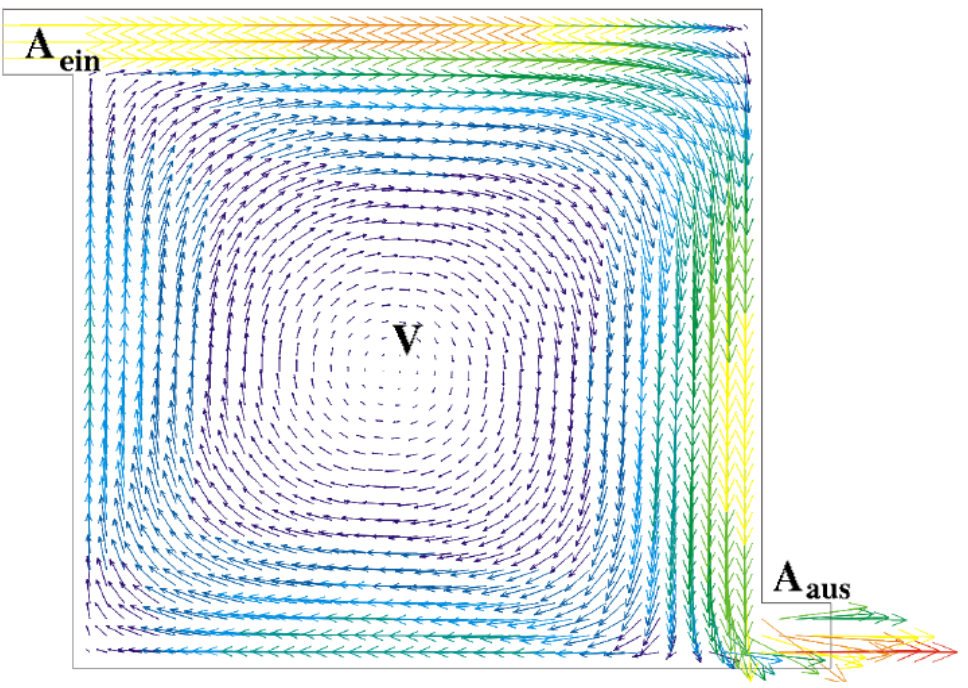

Abbildung 4: Geschwindigkeitsfeld bei der Durchströmung eines zweidimensionalen Raumquerschnittes ( $2 \mathrm{~m} \times 2 \mathrm{~m}$ ). Die Pfeile der Geschwindigkeitsvektoren geben die Strömungsrichtung an. Rot und gelb markierte Vektoren charakterisieren hohe, violette und blaue Vektoren geringe Strömungsgeschwindigkeiten; $R e=13.000$ bei einer Lufttemperatur von $20^{\circ} \mathrm{C}$.

Abbildung 4 zeigt einen zweidimensionalen Raum mit einer Höhe und einer Breite von jeweils zwei Metern. Die Luft wird oben links direkt unter der Raumbegrenzung mit einer konstanten Geschwin- 
digkeit hineingedrückt und strömt unten rechts frei hinaus. Die Hauptströmung fließt aufgrund des links oben eintretenden Impulsstrahls mit hohen Geschwindigkeiten entlang der oberen und rechten Seiteninnenwand direkt zum Auslass. Sie ist ein Teil des internen Wirbels, welcher in etwas abgeschwächter Form an der unteren und linken Seiteninnenwand zurück zum Einlass strömt. In der Mitte des Raumes bildet sich ein Bereich geringer Geschwindigkeit aus. Die Simulation dieses Beispiels erfolgte bei einer Reynoldszahl Re (Verhältnis von Trägheits- und Reibungskräften) von etwa 13.000. Damit handelt es sich um eine turbulente Strömung (siehe Abb. 14). In landwirtschaftlichen Nutztierställen ist mit $R e$-Zahlen von etwa 7.000 bis $8.000 \mathrm{zu}$ rechnen, diese liegen in der Größenordnung von Konferenzsälen [29] und sind ebenfalls turbulent.

Das Raumvolumen $V$ sowie die Eintritts- und Austrittsöffnung $\left(A_{\text {ein }}, A_{\text {aus }}\right)$ sind konstant. Mit Hilfe der numerischen Strömungssimulation (Kapitel 3.3) wird der Raum in 1.600 Raumelemente zerlegt. Nach Beendigung der Simulationsrechnung sind die Strömungsgeschwindigkeit und -richtung für jedes dieser Raumelemente bekannt. Somit kann das reale örtliche Strömungsgeschehen exakt wiedergegeben werden.

Im Vergleich zur experimentellen Vorgehensweise bei Feldmessungen muss dabei ein essentieller Aspekt berücksichtigt werden, der im Nachfolgenden erläutert wird.

\section{Numerik versus Messtechnik}

Mit Hilfe der numerischen Strömungssimulation (siehe Kapitel 3.3) wird der Raum in endlich kleine Raumelemente bzw. Raumpunkte zerlegt. Jeder Raumpunkt kann dabei theoretisch als Sensor zur Aufzeichnung der Tracerkonzentration angesehen werden. Eine Erhöhung der Gitterpunkte führt zu einer stabileren Lösung und damit zu genaueren Volumenströmen. Der Tracer wird der Luftströmung zugegeben, eine homogene Vermischung des Tracers mit der Luft ist gegeben. Nach erfolgter Simulationsrechnung (ohne Zeitschritte!) kann das Strömungsfeld an endlich vielen Raumpunkten (1.600 im Beispiel) charakterisiert werden. An jedem dieser Punkte ist der Geschwindigkeits- bzw. Konzentrationswert bekannt. Im Gegensatz zur Numerik kann der Untersuchungsraum, aufgrund der in der Praxis beschränkten Anzahl an Sensoren, weniger gut aufgelöst werden. Unabhängig davon macht es keinen Sinn, den Stall vollständig mit Sensoren zu bestücken, da dann kein Luftaustausch stattfinden würde. In der Messtechnik werden Zeitreihen gefahren, anhand derer eine inverse Lösung herbeigeführt wird. Zusätzliche Aussagen über definierte Geschwindigkeiten an Ein- und Auslass etc. können, speziell im Falle einer natürlichen Lüftung, nicht getroffen werden.

Die gefärbten Flächen in Abbildung 5 stehen jeweils für eine betrachtete Übergangsfläche für den möglichen Massenaustausch. In der Numerik kann der betreffende Messpunkt direkt als "Austauschfläche" an diesem Punkt angesehen werden. In der Messtechnik fungiert ein Messpunkt als Repräsentant für eine weitaus größere Fläche, innerhalb der derselbe Messwert an jeder Stelle angenommen 


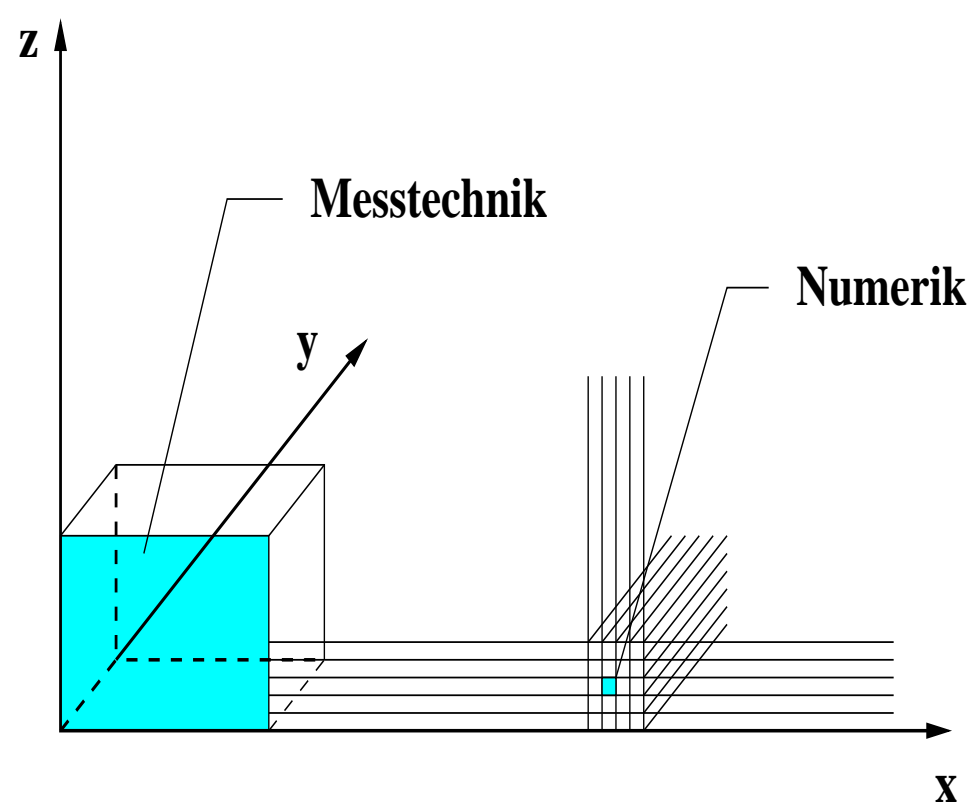

Abbildung 5: Unterschied zwischen Numerik und Messtechnik. Der Massenaustausch findet über die gefärbten Flächen statt. In der Numerik kann der Punkt der Probenahme gleichzeitig auch als Austauschfläche angenommen werden. In der Messtechnik ist der Punkt der Probenahme als "Stichprobe" anzusehen, die für eine weitaus größere Austauschfläche als repräsentativ angenommen werden muss.

wird. Nur für einige wenige Gitterpunkte stehen Konzentrationsmessreihen zur Verfügung (diese sind in Tab. 9 aufgelistet).

In Feldmessungen ist die Annahme einer inhomogenen Vermischung des Tracers mit dem Trägermedium realistisch. Dieser Sachverhalt wird in Kapitel 4.4 behandelt. Somit existieren im betrachteten System Bereiche/Felder unterschiedlicher Tracerkonzentrationen. Über die Größe, die Abgrenzung oder den Verlauf dieser Konzentrationsbereiche können keine Angaben gemacht werden. Sie könnten beispielsweise wie in der oberen Zeichnung von Abbildung 20 ausgebildet sein.

Der Strömungsverlauf ist a priori unbekannt, deshalb sind keine Aussagen über einen sicheren Ort der Sensorpositionierung möglich, vor allem nicht bei großen Ein- und Austrittsflächen. Es stellt sich die Frage, inwieweit es möglich ist, mit beschränkter Messtechnik die relevanten Punkte im Raum zu besetzen bzw. welche Anzahl an Sensoren für die Bestückung des Raums in Abbildung 4 benötigt wird. Dazu werden Vorüberlegungen von [24] aufgegriffen.

In Abbildung 6 strömt Frischluft in einen zweidimensionalen Raum von 1,3 m Länge und 0,7 m Breite in der linken unteren Seitenwand ein. Die Austrittsöffnung befindet sich oben auf der gegenüberliegenden Seitenwand. An den Punkten A, B und C erfolgt ein zeitlich begrenztes Einbringen des Tracers. Es werden 13 Sensoren an beliebigen Punkten im Raum positioniert. Die errechneten 


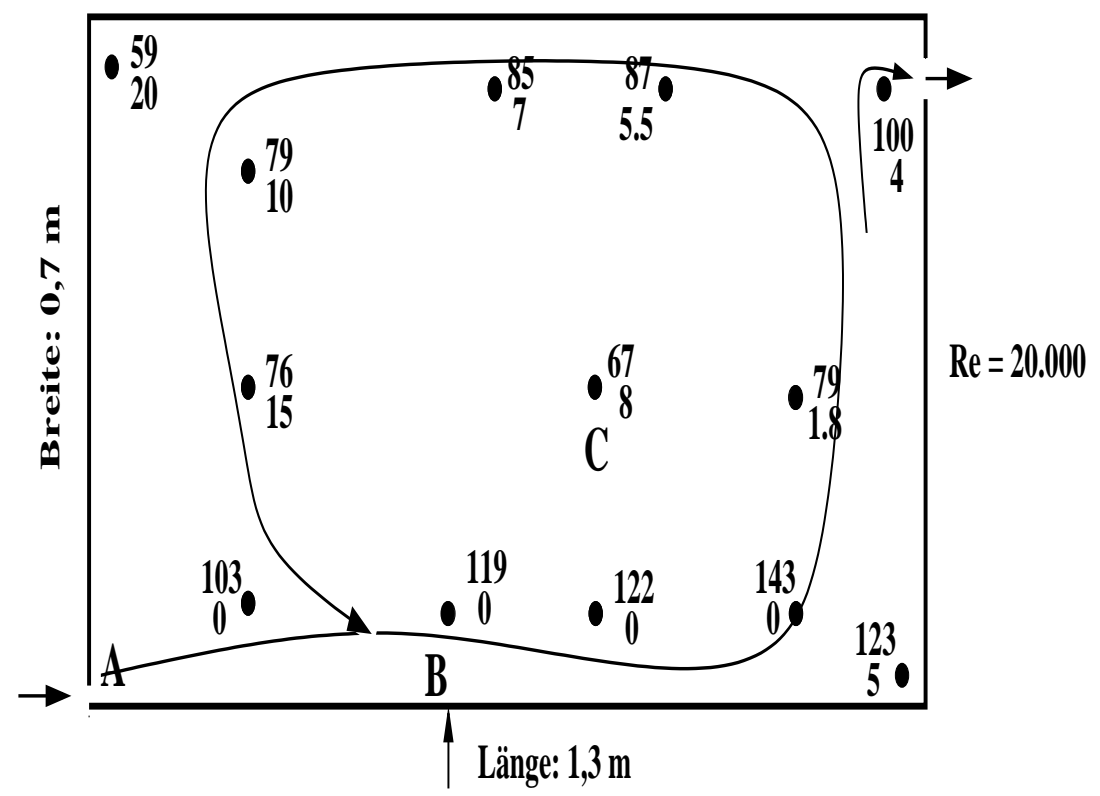

Abbildung 6: Ansprechzeit der Tracerkonzentration (untere Zahl, [Zeit*100]) und Werte der Luftwechselkoeffizienten (obere Zahl, [1/Zeit *100]) an verschiedenen Raumpunkten. Zusätzlich zur Originalliteratur ist der Strömungsverlauf vereinfacht dargestellt. Der Einlass befindet sich links unten, die Austrittsöffnung recht oben. An den Punkten A, B und C erfolgt die Tracerdosierung.

Luftwechselkoeffizienten sowie die Ansprechzeiten der Tracerkonzentration sind eingezeichnet. Die Ansprechzeit ist die Zeitspanne, die vergeht, bis die Tracerkonzentration erstmals an einem Punkt erfasst wird. In gleicher Weise kann die Ansprechzeit auch auf das gezeigte Beispiel in Abbildung 4 übertragen werden: sie gibt in diesem Fall die Zeit an, die vergeht, bis die eingebrachte Frischluft erstmals zu einem Abklingen der Tracerkonzentration an einem Sensor führt.

Wie in Abbildung 6 zu erkennen ist, bewirkt das vorhandene Strömungsfeld eine zeitlich versetzte Registrierung der Tracerkonzentrationen an den Sensoren in der oberen linken Bildhälfte. Das alleinige Heranziehen dieser Positionen zur Volumenstrombestimmung führt zu einer Unterschätzung des tatsächlichen Luftaustausches. Strenggenommen muss jeder Sensor zur Volumenstrombestimmung herangezogen werden, da aufgrund der Unkenntnis des Strömungsfeldes jeder Messwert der theoretisch Richtige sein kann. Es ist somit purer Zufall, wenn das Strömungsfeld durch die vorgenommene Messpunktanordnung und Tracerdosierung richtig gedeutet wird [13],[24].

Für das gewählte Simulationsbeispiel in Abbildung 4 wird als Besatz zunächst ein Sensor im Zentrum positioniert. Nachfolgend wird die Sensorzahl auf 9, 25 und 100 (Abbildung 7) symmetrisch erhöht. In Abbildung 8 sind die Ergebnisse in Form der Luftwechselkoeffizienten eingetragen. 

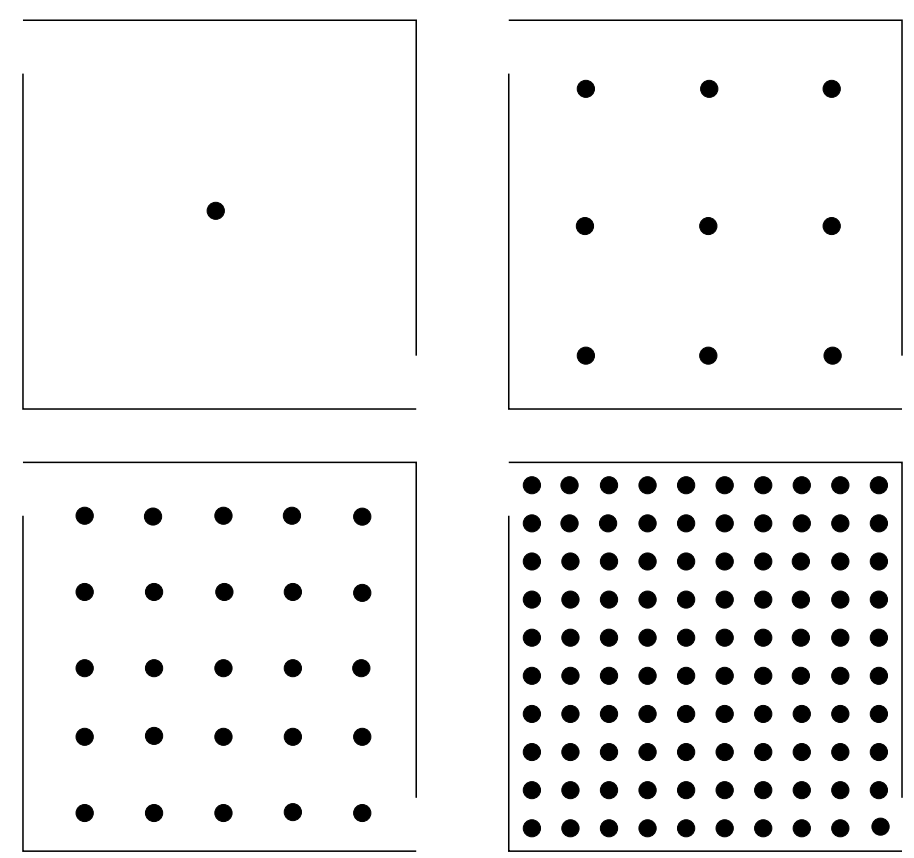

Abbildung 7: Bestückung eines zweidimensionalen Raumquerschnittes ( $2 \mathrm{~m}$ x $2 \mathrm{~m}$ ) mit 1, 9, 25 und 100 Sensoren.
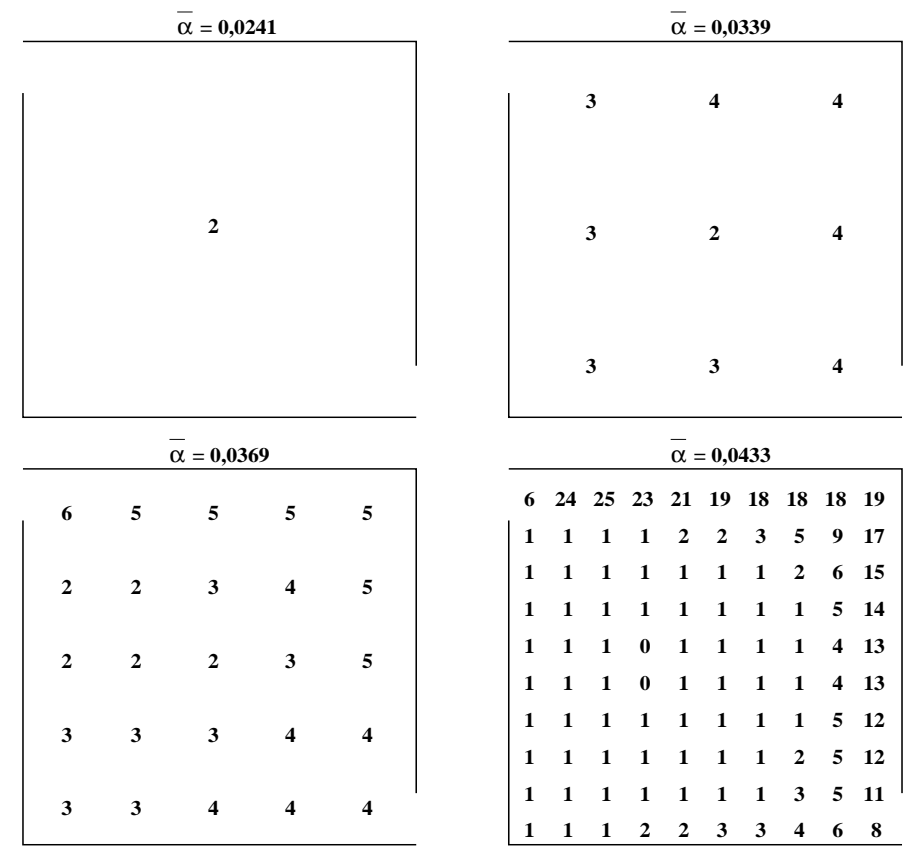

Abbildung 8: Höhe der Luftwechselkoeffizienten $\left(* 10^{2}\right)$ in einem zweidimensionalen Raumquerschnitt ( $2 \mathrm{~m}$ x $2 \mathrm{~m}$ ) bei einem Besatz von 1, 9, 25 und 100 Sensoren. 

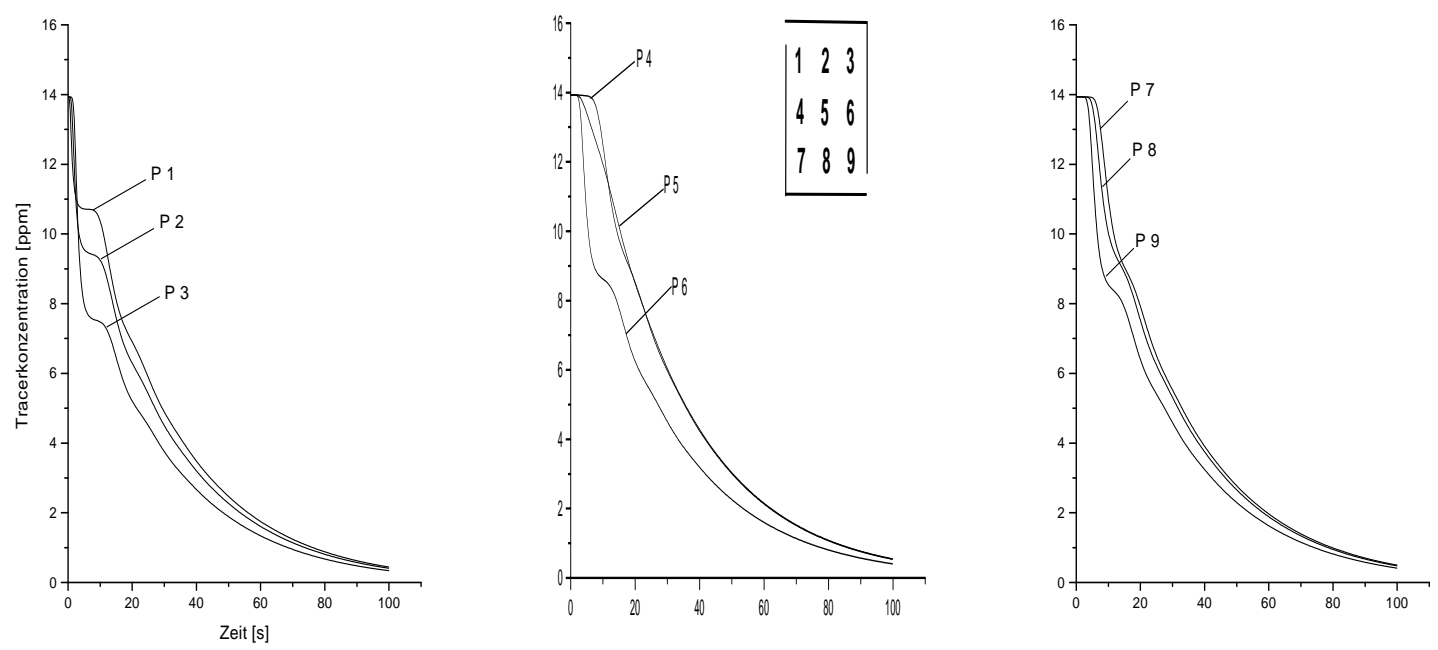

Abbildung 9: Abklingkurven der Tracerkonzentration [ppm] an neun Messpunkten $\left(\mathrm{P}_{1}\right.$ bis $\left.\mathrm{P}_{9}\right)$ im Zeitraum von $100 \mathrm{~s}$ bei der Durchströmung eines zweidimensionalen Raumquerschnittes $(2 \mathrm{~m} \times 2 \mathrm{~m})$ mit $v=1 \mathrm{~m} / \mathrm{s}$ am Einlass.

Führt nun die Erhöhung der Sensorzahl zu einer Erhöhung der Sicherheit in der Volumenstrombestimmung? Um diese Frage zu beantworten, muss zusätzlich die Vorgehensweise bei der Mittelwertbildung betrachtet werden. Dazu sei das Beispiel bei einem Besatz mit 9 Sensoren herausgegriffen. Die Abklingkurven an den 9 Messorten sind in Abbildung 9 ersichtlich. Die zeitlich versetzten Ansprechzeiten sind zu erkennen. Da kein Sensor als allgemeingültig für das Strömungsgeschehen angesehen werden kann, wird eine mittlere Abklingkonstante für jeden Sensor berechnet. Neben der Schwierigkeit der Vorhersage der relevanten Sensorpositionierung kommt ein weiteres Problem hinzu: Welcher Kurvenabschnitt ist repräsentativ für die Berechnung der Luftwechselkoeffizienten? Stellvertretend werden die Abklingkurven an den Messpunkten $\mathrm{P}_{3}, \mathrm{P}_{5}$ und $\mathrm{P}_{7}$ betrachtet. Tabelle 1 gibt die aus den Simulationsdaten ermittelten Luftwechselkoeffizienten für die in Abbildung 10 gekennzeichneten Abschnitte an.

Wie Tabelle 1 ausweist, besteht die Unzulänglichkeit bei der Mittelwertbildung darin, dass a priori kein Kurvenabschnitt als allgemein gültig herausgegriffen werden kann.

In der Praxis ist es üblich, den Abschnitt jeder Abklingfunktion zu betrachten, der ein stetiges Absinken der Tracerkonzentration verzeichnet. Das Vorhandensein turbulenter Strömungen in Ställen (Kapitel 3.2.2) hat zur Folge, dass sich die Abschnitte stetigen Abfalls der jeweiligen Abklingfunktionen nicht überlagern, sondern zeitversetzt auftreten. Noch gravierender macht sich dieser Sachverhalt bei Messtechniken bemerkbar, die z.B. aufgrund von erforderlichen Wartezeiten für die Spülung der Probenahmeschläuche keine zeitgleiche Messwerteerfassung vornehmen können. 


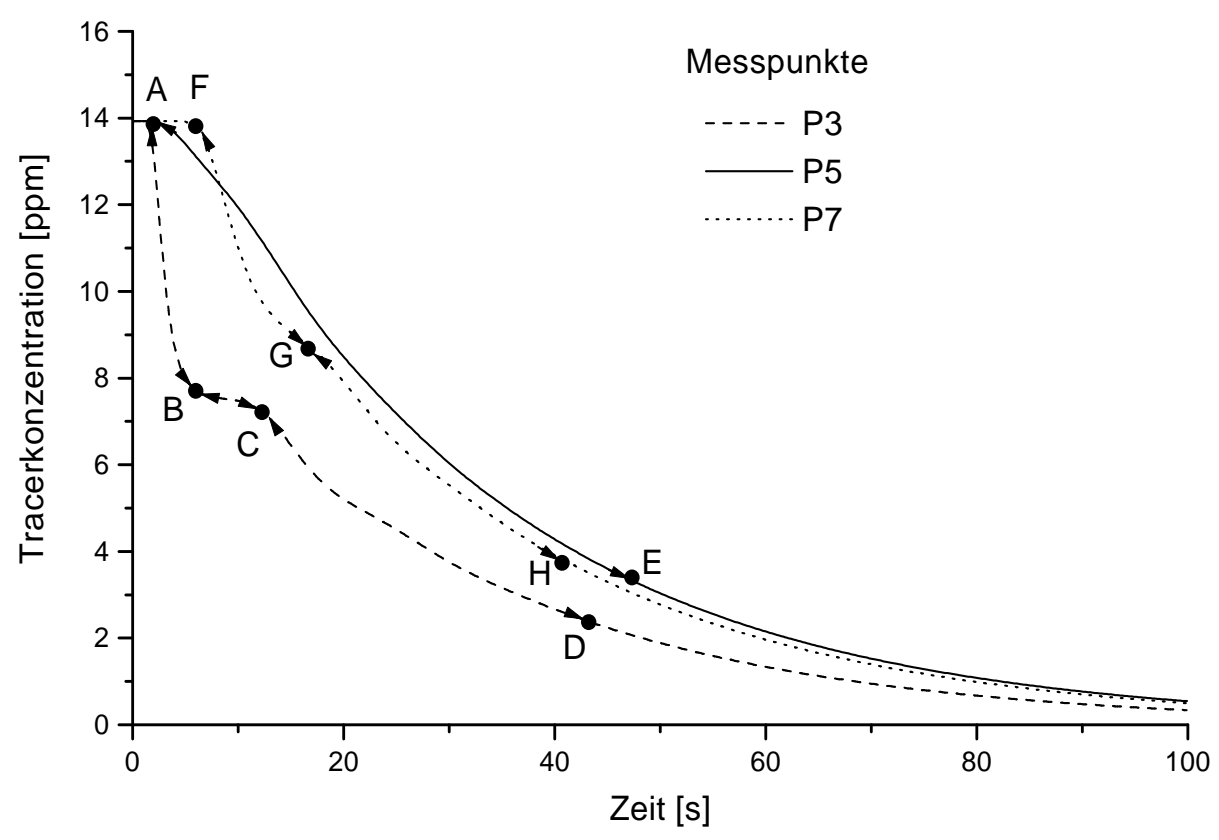

Abbildung 10: Verlauf der Abklingkurven an den Messpunkten $\mathrm{P}_{3}, \mathrm{P}_{5}$ und $\mathrm{P}_{7}$ bei der Durchströmung eines zweidimensionalen Raumquerschnittes (2m x $2 \mathrm{~m})$ mit $v=1 \mathrm{~m} / \mathrm{s}$ mit eingezeichneten Abschnitten zur Bestimmung der Luftwechselkoeffizienten $\alpha$.

Tabelle 1: Luftwechselkoeffizienten $\alpha$ für verschiedene Kurvenabschnitte von drei ausgewählten Abklingkurven an den Messpunkten $\mathrm{P}_{3}, \mathrm{P}_{5}$ und $\mathrm{P}_{7}$ bei der Durchströmung eines zweidimensionalen Raumquerschnittes $(2 \mathrm{~m} \mathrm{x} 2 \mathrm{~m}) \mathrm{mit} v=1 \mathrm{~m} / \mathrm{s}$.

\begin{tabular}{|c|c|c|c|}
\hline Abschnitt & Sensor 3 & Sensor 5 & Sensor 7 \\
\hline \hline A - B & 0,1300 & - & - \\
\hline B - C & 0,0131 & - & - \\
\hline C - D & 0,0359 & - & - \\
\hline A - E & - & 0,0316 & - \\
\hline F - G & - & - & 0,0444 \\
\hline G - H & - & - & 0,0341 \\
\hline
\end{tabular}


Überträgt man die o.g. Vorgehensweise (lokale Mittelung) auf das Problem der zeitversetzten Messwerteerfassung, so werden mittlere Abklingkonstanten zu unterschiedlichen Zeitpunkten bestimmt. Zwangsläufig ist damit nur die Berechnung eines mittleren Volumenstromes - der sich aus der Summe der mittleren Luftwechselkoeffizienten aller relevanten Kurvenabschnitte einer Abklingfunktion zusammensetzt - für den gesamten Zeitraum möglich.

Für die in Abbildung 7 gezeigten Beispiele lauten die gemittelten Luftwechselkoeffizienten und damit der daraus resultierende mittlere Volumenstrom (wobei die Raumtiefe als $\infty$ groß gegenüber der Breite und der Höhe des Raumes angenommen wird) für den gesamten Untersuchungsraum und Untersuchungszeitraum wie folgt:

1 Sensor: $0,0241 / \mathrm{s} b z w \cdot 0,0963 \mathrm{~m}^{3} / \mathrm{s}$

9 Sensoren: $0,0339 / \mathrm{s}$ bzw. $0,1354 \mathrm{~m}^{3} / \mathrm{s}$

25 Sensoren: 0, 0369/s bzw. 0, $1476 \mathrm{~m}^{3} / \mathrm{s}$

100 Sensoren: $0,0433 / \mathrm{s} b z w .0,1734 \mathrm{~m}^{3} / \mathrm{s}$

Die exakten Werte für die Abklingkonstante bzw. für den Volumenstrom im gewählten zweidimensionalen Raum betragen stets 0,0500/s bzw. 0, $2000 \mathrm{~m}^{3} / \mathrm{s}$. Fazit: Um in einem $2 \mathrm{~m}$ x $2 \mathrm{~m}$-Raum den Volumenstrom nach Abklingmethode mit etwa 13\%-iger Genauigkeit zu bestimmen, sind mindestens 100 Sensoren erforderlich. In der Praxis sind 80 Sensoren (bei zeitgleicher Messwerterfassung!) das momentane Maximum. Zudem liegen praxisübliche Rinderställe in einer Größenordnung von durchschnittlich $20 \mathrm{~m}$ x $50 \mathrm{~m}$ bis hin zu $100 \mathrm{~m}$ x $100 \mathrm{~m}$. Der eigentliche Vorteil, der bereits durch die Erhöhung der Sensorzahl im Strömungsgebiet von 1 auf 9 entsteht, verpufft durch die erforderliche Berechnung einer mittleren Abklingkonstante am jeweiligen Sensor. Möglichkeiten, diesen Vorteil dennoch nutzbar zu machen, bestehen in der Verwendung des Kompartimentalisierungsverfahrens, welches die Grundlage dieser Arbeit darstellt und ausführlich in Kapitel 4 behandelt wird.

Bei Traufen-First-Lüftungen (Abbildung 3a)) kann es zu gerichteten Luftströmen kommen. Durch die Überströmung des Gebäudes quer zum Stall entsteht ein Unterdruck am First, der, neben dem stallinternen thermischen Auftrieb, ein Aufsteigen der Luftmassen in Richtung First bewirkt. Die Ausführung beeinflusst dabei die Luftaustauschvorgänge ([23],[6]). Durch das Einbringen von Tracern in die traufseitigen Öffnungen und das Ansaugen der Abluft am First (Ringleitsystem) kann die Berechnung des Luftaustausches ebenfalls mittels Abklingmethode erfolgen [12],[66], wobei die aufgezeigten Unzulänglichkeiten bei der Mittelwertbildung auch für diese Messkonfiguration gelten. Es werden hierbei nur die Schnittstellen des Systems zur Umgebung betrachtet. 

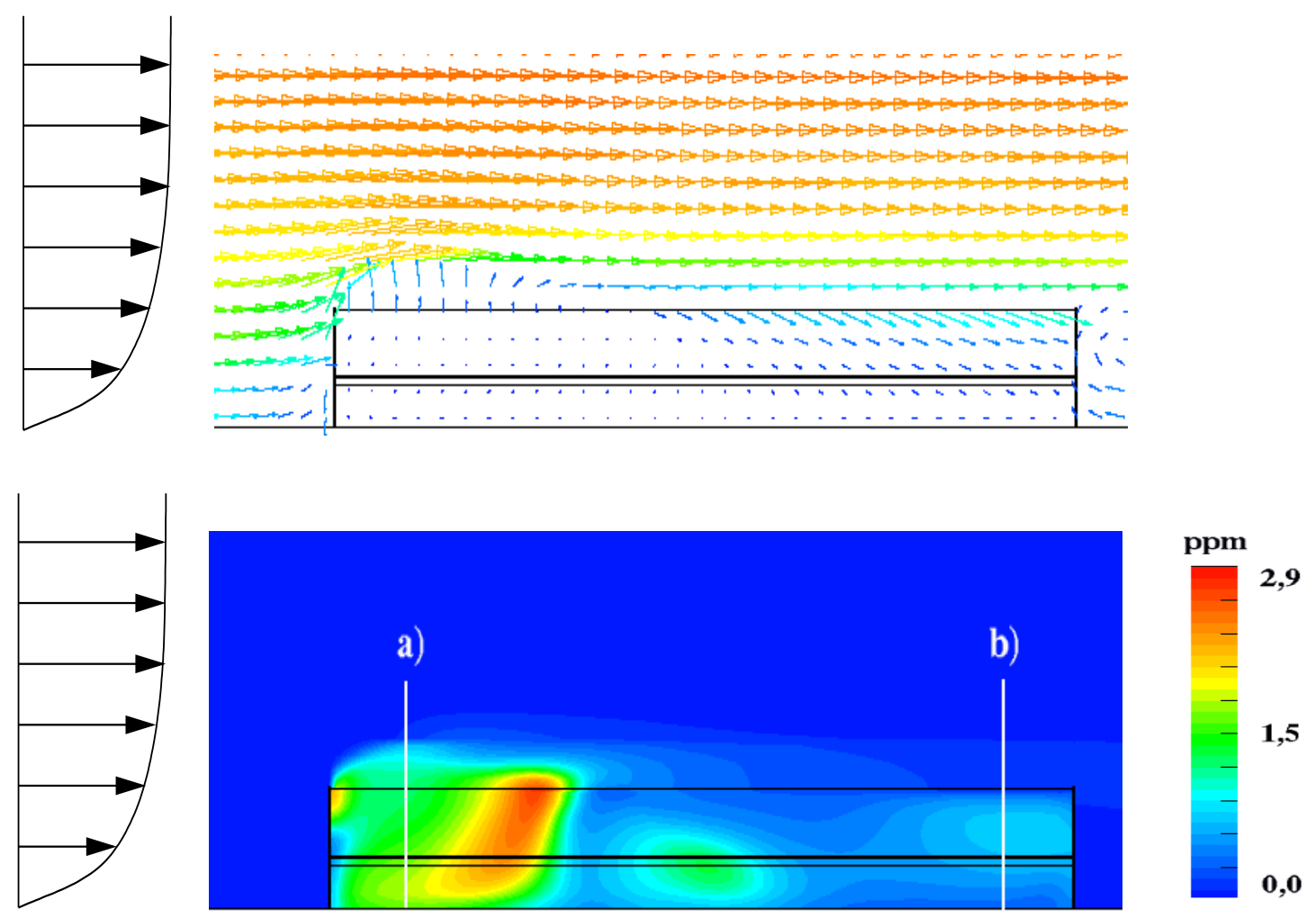

Abbildung 11: Vertikaler, firstmittiger Querschnitt des Geschwindigkeitsprofils (oben) und Konzentrationsprofils (unten) in einem Traufen-First-Stall bei einer firstparallelen Anströmung von links. Bereiche geringer Geschwindigkeiten bzw. Konzentrationen sind blau, Bereiche hoher Geschwindigkeiten bzw. Konzentrationen sind rot eingezeichnet. Die Windgeschwindigkeit des parabolischen Anströmprofils in $10 \mathrm{~m}$ Höhe beträgt jeweils $3 \mathrm{~m} / \mathrm{s}$. Mit a) und b) sind die Positionen zweier Konzentrationsprofile gekennzeichnet, deren Querschnitte in Abbildung 12 zu sehen sind.

Die obere Grafik in Abbildung 11 zeigt das Geschwindigkeitsprofil, die untere Grafik das Konzentrationsprofil für den Fall einer firstparallelen Anströmung eines Traufen-First-Stalles ${ }^{1}$. Den Simulationen liegen Abmessungen eines praxisüblichen Traufen-First-Stalles zugrunde. Die Länges des Stalles beträgt $50 \mathrm{~m}$, die Breite $20 \mathrm{~m}$ und die Firsthöhe 6,60 m. Der Firstschlitz ist 0,2 m breit und durchgehend von einer Giebelwand zur anderen. Der Abstand zwischen Dachinnenseite und den beiden Luftleitplanken (ersichtlich in Abb. 12) beträgt 0,1 m. Dieser Bereich wird beim Einsatz des o.g. Ringleitsystems als Eintrittsort der Frischluft angenommen. Die Luftleitplanken ragen dabei $1 \mathrm{~m}$ in den Stallinnenraum hinein. Im Bodenbereich wird ein Tracer $\left(\mathrm{NH}_{3}\right)$ mit einer Startkonzentration von 20 ppm, gleichmäßig verteilt über die Grundfläche (außer Futtergang), freigesetzt.

Die Luftströmung taucht durch den First in den leeseitig gelegenen Teil des Gebäudes ein. Stallintern

\footnotetext{
${ }^{1}$ Die Simulationsrechnungen für die Abbildungen 11 und 12 wurden mit dem Softwarepaket Coret (ICCM GmbH, Hamburg) durchgeführt.
} 
kommt es zu einer Gegenströmung zur eigentlichen Windanströmrichtung, die einen Austritt des Tracers bzw. der Stallluft aus dem luvseitigen Firstbereich bewirkt. Zu erkennen ist dieser Austritt des Tracers an dem roten Konzentrationsbereich in Abbildung 11 (unten). Beide Grafiken der Abbildung 11 zeigen zudem, dass durch Wirbelbildung im Stall die luvseitig aus dem First austretende Stallluft bzw. der Tracer im weiteren Verlauf erneut durch den First in den Stallraum eindringen kann.

Betrachtet man die beiden Trauföffnungen und die Firstöffnung eines luv- und leeseitigen Querschnittes (Abbildung $12^{2}$ ), so erkennt man ferner, dass die Luftströmung auf der Leeseite zu den Traufen einströmt. Auf der Luvseite kommt es an den Traufen zu einem Stallluftaustritt bzw. zu einem Austritt an Tracer. Das heißt, bei einer firstparallelen Anströmung ist der gesamte Leebereich eines Traufen-First-Stalles ein Lufteintrittsgebiet, der gesamte Luvbereich ein Luftaustrittsgebiet. Das Einbringen von Tracern in die traufseitigen Öffnungen und das Ansaugen der Abluft am First würde zu einer Fehlinterpretation des Abklingverhaltens der Tracerkonzentration und damit des Luftwechsels führen.

Ähnliche Betrachtungen von CHOINIÈRE UND MUNROE [11] an einem Stallmodell bestätigen die Ergebnisse: bei einer Windanströmrichtung parallel zum First wird dieser leeseitig zum Eintrittsort der Frischluft und luvseitig zum Austrittsort der Stallluft. Messkonfigurationen für Traufen-First-Ställe unterliegen damit einer Windrichtungsabhängigkeit und dürfen, unabhängig von einer Berücksichtigung des gezeigten Fehlerpotentials, nur auf diesen Lüftungstyp angewendet werden.

\footnotetext{
${ }^{2}$ Wichtig: den ersichtlichen Farblegenden in Abbildung 12 liegt eine andere Skalierung als in Abbildung 11 zugrunde!
} 

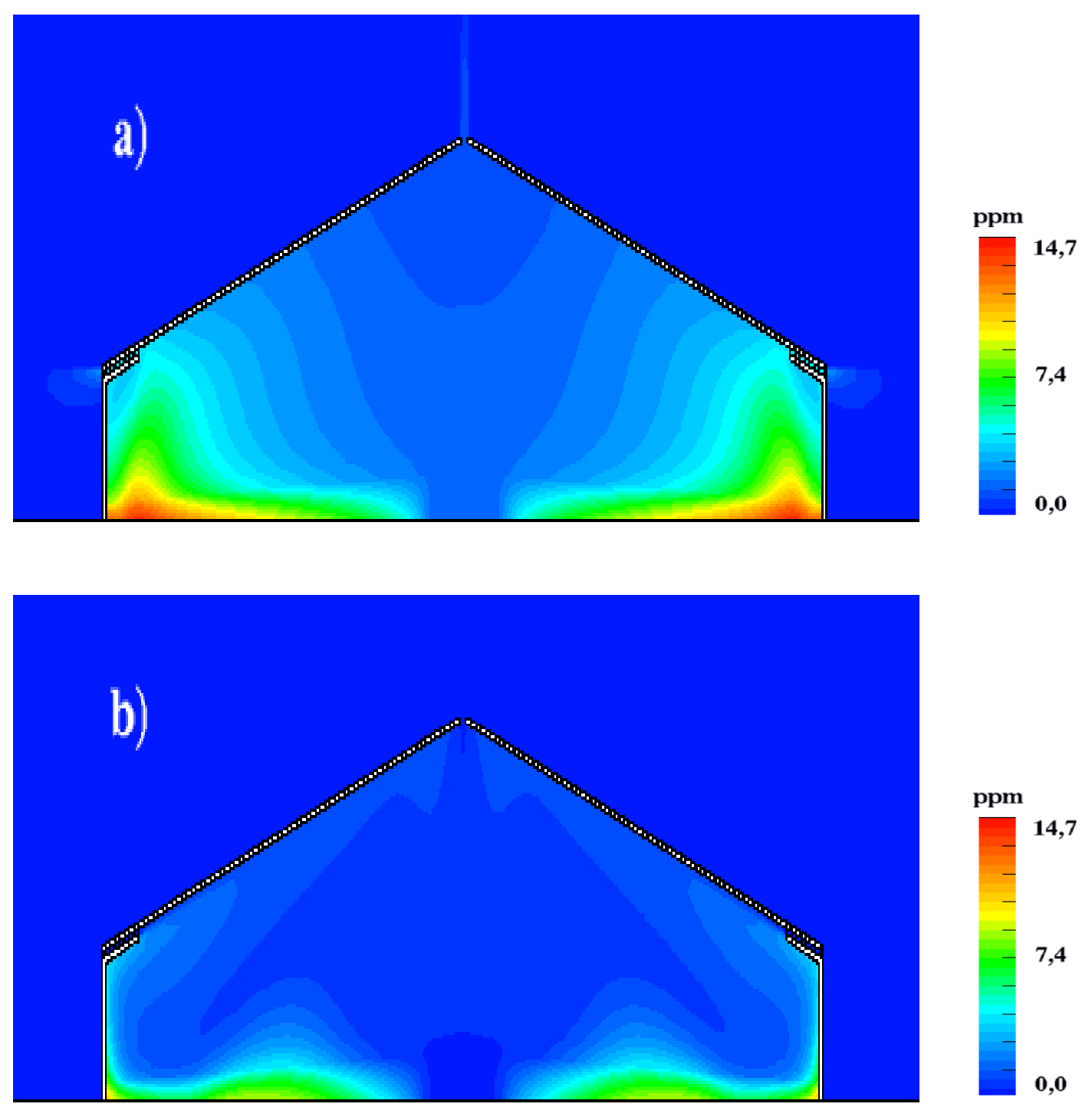

Abbildung 12: Querschnitte zweier Konzentrationsprofile einer firstparallelen Anströmung eines Traufen-First-Stalles. Die Querschnitte beziehen sich auf die in Abbildung 11 mit a) (luvseitig $\Rightarrow$ oben) und b) (leeseitig $\Rightarrow$ unten) eingezeichneten Positionen. Bereiche geringer Konzentrationen sind blau, Bereiche hoher Konzentrationen rot gekennzeichnet. Die Skalierung der Farblegenden ist nicht identisch zur Farblegende in Abbildung 11. 


\subsection{Stoffkonzentrationsbestimmung}

Um Aussagen zum Massenaustrag eines Gases zu machen, ist neben der Berechnung des Luftmassenstromes auch die zeitgleiche Bestimmung der Stoffkonzentration erforderlich. Einmalige, punktuelle Gaskonzentrationsmessungen geben selten Aufschluss darüber, wie hoch die nachhaltige Belastung ist. Deshalb sind Techniken gefragt, die eine kontinuierliche Ermittelung der Stoffkonzentration über einen möglichst langen Zeitraum ermöglichen und die tatsächliche Konzentrationshöhe erfassen. Die Entscheidung fällt damit zugunsten von rechnergestützten-kontinuierlichen Verfahren aus. Im Folgenden werden die wichtigsten Verfahren beschrieben.

\section{Sensoren zur Erfassung der Stoffkonzentration}

Es handelt sich hierbei um chemische Sensoren. Sie werden verwendet, um beispielweise die Änderung des elektrischen Widerstandes eines Halbleiters, in der Regel Silizium, in Kontakt mit der Gasprobe auszunutzen. Diese Sensoren werden als Chemowiderstände bzw. Metalloxidsensoren bezeichnet [61]. Eine modifizierte Form dieser Halbleiterbauelemente ist der Feldeffekttransistor. Sobald die Gasmoleküle mit der Halbleiteroberfläche in Verbindung treten, werden Elektronen zuoder abgeführt. Es kommt zu einer Änderung der elektrischen Leitfähigkeit, welche eine messbare Verschiebung der Schwellenspannung des Feldeffektransistors bewirkt. Die Transistoren sind elektrochemisch so modifiziert, dass nur bestimmte Gasmoleküle, z.B. Ammoniak, hindurchdiffundieren können [36], anhand derer die entsprechende Gaskonzentration ermittelt werden kann.

Des weiteren existieren potentiometrische, kapazitive, optische, calorimetrische und massensensitive chemische Sensoren, deren genaue Einsatzspektren z.B. in [61] ausführlich beschrieben werden.

\section{Konverter}

Konverter für Ammoniak werden meist in Verbindung mit $\mathrm{NO}_{x}$-Analysatoren verwendet. Der Konverter dient der Oxidation von $\mathrm{NH}_{3}$ in der Gasprobe zu NO unter Vorhandensein hoher Temperaturen $\left(750^{\circ} \mathrm{C}\right)$. Das zu NO konvertierte Ammoniak kommt in einen $\mathrm{NO}_{x}$-Analysator (Reaktionskammer). Hier erfolgt eine Chemilumineszenzreaktion ("Lichtemission") zwischen $\mathrm{NO}$ und $\mathrm{O}_{3} \mathrm{zu} \mathrm{O}_{2}$ und angeregtem $\mathrm{NO}_{2}$, wodurch zusätzlich Energie in Form eines Photonenstroms freigesetzt wird. Die Intensität des Photonenstromes ist (bei $p, T=$ konst.) proportional zur Ammoniakkonzentration.

\section{Gaschromatograhie $(G C)$}

Chromatograhie fasst physikalische Vorgänge zusammen, bei denen eine Stofftrennung zwischen einer ruhenden (stationären) und einer sich bewegenden (mobilen) Phase erfolgt. Ein inertes Gas stellt die mobile Phase dar, welches ein Rohr, in dem sich die stationäre Phase befindet, durchströmt. Das zu analysierende Gasgemisch wird mit Hilfe eines sog. Trägergases durch die stationäre Phase transportiert, in der der chromatograhische Vorgang stattfindet. Dies führt zu einem Stromfluss, der wiederum ein elektrisches Signal auslöst, das zur quantitativen Auswertung der Komponenten der Gasprobe 
herangezogen wird [8]. Die Signalfrequenz steigt proportional zur Höhe der detektierten Gaskonzentration.

\section{DOAS (Differentielle Optische Absorptionsspektroskopie)}

Diese Form der Spektroskopie führt Messungen im ultravioletten Bereich (UV-Bereich) durch. Grundlage dieses Verfahrens ist das Lambert-Beersche Gesetz. Ammoniak beispielsweise weist starke Absorptionslinien im UV-Bereich auf. Als Messstrecke dient eine optische Weglänge von bis zu $150 \mathrm{~m}$, die durch Spiegelreflexionssysteme führt. Neben der für $\mathrm{NH}_{3}$ charakteristischen Absorptionswellenlänge wird eine zweite Vergleichswellenlänge gemessen. Über die Differenz der Extinktionen für die beiden Wellenlängen lässt sich die Ammoniakkonzentration der Gasprobe bestimmen.

\section{FTIR (Fourier Transformations Infrarot Spektrometrie)}

FTIR-Techniken bedienen sich einer kontinuierlichen IR-Strahlungsquelle. Die gesamte Strahlung dieser Lichtquelle durchläuft die zu untersuchende Gasprobe und trifft auf eine halbdurchlässige Interferometerplatte, auf der eine Teilung des Lichtstrahls stattfindet. Etwa 50 \% der Strahlung werden zu einem Spiegel reflektiert, der sich mit einer Geschwindigkeit von ca. $1 \frac{\mathrm{cm}}{\mathrm{s}}$ vor und zurück bewegt. Das führt zu einer Überlagerung (Interferenz) der ausgesendeten Strahlung mit der rücklaufenden Strahlung an der Interferometerplatte. Durch diese Überlagerung aller im Spektrum auftretenden Wellenlängen wird ein Interferogramm erstellt [30], anhand dessen durch Fourier-Transformation das entsprechende Gas-IR-Spektrum berechnet und anschließend die Gaskonzentration bestimmt wird.

\section{NDIR (Nicht Dispersive Infrarot Spektrometrie)}

Die NDIR-Spektroskopie findet im Wellenbereich ab ca. $800 \mathrm{~nm}$ Anwendung. Infrarotaktiv sind allerdings nur Moleküle, die durch Rotation oder Schwingung ein stabiles Dipolmoment ${ }^{3}$ aufwei- $^{-}$ sen bzw. bei denen sich während einer Rotation das Dipolmoment ändert [42]. Die Änderung des Dipols kann nur bei bestimmten Frequenzen erfolgen. Stimmt die verwendete Lichtfrequenz (z.B. 10, $6 \mu \mathrm{m} \pm$ 0, 7 für Ammoniak) mit einer der möglichen Dipolfrequenzen der Gasprobe überein, so erfolgt eine Energieabsorption. Je höher die Dipolmomentänderung ist desto höher ist die Energieabsorption und damit die Konzentration des entsprechenden Gases in der Probe.

Eine Spezialform der NDIR-Spektroskopie ist die photoakustische Spektroskopie. Sie wird im Rahmen dieser Arbeit angewendet; weitere Ausführungen zu deren Funktionsweise werden in Kapitel 5.3 unternommen.

\footnotetext{
${ }^{3}$ Anordnung zweier gleich großer elektrischer Ladungen entgegengesetzter Polarität, deren Ladungsschwerpunkte nicht in einem Punkt zusammenfallen
} 


\section{Quantitative Erfassung von Raumluftströmungen}

Die Möglichkeiten zur Vorausbestimmung von Raumluftströmungen sind vielfältig. Je nach Anforderung an das Raumklima oder Problemstellung ist die Quantifizierung und Qualifizierung der Luftführung mit unterschiedlichem Aufwand verbunden.

Man unterscheidet dabei hauptrangig zwischen experimentellen und mathematisch-numerischen Verfahren.

\subsection{Experimentelle Vorgehensweise}

Anhand vorhandener Erfahrungen werden die Art und die Lage der Zuluft- und Abluftöffnungen sowie Zuluftgeschwindigkeiten und Volumenströme festgelegt. Das bedeutet, es werden beobachtete physikalische Erscheinungen gesammelt, ohne diese auf Kausalitäten "abzuklopfen”. Diese Art der Herangehensweise wird als empirisch bezeichnet.

Qualitative Aussagen zu den Strömungsverhältnissen beruhen auf der Sichtbarmachung dieser. Dazu zählen sämtliche Tracerverfahren (Kapitel 2.1.1 bis 2.1.3), so auch einfache Nebel/Rauch-Versuche.

Die quantitative Erfassung von Raumströmungen ist nur durch Messungen von GeschwindigkeitsTemperatur- und/oder Konzentrationsfeldern möglich. Beispiele hierfür sind die Verwendung von Geschwindigkeitssensoren sowie der Einsatz quantifizierbarer Tracertechnik. Mit der Erfassung von Messwerten wird das tatsächliche Geschehen, zumindest für bestimmte Raumbereiche, wiedergegeben.

Werden höhere Anforderungen bezüglich klimatechnischer Parameter (u.a. Stallklimagestaltung) gestellt, so sind experimentelle Untersuchungen der Luftführung in Modellen erforderlich. Die Verwendung von Modellen setzt fundierte Kenntnisse und Vorstellungen über deren Gestaltung und vor allem deren Grenzen voraus [29]. Schwierigkeiten bereitet die unvollständige Ähnlichkeit ("UpScaling”-Problem) eines Modells mit der Originalausführung. Modellversuche erfordern aufgrund ihrer Maßstabsverkleinerung deshalb die Beachtung der Ähnlichkeitstheorie. Wichtige Kennzahlen hierfür sind z.B. die Reynoldszahl Re und die Archimedeszahl Ar.

\subsection{Mathematisch-numerische Vorgehensweise}

Mit Hilfe der ständig steigenden Leistungsfähigkeit von Computern lassen sich Raumluftströmungen auch numerisch berechnen. Dennoch ist es bislang nicht möglich alle elementaren Vorgänge, z.B. das Turbulenzverhalten der Strömung, simulationstechnisch zu erfassen und zu beschreiben [29]. 
Die Strömungsmechanik befasst sich mit der Dynamik der Fluide. Unter Fluiden werden sowohl Gase als auch Flüssigkeiten zusammengefasst, da die meisten strömungsmechanischen Gesetzmäßigkeiten gleichermaßen für beide Stoffe gelten. Um die Bewegung eines Fluids in einer Strömung zu beschreiben, existieren nach [25] zwei verschiedene Ansätze.

Lagrangescher Ansatz:

Dieser Ansatz stammt aus der Punktmechanik. Es wird die Bewegung der einzelnen Teilchen eines Fluids mit der Zeit verfolgt. Der Betrachtungspunkt ist flexibel, er "wandert" mit den Teilchen mit. Der Ansatz wird in der strömungsmechanischen Analytik selten benutzt, da er mit einem großen mathematischen Aufwand verbunden ist. Lagrangemethoden sind vorteilhafter in der Numerischen Strömungssimulation einzusetzen.

\section{Eulerscher Ansatz:}

Es wird die Strömung in einem vorgegebenen Koordinatensystem beschrieben. Jeder Koordinatenpunkt ist durch die Dichte $\rho$, den Druck $p$, die Temperatur $T$ und die Geschwindigkeit $v$ direkt bestimmt. Der Betrachtungspunkt ist fixiert, es interessiert der Wert des gerade am Betrachtungspunkt befindlichen Fluidteilchens. Die zurückgelegte Weglänge der Fluide und deren Anzahl im Raum sind von Bedeutung, nicht das Schicksal eines einzelnen Fluids, immer unter dem Blickwinkel des raumfesten Betrachters.

Die Basis von mathematisch-numerischen Untersuchungen zur Beschreibung eines ebenen ( $i$ bzw. $j=x_{i}, x_{j}$ ) anisothermen Strömungsfeldes bilden die allgemeinen Erhaltungssätze für Masse (Gl. 23), Impuls (Gl. 24) und Energie (Gl. 25):

$$
\begin{aligned}
\frac{\partial\left(\rho \cdot v_{i}\right)}{\partial x_{i}} & =0 \\
\frac{\partial\left(\rho \cdot v_{i}\right)}{\partial t}+\frac{\partial v_{i}}{\partial x_{j}} \cdot\left(\rho \cdot v_{i} \cdot v_{j}\right) & =-\frac{1}{\rho} \cdot \frac{\partial p}{\partial x_{i}}+\frac{\partial}{\partial x_{j}}\left[v \cdot\left(\frac{\partial v_{i}}{\partial x_{j}}+\frac{\partial v_{j}}{\partial x_{i}}\right)\right]+g_{i} \\
\frac{\partial h_{e}}{\partial t}+\frac{\partial h_{e}}{\partial x_{i}} \cdot v_{i} & =-\frac{\partial p}{\partial t}-\frac{1}{\rho} \cdot\left(\frac{\partial q_{i}}{\partial x_{i}}\right)+v_{i} \cdot g_{i}+v \cdot \Phi
\end{aligned}
$$

Die Betrachtung der Massenerhaltung (Kontinuitätsgleichung, Gl. 23) ist die einfachste Vorgehensweise. Es lässt sich stets ein geschlossenes Fluidsystem finden, d.h. ein System, dessen Gesamtmasse konstant ist. Die auf ein Fluidelement wirkenden Massen- und Oberflächenkräfte sowie der molekularbedingte Impulseintrag werden in der Impulserhaltungsgleichung (Gl. 24) beschrieben. Die Gesamtenergie ist die Summe aus der Enthalpie und der kinetischen Energie. Bei Raumströmungen 
kann aufgrund des marginalen Anteils der kinetischen Energie die Gesamtenergie als Enthalpie betrachtet werden [31]. $\Phi$ ist die Dissipationsfunktion, welche die durch innere Reibung irreversibel in fühlbare Wärme umgewandelte mechanische Energie charakterisiert.

Im Rahmen dieser Arbeit wird bei allen durchgeführten Betrachtungen nur auf die Massenerhaltung Bezug genommen.

\subsubsection{Einphasenströmung}

Bei der Betrachtung der Einphasenströmung befindet sich das Fluid als alleinige Substanz in einem beliebig geformten Volumen konstanter Größe. Der Massenstrom $\dot{m}$ des Fluids durch die geschlossene Oberfläche $A$ ist gleich der zeitlichen Änderung der Masse im Volumen $V$ (Abb. 13). Der Ortsvektor der Geschwindigkeit $\vec{v}$ ist senkrecht zur Oberfläche $A$ ausgerichtet.

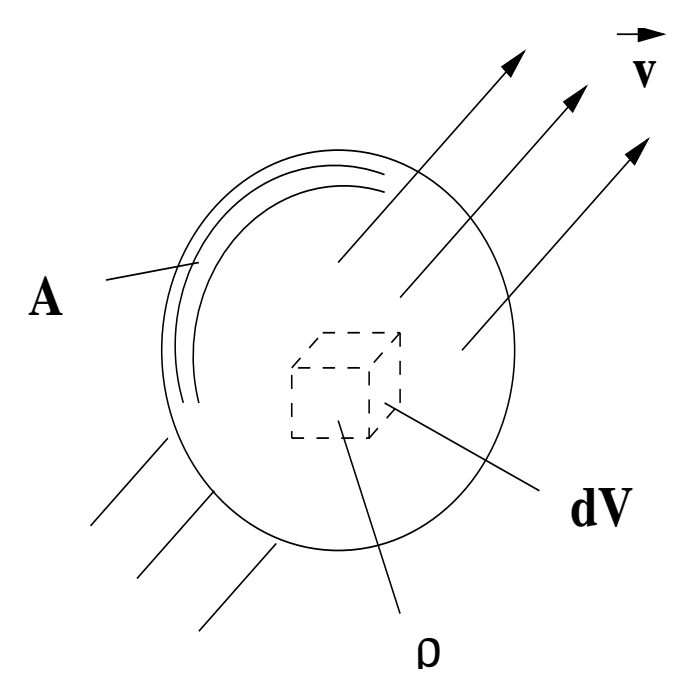

Abbildung 13: Durchströmung eines Volumens V mit der Oberfläche A [22].

Die Beziehung zwischen Massenstrom und Volumenstrom durch eine geschlossene Fläche (Tür, Fenster etc.) lässt sich analog beschreiben:

$$
\begin{aligned}
\dot{m} & =\int_{A}(\rho \cdot v) \cdot d A \\
\dot{V} & =\int_{A} v \cdot d A \\
\int_{A}(\rho \cdot v) \cdot d A & =-\frac{\partial}{\partial t} \int_{V} \rho \cdot d V
\end{aligned}
$$

Gleichung 28 stellt die zuvor aufgeführte Kontinuitätsgleichung in integraler Form dar. Aussagen 
zum Strömungsgeschehen im Inneren eines Systems und damit zu den Bewegungsgleichungen für dreidimensionale Strömungen lassen sich hiermit nicht treffen. Dazu ist es erforderlich, einen Übergang von der integralen Beziehung des Massenaustausches zur differentiellen Beziehung herzuleiten. Wie sieht die Kontinuitätsgleichung für das betrachtete Fluid in differentieller Form aus?

Lässt man ein Raumvolumen unendlich klein werden, so erhält man einen Punkt. Stellt $\vec{r}$ den Ortsvektor eines beliebigen Punktes $R$ im Strömungsfeld dar, dann ist $v(\vec{r})$ mit $\vec{v}=\vec{v}(u, v, w)$ das Geschwindigkeitsfeld von $R$. Die Divergenz des Geschwindigkeitsfeldes $v(\vec{r})$ ist durch Gleichung 29 definiert als

$$
\operatorname{div} v=\lim _{V \rightarrow 0} \frac{\int_{A} v(\vec{r}) \cdot d A}{V}
$$

und damit gleich dem Volumenstrom über der umhüllenden Randfläche $A$ des gegen Null laufenden Volumenelementes. Für $v(\vec{r})$ kann jedes beliebige Vektorfeld stehen. Die Divergenz div des Vektorfeldes $v(\vec{r})$ ist eine skalare Größe und gibt dessen Volumenquellergiebigkeit an. Eine Divergenz $>0$ steht für eine Quelle, eine Divergenz < 0 für eine Senke. Ist die Divergenz $=0$, so ist $v(\vec{r})$ quellfrei, d.h. der Massenstrom im System ist konstant bzw. die Änderung der Masse mit der Zeit ist gleich Null.

Die Beziehung zwischen einem Volumenintegral und einem Oberflächenintegral beschreibt der Gaußsche Integralsatz:

$$
\int_{V} \operatorname{div} v \cdot d V=\int_{A} v \cdot n^{\prime} \cdot d A
$$

$V$ steht für ein Kontrollvolumen, $A$ für die geschlossene Randfläche dieses Volumens und $n^{\prime}$ stellt den nach außen gerichteten Normaleneinheitsvektor von $A$ dar.

Demnach ist die Quellergiebigkeit im Inneren eines Volumens $V$ gleich dem Volumenstrom über der umhüllenden Randfläche $A$ des Volumens. Oder: der Massenaustrag aus einem Volumen ist identisch mit der Abnahme an Masse im Volumen $V$ über dessen Randfläche $A$. Da $v(\vec{r})$ das Geschwindigkeitsfeld einer inkompressiblen Strömung darstellt, ist der Gaußsche Integralsatz identisch zum Massenerhaltungssatz (G1. 28).

Die Übertragung der Gleichung 30 auf ein Fluid mit der Dichte $\rho$ unter Berücksichtigung der Geschwindigkeitskomponenten für die drei Raumanteile liefert

$$
\int_{V} \operatorname{div}(\rho \cdot \vec{v}) \cdot d V=\int_{A}(\rho \cdot \vec{v}) \cdot n^{\prime} \cdot d A
$$

Das Einsetzen von Gleichung 30 in Gleichung 28 ergibt 


$$
\int_{V} \operatorname{div}(\rho \cdot \vec{v}) \cdot d V=-\frac{\partial}{\partial t} \int_{V} \rho \cdot d V
$$

und weiter

$$
\operatorname{div}(\rho \cdot \vec{v})=-\frac{\partial \rho}{\partial t}
$$

Da $\rho(t)=$ konstant gilt die Divergenzfreiheit des Geschwindigkeitsfeldes (div $\vec{v}=0)$ :

$$
\begin{aligned}
\frac{\partial \rho}{\partial t}+\rho \operatorname{div} \vec{v}+\vec{v} \operatorname{grad} \rho & =0 \\
\frac{\partial \rho}{\partial t}+\vec{v} \operatorname{grad} \rho & =0
\end{aligned}
$$

und damit die Kontinuitätsgleichung in Differentialform für ein inkompressibles Fluid mit der Dichte $\rho$. Gleichung 35 fließt zu einem späteren Zeitpunkt (Kapitel 4.3.2) als Bilanzierungsgrundlage für die Luftmassenstromberechnung ein.

\subsubsection{Zweiphasenströmung}

Was passiert, wenn einem Stoff (Trägermedium Luft) ein weiterer Stoff zugesetzt wird? Diese Vorüberlegung ist notwendig für die Durchführung von Tracerexperimenten, da auch ein zugeführter Tracer in Verbindung mit dem Trägermedium als Zweitstoff anzusehen ist. Dabei wird vorausgesetzt, dass der Tracer dem Trägermedium schlupffrei folgt.

Luft setzt sich aus verschiedenen Gasen und Stoffen zusammen. Die sind vor allem Wasser, luftfremde bekannte und unbekannte Stoffe. Die Gesamtmasse z.B. der gasförmigen Stallbestandteile kann mittels folgender Summanden beschrieben werden (Gl. 36):

$$
\begin{aligned}
m_{\text {ges }} & =m_{\text {Luft }}+m_{\mathrm{H}_{2} \mathrm{O}}+m_{\text {Tracer }}+m_{\text {Geruch }}+k \\
& \approx m_{\text {Luft }}\left(1+\frac{m_{\mathrm{H}_{2} \mathrm{O}}}{m_{\text {Luft }}}+\frac{m_{\text {Tracer }}}{m_{\text {Luft }}}+\frac{m_{\text {Geruch }}}{m_{\text {Luft }}}\right)
\end{aligned}
$$

Der Term $\frac{m_{\mathrm{H}_{2} \mathrm{O}}}{m_{\text {Luft }}}$ liegt in der Größenordnung von ca. $10^{-3}$, der Term $\frac{m_{\text {Tracer }}}{m_{\text {Luft }}}$ in der Größenordnung von ca. $10^{-5}$ und der Term $\frac{m_{\text {Geruch }}}{m_{\text {Luft }}}$ in der Größenordnung von ca. $10^{-6} ; k$ steht für eine Konstante. Die Gewichtigkeit der einzelnen Teilmassen auf die Gesamtmasse $m_{\text {ges }}$ lässt sich dann folgendermaßen darstellen (Gl. 38): 


$$
m_{\text {ges }}=m_{\text {Luft }} \cdot\left(1+10^{-3}+10^{-5}+10^{-6}\right) \approx m_{\text {Luft }}
$$

Das heißt, die Bedeutung von Geruchsstoffen oder Tracergas als "Begleitsubstanz" für das Trägermedium Luft ist, bezogen auf die Masse der Luft, vernachlässigbar.

Ein Tracer, der einem System (einmalig) hinzugefügt wird, wird durch die Luftströmung mitgeführt. Der Austausch des Tracers bzw. dessen Ausbreitung im System kann durch Konvektion und/oder Diffusion erfolgen. Die Verteilung des Tracers im Raum wird als inhomogen angenommen. Im Gegensatz zur Luft ist die Dichte des Tracers nicht konstant. Im Hinblick auf die variable Dichte des Tracers ist die Kontinuitätsgleichung (Gl. 35) neu zu definieren.

Strömungen verlaufen laminar oder turbulent. Laminare Strömungen treten im Bereich unterkritischer Geschwindigkeiten auf, die Strömung verläuft schichtenförmig ohne starken Austausch von Fluidteilchen quer zur Strömungsrichtung (Abb. 14a). Beim Überschreiten der kritischen Reynoldszahl $R e_{k r i t}$ schlägt die Strömung vom laminaren in den turbulenten Zustand um (Transition). Für Kanalströmungen liegt $R e_{k r i t}$ bei ca. 2300. Nach dem Strömungsumschlag nimmt die Verteilung der Strömungsgeschwindigkeit ein sehr "bauchiges" Profil an (Abb. 14b). Turbulente Strömungen gehen ohne weitere Energiezufuhr wieder in den laminaren Strömungszustand über.

Die Momentanwerte der Konzentrations- und Geschwindigkeitskomponenten einer stationären turbulenten Strömung lassen sich dem Reynoldsschen Ansatz zufolge in einen zeitlichen Mittelwert $(\bar{C}, \overrightarrow{\vec{V}})$ sowie einen von der Zeit abhängigen Schwankungsteil $\left(c^{\prime}, \overrightarrow{v^{\prime}}\right)$ aufteilen wie in Abbildung 14b ersichtlich:

$$
\begin{aligned}
C & =\bar{C}+c^{\prime} \\
\vec{v} & =\overrightarrow{\vec{V}}+\overrightarrow{v^{\prime}}
\end{aligned}
$$

Die Momentanwerte der Konzentration $C$ und die Komponenten $u, v, w$ des Geschwindigkeitsvektors $\vec{v}$ sind eine Funktion des Ortes und der Zeit, ebenso die Schwankungsgrößen $c^{\prime}$ und $\overrightarrow{v^{\prime}}$. Die Mittelwerte hingegen weisen nur eine Ortsabhängigkeit auf. Der Mittelwert der zeitlich variablen Tracerkonzentration und die zeitlich variablen Schwankungsteile liefern die Kontinuitätsgleichung in folgender Form:

$$
\frac{\partial C}{\partial t}+\operatorname{div}\left(\overline{C \cdot \vec{V}}+\bar{C} \cdot \overrightarrow{v^{\prime}}+c^{\prime} \cdot \overline{\vec{V}}+c^{\prime} \cdot \overrightarrow{v^{\prime}}\right)=0
$$

Durch die Einbringung der Schwankungsgrößen $c^{\prime}, \overrightarrow{v^{\prime}}$ kommen weitere unbekannte Größen hinzu, 


\section{Strömungsarten}

a) laminar
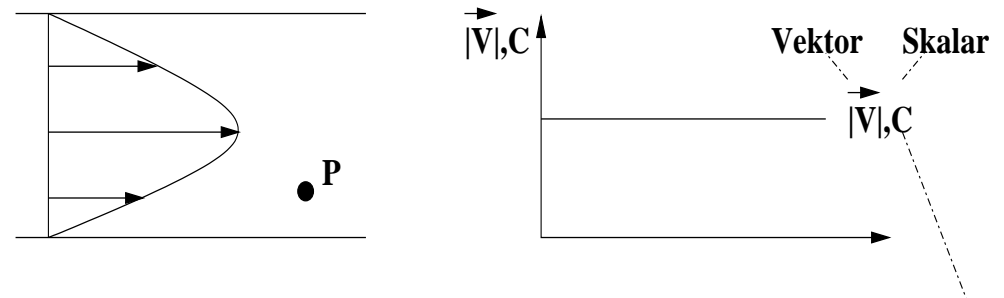

b) turbulent

Mittelwert
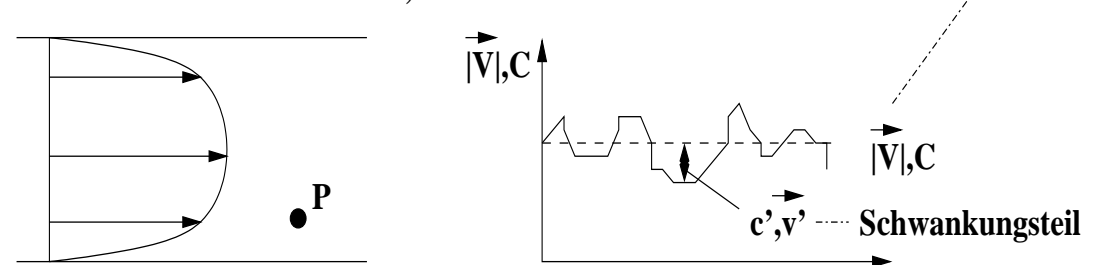

Abbildung 14: Laminare und turbulente Strömung. Punkt $P$ fungiert als trägheitslos arbeitendes Messinstrument in der jeweiligen Strömung. Der Verlauf der Strömung in P ist in den rechten Zeichnungen sichtbar.

welches zu einer Erhöhung der notwendigen Bilanzierungsgleichungen führt. Unter Anwendung der Reynoldsschen Postulate $\left(\overline{a b^{\prime}}=0 ; \overline{a^{\prime} b}=0 ; \overline{a^{\prime} b^{\prime}} \neq 0 ; \overline{a b} \neq 0, a\right.$ und $b$ sind Variablen) vereinfacht sich Gleichung $41 \mathrm{zu}$

$$
\frac{\partial C}{\partial t}+\operatorname{div}\left(\overrightarrow{C \cdot \vec{V}}+c^{\prime} \cdot \overrightarrow{v^{\prime}}\right)=0
$$

Der Schwankungsteil $c^{\prime}, \overrightarrow{v^{\prime}}$ beinhaltet Aussagen zur statistischen Abhängigkeit (Korrelation) der einzelnen Parameter:

$E\left(c^{\prime} \cdot \overrightarrow{v^{\prime}}\right)=0: c^{\prime}$ und $\overrightarrow{v^{\prime}}$ sind statistisch unabhängig

$E\left(c^{\prime} \cdot \overrightarrow{v^{\prime}}\right) \neq 0: c^{\prime}$ und $\overrightarrow{v^{\prime}}$ korrelieren

In Fall einer Zweiphasenströmung ist $E\left(c^{\prime} \cdot \overrightarrow{v^{\prime}}\right) \neq 0$, d.h. der zeitliche Verlauf der Konzentration eines Tracers weist - zumindest zeitweise - eine Ähnlichkeit mit dem Geschwindigkeitsverlauf des Trägermediums Luft auf. Das Produkt $\overrightarrow{C \cdot \vec{V}}$ drückt den konvektiven Austausch des Tracers infolge aktiver Strömungsmitnahme aus, $c^{\prime} \cdot \overrightarrow{v^{\prime}}$ ist als diffusiver Austausch aufgrund vorherrschender Konzentrationsunterschiede des Tracers im Volumenelement anzusehen. Mit Hilfe der Lösung erster Näherung, auch als Gradientenansatz bezeichnet [38], lässt sich folgende Beziehung aufstellen:

$$
c^{\prime} \cdot \overrightarrow{v^{\prime}}=D_{x x} \operatorname{grad} C
$$


$D_{x x}$ wird als Diffusionskoeffizient bezeichnet, er weist die Dimension 'Länge ${ }^{2} / Z e i t$ ' auf. Er ist ein Tensor (mathematisches Konstrukt) und beinhaltet alle bekannten und unbekannten Schwankungsgrößen, die sich nicht erfassen lassen. Im Unterschied zum zweiten Fickschen Gesetz (G1. 44) ist $D_{x x}$ eine reine Strömungsgröße. Der molekulare Diffusionskoeffizient $D_{m}$ des Fickschen Gesetzes hingegen ist eine stoffabhängige Größe!

$$
\begin{aligned}
\frac{\partial C}{\partial t} & =-D_{m}\left(\frac{\partial^{2} C}{\partial x^{2}}+\frac{\partial^{2} C}{\partial y^{2}}+\frac{\partial^{2} C}{\partial z^{2}}\right) \\
& =-D_{m} \triangle C
\end{aligned}
$$

Das negative Vorzeichen in Gleichung 44 charakterisiert die Bewegung des Stoffes von Bereichen hoher Konzentration hin zu Bereichen niedriger Konzentration.

Unter Berücksichtigung der turbulenten und molekularen Diffusion des Tracers innerhalb des Trägermediums Luft sowie der Reynoldsschen Postulate ergibt sich aus den Gleichungen 42 und 43 die Kontinuitätsgleichung für einen Tracer inkonstanter Dichte in einer Zweiphasenströmung (partielle Massenerhaltungsgleichung) zu:

$$
\frac{\partial C}{\partial t}+\operatorname{div}\left(C \cdot \vec{V}+D_{x x} \operatorname{grad} C\right)=0
$$

Aus Gründen der Übersichtlichkeit werden von nun an die Querstriche der Mittelwertgrößen weggelassen. Aufgelöst ergibt sich Gleichung 47

$$
\frac{\partial C}{\partial t}+\operatorname{div}(C \cdot \vec{V})+\operatorname{div}\left(D_{x x} \operatorname{grad} C\right)=0
$$

und unter Anwendung des Greenschen Satzes erhält man Gleichung 48

$$
\frac{\partial C}{\partial t}+C \operatorname{div} \vec{V}+\vec{V} \operatorname{grad} C+\operatorname{div}\left(D_{x x} \operatorname{grad} C\right)=0
$$

Da $\operatorname{div} \vec{V}=0$ und $D_{x x} \operatorname{div} \operatorname{grad} C=D_{x x} \nabla(\operatorname{grad} C)=D_{x x} \triangle C$ folgt

$$
\begin{aligned}
\frac{\partial C}{\partial t}+\vec{V} \operatorname{grad} C+\operatorname{div}\left(D_{x x} \operatorname{grad} C\right) & =0 \\
\frac{\partial C}{\partial t}+\vec{V} \operatorname{grad} C+D_{x x} \operatorname{div} \operatorname{grad} C+\operatorname{grad} C \operatorname{grad} D_{x x} & =0 \\
\frac{\partial C}{\partial t}+\operatorname{grad} C \cdot\left(\vec{V}+\operatorname{grad} D_{x x}\right)+D_{x x} \triangle C & =0
\end{aligned}
$$




$$
\frac{\partial C}{\partial t}+\operatorname{grad} C \cdot\left(\vec{V}+\overrightarrow{V_{d i f f}}\right)+D_{x x} \triangle C=0
$$

$\overrightarrow{V_{\text {diff }}}$ stellt den Geschwindigkeitsvektor des diffusiven Stofftransportes dar, der aus der vorherrschenden Konzentrationsdifferenz zwischen zwei Raumpunkten resultiert, $\vec{V}$ den Geschwindigkeitsvektor des konvektiven Stofftransportes. Im Konvektionsfall werden Funktionswerte betrachtet, im Diffusionsfall Gradienten. Entscheidend für eine spätere mathematische Lösung des partiellen Differentialgleichungssystems ist die Proportion zwischen konvektivem und diffusivem Stofftransport, wobei im Allgemeinen der konvektive Transport überwiegt d.h. $V \gg V_{\text {diff }}$. Gleichung 52 ist bei Annahme von $D_{x x}=0$ mit $\overrightarrow{V_{d i f f}}=\operatorname{grad} D_{x x}$ identisch zu Gleichung 35 und damit als Näherungslösung anzusehen. Unter dieser Bedingung werden die Berechnungen der vorliegenden Arbeit durchgeführt.

Bei Stallum- und Stalldurchströmungen ist aufgrund der Böigkeit des Windes und der vorhandenen Ablösekanten (Ecken des Stallgebäudes) meist eine turbulente Strömung vorzufinden. Die eingebrachte Tracerkonzentration unterliegt deshalb hauptsächlich dem turbulenten Diffussionsaustausch; der molekulare Diffussionsaustausch $\left(D_{x x} \operatorname{grad} C\right.$ ) kann in der Regel vernachlässigt werden. In Systemrandgebieten (Fenster- und Türöffnungen etc.) muss auch ein unkontrollierter Massenaustausch infolge Diffusion angenommen werden $\left(D_{x x} \neq 0\right)$. $D_{x x}$ kann hier als Funktion der Zeit und des Ortes angesehen werden. Möglichkeiten, diesen Einfluss näher zu untersuchen, bestehen in skalaren Verfahren, von denen in den Abschnitten 3.4.1 und 3.4.2 zwei ausgewählte vorgestellt werden.

\subsection{Numerische Strömungssimulation}

Die mathematisch-numerische Quantifizierung von Strömungsprozessen unterliegt den Erhaltungssätzen für Masse, Impuls und Energie. Eine analytische Lösung dieser partiellen, nichtlinearen und gekoppelten Gleichungen ist bislang nur in wenigen einfachen Spezialfällen gelungen [29]. In der Numerik werden Algorithmen angewendet, die zu einer näherungsweisen Lösung von Modellgleichungen mittels einfacher arithmetischer Operationen an definierten (diskreten) Stellen im Lösungsgebiet führen. Hierfür stehen Lösungsverfahren zur Verfügung (siehe Abschnitte 3.3.1ff), die sich hauptsächlich in der Ausgangsform der Gleichungen und in den Näherungssätzen unterscheiden.

Numerische Modelle dienen der Visualisierung des Strömungsgeschehens (siehe auch Abbildung 4) und sind als Hilfsmittel der Strömungsmechanik zu betrachten, um ein "Gefühl” über die prinzipiellen Strömungsvorgänge zu erhalten. In vielen Fällen führen numerische Verfahren überhaupt erst zu einer Lösung eines Problems. In anderen Fällen beschleunigen sie die Lösungsfindung, allerdings auf Kosten der Genauigkeit, denn ein Modell beruht stets auf Vereinfachungen. Die häufigsten Fehlerquellen numerischer Modelle sind Diskretisierungsfehler (Kapitel 3.3.1) und Iterationsfehler.

Durch Berücksichtigung der Erhaltungsgleichungen wird sichergestellt, dass sich die Lösung einer 
numerischen Simulation innerhalb der physikalischen Grenzen befindet. Ebenso können, im Vergleich zu Experimenten, die Randbedingungen (Begrenzungen des untersuchten Strömungsgebietes) realistischer simuliert werden, da Leckagen o.ä. ausgeschlossen sind. Die gesamte Informationsbasis ist genauer und vollständiger. Unzulänglichkeiten weisen mathematische Modelle vor allem in der Berechnung des Turbulenzverhaltens sowie durch ungenaue numerische Approximationen auf. Als weiterer Nachteil muss die begrenzte Rechnerhardware aufgeführt werden, die trotz der derzeitigen Verfügbarkeit hochleistungsfähiger Computer den Rahmen der Berechnungsmöglichkeiten erheblich einschränkt.

Es werden nun drei wichtige und verbreitete dreidimensionale Berechnungsverfahren der numerischen Strömungssimulation vorgestellt, die z.B. im Rahmen von CFD (Computational Fluid Dynamics) angewendet werden können. Maßgebend ist in der Regel die Möglichkeit der numerischen Diskretisierung. Sie entscheidet darüber, welche Berechnungsrichtung eingeschlagen wird. Beispielanwendungen der CFD-Simulation finden sich u.a. in [46] und [68].

\subsubsection{Finite Differenzen Verfahren}

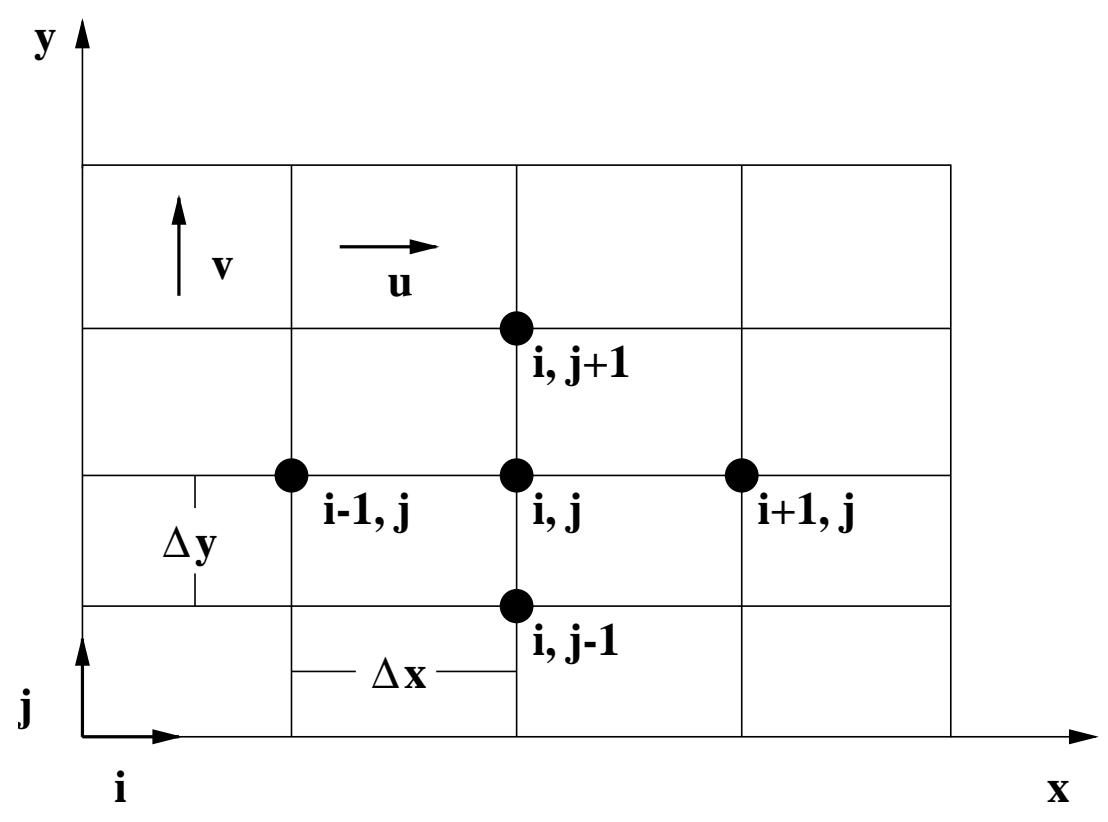

Abbildung 15: Gitternetz für ein betrachtetes Strömungsgebiet mittels Finite Differenzen Verfahren im zweidimensionalen Fall. Alle strömungsrelevanten Größen werden an den Gitterpunkten des Gitternetzes ermittelt.

Die numerische Berechnung des Differentialgleichungssystems der aufgeführten Grundgleichungen (Gln. 23, 24 und 25) beruht auf der Approximation durch Differenzengleichungen. Dazu wird das betrachtete Strömungsgebiet mit einem mehr oder weniger feinmaschigen Gitternetz überzogen, sprich diskretisiert, welches im einfachsten Fall eine Rechtecksform - äquidistantes Gitter - besitzt (Abb. 
15 für einen zweidimensionalen Fall). Die Linien des Gitters können als Koordinatenlinien betrachtet werden. Man erhält eine endliche (=finite) Zahl von Gitterpunkten, an denen die abhängigen Variablen definiert sind und z.B. in eine Taylorreihe entwickelt werden können [57]. Die Differentiale werden an den Gitterpunkten $i, j$ durch Differenzen ersetzt, dazu müssen die Approximationen für die Ableitungen in den Gitterpunkten gewählt werden. Der Fehler, der durch den Ersatz des Kontinuums durch ein diskretes Modell entsteht, wird als Diskretisierungsfehler bezeichnet.

Für den in Abbildung 15 betrachteten zweidimensionalen Fall läuft $i$ in x-Richtung und $j$ in y-Richtung. Die Position der Gitterpunkte ist durch Indizierung eindeutig festgelegt. Zwischen den Gitterpunkten muss interpoliert werden, da die Näherungslösung nur für die Gitterpunkte existiert. Für jeden Gitterpunkt $i, j$ wird nun die gegebene Differentialgleichung in eine Differenzengleichung umgewandelt. Entsprechend der Anzahl der zur Berechnung verwendeten Differentialgleichungen wird die gleiche Anzahl an Differenzengleichungen gewonnen, die im Allgemeinen miteinander gekoppelt sind.

Eine Verfeinerung des Gitters führt zu einer restriktiven Zeitschrittbegrenzung, d.h. bei der Wahl der Zellenabmessungen $\Delta x$ und $\Delta y$ sowie des Zeitschritts $\Delta t$ müssen bestimmte Bedingungen eingehalten werden. Diese Bedingungen sind völlig unabhängig vom tatsächlichen zeitlichen Verlauf der Lösung [21]. Das bedeutet beispielsweise: der Zeitschritt $\Delta t$ muss so gewählt werden, dass die mit dem Geschwindigkeitsvektor $\vec{v}$ transportierte Verteilung von $C$ pro Zeitschritt höchstens einen Gitterpunkt vorankommt:

$$
\Delta t<\min \left[\frac{\Delta x}{|u|} ; \frac{\Delta y}{|v|} ; \frac{\Delta z}{|w|}\right]
$$

Beim Finite Differenzen Verfahren wird durch die Diskretisierung das kontinuierliche Differentialgleichungssystem somit auf ein diskretes Differenzengleichungssystem reduziert, das vom Rechner gelöst werden kann [17].

Raumströmungen sind stets dreidimensional. In erster Näherung werden im Allgemeinen zweidimensionale Betrachtungen durchgeführt. Es besteht aber die Gefahr der Vernachlässigung wichtiger Bereiche bzw. Kriterien, welche der eigentlichen dreidimensionalen Strömung anhaften, die unter Umständen nur vereinfacht oder gar nicht berücksichtigt werden.

\subsubsection{Finite Volumen Verfahren}

Beim Finite Volumen Verfahren werden Approximationen für die Flächen- und Volumenintegrale sowie Variablenwerte und Ableitungen an verschiedenen Stellen definiert. Eine Berechnung der Flächen- und Volumenintegrale ist ebenfalls nur numerisch möglich, da die räumliche Variation der 
zu integrierenden Funktion unbekannt ist [41]. Im Unterschied zum Finite Differenzen Verfahren, welches eine näherungsweise Lösung für ein Differentialgleichungssystem liefert, wird der Erhaltungssatz für die Masse in einem Bezugsraum im integralen Sinn erfüllt.

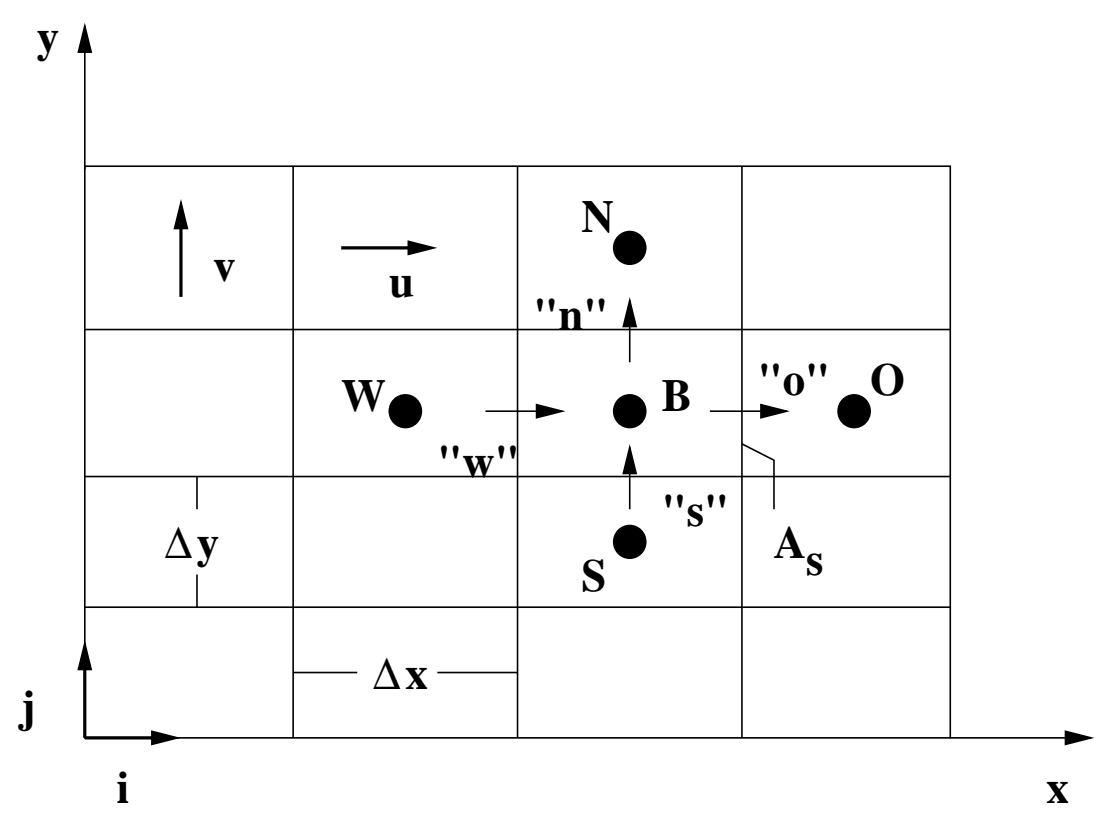

Abbildung 16: Gitternetz für ein betrachtetes Strömungsgebiet mittels Finite Volumen Verfahren im zweidimensionalen Fall.

Das betrachtete Strömungsgebiet wird in eine endliche Zahl von Kontrollvolumina aufgeteilt, die sich nicht überschneiden (Abb. 16), wobei die Erhaltungsgleichungen für jedes dieser Volumina angewendet werden. Die beschreibenden Variablen sind im Innern eines Kontrollvolumens definiert und nicht an den Schnittstellen, sprich virtuellen Austauschflächen.

Der Stoffaustausch zwischen zwei Kontrollvolumina wird mittig über die Seitenflächen angenommen. Im einfachsten zweidimensionalen Fall weist ein Kontrollvolumen vier Seitenflächen auf. Neuere Ansätze gehen von beliebig vielen Seitenflächen aus, wobei lediglich die Oberflächennormalenvektoren eines Volumenelementes sowie die jeweiligen Verbindungen zu den benachbarten Elementen interessieren [56]. Für die Flussbilanz im betrachteten Kontrollvolumen um $B$ ergibt sich

$$
\dot{m}_{o}-\dot{m}_{w}+\dot{m}_{n}-\dot{m}_{s}=0
$$

Die Summe der Integrale aller Seitenflächen eines Kontrollvolumens ist gleich dem Integral über die gesamte geschlossene Oberfläche dieses Kontrollvolumens: 
im Konvektionsfall

$$
\int_{A}(C \cdot \vec{v}) \cdot n^{\prime} \cdot d A=\sum_{S} \int_{A_{S}}(C \cdot \vec{v}) \cdot n^{\prime} \cdot d A
$$

im Diffusionsfall

$$
\int_{A} D \operatorname{grad} C \cdot n^{\prime} \cdot d A=\sum_{S} \int_{A_{S}} D \operatorname{grad} C \cdot n^{\prime} \cdot d A
$$

Der sog. "computational node", der Bezugspunkt $B$ für alle unbekannten Größen (Abb. 16), wird im Zentrum eines jeden Kontrollvolumens angenommen [41]. Der Wert des Bezugspunktes (hier: Dichte bzw. Konzentration $C$ ) ist bekannt, die $C$-Werte an den Volumenoberflächen und im Volumen selbst unbekannt. Sie müssen durch Interpolation bestimmt werden, d.h. es müssen für diese Volumen- und Oberflächenbereiche Näherungen getroffen werden.

Die einfachste Möglichkeit zur Approximation des Flächenintegrals besteht darin [21], den Wert der zu integrierenden Funktion in der Mitte der Seitenfläche $A_{S}$ des Kontrollvolumens als Mittelwert über die gesamte Seitenfläche anzusehen

$$
\int_{A_{S}}(C \cdot \vec{v}) \cdot n^{\prime} \cdot d A=\overline{\left[(C \cdot \vec{v}) \cdot n^{\prime}\right]_{S}} \int_{A_{S}} d A
$$

Das heißt, der Wert von $C$ ist an jedem Punkt einer Seitenfläche gleich.

Die numerische Berechnung des Volumenintegrals wird ähnlich durchgeführt: es wird angenommen, dass der Wert der zu integrierenden Größe $(C)$ im Bezugspunkt $B$ den Mittelwert über das gesamte Kontrollvolumen darstellt

$$
C_{B}=\int_{V} \bar{C} \cdot d V
$$

Finite Volumen Verfahren sind konservative Berechnungsmethoden, da die Formulierung der Erhaltungsgleichungen auf eine feste (kartesische) Basis bezogen wird. Die Erhaltungsgleichungen für das betrachtete Strömungsgebiet ergeben sich damit aus der Summe der Gleichungen über alle Kontrollvolumina. Das heißt, die Integrale über die inneren Seitenflächen heben sich auf, es treten nur die Flächenintegrale über die gesamten Randflächen des Strömungsgebietes sowie die Summe der Volumenintegrale auf [55]. Die in Abschnitt 3.3.1 erwähnten Diskretisierungsfehler können sich im System nur durch falsche Umverteilung, verzerrte Profile etc. bemerkbar machen, die betrachtete physikalische Größe $\bar{C}$ bleibt erhalten.

Finite Volumen Verfahren können bei beliebig geformten Kontrollvolumina angewendet werden. Die einfachste Betrachtungsform ist die geschilderte Rechtecksform im zweidimensionalen bzw. Hexa- 
ederform im dreidimensionalen Fall. Die Gittergenerierung ist dabei der entscheidende Teil. Grundlage der Berechnung in dieser Arbeit wird die Verwendung des hier beschriebenen Verfahrens sein, worauf ab Kapitel 4.3 zurückgegriffen wird.

\subsubsection{Finite Elemente Verfahren}

Bei der Methode der finiten Elemente wird das Strömungsgebiet in Teilbereiche mehr oder weniger beliebiger Form zerlegt [44]. Diese Teilbereiche werden als finite Elemente, die Eckpunkte der Teilbereiche als Knoten bezeichnet. Im Gegensatz zu den Approximationen für die Ableitungen bzw. Flächen- und Volumenintegrale der beiden zuvor behandelten Verfahren werden hier Formfunktionen gewählt und keine Bilanzierungen vorgenommen.

Mit Hilfe von Reihenansätzen wird beispielsweise die Differentialform der Erhaltungsgleichungen für jedes Element näherungsweise beschrieben. Die Differentialgleichung ist gleichzeitig die Bilanzgleichung eines jeden Elementes. Im Allgemeinen wird die Näherungslösung die ursprüngliche Bilanzgleichung nicht exakt erfüllen, es verbleibt ein Approximationsfehler (Residuum $R$ ). Durch die Formfunktion wird nur die Abhängigkeit von Knotenwerten zueinander spezifiert; das Residuum wird als Funktion der unbekannten Knotenwerte angesehen.

Die Lösung des Gleichungssystems erfolgt unter der Bedingung der Orthogonalität des Residuums zu einer Basis linearer unabhängiger Wichtungsfunktionen $\vec{w}[39] \mathrm{mit}$

$$
\int_{V} \vec{w} \cdot R \cdot d V \rightarrow \min
$$

$\vec{w}$ sind die Wichtungsfunktionen, mit denen das Residuum von jedem Volumenelement gewichtet wird. Der Fehler der Näherungslösung im integralen Mittel wird über das betrachtete Strömungsgebiet $V$ minimiert.

Mit Hilfe der Methode der gewichteten Residuen kann eine Differentialgleichung in eine entsprechende Funktionalgleichung überführt werden. Die Lösung dieser Funktionalgleichungen kann durch verschiedene Verfahren erzielt werden. Stellvertretend seien hier die Galerkin-Methode und die PetrovGalerkin-Methode (Upwind-Verfahren) genannt.

Für weiterführende Betrachtungen wird auf das sehr ausführliche Werk von Bathe [5] verwiesen.

\subsection{Skalare Lösungsverfahren}

Skalare Verfahren zur Quantifizierung von Strömungen sind als eindimensionale Methoden einzuordnen. Im Unterschied zu den behandelten dreidimensionalen Lösungsverfahren werden hier absolute 
Größen gemessen. Aussagen zum internen Strömungsverhalten sind nicht möglich. Die Basis von skalaren Verfahren stellt die Kontinuitätsgleichung (Gl. 23) dar, welche besagt, dass im stationären Fall die einströmende Masse eines inkompressiblen Fluids gleich der ausströmenden Masse des Fluids ist.

\subsubsection{Druckdifferenzmessungen}

Strömungen verlaufen stets entlang eines negativen Druckgradienten, d.h. vom Bereich höheren Druckes zum Bereich niedrigeren Druckes. Die Durchströmung eines Gebäudes ist das Ergebnis der Wirkung von Druckquellen und Drucksenken. In Stallgebäuden mit natürlicher Lüftung wirken der thermische Auftrieb und die Windströmung als Druckquellen, First-, Seitenwand-, Fenster- und Türöffnungen etc. als Drucksenken. Druckunterschiede entstehen durch die Umströmung des Stalles [37].

Ziel von Druckdifferenzmessungen ist es, anhand von Druckmessungen im Stall und in den Öffnungen eine mittlere Geschwindigkeit durch diese Öffnungen zu berechnen. Mit Hilfe von Druckdifferenzmessungen kann somit auch festgestellt werden, ob eine Öffnung als $\mathrm{Zu}$ - oder Abluftöffnung fungiert bzw. keinen Einfluss auf die Luftaustauschvorgänge in einem Gebäude ausübt. Eine der Hauptschwierigkeiten besteht dabei in der Bestimmung des stallinternen Druckkoeffizienten $c_{p i}$ [52], der als Bezugsdruck (Bezugsniveau) benötigt wird, sowie des Druckkoeffizienten $c_{p A}$ in der jeweils betrachteten Öffnung. $c_{p i}$ wird von der Lage der sog. Neutralen Zone (Abb. 17) beeinflusst. Sie wird als das fiktive Niveau bezeichnet, auf welchem der Stallinnendruck gleich dem Außendruck ist, das Einströmen der Luft in ein Ausströmen übergeht und umgekehrt [29].

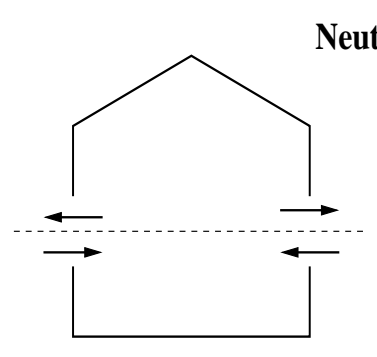

a)

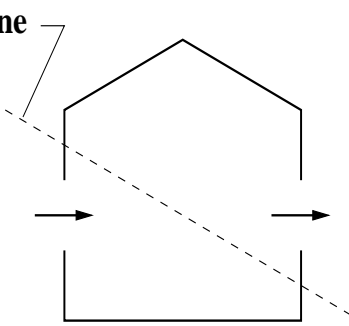

b)

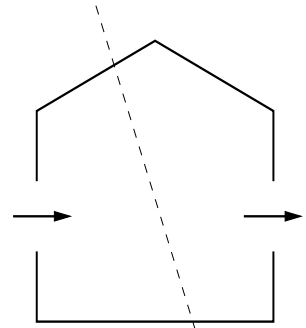

c)

Abbildung 17: Lage der Neutralen Zone bei verschiedenen Windgeschwindigkeiten: a) Windstille, b) geringe Windgeschwindigkeit, c) hohe Windgeschwindigkeit.

Die Lage der neutralen Zone ist zum einen abhängig von den vorherrschenden Windverhältnissen (Abb. 17): je größer die Windgeschwindigkeit desto steiler ist die neutrale Zone ausgerichtet; bei Windstille liegt sie waagerecht. Zum anderen beeinflussen Größe und Position der Stallöffnungen die neutrale Zone. Durch Öffnungen oberhalb der neutralen Zone strömt die Luft hinaus, unterhalb 
herein unter der Voraussetzung, dass die Außendrücke, z.B. am Dach, nicht durch zusätzliche Öffnungen beeinflusst werden. Die Durchströmgeschwindigkeit einer Öffnung steigt mit zunehmender Entfernung von der neutralen Zone an. Befindet sich eine Öffnung in unmittelbarer Nähe dieser Zone, so tendiert deren Durchströmgeschwindigkeit und damit deren Wirkung gegen Null. Durch Schließen bzw. Öffnen der Zu- und Abluftöffnungen (Jalousien, Tore, Türen etc.) können die Druckverhältnisse und damit die Lage der neutralen Zone beeinflusst werden.

Wie oben bereits erwähnt, stellen die Windströmung und der thermische Auftrieb die beiden wesentlichen Einflussfaktoren bei der Durchführung von Druckdifferenzmessungen dar. In der Regel tritt eine Kombination beider Größen auf. Bei Annahme einer konstanten Windgeschwindigkeit $v_{A}$ in einer Stallöffnung bekannter Höhe kann der daraus resultierende Druck über die gesamte Querschnittsfläche $A$ nach Bernoulli wie folgt beschrieben werden (Gl. 58):

$$
p_{w}=\frac{1}{2} \cdot \rho \cdot v_{A}^{2}
$$

Unter Berücksichtigung der Druckkoeffizienten stallintern $c_{p i}$ und in den Öffnungen $c_{p A}$ folgt aus Gleichung 58

$$
\Delta p_{w}(x, y, h)=\frac{1}{2} \cdot \rho_{a u ß e n} \cdot v_{A}^{2} \cdot\left[c_{p A}(x, y, h)-c_{p i}\right]
$$

$\Delta p_{w}$ stellt den statischen Druck (Winddruck) der ungestörten atmosphärischen Strömung, $\rho_{a u ß e n}$ die Dichte der Außenluft dar. Infolge der gleichzeitigen Überströmung des Gesamtgebäudes existiert der dynamische Druck $p_{U}$. Das Verhältnis beider Drücke wird als Widerstandsbeiwert $c_{w}$ bezeichnet (Gl. 59); er ist dimensionslos und wird experimentell in Windkanalversuchen ermittelt [34]:

$$
c_{w}=\frac{p_{w}}{p_{U}}
$$

Anhand der gemessenen Druckdifferenzen zwischen statischem und dynamischem Druck und der im weiteren Verlauf resultierenden Geschwindigkeiten kann, bei konstanter Dichte $\rho_{a u ß e n}$ der Außenluft, der Luftvolumenstrom $(v \cdot A)$ berechnet werden.

Bei Berïcksichtigung des thermischen Auftriebs im System, der durch vertikale Temperaturunterschiede hervorgerufen wird, lässt sich die Druckdifferenz $\Delta p_{\text {therm }}$ (Gl. 60) ebenfalls bestimmen [45],[52]:

$$
\Delta p_{\text {therm }}(h)=\left(\rho_{\text {außen }}-\rho_{\text {innen }}\right) \cdot g \cdot\left(h_{N Z}-h\right)
$$


$h_{N Z}$ charakterisiert dabei die Lage der Neutralen Zone, vermindert um die Höhe $h$ der betrachteten Temperaturebene.

Nach Bestimmung der Druckkoeffizienten $c_{p i}$ und $c_{p A}$ kann die mittlere Geschwindigkeit $\overline{v_{A}}$ durch eine horizontale oder vertikale Querschnittsfläche $A$ wie folgt berechnet werden (Gl. 61):

$$
\overline{v_{A}}=v \cdot \frac{\left|c_{p A}-c_{p i}\right|^{\frac{3}{2}}}{c_{p A}-c_{p i}} \cdot \sqrt{\frac{\rho_{a u \beta e n}}{\rho_{A} \cdot E_{A}}}
$$

$v$ kennzeichnet die Windgeschwindigkeit außerhalb des Stalles. Für Windgeschwindigkeiten, die in den Stall gerichtet sind, ist $\overline{v_{A}}>0$, im Fall aus dem Stall gerichteter Windgeschwindigkeiten ist $\overline{v_{A}}<0$. Die vorhandenen Druckdifferenzen infolge der Um- und Durchströmung eines Gebäudes sind auch von der Größe der jeweiligen $\mathrm{Zu}$ - und Abluftöffnungen abhängig. $E_{A}$ stellt die Eulersche Zahl dar, in der die Ausprägung der jeweils betrachteten Öffnung (Windnetz, Spaceboard, angekipptes Fenster etc.) berücksichtigt wird [52]. Unter Umständen ist es erforderlich, für bestimmte Lagen der Neutralen Zone (Abb. 17a)) zwei mittlere Geschwindigkeiten für eine Öffnung zu berechnen: eine Geschwindigkeit für den oberen und eine für den unteren Teil der Öffnung. Eine detailliertere Betrachtung führt aber an dieser Stelle zu weit.

Neben der oben geschilderten Vorgehensweise kann der Volumenstrom auch direkt mit Hilfe eines Potenzansatzes (Gl. 62) als Funktion der Druckdifferenz dargestellt werden [29]:

$$
\dot{V}=k \cdot \Delta p^{n_{S}}
$$

$k(\approx 0,5)$ ist der Durchflusskoeffizient, d.h. die absolute Größe des effektiven Strömungsquerschnittes [60] und $\Delta p$ die Druckdifferenz zwischen innen und außen. $n_{S}$ stellt den dimensionslosen Strömungsexponenten dar, er charakterisiert die Strömungsart

$n_{S}=0,5 \Rightarrow$ turbulent

$n_{S}=1,0 \Rightarrow$ laminar

Trotz Berücksichtigung der (vertikalen) Auftriebsgeschwindigkeit, der (horizontalen) Windgeschwindigkeit sowie der Kontinuitätsbedingung für die Luft existiert derzeit kein Referenzverfahren für die Druckdifferenzmessung [52]. Als Hauptursache wird der unzureichend geklärte Einfluss von weiteren Gebäuden und Bewuchs in der unmittelbaren Nähe des untersuchten Systems angesehen, infolgedessen das Druckniveau im System bei wechselnden Windanströmrichtungen beeinflusst wird. 


\subsubsection{Maschenverfahren}

Weist ein Gebäude neben großflächigen Öffnungen weitere wesentliche Zu- und Abluftöffnungen auf, so kann in erster Näherung ein Maschenverfahren angewendet werden [15]. Diese Methode spielt vor allem in Industrie- und Bürogebäuden eine große Rolle, im Bereich der Landwirtschaft kann z.B. ein Tandem-Stall (zwei benachbarte Ställe, die lüftungstechnisch miteinander verbunden sind) für ein derartiges Verfahren geeignet sein.

Das Ziel des Maschenverfahrens besteht in der Verflechtung von Luft- und Druckbilanzen sowie anschließender Berechnung der einzelnen Luftmassenströme beim Durchströmen eines (mehrräumigen) Gebäudes. Für die Luftbilanz gilt, dass die Summe aller Zuluftmassenströme gleich der Summe aller Abluftmassenströme sein muss, d.h.

$$
\sum \dot{m}_{e i n}=\sum \dot{m}_{a u s}
$$

Zur Berechnung der Druckbilanz werden die Räume bzw. Verbindungen zwischen Gebäudeteilen als Knoten betrachtet, alle übrigen Luftwege (Öffnungen, Fugen etc.) als Strecken. Eine Strecke ist durch einen konstanten Luftmassenstrom und durch einen Druckabfall in Strömungsrichtung gekennzeichnet. Knoten weisen veränderliche Massenströme auf, haben aber konstante Druckverhältnisse. Die äußere Atmosphäre wird als druckverlustlose "Ringleitung" oder äußerer Knoten um das Gebäude aufgefasst. Eine Masche ist eine in sich geschlossene Reihung aufeinanderfolgender Strecken. Die Anzahl an Strecken ist gleich der Anzahl der zu bestimmenden Luftmassenströme [15].

Zwischen der Anzahl der Maschen $m a$, Knoten $k n$ und Strecken $r$ besteht eine Beziehung

$$
r=m a+k n-1
$$

Die Berechnung der Durchströmung eines Gebäudes erfolgt dann durch zwei Bilanzen.

Massenbilanz für jeden Knoten:

$$
\sum_{i} \dot{m}_{i}=0
$$

Druckbilanz für jede Masche:

$$
\sum_{j} \Delta p_{j}=0
$$

In allen Maschen ist eine gleiche Strömungsrichtung anzunehmen, die Maschenbilanzen werden über eine gemeinsame Öffnung aufgestellt. Durch Einsetzen der gemessenen Drücke — Druckdifferenz 
infolge der Windanströmung, Auftriebsdruckdifferenz infolge von Dichteunterschieden, Druckverluste infolge der Durchströmung von Öffnungen, Fugen, Kanälen und Schächten — in Gleichung 63 kann das aufgestellte Gleichungssystem mit den Unbekannten $\dot{m}_{i}$ nach der internen Druckverteilung aufgelöst und berechnet werden. 


\section{Methode der Kompartimentalisierung räumlicher Systeme}

\subsection{Definition und Anwendungsbereiche}

Der Begriff "Kompartiment" entstammt der Zellenlehre. Ausgangspunkt jeder Kompartimentalisierung ist die Definition des Systems. Als System kann ein Organ, ein Lebewesen, ein Gebäude, ein Ökosystem etc. betrachtet werden. Der Begriff "System" umfasst eine abgegrenzte, endliche Zahl an Subsystemen, welche definierte Zustandsvariablen aufweisen und durch eine Vielzahl an Relationen untereinander gekennzeichnet sind. Diese Subsysteme werden als Kompartimente bezeichnet. Ein Kompartiment ist dabei definiert als Menge eines Stoffes (z.B. Konzentration), die kinetisch als homogene und abgeschlossene Einheit zu betrachten ist [33].

Die Unterteilung eines Systems in Kompartimente wird bereits seit Jahrzehnten, überwiegend im medizinischen, biomedizinischen und pharmakologischen Bereich [10], angewendet. Die vorhandene Literatur hierzu ist sehr umfangreich. Beispielhaft sei auf WEI [71] verwiesen; er betrachtet einen Menschen als System. Der Körper wird in die Kompartimente Blut, schnell durchblutete Organe (Herz, Gehirn, Leber) und langsam durchblutete Organe (Haut, Muskeln, Gewebe) unterteilt. Dem Organismus werden ungefährliche Tracermoleküle injiziert, welche sich unterschiedlich schnell im System "Mensch" ausbreiten bzw. umwandeln. Anhand der Transportgeschwindigkeit des Tracers lässt sich auf die Verweilzeit des Tracers in den unterschiedlichen Kompartimenten schließen. Der Einfluss von Medikamenten, Drogen etc. auf den menschlichen Organismus bzw. spezifischer Organe kann hiermit abgeschätzt werden.

Das Abschätzen des Verbleibs von Substanzen in mikroskopischen Systemen kann ebenfalls in makroskopischen Systemen untersucht werden. Die physikalischen Gesetzmäßigkeiten sind identisch, allerdings bereitet die Abgrenzung großer Kompartimente Schwierigkeiten [72].

EBERHARDT ET AL. [16] untersuchten den Verbleib bzw. die Bedeutung von Cäsium in der arktischen Nahrungskette.

VArga ET AL. [69] brachten flüssigen Sauerstoff als Tracer in Flüsse ein. Ziel: Feststellung der Transportwege von eingeleiteten Chemikalien.

Ein ähnliches Ziel verfolgten LEGE ET AL. [39] in ihren Untersuchungen zur Ausbreitung von Mülldeponieabfällen ins Grundwasser.

LOGOFET [43] verwendete das Kompartimentalisierungsverfahren, um ein globales Carbonmodell bzw. eine globale Carbonbilanz der Erde zu erstellen.

Die Bedeutung des Eintrags von Pflanzenschutzmitteln in Ökosysteme wurde u.a. von NÖRTERSHEUSER [51] sowie PAUL UND JAHNS [53] untersucht. Kompartimentalisierungsverfahren spielen bislang vor allem im pflanzlichen Bereich der Landwirtschaft eine große Rolle. Ein generelles Modell 
für alle Pflanzenschutzmittel existiert nach [51] dennoch nicht. Es muss auf die speziellen Anforderungen (Abbau- und Verteilungsvorgänge) angepasst werden.

Werden zum Beispiel der Boden, die Biota, die Luft und das Wasser als makroskopische Kompartimente im System Umwelt angesehen, ist eine rein physikalische Abgrenzung nicht möglich. Die einzelnen Kompartimente lassen sich verbal zwar schnell definieren, jedoch sind die notwendigen Differenzierungen sowie die jeweils spezifischen Mechanismen für den Stoffaustausch schwer zu bestimmen [53]. Es ist nicht so, dass Boden, Biota, Luft und Wasser homogene Kompartimente darstellen, deren Zustandseigenschaften sich an den jeweiligen Kompartimentsgrenzen sprunghaft ändern. Vielmehr treten nichtlineare Zusammenhänge kompartimentsintern auf - eine wesentliche Eigenschaft von Ökosystemen [62].

Trotz der unbestreitbaren Nichtlinearität eines solch makroskopischen Systems muss diese überschaubar bleiben. Dazu werden Modelle verwendet, die die komplizierten Strukturen eines Systems vereinfachen, ohne die grundsätzlichen Zusammenhänge zu verändern. Das Kompartimentalisierungsverfahren ist ein solches Modell.

Wichtigste Annahme eines Kompartimentemodells ist die gleichmäßige Verteilung einer Substanz (Tracer) mit nur geringen örtlichen Abweichungen innerhalb eines jeden Kompartimentes [7]. Die Gleichverteilung bedeutet, dass die Änderung der Tracerkonzentration im Kompartiment schnell und gleichmäßig wirkt, zumindest schneller als ein Austausch über die Kompartimentsgrenzen hinweg. Die Konzentration im Kompartiment unterliegt sehr wohl zeitlichen Änderungen. Jede Konzentrationsänderung sollte aber so schnell stattfinden, dass die örtlichen Konzentrationsgradienten $(\operatorname{grad} C$ ) innerhalb eines Kompartimentes gegenüber den zeitlichen Gradienten des Austausches über die Kompartimentsgrenzen vernachlässigt werden können [33].

Die Unterteilung eines Systems in Kompartimente verfolgt demnach den Zweck, die Ortsabhängigkeit zu vernachlässigen und die Zeit als einzige unabhängige Variable zuzulassen (siehe auch Kapitel 4.4). Damit fließt in die Berechnung des Luftmassenstromes nur die zeitliche Änderung der Tracerkonzentration ein! Zur Zeitdiskretisierung, d.h. zur Diskretisierung des späteren Differentialgleichungssystems, können analoge Techniken wie für die Ortskoordinaten verwendet werden: Finite Differenzen, -Volumen, -Elemente Verfahren [39].

Eine weitere Annahme ist die Linearität der Übertragungsmechanismen. Linear bedeutet, dass der Stoffaustausch des Kompartimentes proportional zur Quellkonzentration im Kompartiment erfolgt [43]. Die Linearität bezieht sich dabei nur auf die Tracerkonzentration und nicht auf die die Austauschvorgänge beeinflussenden Parameter wie Temperatur oder Zeit. Ein Kompartiment fungiert einerseits als Senke getätigter Interaktionen, welche von benachbarten Kompartimenten ausgehen, andererseits als potentielle Quelle neuer Interaktionen [20].

Die Materialeigenschaften des eingebrachten Tracers werden durch die Transport- bzw. Austausch- 
koeffizienten $a_{j i}, a_{i j}$ beschrieben. In diesen Koeffizienten sind die von den verschiedenen Massenaustauschvorgängen (Konvektion, Diffusion) stammenden Anteile subsummiert. Ziel von Kompartimentalisierungsmodellen ist die Abschätzung bzw. Erfassung dieser Austauschkoeffizienten.

\subsection{Allgemeine Vorgehensweise}

Bei der Kompartimentalisierung werden Input- und Outputvorgänge einzelner Kompartimente betrachtet. Die mathematische Grundlage zur Untersuchung des Verbleibs eines Tracers in einem System sind die Massenbilanzen des Tracers in den einzelnen Kompartimenten. In eine Massenbilanz müssen alle relevanten Stoffbewegungen einfließen: Konvektion $K$, Diffusion $D$, Quellen $Q$, Senken $S$ und Umwandlungsvorgänge $U$.

$$
\dot{m}_{\text {Tracer }}=K+D+Q+S+U
$$

Für jedes Kompartiment wird eine eigene Bilanzgleichung erstellt. Diese beschreibt die Änderung der Tracerkonzentration im Kompartiment im Verhältnis zum Traceraustausch mit den benachbarten Kompartimenten. Betrachtet man Abbildung 18, so gilt für jedes Kompartiment $i$ folgende Massenbilanz aus Eintrag $e_{i}$, Austausch $a_{j i}, a_{i j}$ und Abbau $a_{i 0}$ :

$$
\dot{m}_{i}=e_{i}+\sum_{\substack{j=1 \\ j \neq i}}^{n} C_{j} \cdot a_{j i}-\sum_{\substack{i=1 \\ i \neq j}}^{n} C_{i} \cdot a_{i j}-C_{i} \cdot a_{i 0}
$$

Aufgrund der besseren Übersichtlichkeit werden Systeme linearer Gleichungen (Gl. 66) in Form von Matrizen aufgestellt. Die Schreibweise für ein lineares Gleichungssystem in Matrixform lautet [75]:

$$
M \cdot x=b
$$

$M$ stellt die Matrix der Austauschkoeffizienten dar $\left(a_{j i}, a_{i j}\right), x$ den Lösungsvektor $\left(C_{i}, C_{j}\right)$, den es zu bestimmen gilt, und $b$ den Ergebnisvektor $\dot{m}_{i}$. Bei Kenntnis der Koeffizientenmatrix besitzt das Gleichungssystem stets eine quadratische Form und lässt sich entsprechend lösen.

\section{System "Nutztierstall”}

Der von JANSSEN UND KRAUSE [32] verfolgte Modellansatz kommt der eigenen Zielvorstellung recht nahe. Es wird versucht, die Wechselwirkungen und Abhängigkeiten zwischen emissionsbestimmenden Faktoren zu bestimmen. Das System “Tierstall” wird dazu in die Subsysteme Luft, Fußboden, 


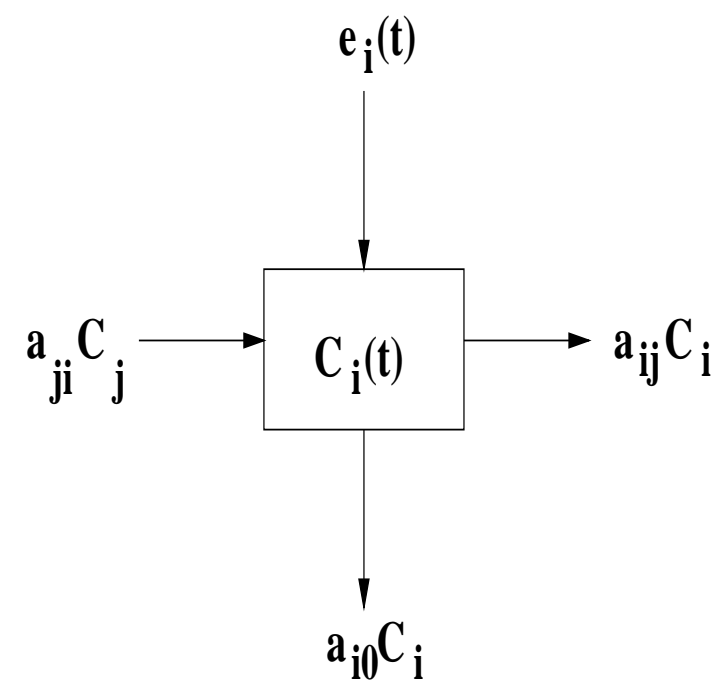

Abbildung 18: Stoffströme durch ein Kompartiment.

Gülle und Tier unterteilt (Abb. 19). Mit der Luft steht ein universelles Transportmedium zur Verfügung, es bestehen zusätzliche Austauschmöglichkeiten zwischen den anderen Kompartimenten. Die erforderliche Koeffizientenmatrix $M$ hat demnach die Form

$$
M=\left[\begin{array}{cccc}
a_{11} & 0 & a_{31} & a_{41} \\
a_{12} & a_{22} & a_{32} & a_{42} \\
a_{13} & 0 & a_{33} & a_{43} \\
a_{14} & a_{24} & a_{34} & a_{44}
\end{array}\right]
$$

Durch die Position der Koeffizienten in der Matrix ist die Höhe und die Richtung des Massenaustausches zwischen benachbarten Kompartimenten eindeutig festgelegt. Austauschkoeffizienten sind stets größer oder gleich Null. Für die Hauptdiagonalelemente $a_{i i}$ gilt, dass sie gleich der negativen Summe aller Spaltenelemente minus der Rate des Abbaus $a_{i 0}$ sind. Sie haben keinerlei Aussagekraft hinsichtlich der Beschreibung der Transportvorgänge, sondern sind lediglich für die Berechnung des Gleichungssystems erforderlich:

$$
a_{i i}=-\sum_{\substack{i=1 \\ i \neq j}}^{n} a_{i j}-a_{i 0}
$$

Der erste Index eines Austauschkoeffizientens steht für das "gebende" Kompartiment, der zweite Index kennzeichnet das "nehmende" Kompartiment. Im gezeigten Beispiel werden die Austauschkoeffizienten so gewählt bzw. abgeschätzt, dass sich in einer vorgegebenen Zeit eine bestimmte Konzentration im System einstellt. 


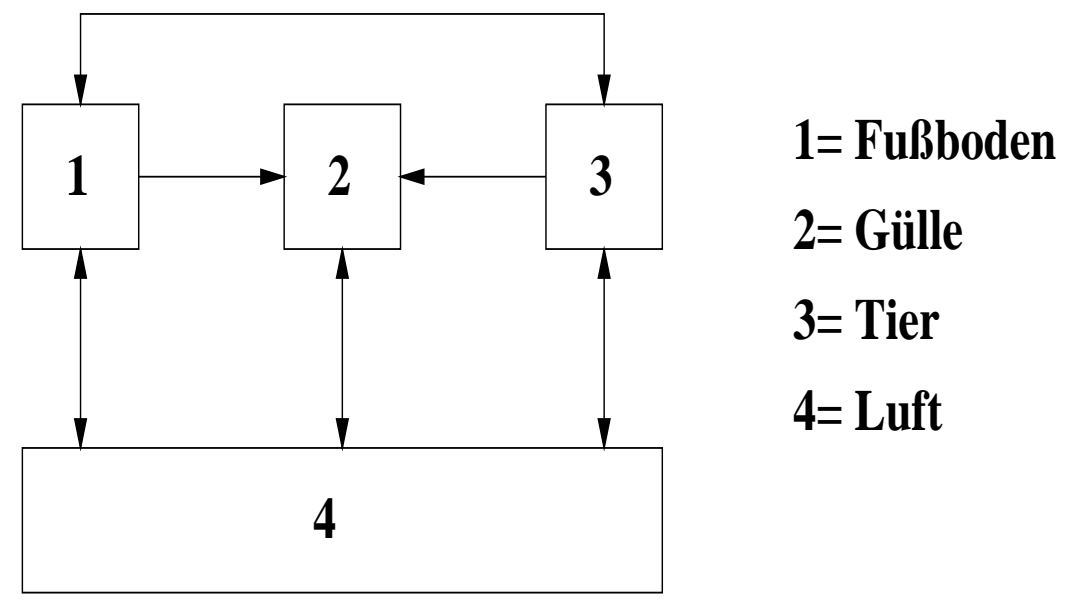

Abbildung 19: Aufteilung des Systems Nutztierstall in vier Teilbereiche (nach [32]).

Die Struktur der Matrix gibt somit die prinzipiellen Stoffaustauschvorgänge wieder. Sonderfälle von Systemen, wie sie sehr zahlreich und anschaulich in [33] beschrieben werden, können damit ebenso abgehandelt werden wie "einfache" Systeme.

Im Folgenden wird die allgemeine Vorgehensweise bei der Kompartimentalisierung anhand eines ausgewählten Beispiels aufgezeigt.

\subsection{Entwicklung der Systemgleichungen}

Angenommen wird zunächst ein System ohne jegliche Begrenzung; ein ungehinderter Austausch von Luft und Tracer kann in alle Richtungen erfolgen.

Unter Anwendung der Kompartimentalisierung in Form eines Finite Volumen Verfahrens erfolgt die Diskretisierung des Raumes in (gleichgroße) makroskopische Teilbereiche (Abb. 20 untere Zeichnung). Ausgehend von einer wahllosen Anordnung der Konzentrationsbereiche im Raum wird das System fiktiv aufgeteilt. Es wird angenommen, dass die Konzentrationsverteilung im Gesamtsystem inhomogen, innerhalb eines jeden Teilbereiches jedoch homogen ist. Im Unterschied zur Einteilung eines z.B. heterogenen Systems (Umwelt) in homogene Kompartimente (Luft, Boden, Biota, Wasser) wie im vorherigen Abschnitt, wird in dieser Arbeit das Ziel verfolgt, die Ausbreitungsvorgänge eines Tracers in der Luft zu beschreiben. Die Luft wird wiederum als System verstanden, welches in makroskopische "Luftpakete" unterteilt ist. In jedes Luft-Kompartiment wird ein Tracer schlagartig eingebracht. Quellen sind danach nicht vorhanden. Des weiteren können Senken und Umwandlungsvorgänge durch eine enge zeitliche Messwerteerfassung vernachlässigt werden. Unter Annahme von $D=0$ (Kapitel 3, Abschnitt 3.2.2) reduziert sich Gleichung 65 auf den konvektiven Stofftransport. 

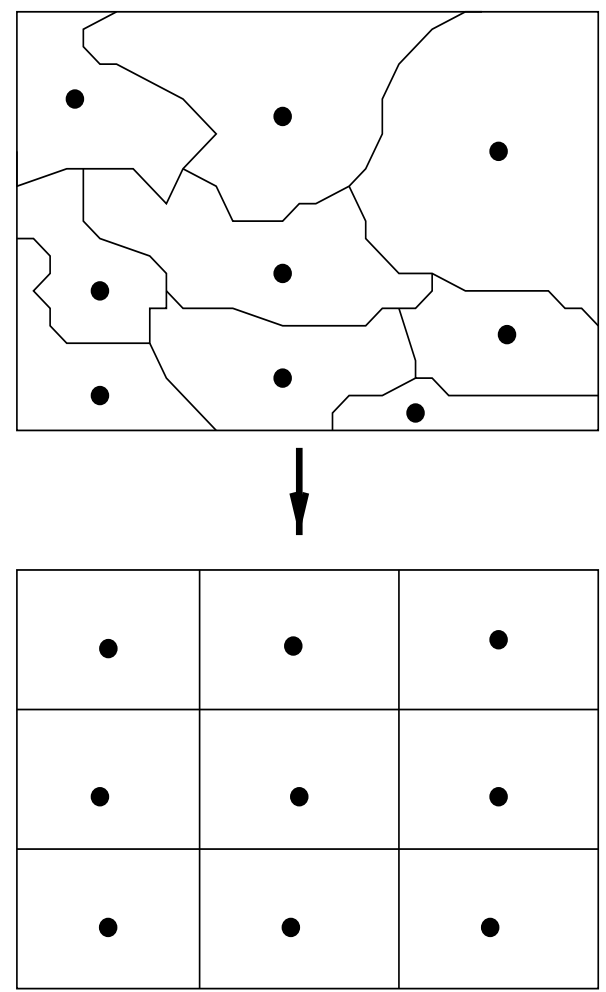

Abbildung 20: Idealisierter Übergang von ungeordneter Raumstruktur zu definierten Kompartimentvolumina.

Einen schematischen Überblick über die Anordnung der Kompartimente sowie alle möglichen Austauschbeziehungen für das gewählte theoretische Beispiel vermittelt Abbildung 21.

Im Zentrum eines jeden Kompartimentes befindet sich der zu diesem Kompartiment zugehörige Messsensor. In jedem Kompartiment wird das Abklingen der Tracerkonzentration mit der Zeit festgehalten. Die Konzentration in einem Kompartiment und deren zeitliche Änderung stellen die einzigen verfügbaren Informationen und damit quasi eine Art "Fingerabdruck" des Systems dar. Aufgrund des spezifischen Strömungsverlaufs, wie in Abbildung 4 ersichtlich, resultiert an jedem dieser Sensoren eine Abklingfunktion unterschiedlicher Steigung und damit als Indiz für einen unterschiedlichen Luftaustausch. Ein Austausch an Luft bzw. Tracergas kann nur über die Kompartimentsgrenzen erfolgen. Im zweidimensionalen Fall sind dies vier, im dreidimensionalen Fall sechs Kompartimentsgrenzen.

Ziel ist es, anhand des Abklingverhaltens mit der Zeit und unter Berücksichtigung der Massenerhaltung den Volumenstrom zu berechnen. Weiterhin soll geprüft werden, inwieweit unter Anwendung der Kompartimentalisierung repräsentative Ergebnisse erzielt bzw. diese auf Praxisanlagen übertragen werden können. 
Austausch zwischen Stall- und Umgebungskompartiment

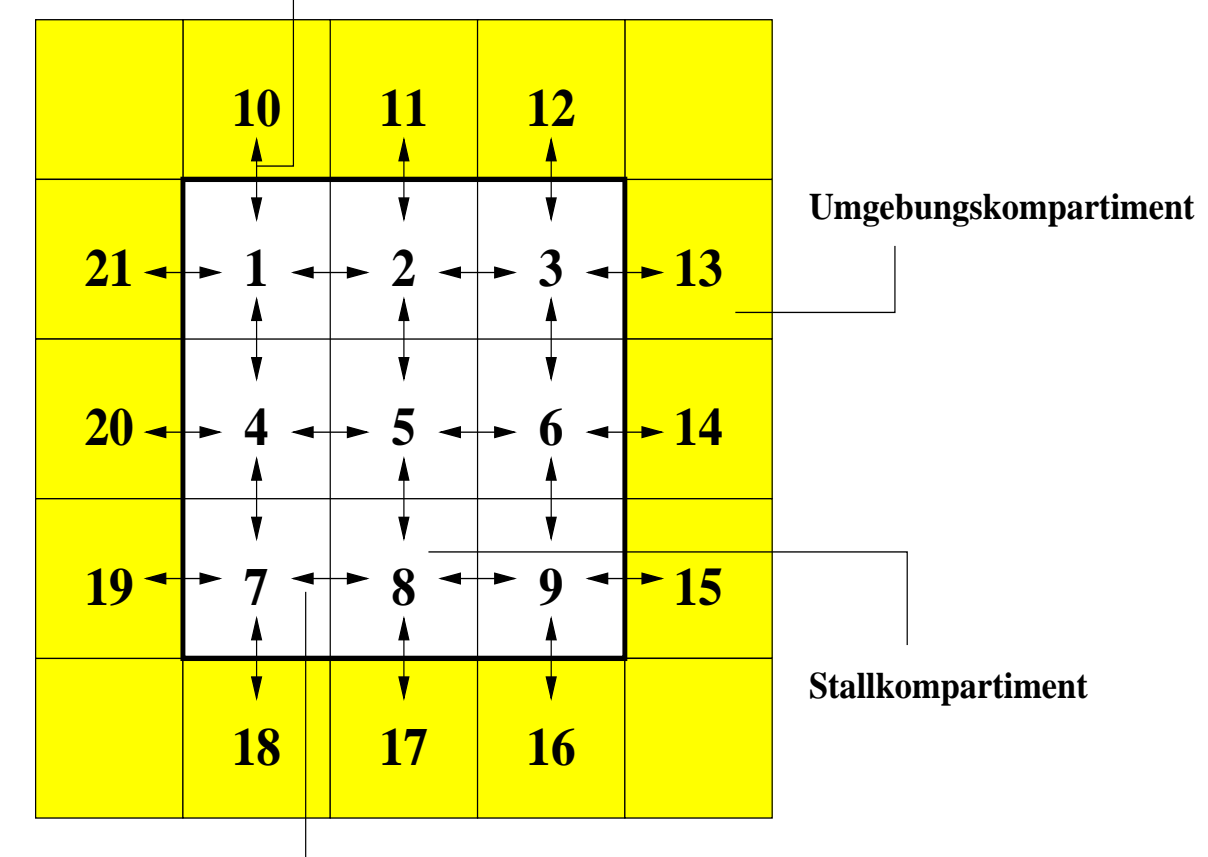

Austausch zwischen Stallkompartimenten

Abbildung 21: Anzahl, Anordnung und Austauschmöglichkeiten der Stall- und Umgebungskompartimente.

\subsubsection{Herleitung des linearen Gleichungssystems}

Die Konzentration des in ein System eingebrachten Tracers variiert. Die Erhaltungsbedingung für die Tracermasse ist jedoch zu erfüllen. Das bedeutet, die Tracermasse, welche einem Kompartiment zugeführt wird, muss dieses Kompartiment auch verlassen, wenn Quellen, Senken oder Umwandlungsvorgänge des Tracers ausgeschlossen werden. Der Massenaustausch eines Kompartimentes ist demnach definiert als

$$
\begin{aligned}
\dot{m}_{\text {Tracer }} & =\dot{m}_{\text {ein }}-\dot{m}_{\text {aus }} \\
\frac{\partial C}{\partial t} \cdot V & =(C \cdot \dot{V})_{e i n}-(C \cdot \dot{V})_{\text {aus }}
\end{aligned}
$$

Betrachtet man die Produkte $(C \cdot \dot{V})_{e i n}$ und $(C \cdot \dot{V})_{a u s}$ als Austausch, lässt sich Gleichung 69 auch wie folgt schreiben

$$
\frac{\partial C}{\partial t} \cdot V=A T C_{e i n}-A T C_{a u s}
$$




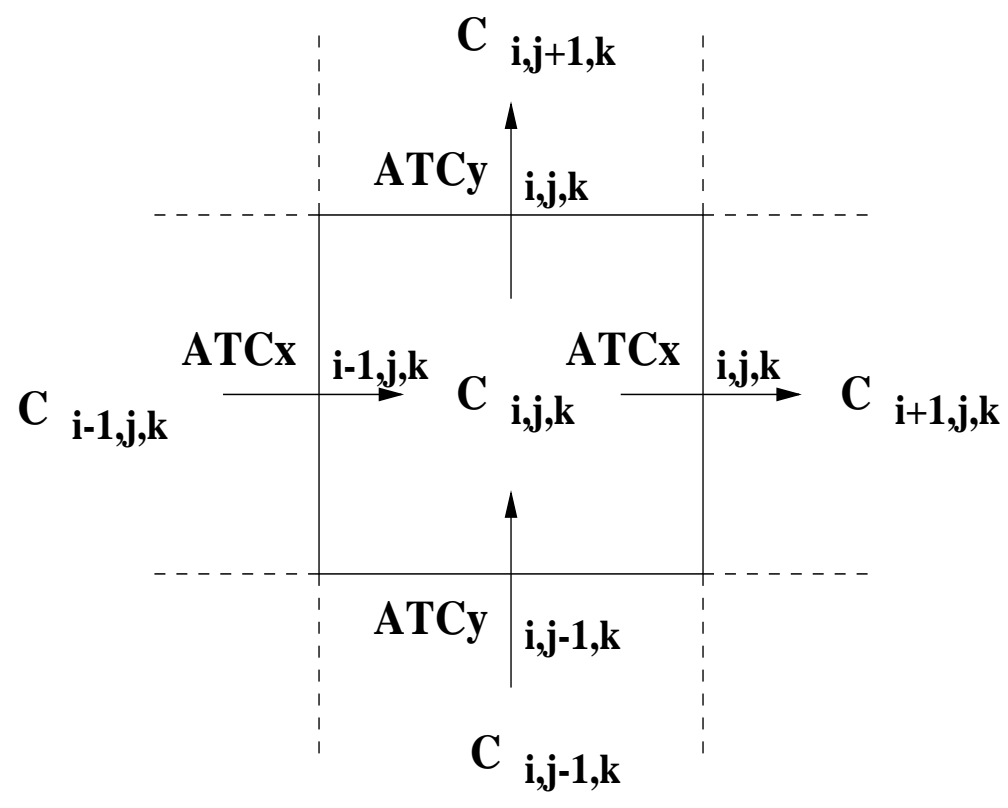

Abbildung 22: Austauschgrößen der Konzentration (ATC) beim Übergang zwischen Kompartimenten. $\mathrm{k}$ ist als Index für die dritte Dimension anzusehen.

$A T C$ steht für den Austausch von C. Dieser erfolgt mittig über die Kompartimentsgrenzen (Abb. 22). Die Konzentration ist ein Skalar; Skalare wirken von der Zellmitte aus. Das Geschwindigkeitsfeld $\vec{v}=\vec{v}(u, v, w)$ ist ein Vektor mit den Komponenten $u, v$ und $w$ in Richtung der Koordinatenachsen $x, y$ und $z$. Weiterhin gilt $V=$ konstant.

Stoffaustauschvorgänge beruhen auf der zeitlichen Änderung der Stoffkonzentration. Stellt $n$ den Zeitschritt dar, so ergibt sich in Anlehnung an Gleichung 70 die Bilanzgleichung für das betrachtete Volumenelement $V_{i, j, k}$ (=Kompartiment) mit der Konzentration $C_{i, j, k}$ aus Abbildung $22 \mathrm{zu}$ :

$$
\frac{C_{i, j, k}^{n+1}-C_{i, j, k}^{n}}{t^{n+1}-t^{n}} \cdot V_{i, j, k}=A T C x_{i-1, j, k}^{n}+A T C y_{i, j-1, k}^{n}-A T C x_{i, j, k}^{n}-A T C y_{i, j, k}^{n}
$$

Abbildung 22 stellt eine vereinfachte zweidimensionale Darstellung dar, die dritte Dimension ist als unendliche Tiefe anzusehen. Mit Blick auf Abbildung 23 lässt sich der Austausch von $C$ über eine fiktive Kompartimentsgrenzfläche z.B. für $A T C x_{i, j, k}^{n}$ über die folgende Austauschbeziehung bestimmen:

$$
\begin{aligned}
Z D & =\text { Zellendifferenz } \\
Z D & =(1-l x) \Delta x_{i}+(1+l x) \Delta x_{i+1} \\
A T C x_{i, j, k}^{n} \cdot Z D & =A x_{i, j, k} \cdot u_{i, j, k}^{n+1} \cdot\left[(1-l x) \Delta x_{i} \cdot C_{i+1, j, k}^{n}+(1+l x) \Delta x_{i+1} \cdot C_{i, j, k}^{n}\right]
\end{aligned}
$$




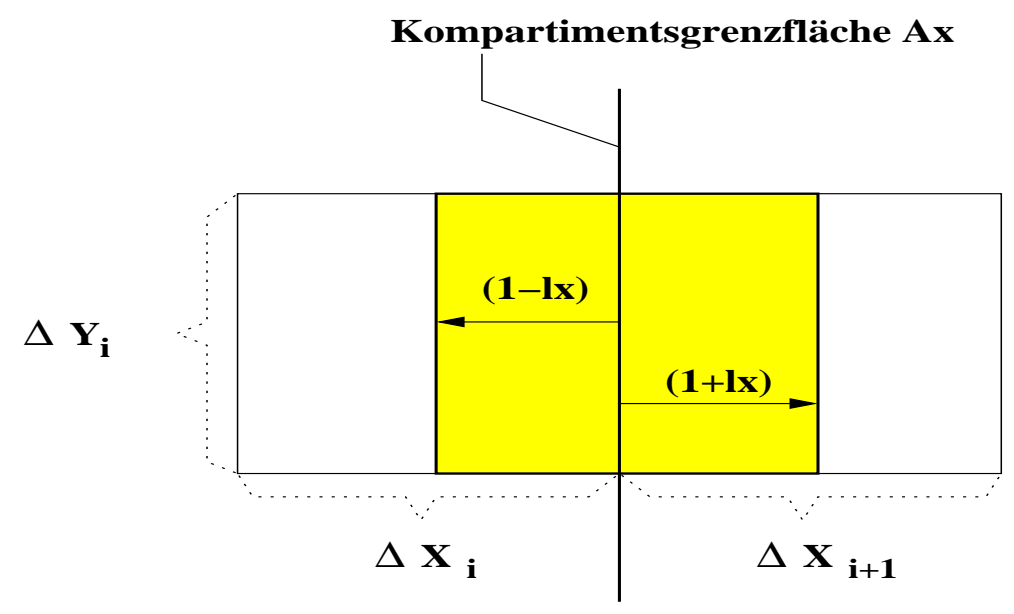

Abbildung 23: Austausch eines Skalars über eine Grenzfläche. Die Zellabmessungen des betrachteten Kompartimentes sind $\Delta x_{i}$ und $\Delta y_{i}$.

$$
A T C x_{i, j, k}^{n}=A x_{i, j, k} \cdot u_{i, j, k}^{n+1} \cdot \frac{(1-l x) \Delta x_{i} \cdot C_{i+1, j, k}^{n}+(1+l x) \Delta x_{i+1} \cdot C_{i, j, k}^{n}}{Z D}
$$

Für $\Delta x_{i}=\Delta x_{i+1}$ vereinfacht sich die Beziehung zu Gleichung 74

$$
A T C x_{i, j, k}=A x_{i, j, k} \cdot u \cdot \frac{(1+\operatorname{sign}(n)) \cdot C_{i, j, k}+(1-\operatorname{sign}(n)) \cdot C_{i+1, j, k}}{2}
$$

Im Regelfall gilt aber $\Delta x_{i} \neq \Delta x_{i+1}$.

$l x$ ist eine dimensionslose fiktive Größe, die den Betrag der Geschwindigkeit $v$ (mit der die Konzentration über die Grenzfläche strömt) sowie die Richtung der Geschwindigkeit wiedergibt [38]:

$$
l x=v \cdot \operatorname{sign}\left(u_{i, j, k}^{n}\right) \quad ; v \leq 1
$$

A priori besteht keine Information über $u_{i, j, k}^{n}$, so dass $v=0$ gesetzt werden kann. Gleiches gilt analog für $l y$ und $l z$, die die Größen der Geschwindigkeit für die y- und z-Richtung beinhalten.

Unter Zugrundelegung der Kontinuitätsgleichung für den Tracer (G1. 52) ergibt sich aus Gleichung 73 die vollständige Massenbilanz

$$
\begin{aligned}
\frac{C_{i, j, k}^{n+1}-C_{i, j, k}^{n}}{t^{n+1}-t^{n}} \cdot V_{i, j, k}= & A x_{i-1, j, k} \cdot u_{i-1, j, k}^{n+1} \cdot \frac{(1-l x) \Delta x_{i} \cdot C_{i-1, j, k}^{n}+(1+l x) \Delta x_{i-1} \cdot C_{i, j, k}^{n}}{(1-l x) \Delta x_{i}+(1+l x) \Delta x_{i-1}} \\
& -A x_{i, j, k} \cdot u_{i, j, k}^{n+1} \cdot \frac{(1-l x) \Delta x_{i} \cdot C_{i+1, j, k}^{n}+(1+l x) \Delta x_{i+1} \cdot C_{i, j, k}^{n}}{(1-l x) \Delta x_{i}+(1+l x) \Delta x_{i+1}} \\
& +A y_{i, j-1, k} \cdot v_{i, j-1, k}^{n+1} \cdot \frac{(1-l y) \Delta y_{i} \cdot C_{i, j-1, k}^{n}+(1+l y) \Delta y_{i-1} \cdot C_{i, j, k}^{n}}{(1-l y) \Delta y_{i}+(1+l y) \Delta y_{i-1}}
\end{aligned}
$$




$$
\begin{aligned}
& -A y_{i, j, k} \cdot v_{i, j, k}^{n+1} \cdot \frac{(1-l y) \Delta y_{i} \cdot C_{i, j+1, k}^{n}+(1+l y) \Delta y_{i+1} \cdot C_{i, j, k}^{n}}{(1-l y) \Delta y_{i}+(1+l y) \Delta y_{i+1}} \\
& +A z_{i, j, k-1} \cdot w_{i, j, k-1}^{n+1} \cdot \frac{(1-l z) \Delta z_{i} \cdot C_{i, j, k-1}^{n}+(1+l z) \Delta z_{i-1} \cdot C_{i, j, k}^{n}}{(1-l z) \Delta z_{i}+(1+l z) \Delta z_{i-1}} \\
& -A z_{i, j, k} \cdot w_{i, j, k}^{n+1} \cdot \frac{(1-l z) \Delta z_{i} \cdot C_{i, j, k+1}^{n}+(1+l z) \Delta z_{i+1} \cdot C_{i, j, k}^{n}}{(1-l z) \Delta z_{i}+(1+l z) \Delta z_{i+1}}
\end{aligned}
$$

Wegen $v=0$ fallen die Terme $l x, l y$, und $l z$ heraus. Durch einen Koeffizientenvergleich werden die Grenzflächen ( $A x, A y, A z)$ und die Geschwindigkeiten $(u, v, w)$ zu einer unbekannten Größe zusammengefasst: Austauschkoeffizient a. a weist die Dimension 'Länge ${ }^{3} / Z e i t$ ' auf und damit die Dimension eines Volumenstromes. Das Produkt $a \cdot C$ gibt dann den Massenfluss über die jeweilige Grenzfläche wieder. Das Gleichungssystem 76 kann vereinfachend geschrieben werden

$$
\begin{aligned}
\frac{C_{i, j, k}^{n+1}-C_{i, j, k}^{n}}{t^{n+1}-t^{n}} \cdot V_{i, j, k}= & a x_{i-1, i} \cdot C_{i-1, j, k}^{n}-a x_{i, i-1} \cdot C_{i, j, k}^{n} \\
& +a x_{i+1, i} \cdot C_{i+1, j, k}^{n}-a x_{i, i+1} \cdot C_{i, j, k}^{n} \\
& +a y_{j-1, j} \cdot C_{i, j-1, k}^{n}-a y_{j, j-1} \cdot C_{i, j, k}^{n} \\
& +a y_{j+1, j} \cdot C_{i, j+1, k}^{n}-a y_{j, j+1} \cdot C_{i, j, k}^{n} \\
& +a z_{k-1, k} \cdot C_{i, j, k-1}^{n}-a z_{k, k-1} \cdot C_{i, j, k}^{n} \\
& +a z_{k+1, k} \cdot C_{i, j, k+1}^{n}-a z_{k, k+1} \cdot C_{i, j, k}^{n}
\end{aligned}
$$

Mit negativem Vorzeichen versehene Flussraten stehen für Austauschkoeffizienten aus dem betrachteten Kompartiment $V_{i, j, k}$. Die mit positivem Vorzeichen versehenen Flussraten gehören jeweils zu einem der umgebenden Kompartimente und zwar:

$V_{i-1, j, k} ; V_{i+1, j, k} ; V_{i, j-1, k} ; V_{i, j+1, k} ; V_{i, j, k-1} ; V_{i, j, k+1}$

Der erste Index von $a$ symbolisiert das "gebende" Kompartiment, der zweite Index das "nehmende" Kompartiment. In Abbildung 24 sind die möglichen Austauschkoeffizienten dargestellt.

Im einfachsten Fall grenzen an ein quaderförmiges Kompartiment bei Kantendeckung sechs weitere quaderförmige Kompartimente. Das heißt, sechs Austauschkoeffizienten des Kompartimentes $V_{i, j, k}$ sind von "innen" nach "außen" wirksam und jeweils eines von den umgebenden Kompartimenten auf $V_{i, j, k}$. Mit Hilfe der gemessenen Zeitreihen der Konzentration $C$ in den jeweiligen Kompartimenten lässt sich ein gekoppeltes Gleichungssystem aufbauen. Das Gleichungssystem besitzt demnach folgenden Aufbau:

$$
V_{i} \cdot \frac{d C_{i}}{d t}=\sum_{\substack{j=1 \\ j \neq i}}^{n} C_{j} \cdot a_{j i}-\sum_{\substack{i=1 \\ i \neq j}}^{n} C_{i} \cdot a_{i j}
$$




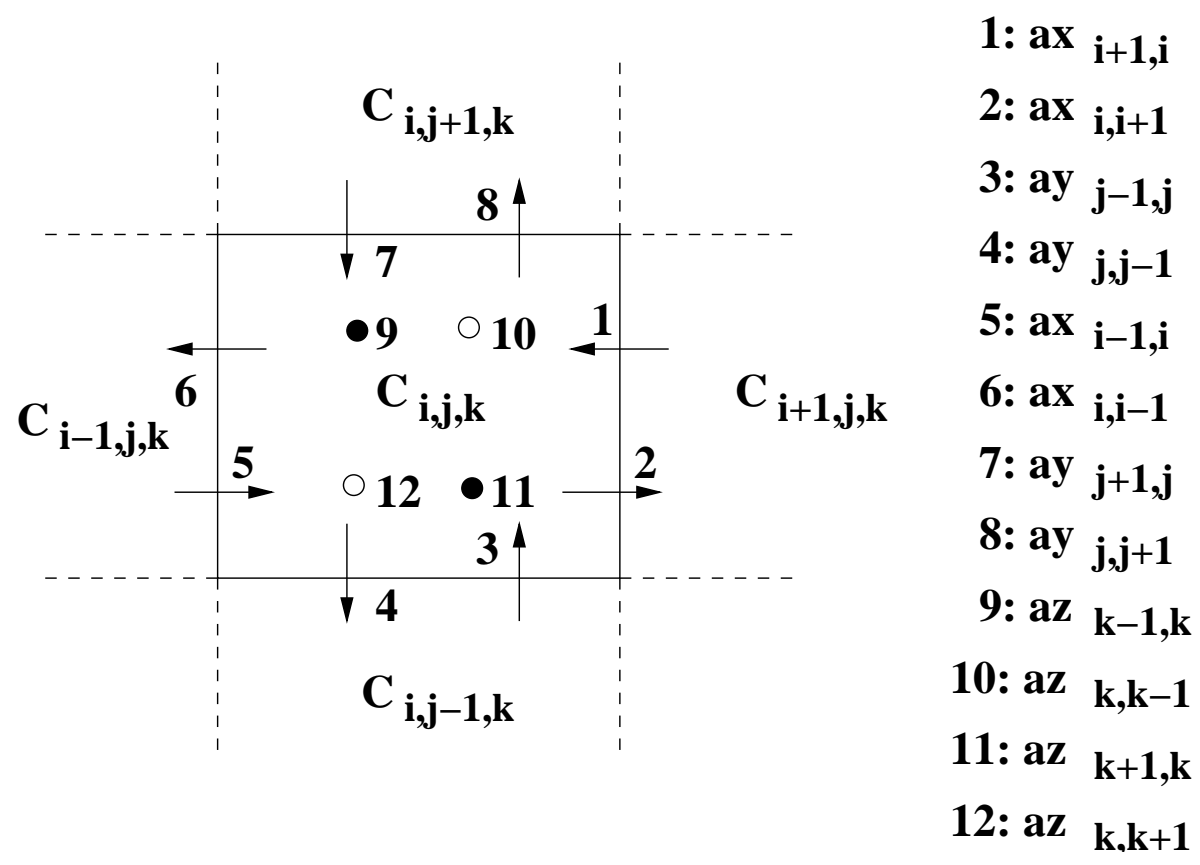

Abbildung 24: Mögliche Austauschkoeffizienten eines quaderförmigen Kompartimentes, hier vereinfachend zweidimensional dargestellt.

Für das in Abbildung 4 betrachtete Simulationsbeispiel lautet das vollständige Gleichungssystem unter Verwendung der Kompartimentanordnung aus Abbildung 21:

$$
\begin{aligned}
V_{1} \cdot \frac{d C_{1}}{d t=} & C_{2} \cdot a_{21}+C_{4} \cdot a_{41}+C_{10} \cdot a_{101}+C_{21} \cdot a_{211} \\
& -C_{1} \cdot\left(a_{12}+a_{14}+a_{110}+a_{121}\right) \\
V_{2} \cdot \frac{d C_{2}}{d t}= & C_{1} \cdot a_{12}+C_{3} \cdot a_{32}+C_{5} \cdot a_{52}+C_{11} \cdot a_{112} \\
& -C_{2} \cdot\left(a_{21}+a_{23}+a_{25}+a_{211}\right) \\
V_{3} \cdot \frac{d C_{3}}{d t}= & C_{2} \cdot a_{23}+C_{6} \cdot a_{63}+C_{12} \cdot a_{123}+C_{13} \cdot a_{133} \\
& -C_{3} \cdot\left(a_{32}+a_{36}+a_{312}+a_{313}\right) \\
V_{4} \cdot \frac{d C_{4}}{d t}= & C_{1} \cdot a_{14}+C_{5} \cdot a_{54}+C_{7} \cdot a_{74}+C_{20} \cdot a_{204} \\
& -C_{4} \cdot\left(a_{41}+a_{45}+a_{47}+a_{420}\right) \\
V_{5} \cdot \frac{d C_{5}}{d t}= & C_{2} \cdot a_{25}+C_{4} \cdot a_{45}+C_{6} \cdot a_{65}+C_{8} \cdot a_{85} \\
& -C_{5} \cdot\left(a_{52}+a_{54}+a_{56}+a_{58}\right) \\
V_{6} \cdot \frac{d C_{6}}{d t}= & C_{3} \cdot a_{36}+C_{5} \cdot a_{56}+C_{9} \cdot a_{96}+C_{14} \cdot a_{146} \\
& -C_{6} \cdot\left(a_{63}+a_{65}+a_{69}+a_{614}\right) \\
V_{7} \cdot \frac{d C_{7}}{d t}= & C_{4} \cdot a_{47}+C_{8} \cdot a_{87}+C_{18} \cdot a_{187}+C_{19} \cdot a_{197}
\end{aligned}
$$




$$
\begin{aligned}
& -C_{7} \cdot\left(a_{74}+a_{78}+a_{718}+a_{719}\right) \\
V_{8} \cdot \frac{d C_{8}}{d t}= & C_{5} \cdot a_{58}+C_{7} \cdot a_{78}+C_{9} \cdot a_{98}+C_{17} \cdot a_{178} \\
& -C_{8} \cdot\left(a_{85}+a_{87}+a_{89}+a_{817}\right) \\
V_{9} \cdot \frac{d C_{9}}{d t}= & C_{6} \cdot a_{69}+C_{8} \cdot a_{89}+C_{15} \cdot a_{159}+C_{16} \cdot a_{169} \\
& -C_{9} \cdot\left(a_{96}+a_{98}+a_{915}+a_{916}\right)
\end{aligned}
$$

Die jeweiligen Massenbilanzgleichungen drücken aus, dass innerhalb eines Zeitschrittes nur soviel Tracermasse aus einem Kompartiment exportiert werden kann, wie es gleichzeitig an Tracermassenimport erfahren kann.

\subsubsection{Endgültiges Gleichungssystem}

Dem aufgestellten Gleichungssystem 79 liegt die Annahme eines ungestörten Massenaustausches in alle Raumrichtungen zugrunde. Eine mathematisch-numerische Fragestellung wird im Allgemeinen gegliedert in ein Gleichungssystem und in ein System von "Randbedingungen".

Betrachtet man ein Gesamtsystem, z.B. einen Stall, so stellen die Stallabmessungen (Länge, Breite, Höhe) die physikalischen Begrenzungen dar. Dabei muss zwischen undurchlässigen und durchlässigen Begrenzungen unterschieden werden. Stößt ein Kompartiment an eine Wand, so ist der Austauschkoeffizient vom Kompartiment in Richtung Umwelt gleich Null. Stößt ein Kompartiment hingegen an eine Öffnung, dann existiert jeweils ein Austauschkoeffizient vom Kompartiment in Richtung Umwelt und von der Umwelt in Richtung Kompartiment. Letzterer Massenfluss ist allerdings nur für die Luft möglich, da kein Tracer von außen in das System gelangen kann. Das Gleichungssystem 79 ist somit um die "Randbedingungen" zu erweitern, d.h. alle nicht existenten Austauschkoeffizienten werden gleich Null gesetzt.

Zusätzlich muss das Gleichungssystem 79 um das Korrektiv div $\vec{v}=0$ erweitert werden. Es beruht auf der Kontinuitätsgleichung für das Trägermedium Luft (Gl. 35) und besagt, dass der Betrag der eintretenden Luftmenge in das System gleich dem Betrag der austretenden Luftmenge aus dem System sein muss. Da die Dichte der Luft als konstant angenommen wird, muss die LuftKontinuitätsbedingung deshalb nicht für jedes Kompartiment hinzugefügt werden. Es genügt die Gesamtkontinuitätsgleichung (Gl. 80) für das System in der Form

$$
0=\sum_{\substack{i=1 \\ j>n}}^{n} a_{j i}-\sum_{\substack{i=1 \\ j>n}}^{n} a_{i j}
$$


Die Umwelt wird in diesem Zusammenhang als Null "0" bezeichnet. Im betrachteten Simulationsbeispiel sind das dann die Austauschkoeffizienten $a_{01}$ und $a_{90}$. Aus dem Gleichungssystem 79 ergibt sich das endgültige Gleichungssystem $81 \mathrm{zu}$ :

$$
\begin{aligned}
& V_{1} \cdot \frac{d C_{1}}{d t}=C_{2} \cdot a_{21}+C_{4} \cdot a_{41}-C_{1} \cdot\left(a_{10}+a_{12}+a_{14}\right) \\
& V_{2} \cdot \frac{d C_{2}}{d t}=C_{1} \cdot a_{12}+C_{3} \cdot a_{32}+C_{5} \cdot a_{52}-C_{2} \cdot\left(a_{21}+a_{23}+a_{25}\right) \\
& V_{3} \cdot \frac{d C_{3}}{d t}=C_{2} \cdot a_{23}+C_{6} \cdot a_{63}-C_{3} \cdot\left(a_{32}+a_{36}\right) \\
& V_{4} \cdot \frac{d C_{4}}{d t}=C_{1} \cdot a_{14}+C_{5} \cdot a_{54}+C_{7} \cdot a_{74}-C_{4} \cdot\left(a_{41}+a_{45}+a_{47}\right) \\
& V_{5} \cdot \frac{d C_{5}}{d t}=C_{2} \cdot a_{25}+C_{4} \cdot a_{45}+C_{6} \cdot a_{65}+C_{8} \cdot a_{85} \\
& V_{6} \cdot \frac{d C_{6}}{d t}=C_{5} \cdot\left(a_{52}+a_{54}+a_{56}+a_{58}\right) \\
& V_{7} \cdot \frac{d C_{7}}{d t}=C_{3} \cdot a_{36}+C_{5} \cdot a_{56}+C_{9} \cdot a_{96}-C_{6} \cdot\left(a_{63}+a_{65}+a_{69}\right) \\
& V_{8} \cdot \frac{d C_{8}}{d t}=C_{57} \cdot C_{7} \cdot\left(a_{74}+a_{78}\right) \\
& V_{9} \cdot \frac{d C_{9}}{d t}=C_{7} \cdot a_{78}+C_{9} \cdot a_{98}-C_{8} \cdot\left(a_{85}+a_{87}+a_{89}\right) \\
& 0=C_{8} \cdot a_{89}-C_{9} \cdot\left(a_{90}+a_{96}+a_{98}\right)
\end{aligned}
$$

Unter Zugrundelegung der allgemeinen Form der Matrizenschreibweise (Gl. 67, Kapitel 4.1)

$$
M \cdot x=b
$$

stellt $M$ die Matrix aller Austauschkoeffizienten dar $\left(\mathrm{a}_{i j}, \mathrm{a}_{j i}\right), x$ den Lösungsvektor $\left(\mathrm{C}_{i}\right)$, den es zu bestimmen gilt und $b$ den Ergebnisvektor $\left(V_{i} \cdot \frac{d C_{i}}{d t}\right)$. Bezieht man diese Betrachtungsweise auf das Vergleichsbeispiel, so ergibt sich folgendes Bild:

$$
\begin{aligned}
M & =\text { Koeffizientenmatrix (unbekannt) } \\
x & =\text { Lösungsvektor (bekannt) } \\
b & =\text { Ergebnisvektor (bekannt) }
\end{aligned}
$$

Wie zu erkennen ist, tritt im vorliegenden Fall ein inverses Problem auf: der eigentlich gesuchte Lösungsvektor $x$ ist bekannt, die Koeffizientenmatrix $M$ hingegen unbekannt. Beim Einsatz der 
Kompartimentalisierungsmethode ist also die Lösung in Form der aufgezeichneten Konzentrationsmessreihen des Tracers bereits vorher bekannt. Es gilt nun rückwirkend die Strömungsvorgänge in Form der Austauschkoeffizienten zu bestimmen, die das jeweilige Abklingverhalten an den Sensoren verursacht haben.

Um das aufgestellte Gleichungssystem zu lösen, gibt es zwei Möglichkeiten: Schätzung der Austauschkoeffizienten oder Umstellung des Gleichungssystems.

\section{Schätzung der Austauschkoeffizienten}

Das ursprünglich aufgestellte Gleichungssystem bleibt erhalten. Für die unbekannten Koeffizienten müssen Startwerte vorgegeben werden. Aus mathematischer Sicht existieren unendlich viele Möglichkeiten eines Startwertes. Anschließend würde mit Hilfe von Verfahren zur Parameteridentifikation solange optimiert, bis die errechneten Koeffizienten multipliziert mit den gemessenen Konzentrationswerten die reale Konzentrationsänderung in den jeweiligen Kompartimenten genau bzw. abzüglich einer bestimmten Fehlertoleranz wiedergäben (siehe Abschnitt 4.2). Da das vorliegende lineare Gleichungssystem ein gekoppeltes Gleichungssystem darstellt, haben Änderungen in den Austauschkoeffizienten Veränderungen im Gleichungssystem zur Folge. Der Zeitbedarf für eine derartige Anpassungsoptimierung ist immens, da gerade im dreidimensionalen Fall und bei einer Vielzahl an Kompartimenten eine sehr große Zahl von unbekannten Parametern vorliegt.

\section{Umstellung des Gleichungssystems}

Die Vorgehensweise bei der Umstellung eines Gleichungssystems soll anhand des bereits betrachteten Systems "Nutztierstall” von JANSSEN UND KRAUSE [32] beschrieben werden.

Mit Bezug auf Gleichung 82 stellt sich das ursprüngliche Gleichungssystem in Matrizenform für die Abbildung 19 wie folgt dar:

$$
\left[\begin{array}{cccc}
a_{11} & 0 & a_{31} & a_{41} \\
a_{12} & a_{22} & a_{32} & a_{42} \\
a_{13} & 0 & a_{33} & a_{43} \\
a_{14} & a_{24} & a_{34} & a_{44}
\end{array}\right] \cdot\left[\begin{array}{c}
C_{1} \\
C_{2} \\
C_{3} \\
C_{4}
\end{array}\right]=\left[\begin{array}{l}
V_{1} \cdot \frac{d C_{1}}{d t} \\
V_{2} \cdot \frac{d C_{2}}{d t} \\
V_{3} \cdot \frac{d C_{3}}{d t} \\
V_{4} \cdot \frac{d C_{4}}{d t}
\end{array}\right]
$$

Bei einer Umstellung des ursprünglichen Gleichungssystems (Gl. 82) unter Berücksichtigung der o.g. Ausführungen zur Inversität lautet die erforderliche Schreibweise

$$
X \cdot m=b
$$


$X$ steht nun für die Ausgangsmatrix, welche die Konzentrationswerte enthält; $m$ ist der gesuchte Lösungsvektor; $b$ weiterhin der Ergebnisvektor, welcher die Änderung der Konzentration mit der Zeit bezogen auf das jeweilige Kompartiment beinhaltet. Die bisherigen Hauptdiagonalelemente $a_{i i}$ verschwinden; es verbleiben zehn Austauschkoeffizienten, die es zu bestimmen gilt und zwar

$a_{12}, a_{13}, a_{14}, a_{24}, a_{31}, a_{32}, a_{34}, a_{41}, a_{42}, a_{43}$.

Für die Berechnung dieser zehn unbekannten Austauschkoeffizienten innerhalb eines Zeitschrittes stehen insgesamt vier Bestimmungsgleichungen zur Verfügung. Das bedeutet, die Anzahl an Gleichungen, welche identisch zur Anzahl an Kompartimenten ist, ist geringer als die Anzahl der unbekannten Austauschkoeffizienten. Das Gleichungssystem ist damit unterbestimmt und nicht (eindeutig) lösbar. Um zu einer eindeutigen Lösung zu gelangen, wird angenommen, dass sich die äußeren Einflussgrößen wie z.B. Windrichtung, Windgeschwindigkeit, Temperatur und Druck über mehrere Zeitschritte hinweg nicht bzw. nur geringfügig ändern. Damit können mehrere Konzentrationsänderungen - und damit Bilanzgleichungen - für alle Kompartimente zur Berechnung herangezogen werden. Es werden zunächst soviele Zeitschritte herangezogen, wie Gleichungen zur Lösung des Gesamtsystems benötigt werden. Für das betrachtete Beispiel müssen bei 4 Kompartimenten und 10 Austauschkoeffizienten mindestens 3 Zeitschritte zusammengefasst werden. Die Ausgangsmatrix $X$ besitzt nunmehr folgenden Aufbau:

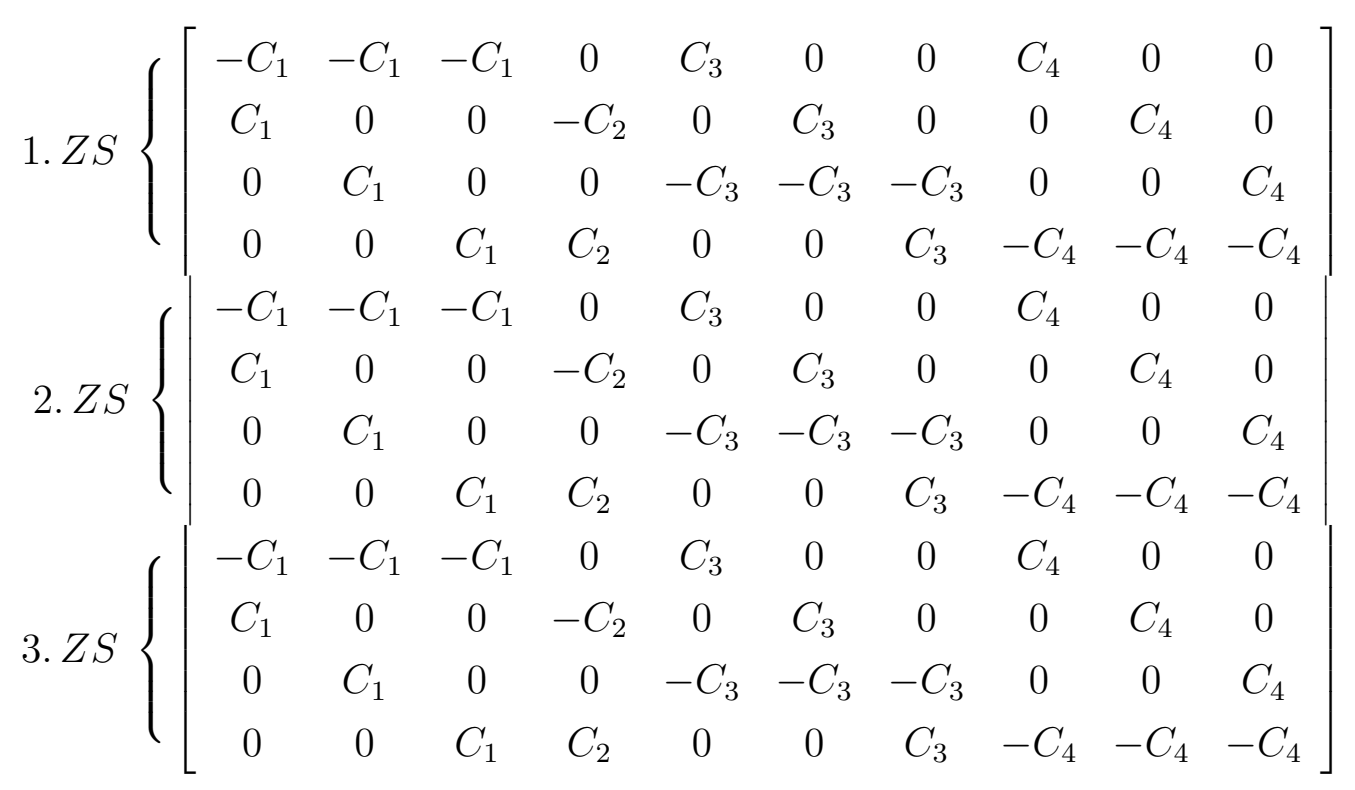

Diese drei "kleinen" Zeitschritte werden als ein "großer" Zeitschritt betrachtet. Das bedeutet, dass die Konstanz der äußeren Einflussgrößen für einen Zeitraum von drei Sekunden (je nach Zeitintervall der Messwerterfassung) vorausgesetzt wird.

Als Zeitschritt wird in diesem Zusammenhang die Zeitspanne zwischen der Erfassung zweier Messwerte bezeichnet. Für jeden hinzukommenden Zeitschritt werden neue Bilanzgleichungen in Höhe 
der Anzahl der Kompartimente gewonnen. Diese Maßnahme ist durch einem Verlust an Information über die Austauschvorgänge im System verbunden, stellt aber eine essentielle Grundlage für die durchzuführenden Berechnungen dar.

In Matrizenform ergibt sich dann das gesamte, umgestellte und überbestimmte Gleichungssystem zu

$$
\left[\begin{array}{cccccccccc}
-C_{1} & -C_{1} & -C_{1} & 0 & C_{3} & 0 & 0 & C_{4} & 0 & 0 \\
C_{1} & 0 & 0 & -C_{2} & 0 & C_{3} & 0 & 0 & C_{4} & 0 \\
0 & C_{1} & 0 & 0 & -C_{3} & -C_{3} & -C_{3} & 0 & 0 & C_{4} \\
0 & 0 & C_{1} & C_{2} & 0 & 0 & C_{3} & -C_{4} & -C_{4} & -C_{4} \\
-C_{1} & -C_{1} & -C_{1} & 0 & C_{3} & 0 & 0 & C_{4} & 0 & 0 \\
C_{1} & 0 & 0 & -C_{2} & 0 & C_{3} & 0 & 0 & C_{4} & 0 \\
0 & C_{1} & 0 & 0 & -C_{3} & -C_{3} & -C_{3} & 0 & 0 & C_{4} \\
0 & 0 & C_{1} & C_{2} & 0 & 0 & C_{3} & -C_{4} & -C_{4} & -C_{4} \\
-C_{1} & -C_{1} & -C_{1} & 0 & C_{3} & 0 & 0 & C_{4} & 0 & 0 \\
C_{1} & 0 & 0 & -C_{2} & 0 & C_{3} & 0 & 0 & C_{4} & 0 \\
0 & C_{1} & 0 & 0 & -C_{3} & -C_{3} & -C_{3} & 0 & 0 & C_{4} \\
0 & 0 & C_{1} & C_{2} & 0 & 0 & C_{3} & -C_{4} & -C_{4} & -C_{4}
\end{array}\right] \cdot\left[\begin{array}{c}
a_{12} \\
a_{13} \\
a_{14} \\
a_{24} \\
a_{31} \\
a_{32} \\
a_{34} \\
a_{41} \\
a_{42} \\
a_{43}
\end{array}\right]=\left[\begin{array}{c}
V_{1} \cdot \frac{d C_{1}(1)}{d t} \\
V_{2} \cdot \frac{d C_{2}(1)}{d t} \\
V_{3} \cdot \frac{d C_{3}(1)}{d t} \\
V_{4} \cdot \frac{d C_{4}(1)}{d t} \\
V_{1} \cdot \frac{d C_{1}(2)}{d t} \\
V_{2} \cdot \frac{d C_{2}(2)}{d t} \\
V_{3} \cdot \frac{d C_{3}(2)}{d t} \\
V_{4} \cdot \frac{d C_{4}(2)}{d t} \\
V_{1} \cdot \frac{d C_{1}(3)}{d t} \\
V_{2} \cdot \frac{d C_{2}(3)}{d t} \\
V_{3} \cdot \frac{d C_{3}(3)}{d t} \\
V_{4} \cdot \frac{d C_{4}(3)}{d t}
\end{array}\right]
$$

Um ein bestimmtes Gleichungssystem zu erhalten, müssen z.B. die Bilanzgleichungen des 3. "kleinen" Zeitschrittes für die Kompartimente 3 und 4 entfernt werden. Dieser Weg wird im Rahmen der Arbeit zuerst beschritten.

Unter der Prämisse, dass sich die bereits genannten äußeren Einflussgrößen über einen längeren Zeitraum nicht oder nur geringfügig ändern, können über die notwendigen Zeitschritte (3 im Beispielfall) hinaus weitere Zeitschritte für die Berechnung der Austauschkoeffizienten herangezogen werden. Es existieren dann sehr viel mehr Gleichungen als Unbekannte; das Gleichungssystem ist wiederum überbestimmt und kann als solches gelöst werden. Die Genauigkeit der Lösung steigt, wie auch die Rechenzeit, mit zunehmender Anzahl an Bestimmungsgleichungen.

Zur Lösung von linearen Gleichungssystemen existieren zahlreiche Rechenverfahren, auf die in dieser Arbeit nicht eingegangen wird. Verwendet werden zunächst der Gauß-Algorithmus bzw. GaußJordan-Algorithmus zur Berechnung bestimmter Gleichungssysteme. Schlägt die Berechnung mit diesen Algorithmen fehl, wird die Householder-Transformation verwendet, womit überbestimmte Gleichungssysteme gelöst werden können. Näheres zur Struktur und zur Vorgehensweise bei der Verwendung der genannten Algorithmen findet sich in [19], [28] bzw. [14]. 


\subsection{Unterschied zur Abklingmethode}

Die Kompartimentalisierungsmethode unterscheidet sich von praxisüblichen Methoden zur Berechnung von Luftvolumenströmen mit Tracern insbesondere in den Ausgangsbedingungen. Hier basiert die Methode auf prinzipiell verschiedenen Annahmen.

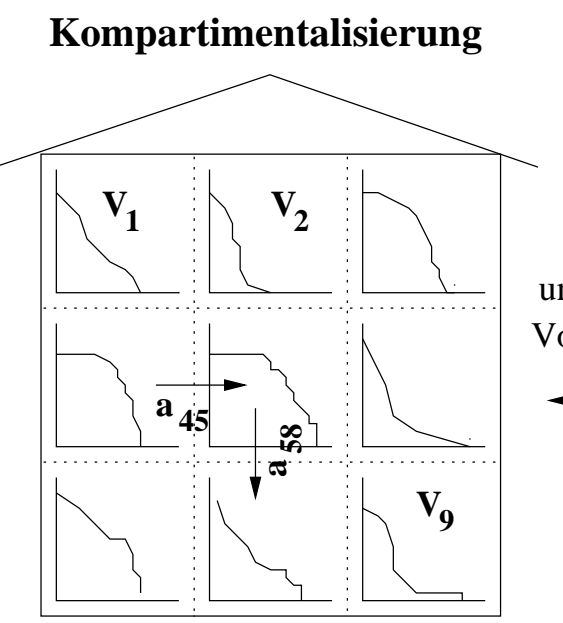

Annahme:

inhomogene Tracerverteilung im System homogene Tracerverteilung im Kompartiment zeitliche Abhängigkeit der Tracerkonzentration
Abklingmethode

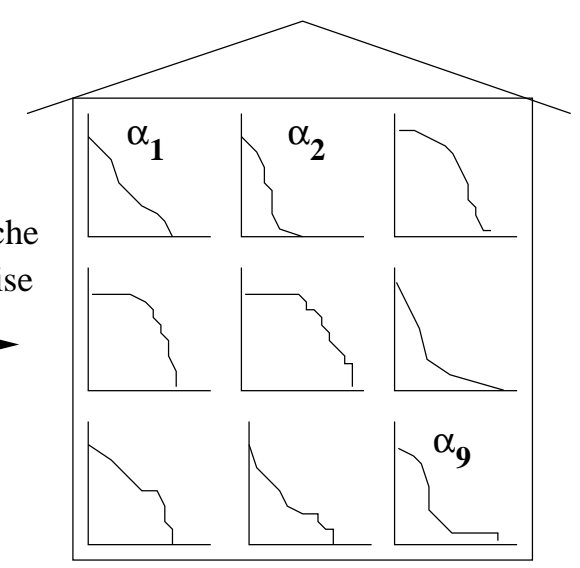

Annahme:

homogene Tracerverteilung im System zeitliche und örtliche

Abhängigkeit der Tracerkonzentration

$$
\begin{gathered}
\bar{\alpha}=\frac{\alpha_{1}+\ldots+\alpha_{n}}{n} \\
\dot{V}_{\text {System }}=\bar{\alpha} \cdot V_{\text {System }}
\end{gathered}
$$

Abbildung 25: Prinzipieller Unterschied zwischen der Kompartimentalisierungsmethode und der Abklingmethode.

Bei der Kompartimentalisierungsmethode (Abb. 25) wird eine inhomogene Verteilung des Tracers innerhalb des Systems angenommen. Diese Annahme gestaltet sich im Hinblick auf die unternommenen Vorüberlegungen in Kapitel 2.1.4 realitätsnah. Das System wird in virtuelle Kompartimente unterteilt, wobei die Volumengrößen variieren können. Im Zentrum jedes Kompartimentes befindet sich ein Sensor, der das Abklingen der Tracerkonzentration mit der Zeit aufzeichnet. Innerhalb eines Kompartimentes wird die Tracerverteilung als homogen angenommen, d.h. die örtliche Abhängigkeit der Tracerkonzentration entfällt. Die Tracerkonzentration ändert sich somit "sprunghaft" an den virtuellen Kompartimentsgrenzen. Um die Bilanzgleichungen des Tracers für jedes Kompartiment und die Kontinuitätsgleichung für das System zu erstellen, ist es erforderlich, (Konzentrations-)Gradienten zu bilden, welche anhand der Abklingreihen des Tracers zur Verfügung stehen. Der Austausch des Tracers über die Kontaktflächen hinaus wird durch die Austauschkoeffizienten beschrieben. Die entsprechende Gleichung ist in der Abbildung 25 dargestellt. Errechnet werden die Volumenströme in Form der genannten Austauschkoeffizienten sowohl zwischen Kompartimenten als auch an der Grenze des 
Systems. Die Bilanzierung der Volumenströme der an der Systemgrenze befindlichen Kompartimente ergibt den Gesamtvolumenstrom des Systems. Durch die internen Austauschkoeffizienten kann der systeminterne Strömungsverlauf charakterisiert werden. Das Kompartimentalisierungsverfahren wird als unabhängig von wechselnden Windanströmverhältnissen angesehen. Es werden in Abhängigkeit von der Versuchsdauer endlich viele Volumenströme errechnet, die sich jeweils auf einen Zeitschritt des Versuchszeitraumes beziehen. Somit ist es möglich, den variierenden Verlauf des Volumenstromes zu ermitteln.

Die in Abbildung 25 (rechte Hälfte) dargestellte Abklingmethode setzt eine homogene Tracerverteilung im System - nach erfolgter stoßartiger Tracerzugabe - voraus. Nur unter dieser Annahme kann die Luftwechselzahl nach Gleichung 15 berechnet werden! Da der idealisierte Ansatz einer homogenen Durchmischung eines Systems in der Realität ungeeignet ist, ist die Volumenstromberechnung mit einem Fehler behaftet. Inwieweit es zur Fehlerfortpflanzung kommt, hängt von den vorherrschenden meteorologischen Gegebenheiten und der Positionierung der Messpunkte ab.

Jeder Sensor liefert eine Messreihe, wonach über eine geschätzte Abklingfunktion die mittlere Luftwechselzahl an diesem Messpunkt ermittelt wird (Abb. 25 rechts unten). Diese mittlere Luftwechselzahl wird als allgemeingültig für den gesamtem Versuchszeitraum angenommen, da die Abklingfunktionen der einzelnen Messpunkte in der Regel unterschiedliche Steigungen zu unterschiedlichen Zeitpunkten aufweisen. Differenzierte Aussagen zum Volumenstrom des Systems in einzelnen Zeitabschnitten sind aufgrund dieser zeitlichen Verschiebung sinnlos. Ebensowenig können Aussagen zum Strömungsverlauf gemacht werden, man erhält nur einen Volumenstrom für das System ("Glockenprinzip").

Die Kompartimentalisierungsmethode weist somit im Vergleich zu üblichen Tracermethoden grundsätzliche Unterschiede auf. Zunächst wird in Anlehnung an die Abklingmethode ebenfalls ein Tracer stoßartig freigesetzt und das Abklingverhalten mit der Zeit aufgezeichnet. Die Auswertung und Interpretation der Zeitreihen erfolgen jedoch in Anlehnung an die strömungsmechanischen Gesetzmäßigkeiten. Ausgehend von diesen elementaren physikalischen Kriterien wird der Volumenstrom für jeden einzelnen Zeitschritt in Form eines gekoppelten Differentialgleichungssystems (Gl. 81) errechnet. 


\section{Material}

Um die Praxistauglichkeit der Kompartimentalisierungsmethode zu untersuchen, werden Versuche an zwei ausgewählten Rinderhaltungsanlagen durchgeführt: ein Offenstall mit Schwerkraftlüftung sowie ein Offenfrontstall.

\subsection{Ställe im Feldversuch}

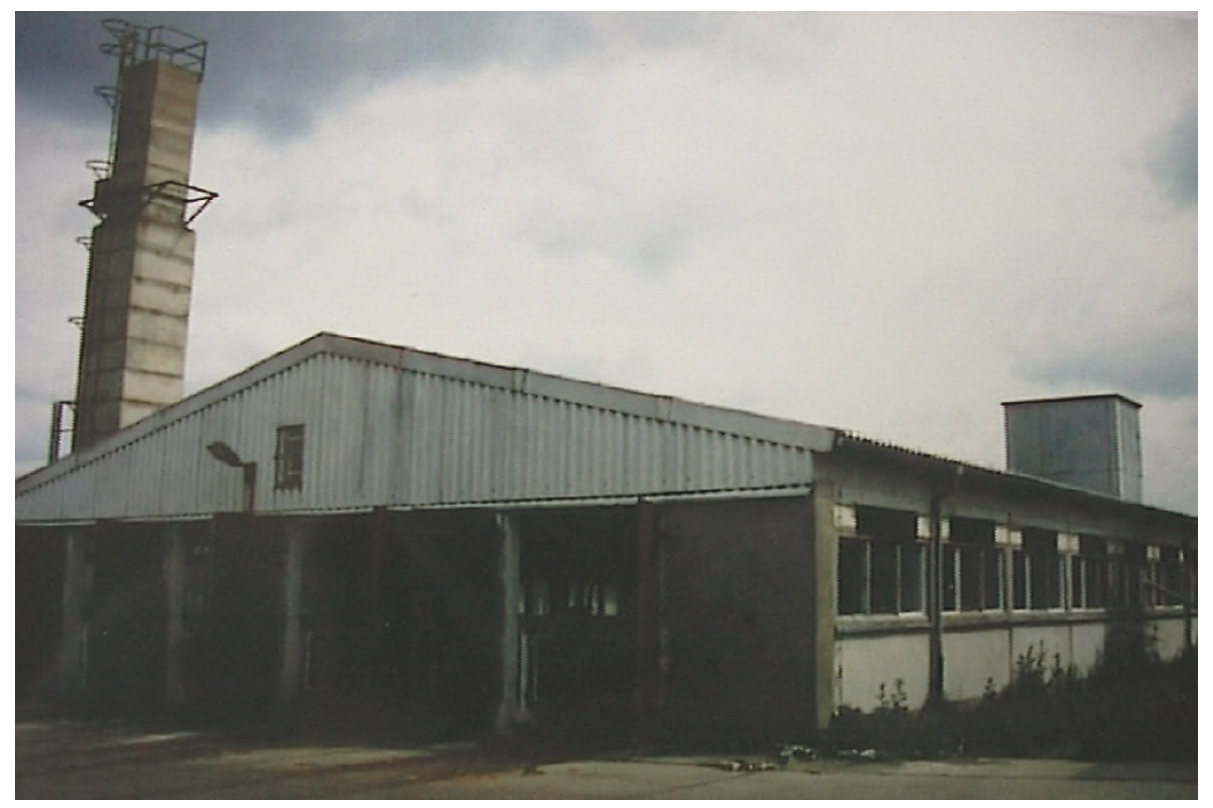

Abbildung 26: Südwestansicht eines Offenstalles mit Schwerkraftlüftung mit einem dachmittig platzierten Abluftschacht in Faulenhorst (Sachsen-Anhalt). Jeweils vier Tore auf beiden Giebelseiten sind geöffnet, die Fenster an den Seitenwänden sind geschlossen. Der Stallinnenraum besitzt eine geschlossene Zwischendecke in Traufhöhe.

\section{Beispiel 1:}

Abbildung 26 zeigt die Südwestansicht eines Milchviehstalles mit einem Besatz von 135 Kühen und Rindern ( $\emptyset 600$ kg LG $\Rightarrow 162$ GV). Der Stall ist ein Teil der Tierproduktionsanlage der Milchgenossenschaft Faulenhorst im Kreis Salzwedel (Sachsen-Anhalt), einer ehemaligen LPG. Das Gebäude ist 63,00 $\mathrm{m}$ lang und 15,72 $\mathrm{m}$ breit. Im Innern ist eine Zwischendecke in 3,15 $\mathrm{m}$ Höhe eingezogen. Dachmittig ist ein 3,35 m x 2,20 m breiter Abluftschacht positioniert. Im Stall, in Höhe des Abluftschachtes, bestehen Durchgänge zum Melkstand bzw. zu einem benachbarten Stall. Die Stalllängsseiten verlaufen in Ost-West-Richtung und weisen eine durchgängige, geschlossene Fensterfront auf. An den Giebelseiten sind jeweils vier Tore von 2,40 m Breite und 3,00 m Höhe vorhanden. 

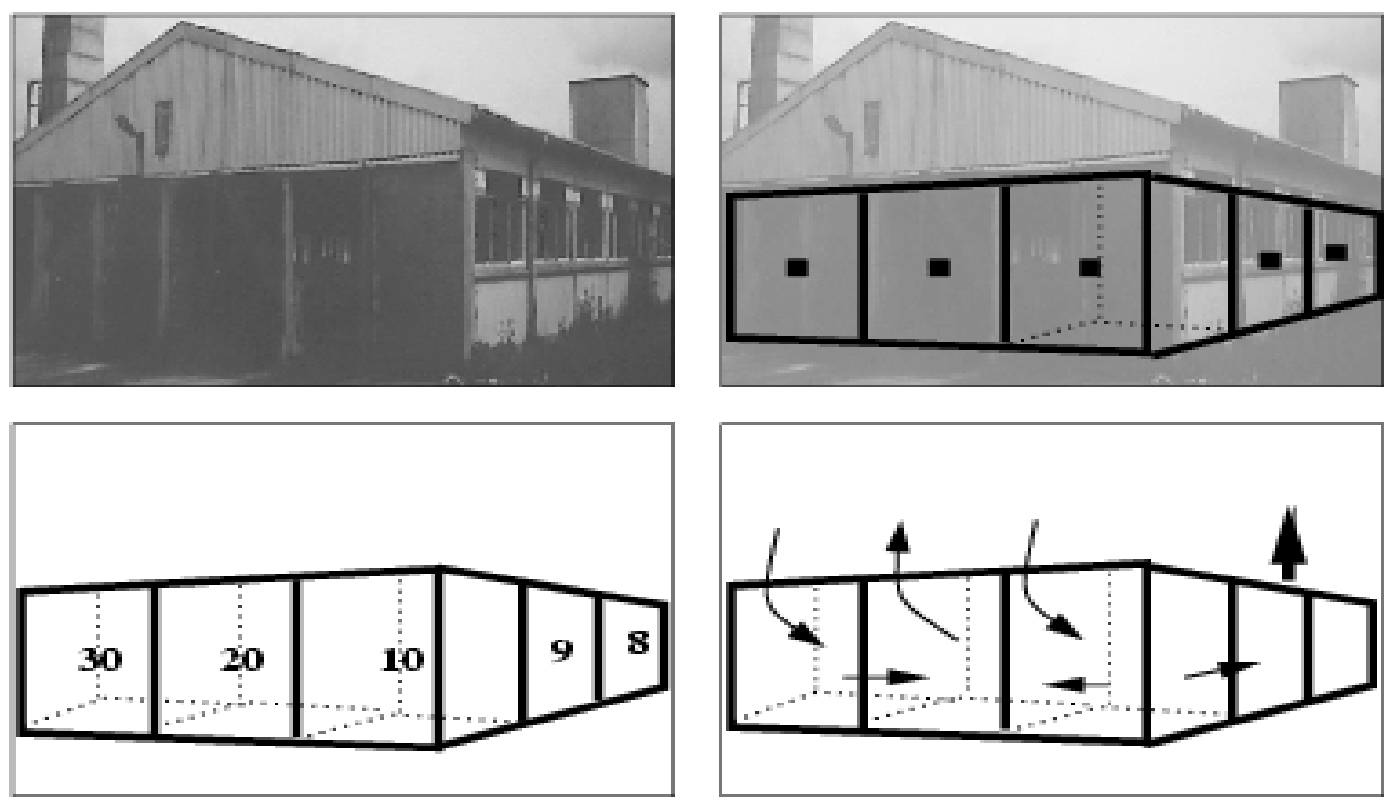

Abbildung 27: Prinzipielle Vorgehensweise bei der Kompartimentalisierung von Ställen am Beispiel des Stalles in Faulenhorst. Anhand der Positionen der Sensoren im kartesischen Koordinatensystem des Stalles wird der Stall in Kompartimente unterteilt. Die Verbindungen zwischen den Kompartimenten (siehe auch Kapitel 5.4) werden festgelegt und die Austauschkoeffizienten berechnet. Die errechneten Werte für diesen Fall sind in Abbildung 39, Kapitel 6 zu sehen.

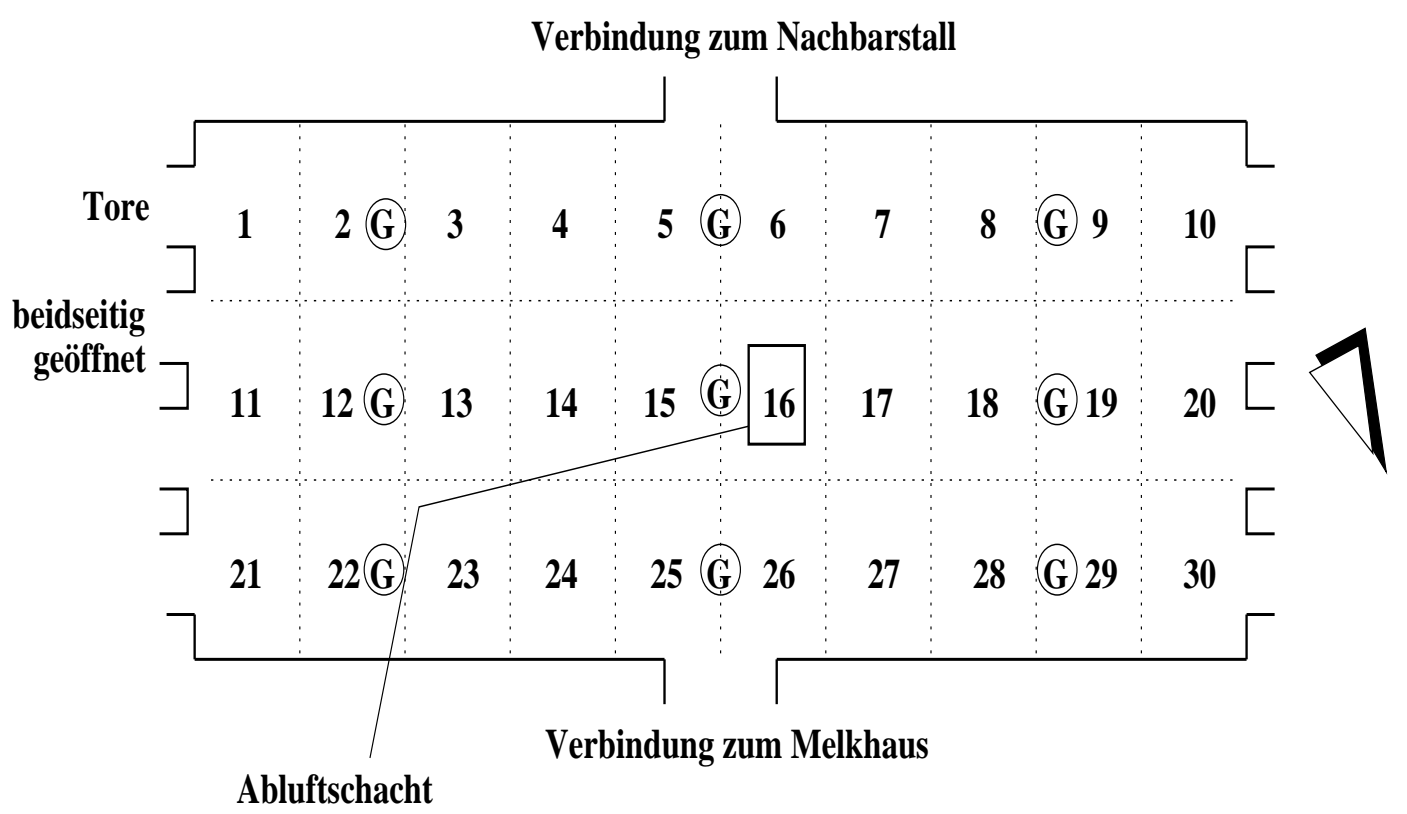

Abbildung 28: Lage und Anordnung der Kompartimente (1 bis 30) im untersuchten Offenstall mit einem dachmittig platzierten Abluftschacht (Faulenhorst, Sachsen-Anhalt). Potenzielle Ein- bzw. Austrittsorte der Luft sind eingezeichnet. Horizontaler Querschnitt. Mit "G" sind die Orte der Gaskonzentrationserfassung gekennzeichnet. 
Der Stall ist ein Boxenlaufstall mit befestigter Trittfläche. Stroh dient als Boxeneinstreumaterial, entmistet wird mittels Radlader auf eine östlich gelegene Dungplatte.

Für die Volumenstrombestimmung wird der Stall in 30 Kompartimente unterteilt, d.h. es werden 30 Tracer-Sensoren gleichmäßig im Stall (Höhe über Erdboden: 2,50 m) verteilt angebracht. Anhand der Abbildung 27 soll dabei noch einmal die Vorgehensweise bei der Kompartimentalisierung verdeutlicht werden. Das Stallvolumen beträgt $3.045 \mathrm{~m}^{3}$. Damit besitzt jedes Kompartiment ein durchschnittliches Volumen von rund $100 \mathrm{~m}^{3}$. Die Aufzeichnung der Tracerkonzentration erfolgt stets im Zentrum eines jeden Kompartimentes. Die genaue Anordnung und Lage der Kompartimente im System zeigt Abbildung 28. Potentielle Ein- bzw. Austrittsorte der Luft sind eingezeichnet.

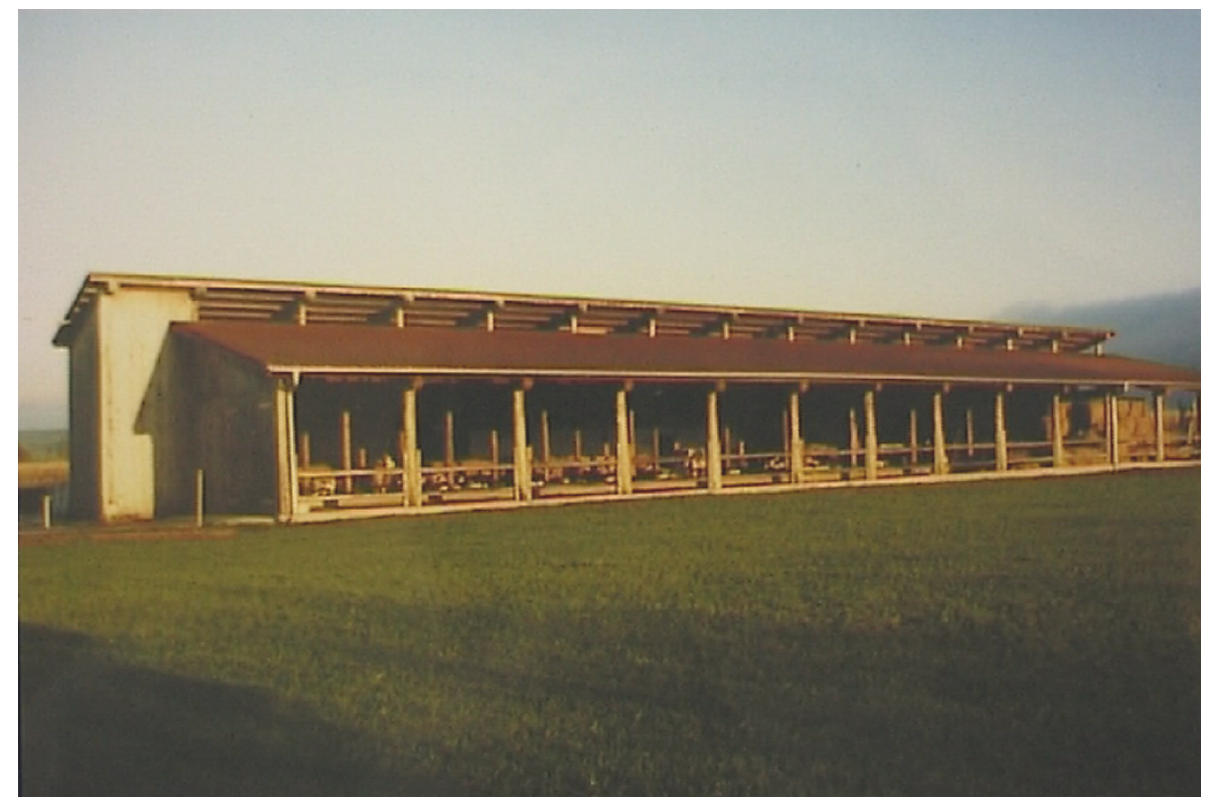

Abbildung 29: Ansicht der südlichen Längsseite eines Offenfrontstalles in Rothenstadt (Bayern). Die der offenen Seite gegenüber befindliche Rückwand weist einen durchgehenden Öffnungsschlitz von $0,50 \mathrm{~m}$ in Traufhöhe auf.

Beispiel 2:

Abbildung 29 zeigt einen Offenfrontstall mit Spaceboard an den Giebelseiten (siehe hierzu Abbildung 1 in der Einleitung). Der Stall befindet sich in Rothenstadt, Kreis Weiden (Bayern). 39 Kühe $(\emptyset 600$ kg LG) entsprechen einem Tierbesatz von 46,8 GV. Das Stallgebäude ist 36,20 m lang, 22,30 m breit und am obersten Punkt des Pultdaches 6,17 m hoch. Der Stall ist als nicht wärmegedämmter Stall ausgelegt, die Entmistung des Boxenlaufstalles erfolgt über Spaltenböden. Die nordwestliche Längsseite weist eine durchgängige Schlitzöffnung von ca. 0,50 m in Traufhöhe auf; an dieser Stallseite befinden sich auch der Melkstand und ein Büroraum.

Das Stallvolumen beträgt $3.411 \mathrm{~m}^{3}$, die 15 eingesetzten Sensoren zur Bestimmung des Luftvolumenstromes sind ebenfalls gleichmäßig und in einer einheitlichen Höhe von 2 m über dem Erdboden 


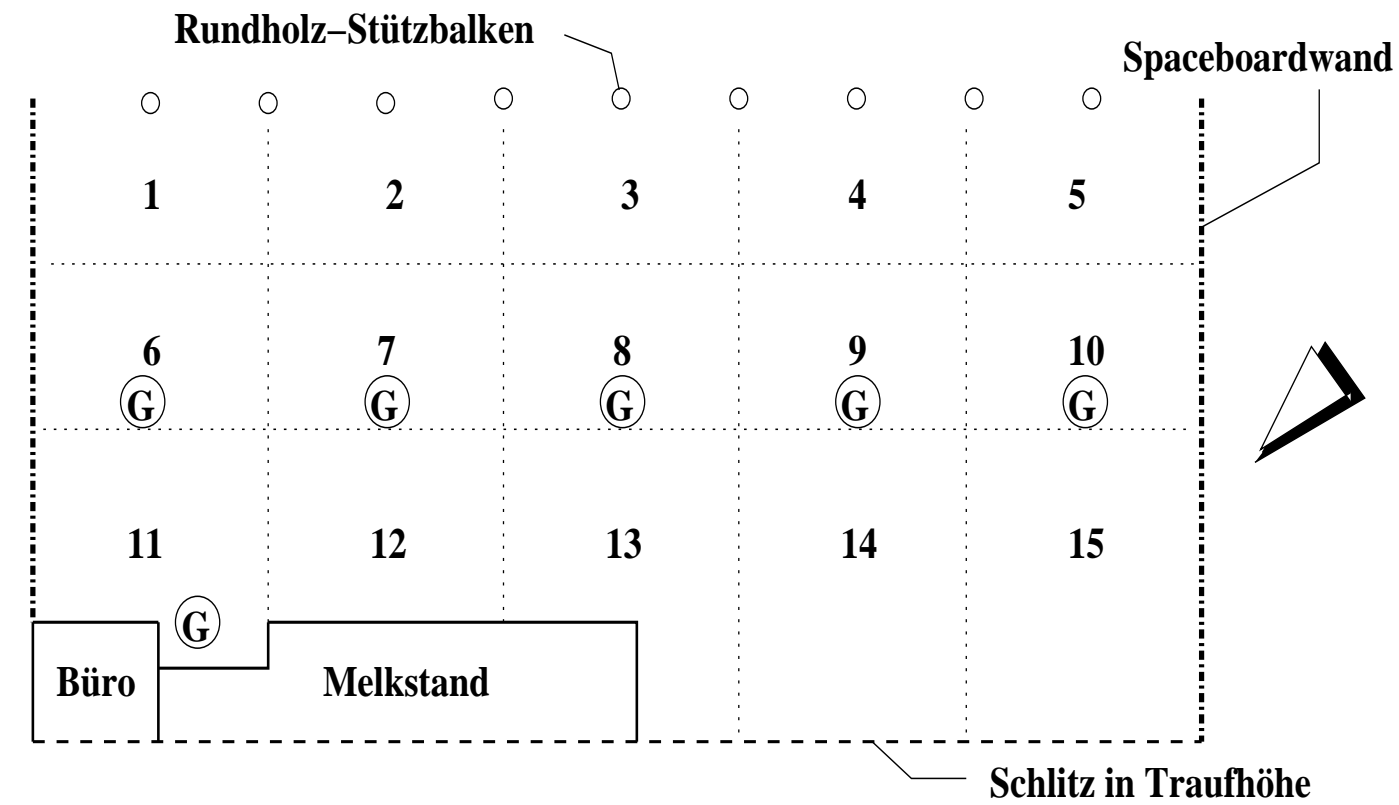

Abbildung 30: Lage und Anordnung der Kompartimente (1 bis 15) im untersuchten Offenfrontstall mit giebelseitigem Spaceboard (Rothenstadt, Bayern). Potenzielle Ein- bzw. Austrittsorte der Luft sind eingezeichnet. Horizontaler Querschnitt. Mit "G" sind die Orte der Gaskonzentrationserfassung gekennzeichnet.

angebracht. Das durchschnittliche Kompartimentvolumen beträgt rund $230 \mathrm{~m}^{3}$; es treten aber erhebliche Abweichungen zwischen den einzelnen Kompartimentvolumina auf. Die unterschiedliche Größe der Kompartimente wird bei der Berechnung des Luftvolumenstromes berücksichtigt.

Die Anzahl der verwendeten Sensoren hängt in beiden Fällen nicht nur von den baulichen Gegebenheiten (i.e. Größe des Stalles) ab, sondern beruht vor allem auf der Tatsache, dass maximal 80 Sensoren zur Verfügung stehen. Des weiteren ist es notwendig, einen ausreichenden Zwischenraum zwischen zwei Sensoren bzw. zwischen Sensor und Wand zu gewährleisten. Ursache ist das vorhandene "Einzugsgebiet" eines jeden Sensors von ca. $1 \mathrm{~m}$, innerhalb dessen sich aus Genauigkeitsgründen kein weiterer Sensor befinden sollte. Anordnung und Lage der Kompartimente verdeutlicht Abbildung 30.

\subsection{Ablauf der Tracergasmessung}

Als Tracer wird das radioaktive Edelgas Krypton 85 eingesetzt (siehe hierzu [47]). Gegenüber konventionellen Tracern wie z.B. $\mathrm{CO}_{2}$ oder $\mathrm{H}_{2} \mathrm{O}$ hat Krypton den Vorteil, dass es keinen tageszeitlichen Schwankungen (etwa durch Tieraktivität) unterliegt bzw. mit der verfügbaren Messtechnik (im Vergleich zu SF 6 ) zeitlich hoch auflösend erfasst werden kann. Die Abtastfrequenz beträgt $1 \mathrm{~Hz}$ parallel an allen Messpunkten. Das ist im Hinblick auf die gemachten Vorüberlegungen in Kapitel 2.1.4 zur zeitgleichen Messwerteerfassung ein entscheidender Vorteil. Die notwendige Strahlungsaktivität 
(StA) zum Beginn einer Tracermessung ist abhängig von der Größe des Stalles sowie von der Anzahl und Größe der Öffnungen. Für die unter 5.1 beschriebenen Realställe ist jeweils eine Startkonzentration (= Zählimpulse) an Krypton von ca. $10.000 \frac{\mathrm{Impulse}}{\mathrm{min} \cdot \mathrm{m}^{3}}$ erforderlich. Dies entspricht einer StA von etwa $2 G B q$ für den gesamten Stallraum. Das heißt, die volumenbezogene Anfangsaktivität beträgt im Fall Faulenhorst $\frac{2000 M B q}{3045 m^{3}}=0,67 \frac{M B q}{m^{3}}$ und im Fall Rothenstadt $\frac{2000 M B q}{3411 m^{3}}=0,59 \frac{M B q}{m^{3}}$.

Erfasst wird die Kryptonkonzentration in Form von Zählimpulsen mittels Beta-Gamma-Zählrohren (Fa. Vacu Tech), welche eine Fehlertoleranz von ca. $3 \frac{\text { Impulse }}{\mathrm{min} \cdot \mathrm{m}^{3}}$ aufweisen. Die Zählimpulse werden prozessgesteuert registriert und per Funk an eine Master-Station übertragen [48], die mit einem Rechner gekoppelt ist. Jeweils vier Zählrohre sind an einem von zehn zur Verfügung stehenden Funkdatenloggern (Slaves) angeschlossen. Infolge der hohen zeitlichen Messwerteerfassung kann die Änderung der Tracerkonzentration quasi kontinuierlich bestimmt werden.

Vor Beginn jeder Volumenstrombestimmung werden schließbare Öffnungen verschlossen, um den Tracer nicht bereits während der Ausbringphase entweichen zu lassen. Die Dosierung des Tracers erfolgt mittels zweier Glasbehälter, welche in vertikaler Richtung durch ein Ventil miteinander verbunden sind. Im unteren Glasbehälter befindet sich das Krypton-Tracergas in der erforderlichen Strahlungsaktivität, der obere Behälter ist mit einer definierten Menge an Wasser gefüllt. Sodann wird das Wasser kontinuierlich über das Ventil in den unteren Behälter gelassen, bis es sich vollständig in diesem befindet. Dadurch kommt es zur Verdrängung des Tracers, der über eine weitere Öffnung direkt in den Stallraum injiziert wird. Um eine möglichst homogene Tracerverteilung zu erzielen, wird der Stall mit den beiden Glasbehältern gleichmäßig durchlaufen, wobei die Verteilung des Tracers im Stallraum zusätzlich durch Wedelbewegungen mit Holzschildern unterstützt wird.

In den vorgestellten Rinderanlagen werden zwei (Faulenhorst) bzw. vier Tracerversuche (Rothenstadt) durchgeführt. Der Ergebnisteil (6) beschränkt sich jeweils auf den ersten Versuch. Parallel zur Volumenstrombestimmung werden zum einen die Gaskonzentrationen an $\mathrm{NH}_{3}, \mathrm{CH}_{4}, \mathrm{CO}_{2}, \mathrm{~N}_{2} \mathrm{O}$ und $\mathrm{H}_{2} \mathrm{O}$ in $2 \mathrm{~m}$ Höhe an den in Abbildung 28 bzw. 30 eingezeichneten Orten gemessen; zum anderen erfolgen Begehungen im Umfeld der Ställe, um auf die Geruchsbelastung bzw. -belästigung zu schließen. In unmittelbarer Nähe der Ställe ist ein $10 \mathrm{~m}$ hoher Windmast aufgebaut, welcher die dortige Windgeschwindigkeit und Windrichtung aufzeichnet. Zur Interpretation der errechneten Volumenströme werden die Windgeschwindigkeiten später auf 2 m Höhe umgerechnet. Im Stall und außerhalb des Stalles sind Temperatur- und Feuchtemessgeräte in ca. 2 m Höhe angebracht.

\subsection{Konzentrationsbestimmung}

Die zweite erforderliche Komponente zur Quantifizierung von gasförmigen Emissionen ist neben dem Volumenstrom die Gaskonzentration. Konzentrationsmessungen müssen, wie bereits in Kapitel 2.2 erwähnt, parallel zur Volumenstrombestimmung erfolgen. 
In der landwirtschaftlichen Nutztierhaltung entstehen Gase primär durch den intermediären Stoffwechsel (z.B. $\mathrm{CO}_{2}, \mathrm{CH}_{4}$ ) und/oder aus den Ausscheidungen und sekundär nachgeschalteten Reaktionen in den Exkrementen (z.B. $\mathrm{NH}_{3}, \mathrm{~N}_{2} \mathrm{O}$ ). Es ist daher nach einem Messgerät gefragt, welches in der Lage ist, alle relevanten Gaskonzentrationen aus der Rinderhaltung kontinuierlich zu erfassen. Im Rahmen dieser Arbeit wird das photoakustische Messgerät 1302 der Firma Innova Airtech Instruments (ehemals Brüel \& Kjær) verwendet, dessen wesentliches Funktionsprinzip im Folgenden kurz beschrieben wird.

a)

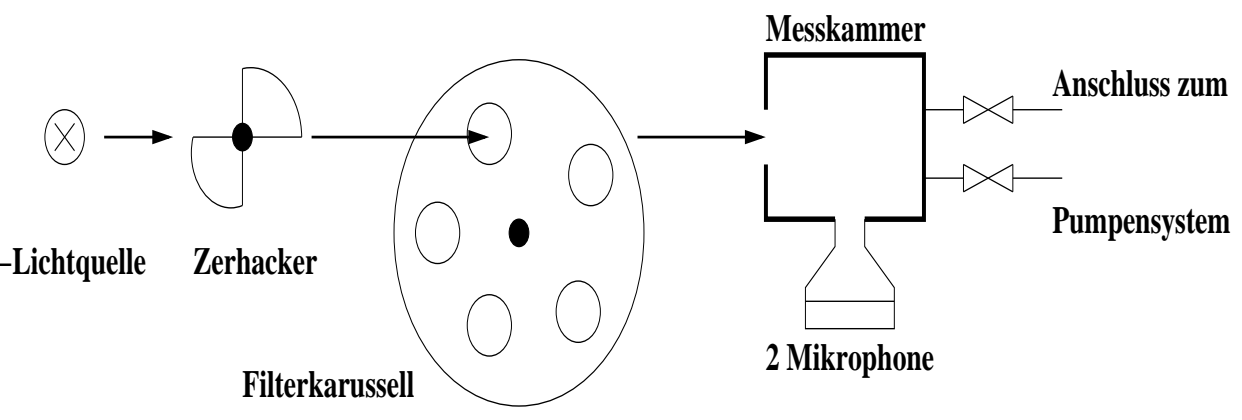

b) Lichtintensität in der Messkammer

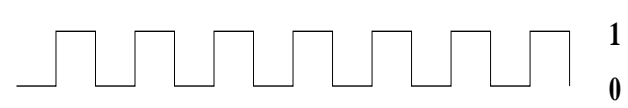

Temperatur- u. Druckverlauf in der Messkammer

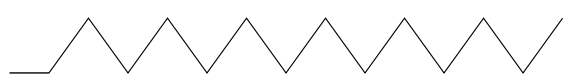

Abbildung 31: Aufbau des photoakustischen Gasanalysators 1302, Fa. INNOVA Air Tech Instruments [64].

Das Messgerät 1302 (siehe Abb. 31) besteht aus einer Infrarot (IR)-Lichtquelle, einem Zerhacker, einem Filterkarussell mit verschiedenen optischen Filtern und einer Messkammer mit zwei Mikrophonen. Des weiteren enthält das Gerät eine elektronische Steuereinheit sowie ein Pumpensystem, das die Gaszelle mit dem zu untersuchenden Gas befüllt.

Photoakustische Spektroskopie nutzt die Tatsache aus, dass ein eingeschlossenes Gasgemisch Schallwellen aussendet, nachdem es zerhacktes Licht einer IR-Quelle absorbiert hat. Der Zerhacker liefert gepulstes Licht, aus welchem mittels geeigneter optischer Filter Spektralbereiche (Wellenzahlen) von $20 \mathrm{~cm}^{-1}$ bis $100 \mathrm{~cm}^{-1}$ ausgewählt werden. Das IR-Licht des gewählten Spektralbereichs wird in die Messkammer geleitet und dort von Gasen absorbiert, die genau in diesem Spektralbereich IRaktiv sind. Das heißt, durch die Bestrahlung des Gases wird die absorbierte Lichtenergie sofort in Form von Wärme frei (periodische Änderung der Temperatur), welches eine Druckerhöhung in der Messkammer nach sich zieht. Die zwei in die Messkammerwand eingebauten Mikrophone detektieren die Druckschwankungen und leiten sie an die elektronische Steuereinheit weiter, die das Signal 
auswertet. Die Amplitude der Druckschwankungen ist proportional zur Konzentration des Gases im Probengemisch und somit ein Maß für den quantitativen Anteil dieses Gases in der Stallluft.

Nach erfolgter Konzentrationsbestimmung eines Gases dreht das Filterkarussell zum nächsten optischen Filter, ein anderes Gas der angesaugten Probe wird untersucht. Durch den Einsatz unterschiedlicher optischer Filter ist es möglich, verschiedene Gase zu bestimmen und bekannte Querempfindlichkeiten zu eliminieren. Querempfindlichkeiten treten mit allen Gasen auf, die im speziellen Spektralbereich des untersuchten Gases ebenfalls IR-aktiv sind. Für Wasserdampf ist im 1302 standardmäßig eine Kompensation eingebaut.

Die Häufigkeit des Rotierens des Filterkarussells hängt von der Anzahl der Gaskomponenten im Gasgemisch ab. Bei der Analyse von nur einer Gaskomponente einer Probe aus unmittelbarer Gerätenähe beträgt die Messzeit des Gerätes ca. 30 Sekunden. Da punktuelle Gasmessungen in der Regel wenig aussagekräftig sind, wird der Monitor 1302 in Verbindung mit dem Mehrpunktprobennehmer und Dosierer 1303 als System eingesetzt. Bei vier zu bestimmenden Gaskonzentrationen sowie der Ermittelung des Wasserdampfgehalts der Probe, wie im Rahmen dieser Arbeit als Ziel formuliert, vergrößert sich die Messzeit des Gerätes auf ca. 120 Sekunden. Der Monitor 1302 eignet sich damit zur Bestimmung der Gaskonzentrationen aus der Rinderhaltung, wenngleich die Messwerterfassung diskontinuierlicher Natur ist. Bei längeren Messzeiträumen sind allerdings eine regelmäßige Kalibrierung des Gerätes und die Kompensation von Querempfindlichkeiten (insbesondere zu Wasserdampf) unverzichtbar, da sonst erhebliche Abweichungen im Messverlauf auftreten bzw. fehlerhafte Gaskonzentrationen gemessen werden [67].

\subsection{Software und Programmablauf}

Zur Berechnung des Volumenstromes in frei gelüfteten Systemen wird eine selbst programmierte Software unter Windows (C) (Visual Basic (C) verwendet. Volumenstromberechnungen in zwangsbelüfteten Ställen sind ebenfalls möglich. Einen Blick auf die Bedienoberfläche des Programms vermittelt Abbildung 32.

Die prinzipielle Vorgehensweise, wie sie in Abbildung 33 skizziert ist, gestaltet sich wie folgt:

1. Anhand der unveränderlichen geometrischen Randbedingungen Länge, Breite und Höhe wird die Größe des Gesamtsystems ermittelt.

2. Die Position eines jeden Sensors ist im kartesischen Koordinatensystem eindeutig festgelegt. Die Koordinaten der Sensoren dienen dem Zweck, das in eine finite Anzahl von Volumenelementen zerlegte Gesamtsystem auf die einzelnen Kompartimente (=Anzahl der Sensoren) 


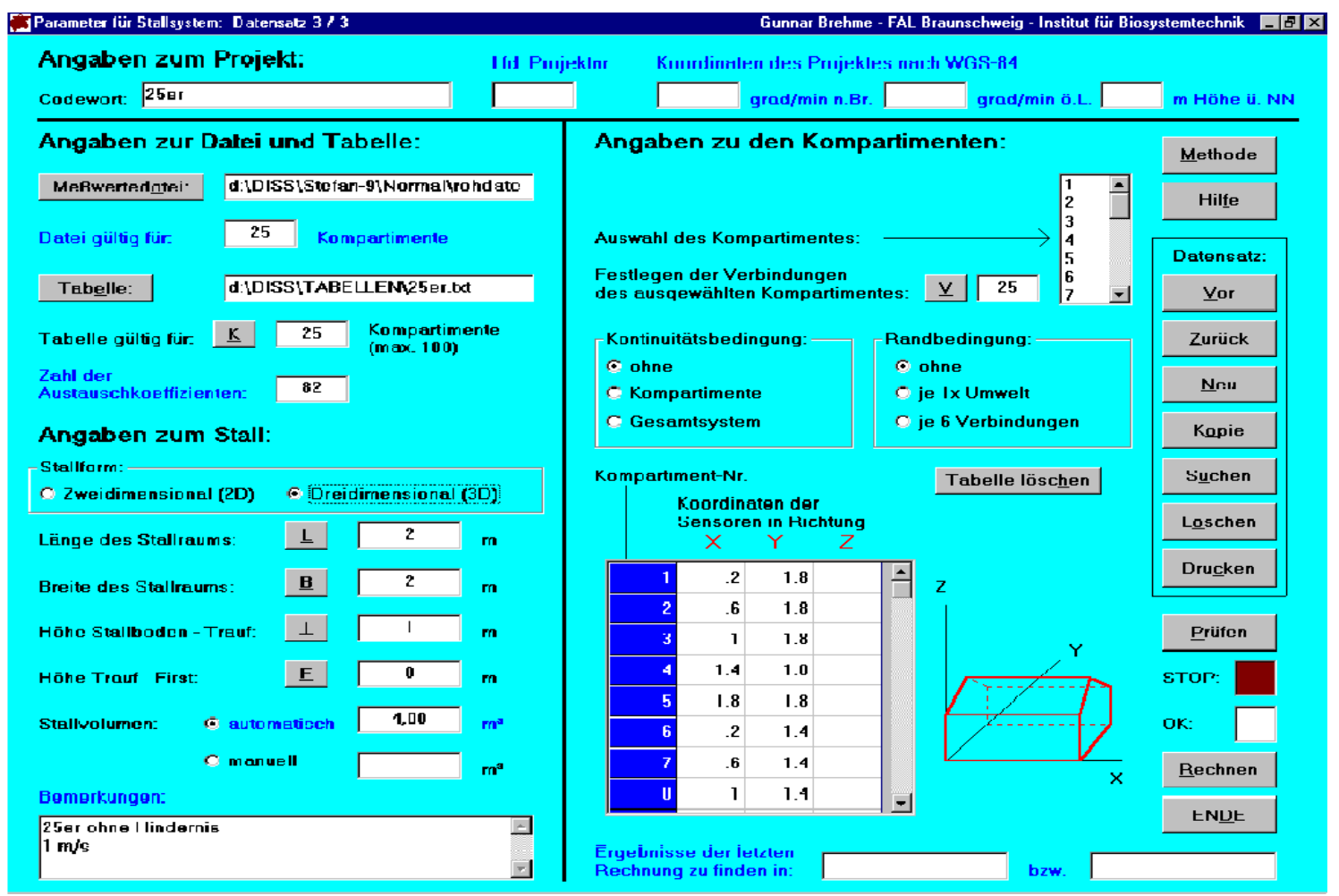

Abbildung 32: Haupteingabemaske der eigenen Software zur Berechnung des Luftvolumenstromes.

aufzuteilen. Hierbei wird die Kompartimentsgröße mittels Methode der kleinsten Entfernungen bestimmt. Durch die ermittelte Position der Kompartimente zueinander sind auch deren Verbindungen festgelegt.

3. Eventuell auftretende stallinterne Hindernisse werden mittels MAC-Ansatz ("marker and cell") bestimmt, d.h. anhand der Koordinaten der Eckpunkte eines Hindernisses (ausschließlich Hexaederform) im zugrunde liegenden kartesischen Koordinatensystem kann ein Hindernis im System eindeutig definiert werden. Öffnungen werden in analoger Weise festgelegt.

4. Die aufgenommenen Zeitreihen der Tracerkonzentration aller Messpunkte werden parallel eingelesen; die zeitliche Änderung der Tracerkonzentration wird berechnet.

5. Nach Eingabe der Kontinuitäts- und/oder Randbedingung wird unter Berücksichtigung von Punkt 2 die Anzahl der sich ergebenden Austauschkoeffizienten errechnet. Die Zahl dieser Unbekannten bleibt im weiteren Rechenverlauf unverändert.

6. Die Länge der eingelesenen Zeitreihen ist endlich. Daraus sowie durch die bekannte Zahl an Austauschkoeffizienten ergibt sich die maximale Anzahl an Zeitschritten. $\Delta T$ ist in diesem Zusammenhang als "großer" Zeitschritt zu betrachten (siehe hierzu auch die Ausführungen in 
Kapitel 4.3.2), in welchem die notwendigen "kleinen" Zeitschritte, d.h. die aufgenommenen Zeitschritte, für die entsprechende Berechnung zusammengefasst sind.

7. Das Gleichungssystem wird zunächst als bestimmtes Gleichungssystem aufgestellt. Ist eine eindeutige Lösung mittels der eingesetzten Algorithmen (Gauß bzw. Gauß-Jordan) nicht möglich, wird auf den Householder-Algorithmus zurück gegriffen; die Reihenfolge ist in Abbildung 33 ersichtlich. Aufgrund der unterschiedlichen Input- und Outputanforderungen, Lösungsprinzipien etc. der verwendeten Algorithmen muss die Anzahl der Zeitschritte neu berechnet werden. Ist keine Lösung möglich, bricht das Programm ab.

8. Existiert eine Lösung für einen Zeitschritt $\Delta T$, wird das Ergebnis abgespeichert. Es werden sowohl der Wert des Volumenstromes für das Gesamtsystem als auch die Höhe der Volumenströme zwischen den Kompartimenten ausgewiesen. Damit sind Aussagen zum Strömungsverlauf möglich.

9. Die Rechnung wird beendet, wenn die maximale Anzahl an Zeitschritten erreicht ist.

Im nachfolgenden Kapitel 6 wird das Kompartimentalisierungsverfahren in mehreren Beispielfällen aus Theorie und Praxis angewendet. Es wird zunächst die Simulationsrechnung der in Kapitel 3.3 vorgestellten Strömungssituation in verschiedenen Variationen nachgefahren und analysiert. Anschließend werden Rechnungen aus den genannten Realställen vorgestellt. 


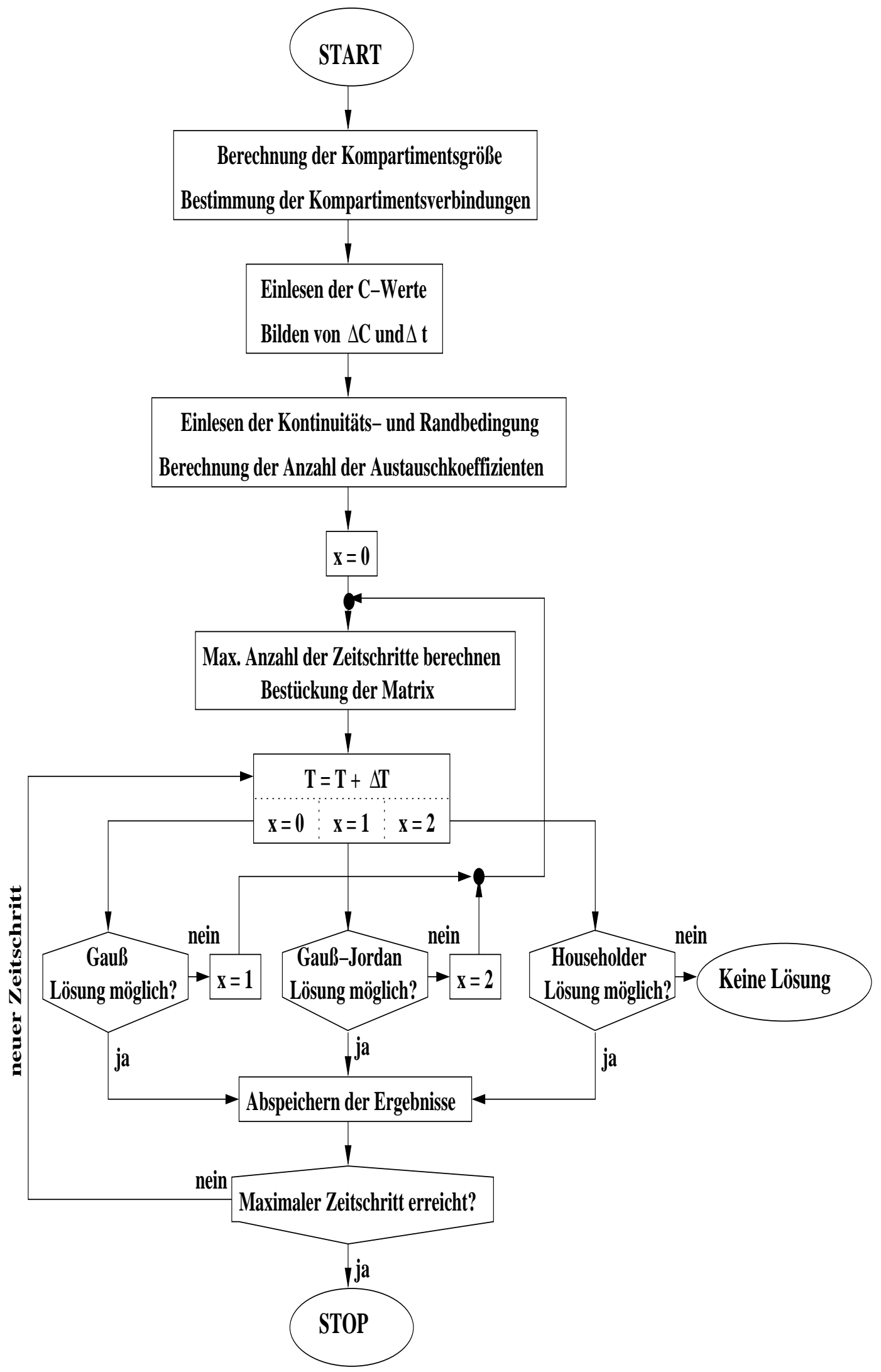

Abbildung 33: Programmablaufplan (PAP) der eigenen Software zur Berechnung des Luftvolumenstromes. 


\section{Ergebnisse}

\subsection{Modellversuche}

Allen Modellversuchen liegt ein zweidimensionaler Raum mit einer Kantenlänge von jeweils $2 \mathrm{~m}$ zugrunde. Nach erfolgter Ausbildung des stationären Strömungsfeldes wird schlagartig ein Tracer $\left(\mathrm{NH}_{3}\right)$ zugegeben. Die Abklingreihen der ausgewählten Messpunkte stellen die einheitliche Berechnungsgrundlage für alle eingesetzten Verfahren dar. Die Aufteilung des Raumes in der Numerik erfolgt in 1.600 Raumpunkte. In diesem Fall werden die Geschwindigkeiten und Richtungen der Punkte verwendet, welche die virtuellen Grenzflächen der Kompartimente beschreiben. Sämtliche Raumpunkte einer virtuellen Austauschfläche - insgesamt jeweils 14 Punkte, aus deren Geschwindigkeitswerten ein Mittelwert errechnet wird - ergeben somit einen Volumenstrom und dessen Nettoflussrichtung.

Aus den 1.600 Raumpunkten wurden 9 bzw. 25 ausgewählt, und zwar diejenigen Raumpunkte, die das Zentrum eines Kompartimentes bilden. Anhand der diesen Raumpunkten zugrunde liegenden Abklingreihen der Tracerkonzentration wurden die Luftvolumenströme ermittelt. Es werden dabei weder Einlass- oder Auslassgeschwindigkeiten berücksichtigt; die Lösung stellt sich durch Iteration von selbst ein. Die Werte für die Luftvolumenströme bei Anwendung des Kompartimentalisierungsverfahrens sind per Zufallsprinzip dem 13. Zeitschritt entnommen. Prinzipiell kann hierzu jeder beliebige Zeitschritt herangezogen werden. Die Anzahl der Zeitschritte, für die eine Lösung erzielt kann, ist dabei abhängig von der Länge der zugrunde liegenden, aufgenommen Zeitreihe. Die vollständige Auflistung aller Luftvolumenströme zu den Modellversuchen findet sich in Tabelle 7 im Anhang.

Es werden vier Simulationsbeispiele vorgestellt. Die Ergebnisse nach CFD stellen die exakte Lösung dar.

Beispiel 1: Raumbestückung mit 9 Sensoren

Beispiel 2: Raumbestückung mit 25 Sensoren

Beispiel 3: Raumbestückung mit 9 Sensoren und eingebautem Hindernis

Beispiel 4: Raumbestückung mit 9 Sensoren, versetzter Einlassöffnung und zwei Auslässen

Die Beispiele 1 und 2 beziehen sich auf das bereits behandelte Simulationsbeispiel in Kapitel 2.1.4. Sie werden zur Vervollständigung der dortigen Thematik gezeigt.

Abbildung 34 zeigt das Strömungsfeld und die Ergebnisse der Volumenstromberechnung bei einer Raumbestückung mit 9 Sensoren. Die Luft strömt oben links herein und unten rechts hinaus. Die 
Strömungsgeschwindigkeit ist an der Oberseite und rechten Wandseite am höchsten. Unten und links erfolgt die Rückströmung in abgeschwächter Form. In beiden Fällen erfolgt der Transport der Tracermoleküle durch Strömungsmitnahme. Die Volumenströme im Zentrum des Raumes sind sehr gering, hier findet hauptsächlich ein turbulenter Stoffaustausch statt. Der Strömungsverlauf und die Höhe des tatsächlichen Luftwechsels von $0,20 \mathrm{~m}^{3} / \mathrm{s}$ werden durch die Kompartimentalisierungsmethode richtig wiedergegeben. Die Werte der Luftwechselzahlen nach Abklingverfahren liegen um $30 \% \mathrm{zu}$ niedrig.

CFD-Simulation; $v_{\text {Einlass }}=1 \frac{m}{s}$

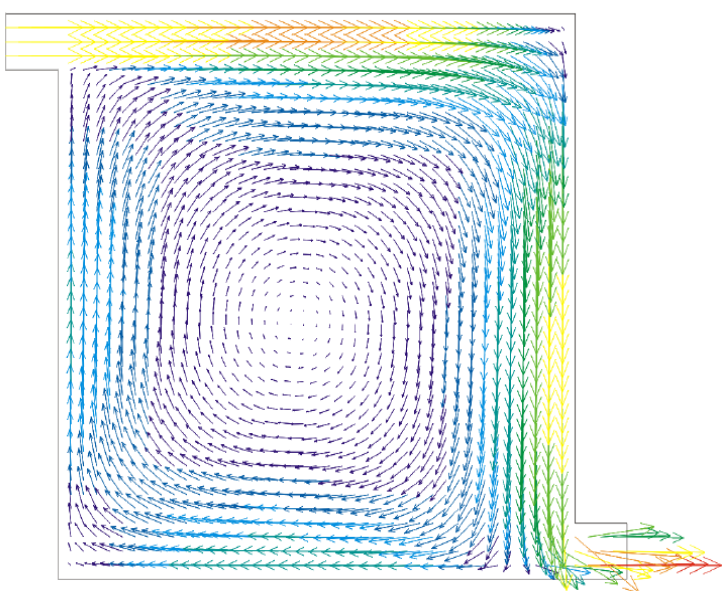

Kompartimentalisierungsmethode (13. Zeitschritt)

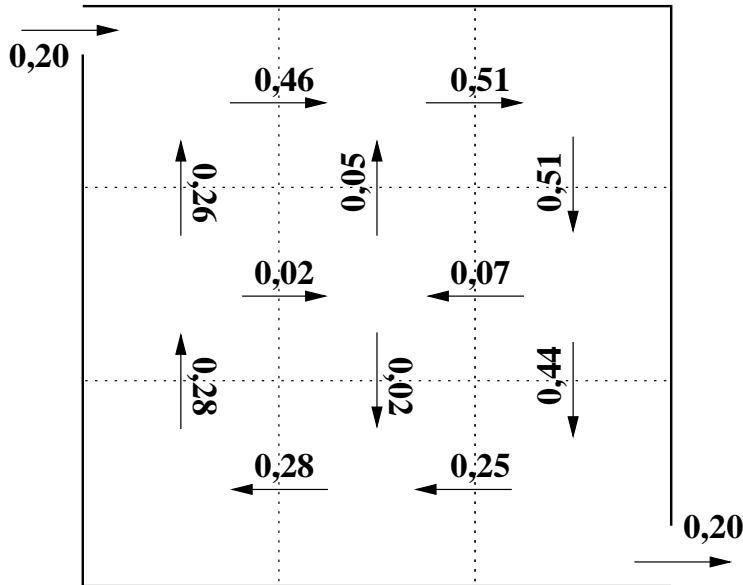

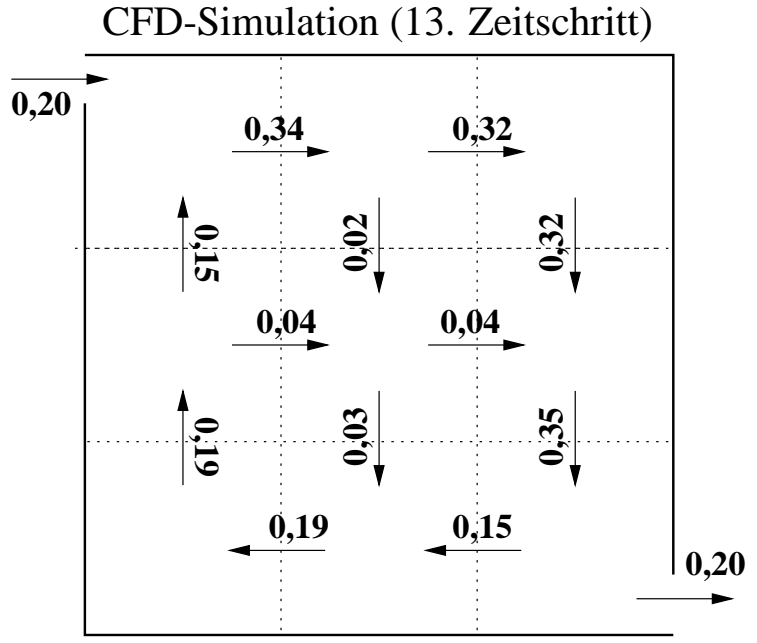

Abklingmethode (Mittel aller Abschnitte)

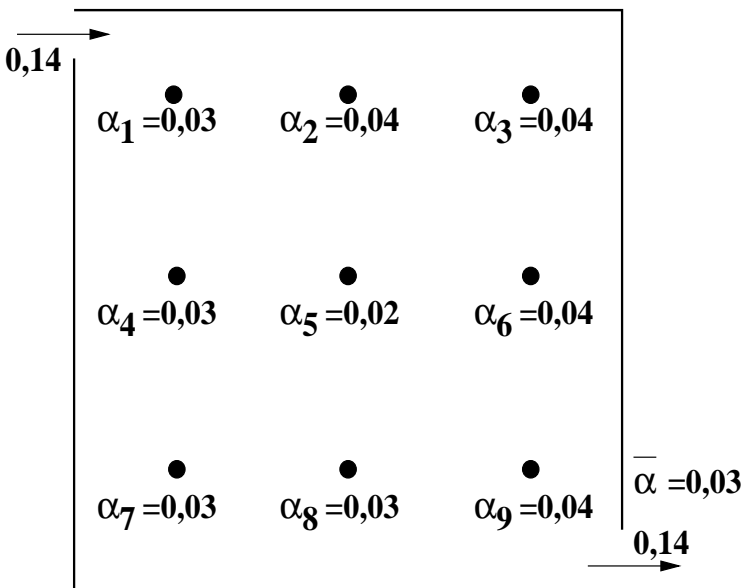

Abbildung 34: Geschwindigkeitsfeld bei der Durchströmung eines zweidimensionalen Raumquerschnittes $(2 \mathrm{~m} \times 2 \mathrm{~m})$ mit $v=1 \mathrm{~m} / \mathrm{s}$ im Einlass; $R e=13.000$. Der Betrag $\left[\mathrm{m}^{3} / \mathrm{s}\right]$ und die Richtung der errechneten Volumenströme bei Anwendung der Strömungssimulation sowie der Kompartimentalisierung (9 Kompartimente; 13. Zeitschritt) sind ausgewiesen. Die Einheit der gewonnenen mittleren Luftwechselzahlen $\alpha$ mittels Abklingmethode ist $s^{-1}$. 
Abbildung 35 zeigt das Strömungsfeld und die Ergebnisse der Volumenstromberechnung bei einer Raumbestückung mit 25 Sensoren. Das Strömungsfeld ist identisch zu Beispiel 1. Im Unterschied dazu stehen jetzt mehr Sensoren zur Verfügung. Der Strömungsverlauf wird durch die Kompartimentalisierungsmethode richtig wiedergegeben. Die Abweichungen zum tatsächlichen Luftwechsel von $0,20 \mathrm{~m}^{3} / \mathrm{s}$ betragen beim Kompartimenteverfahren rund $5 \%$ und beim Abklingverfahren $25 \%$.

CFD-Simulation; $v_{\text {Einlass }}=1 \frac{\mathrm{m}}{\mathrm{s}}$

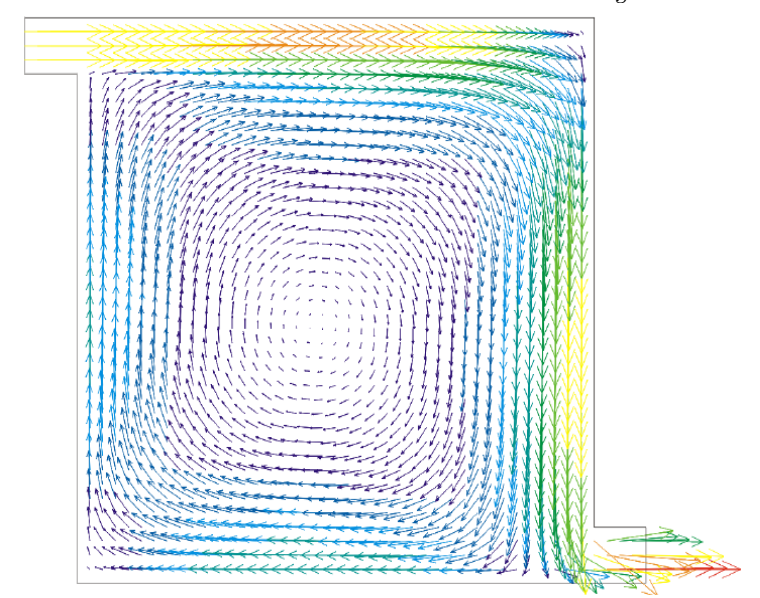

Kompartimentalisierungsmethode (13. Zeitschritt)

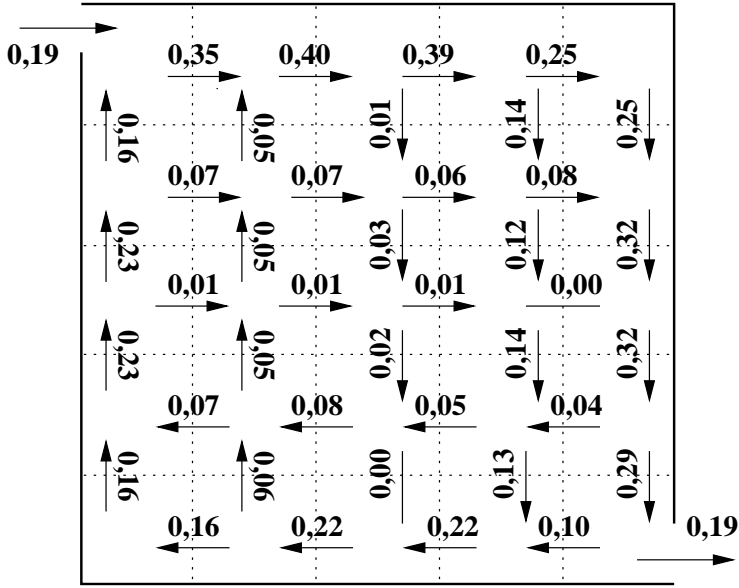

CFD-Simulation (13. Zeitschritt)

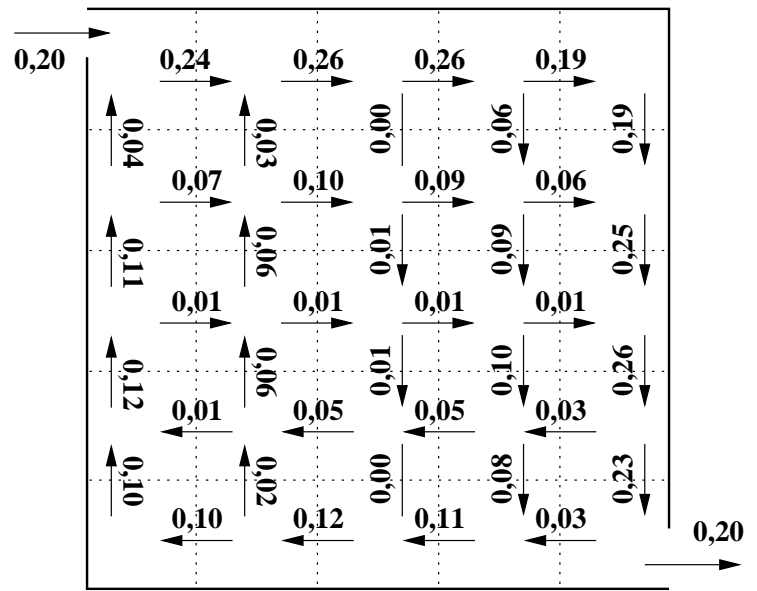

Abklingmethode (Mittel aller Abschnitte)

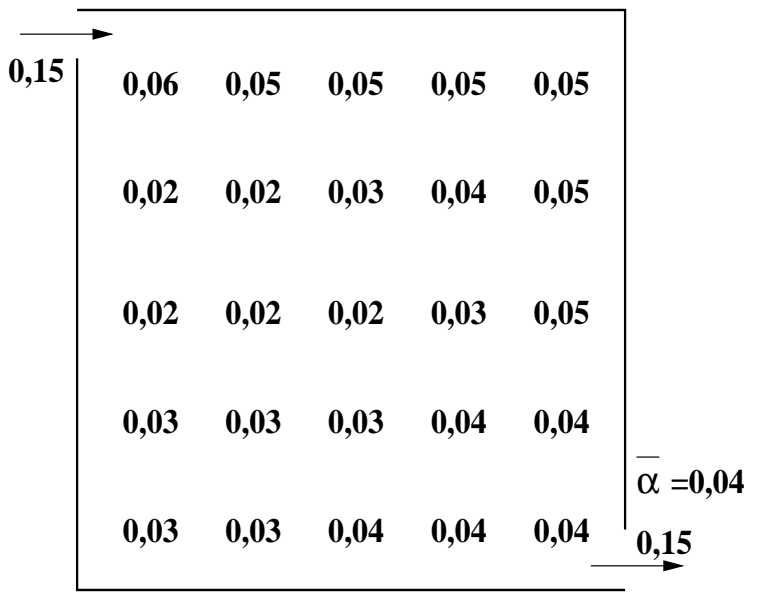

Abbildung 35: Geschwindigkeitsfeld bei der Durchströmung eines zweidimensionalen Raumquerschnittes $(2 \mathrm{~m} \times 2 \mathrm{~m})$ mit $v=1 \mathrm{~m} / \mathrm{s}$ im Einlass; $R e=13.000$. Der Betrag $\left[\mathrm{m}^{3} / \mathrm{s}\right]$ und die Richtung der errechneten Volumenströme bei Anwendung der Strömungssimulation sowie der Kompartimentalisierung (25 Kompartimente; 13. Zeitschritt) sind ausgewiesen. Die Einheit der gewonnenen mittleren Luftwechselzahlen $\alpha$ mittels Abklingmethode ist $s^{-1}$. 
Beispiel 3 zeigt das Strömungsfeld und die Ergebnisse der Volumenstromberechnung bei einer Raumbestückung mit 9 Sensoren. Zusätzlich ist ein Hindernis in Form einer undurchlässigen Abtrennung eingebaut. Der sich dadurch ergebene Strömungsverlauf ist in der linken oberen Grafik von Abbildung 36 zu erkennen. Die Kompartimentalisierungsmethode gibt den tatsächlichen Strömungsverlauf und Luftaustausch von 0, $20 \mathrm{~m}^{3} / \mathrm{s}$ korrekt wieder. Die Abweichung bei der Volumenstromberechnung beträgt bei der Anwendung der Abklingmethode in diesem Beispiel $40 \%$.

CFD-Simulation; $v_{\text {Einlass }}=1 \frac{\mathrm{m}}{\mathrm{s}}$

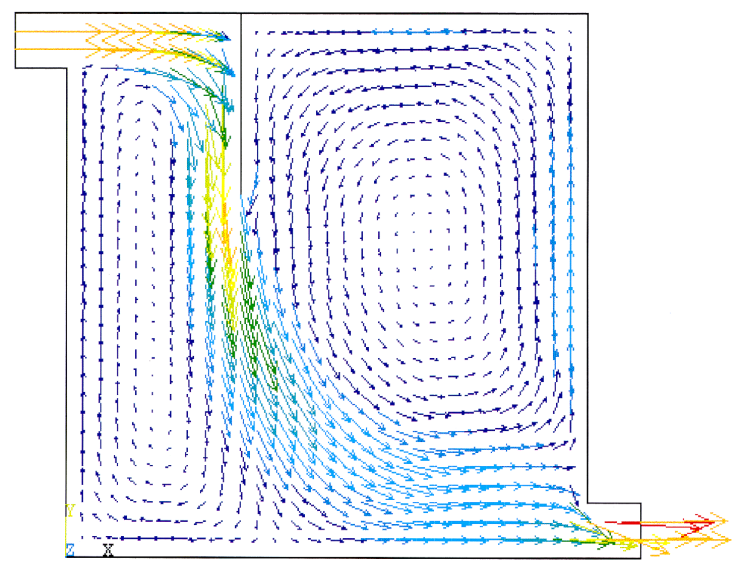

Kompartimentalisierungsmethode (13. Zeitschritt)

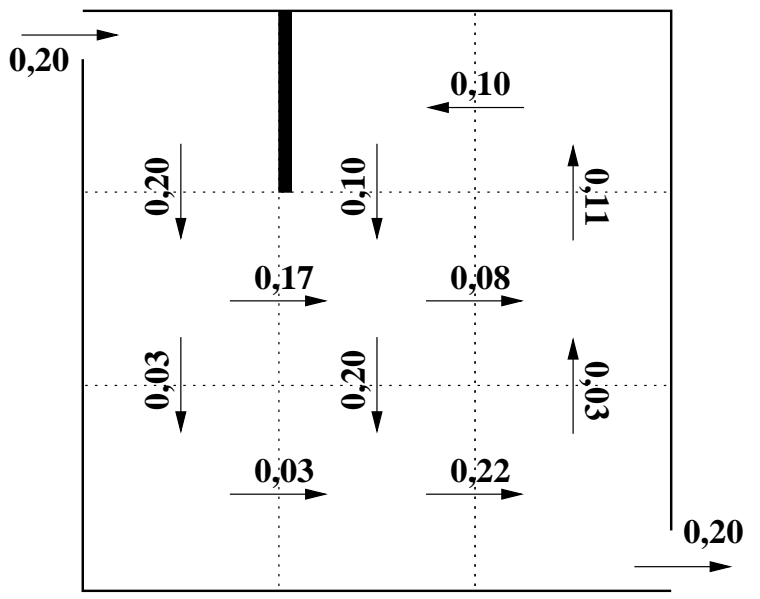

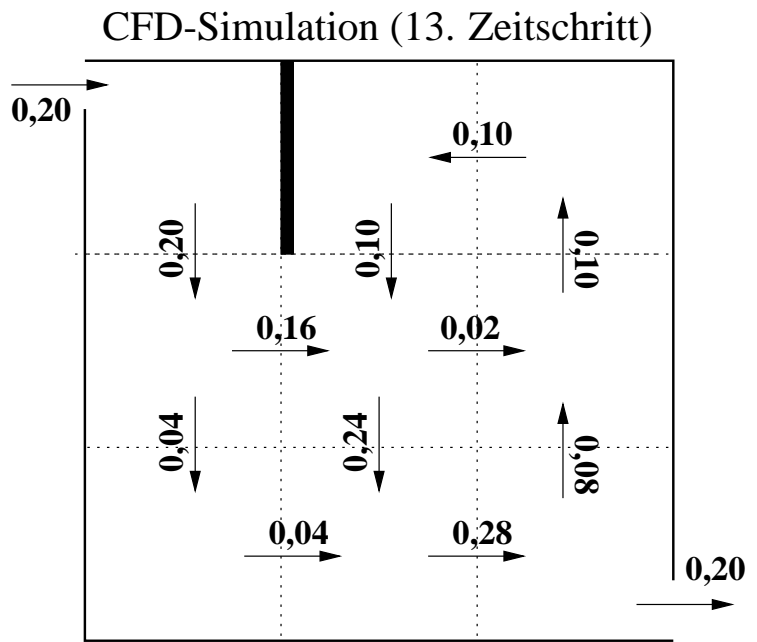

Abklingmethode (Mittel aller Abschnitte)

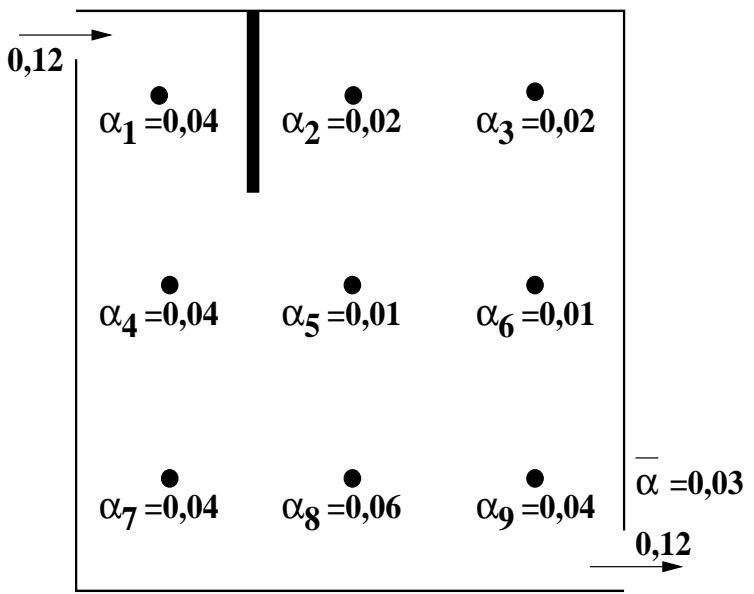

Abbildung 36: Geschwindigkeitsfeld bei der Durchströmung eines zweidimensionalen Raumquerschnittes ( $2 \mathrm{~m} \times 2 \mathrm{~m}$ mit eingebautem Hindernis) mit $v=1 \mathrm{~m} / \mathrm{s}$ im Einlass; $R e=13.000$. Der Betrag $\left[\mathrm{m}^{3} / \mathrm{s}\right]$ und die Richtung der errechneten Volumenströme bei Anwendung der Strömungssimulation sowie der Kompartimentalisierung (9 Kompartimente; 13. Zeitschritt) sind ausgewiesen. Die Einheit der gewonnenen mittleren Luftwechselzahlen $\alpha$ mittels Abklingmethode ist $s^{-1}$. 
Beispiel 4 (Abbildung 37) zeigt das Strömungsfeld und die Ergebnisse der Volumenstromberechnung bei einer Raumbestückung mit 9 Sensoren. Zusätzlich zur veränderten Lage der Einlassöffnung ist eine zweite Austrittsöffnung eingebaut.

Der Wirbel unmittelbar über der Einlassöffnung wird vom Kompartimenteverfahren nicht erkannt, da er nur eine kleinräumige Turbulenz verursacht bzw. innerhalb eines Kompartimentes auftritt. Es treten durch die zweite Auslassöffnung zwei lokale Volumenströme auf, welche mit der Kompartimentalisierungsmethode korrekt erfasst werden.

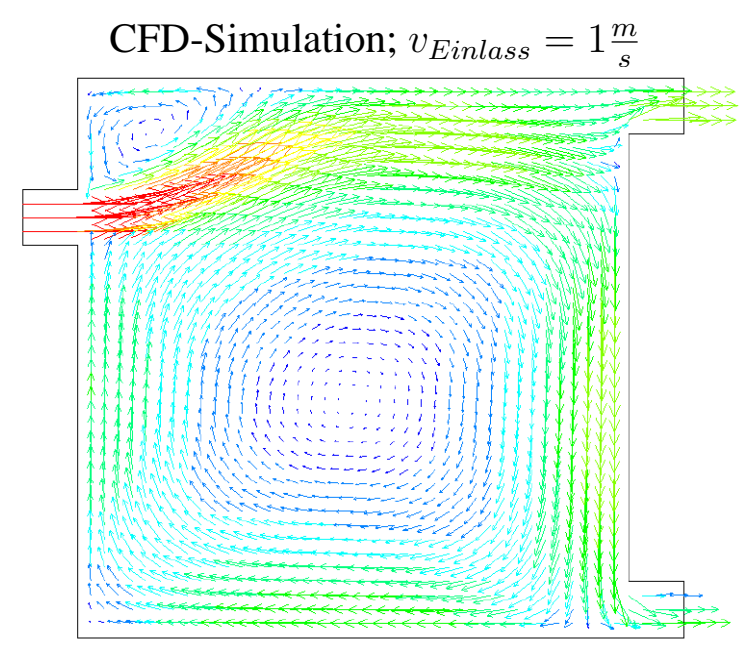

Kompartimentalisierungsmethode (13. Zeitschritt)

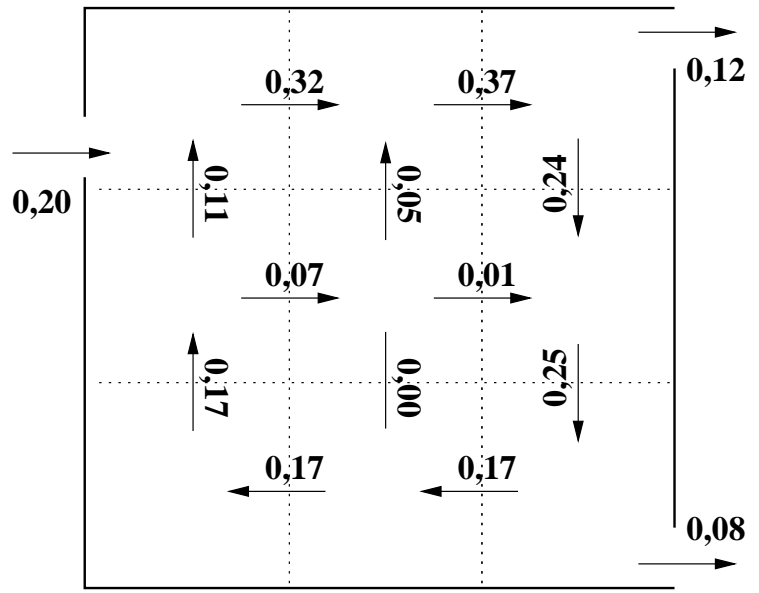

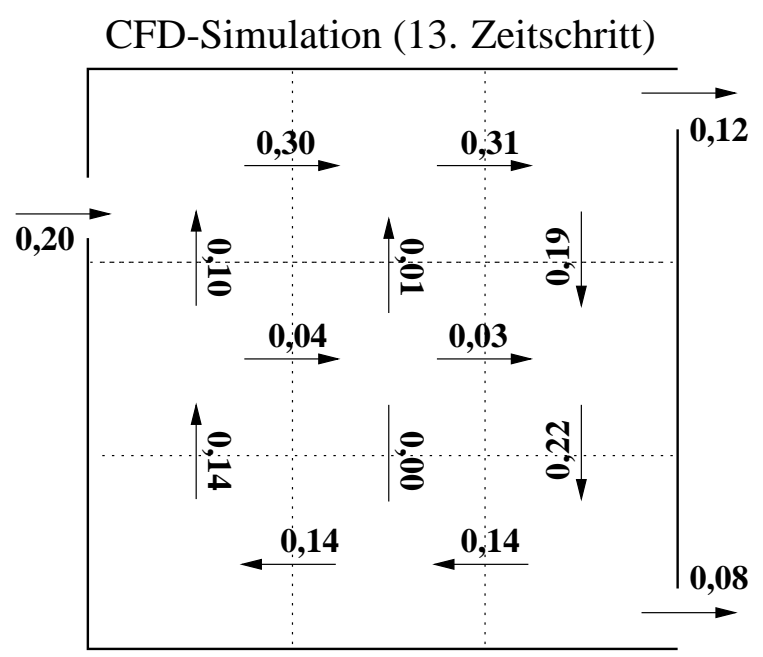

Abklingmethode (Mittel aller Abschnitte)

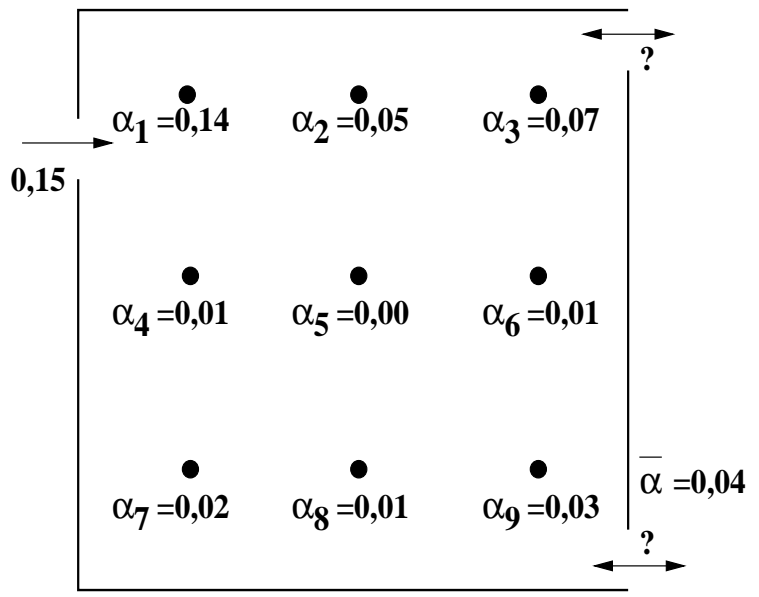

Abbildung 37: Geschwindigkeitsfeld bei der Durchströmung eines zweidimensionalen Raumquerschnittes ( $2 \mathrm{~m}$ x $2 \mathrm{~m}$; versetzte Einlassöffnung; zwei Auslässe) mit $v=1 \mathrm{~m} / \mathrm{s}$ im Einlass; $R e=13.000$. Der Betrag $\left[\mathrm{m}^{3} / \mathrm{s}\right]$ und die Richtung der errechneten Volumenströme bei Anwendung der Strömungssimulation sowie der Kompartimentalisierung (9 Kompartimente; 13. Zeitschritt) sind ausgewiesen. Die Einheit der gewonnenen mittleren Luftwechselzahlen $\alpha$ mittels Abklingmethode ist $s^{-1}$. 
Mittels Abklingverfahren kann die Strömungsrichtung und damit die Lokalität des Luftein- oder Austritts nicht festgestellt werden. Der Strömungsverlauf und die Höhe des tatsächlichen Luftwechsels von $0,20 \mathrm{~m}^{3} / \mathrm{s}$ werden durch die Kompartimentalisierungsmethode richtig wiedergegeben. Die Werte der Luftwechselzahlen nach Abklingverfahren liegen um $25 \%$ zu niedrig.

Tabelle 2: Ergebnisübersicht zu den systematischen Modellversuchen. Dargestellt ist jeweils die Höhe des Volumenstromes des 13. Zeitschritts bzw. der gemittelte Volumenstrom nach Abklingmethode.

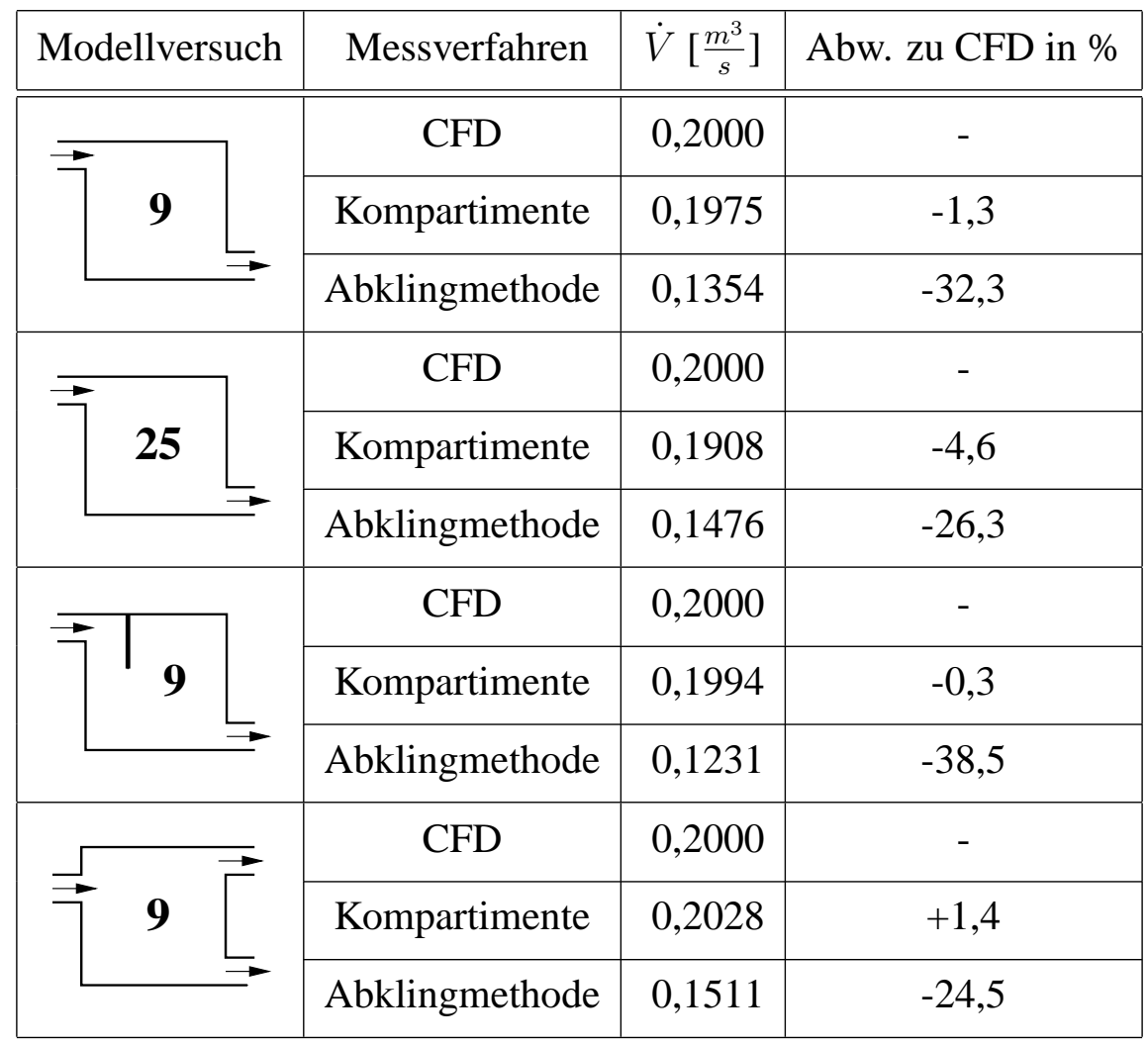

Tabelle 2 fasst die Ergebnisse der theoretischen Modellversuche zusammen. Das Ziel der Versuche ist es, das Kompartimente- und Abklingverfahren anhand numerischer Beispielsimulationen zu "kalibrieren". Hierzu wird das Softwarepaket ANSYS verwendet. Die Ergebnisse der CFD-Technik können aufgrund der idealisierten Versuchsdurchführung als exakte Lösungen angesehen werden.

Zunächst wird der 2 m x 2 m Raum mit 9 und anschließend 25 Sensoren bestückt. Der Effekt einer Sensorzahlerhöhung ist hiermit dargestellt. Das Kompartimenteverfahren liefert in beiden Fällen ein zufriedenstellendes Ergebnis, bereits bei einer Anzahl von 9 Sensoren wird der richtige Wert für den Volumenstrom ermittelt. Bei einer Bestückung mit 25 Sensoren treten mittels Kompartimentalisierungsmethode im weiteren Rechenverlauf die größten Abweichungen auf, die teilweise rund $10 \%$ betragen (siehe Tabelle 7 im Anhang). Die Zahl der unbekannten Austauschkoeffizienten beträgt in diesem Fall 85. Bei gleicher Länge der Zeitreihe, liegt die Zahl der Unbekannten im Fall einer 
Bestückung mit 9 Sensoren bei 26. Damit können mehr Zeitschritte für die Berechnung der Austauschkoeffizienten zusammengefasst werden, welches zu einer Genauigkeitserhöhung führt (Kapitel 4.3.2). Die Ergebnisse für das Abklingverfahren wurden bereits in Abbildung 8 vorgestellt: sie weisen zu geringe Werte auf. Das Strömungsbild kann unter Umständen anhand der Lage des Messpunktes und des Betrages der einzelnen Luftwechselkoeffizienten "erahnt" werden. Ermöglicht wird dies mit hoher Wahrscheinlichkeit durch die vorliegende vollständige Durchmischung des Tracers mit der Raumluft.

Im dritten Fall wird ein Hindernis eingebaut. Dadurch kommt es zu einer Änderung des Strömungsfeldes. Das Abklingverfahren weist in diesem Fall mit $40 \%$ die höchste Fehlerquote auf. Die Ergebnisse mittels Kompartimentalisierungsmethode stimmen mit der exakten Lösung gut überein.

Im vierten Fall wird eine zweite Auslassöffnung eingebracht, zudem ist die Einlassöffnung versetzt. Sie liegt jetzt in Höhe des ersten Sensors, so dass nach Abklingmethode auch ein erhöhter Luftwechsel für diesen Raumpunkt festgestellt werden kann. Eine Aussage zum eindeutigen Luftaustritt an beiden Auslässen kann nicht getroffen werden. Dies ist mit der Kompartimentalisierungsmethode möglich, die Öffnungen sind eindeutig einem Sensor bzw. einem Kompartiment zuzuordnen. Der tatsächliche Luftwechsel wird mit einer geringfügigen Abweichung richtig wiedergegeben, die Ergebnisse nach Abklingmethode liegen wiederum zu niedrig.

Im Durchschnitt über alle theoretischen Modellversuche ergeben sich bei Anwendung der Kompartimentalisierungsmethode Abweichungen von ca. $2 \%$, bei Anwendung des Abklingverfahrens Abweichungen von etwa $30 \%$. 


\subsection{Feldversuche}

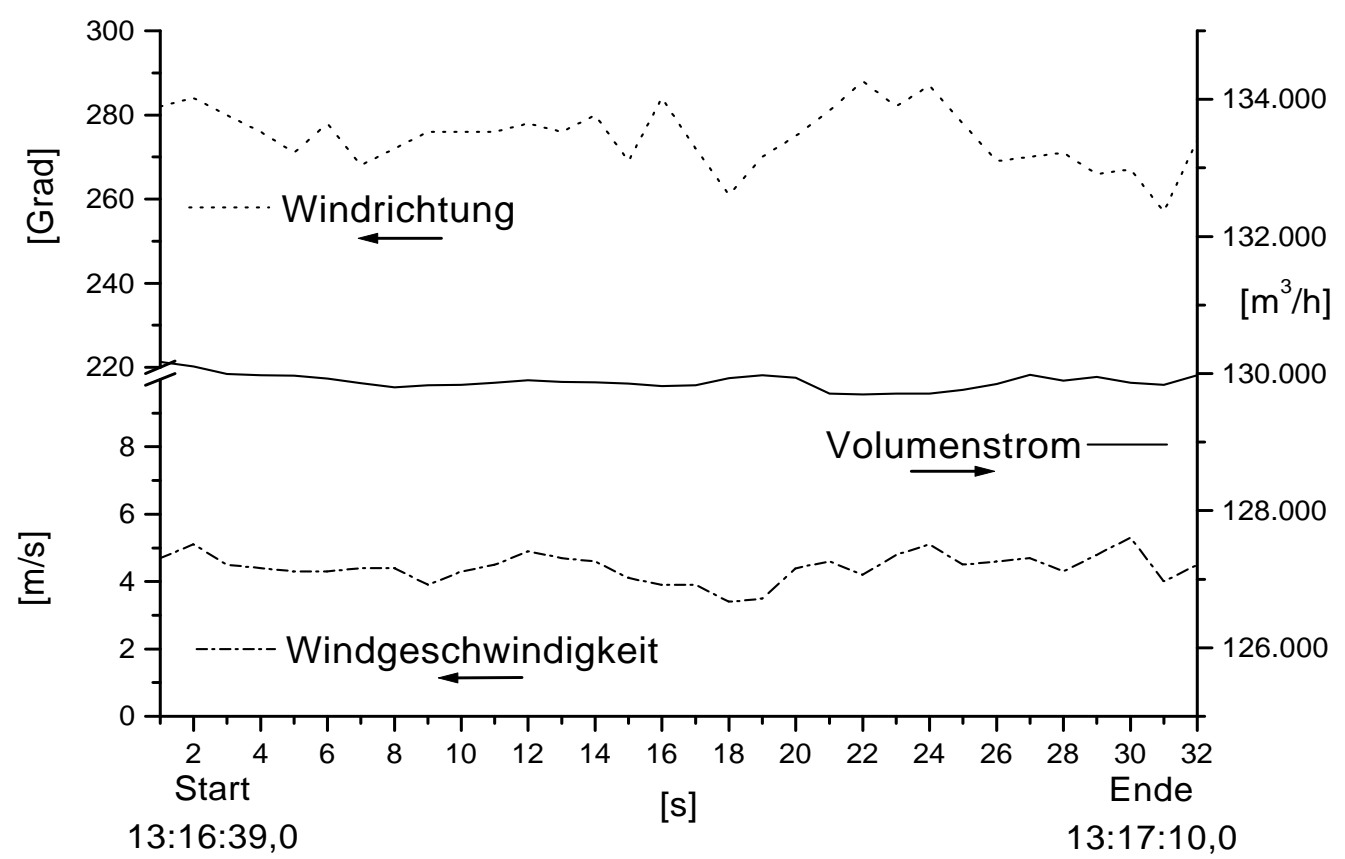

Abbildung 38: Verlauf des Luftvolumenstromes, der Windgeschwindigkeit und Windrichtung über einen betrachteten Zeitraum von 32 Sekunden im Stall Faulenhorst.

Abbildung 38 zeigt den Verlauf des Volumenstromes im Beispiel Faulenhorst. Von geringen Schwankungen abgesehen ist dieser über den gesamten Zeitabschnitt konstant. Er liegt im Durchschnitt bei $130.000 \mathrm{~m}^{3} / \mathrm{h}$. Bei einem Stallvolumen von $3.045 \mathrm{~m}^{3}$ entspricht das einem Luftwechsel von durchschnittlich $43 / h$. Durch die weit auseinander liegenden Austauschflächen (Tore in Abbildung 28) machen sich die zeitweiligen Änderungen in der Windrichtung und Windgeschwindigkeit nur in geringem Umfang auf den Luftdurchsatz bemerkbar.

Die Ergebnisse der Volumenstromberechnung sind stellvertretend für den 13. Zeitschritt in Abbildung 39 ersichtlich. Dargestellt sind die Höhe der lokalen Volumenströme und deren Flussrichtung (oben). In der Mitte wird aus Gründen der Übersicht das reine Strömungsfeld gezeigt. In der unteren Darstellung wird versucht, den wahrscheinlichen Strömungsverlauf im Stall Faulenhorst anhand der Höhe und Richtung der errechneten Austauschkoeffizienten nachzufahren. Die Hauptluftströmung tritt in den zur Windanströmrichtung ausgerichteten Toren ein und strömt hauptrangig zum Monoschacht hinaus. Die von der Luftströmung direkt angeströmte Stallhälfte ist durch Wirbel gekennzeichnet, es treten hier zahlreiche Turbulenzen auf. Stallmittig ist eine Kurzschlussströmung vom Melkstand zum Nachbarstall vorhanden, die zudem den Bereich um den Luftschacht sowie die von der Windanströmrichtung indirekt betroffene Stallhälfte erfasst. 


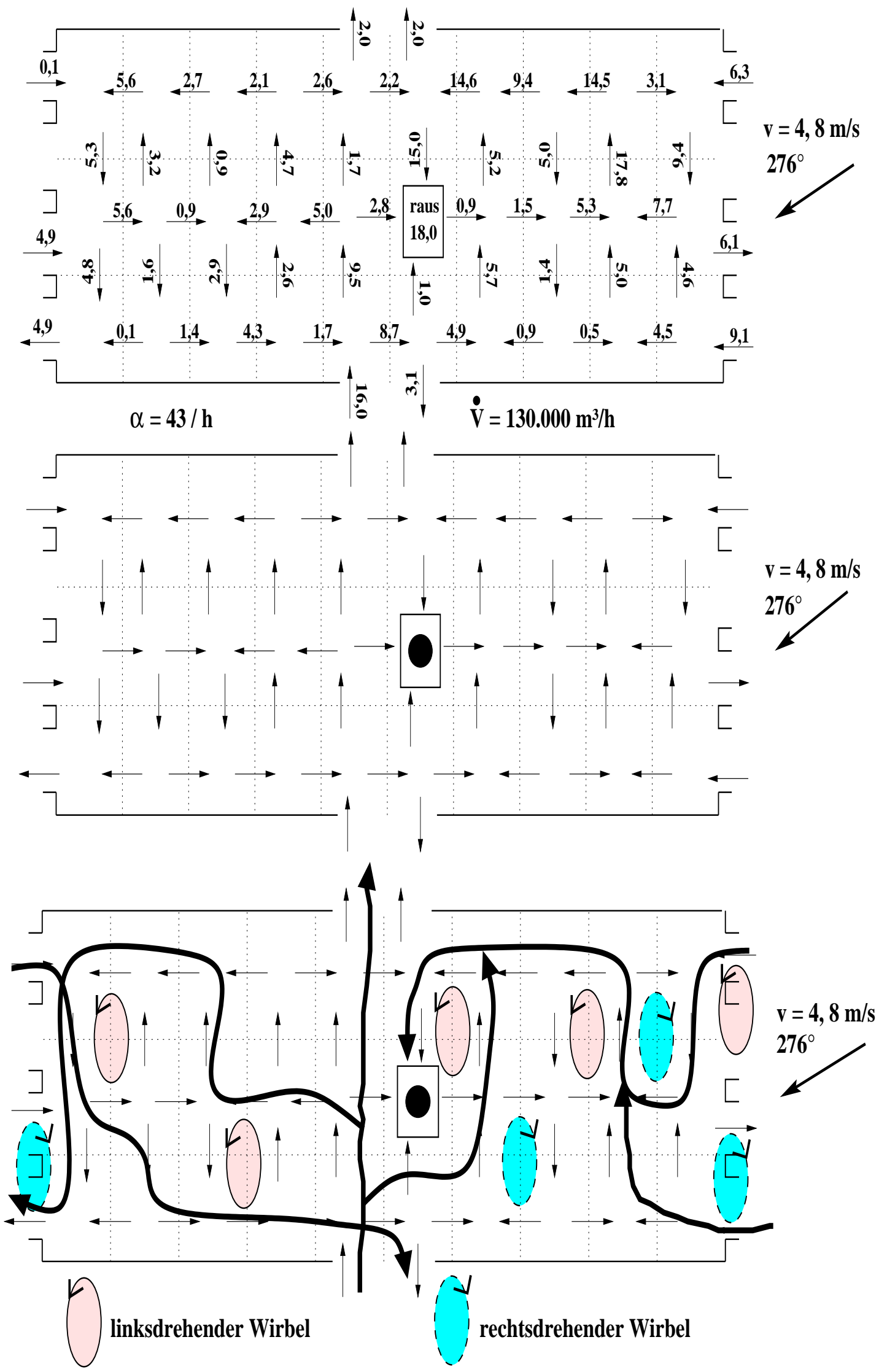

Abbildung 39: Betrag (oben) und Richtung (mittig) der errechneten Austauschkoeffizienten (13. Zeitschritt) nach der Kompartimentalisierungsmethode sowie der wahrscheinliche Strömungsverlauf (unten) im Stall Faulenhorst bei einer Windanströmgeschwindigkeit von $4,8 \mathrm{~m} / \mathrm{s}$ ( $2 \mathrm{~m}$ Höhe). Rechts- und linksdrehende Wirbel sind eingezeichnet. 


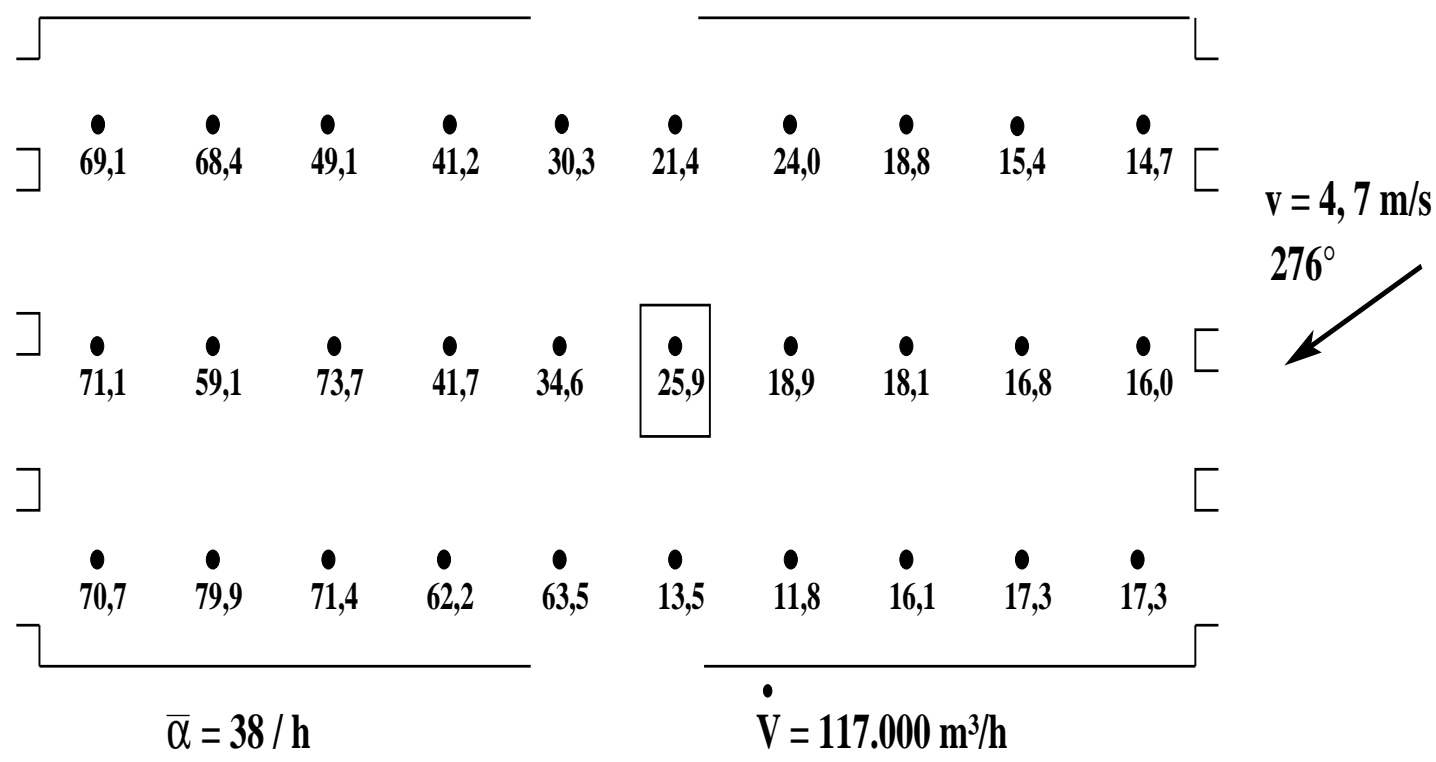

Abbildung 40: Mittlere Luftwechselkoeffizienten aller Sensoren sowie Höhe des mittleren Gesamtluftwechsels $\bar{\alpha}$ im Stall Faulenhorst.

In Abbildung 40 sind die Höhe der errechneten mittleren Luftwechselkoeffizienten und der Gesamtluftwechsel nach der Abklingmethode eingezeichnet. Letzterer liegt um etwa $10 \%$ niedriger als der Wert nach Kompartimentalisierungsmethode ( $\alpha=43 \Rightarrow$ Absolutwert). Abbildung 40 besagt, dass in der linken Stallhälfte deutlich höhere Luftaustauschraten vorzufinden sind als in der rechten, der Windanströmrichtung zugewandten Seite. Vergleichende Aussagen zum lokalen Luftwechsel sind aber in Wirklichkeit nicht möglich, da die Mittelwertbildung der einzelnen Luftwechselkoeffizienten aufgrund der zu verschiedenen Zeitpunkten einsetzenden Konzentrationsmaxima auch zu unterschiedlichen Zeitpunkten vorgenommen wurde. Im Unterschied zu den theoretischen Modellversuchen liegt in der Praxis keine homogene Traceranfangskonzentration vor. Anhand der Höhe der Luftwechselkoeffizienten an den einzelnen Sensoren können damit keine Aussagen zum etwaigen Verlauf der Strömung getroffen werden.

In Tabelle 3 sind die durchschnittlich gemessenen Konzentrationen, in Tabelle 4 die durchschnittlichen Massenausträge der untersuchten Gase aufgelistet. Die vollständige Auflistung aller Luftvolumenströme für den Stall Faulenhorst findet sich in Tabelle 8 im Anhang. 
Tabelle 3: Durchschnittliche Konzentration $\left[\frac{\mathrm{mg}}{\mathrm{m}^{3}}\right]$ bzw. $[\mathrm{ppm}]$ von Ammoniak, Methan, Lachgas, Kohlendioxid und Wasserdampf ermittelt über 9 Messpunkte über einen Zeitraum von 32 Sekunden (Stall Faulenhorst).

\begin{tabular}{|c|c|c|}
\hline $\mathrm{Gas}$ & $\bar{C}\left[\frac{m g}{m^{3}}\right]$ & $\bar{C}[\mathrm{ppm}]$ \\
\hline \hline $\mathrm{NH}_{3}$ & 4 & 6 \\
\hline $\mathrm{CH}_{4}$ & 38 & 58 \\
\hline $\mathrm{N}_{2} \mathrm{O}$ & 0,2 & 0,1 \\
\hline $\mathrm{CO}_{2}$ & 1.997 & 1.109 \\
\hline $\mathrm{H}_{2} \mathrm{O}$ & 10.846 & 14.716 \\
\hline
\end{tabular}

Tabelle 4: Errechnete Massenströme $\left[\frac{g}{G V \cdot h}\right]$ für Ammoniak, Methan, Lachgas, Kohlendioxid und Wasserdampf nach der Kompartimentalisierungsmethode (13. Zeitschritt) bzw. nach der Abklingmethode (Mittelwert aus Zeitschritt 1 bis 32) im Stall Faulenhorst.

\begin{tabular}{|c|c|c|}
\hline $\mathrm{Gas}$ & Kompartimentimentalisierungsmethode & Abklingmethode \\
\hline \hline $\mathrm{NH}_{3}$ & 3 & 3 \\
\hline $\mathrm{CH}_{4}$ & 31 & 28 \\
\hline $\mathrm{N}_{2} \mathrm{O}$ & 0,2 & 0,1 \\
\hline $\mathrm{CO}_{2}$ & 1.600 & 1.440 \\
\hline $\mathrm{H}_{2} \mathrm{O}$ & 8.700 & 7.800 \\
\hline
\end{tabular}

Abbildung 41 gibt einen Überblick über den Verlauf der Massenströme im Stall Faulenhorst mittels Kompartimentalisierungsverfahren. Durch den annähernd unveränderten Volumenstrom ergibt sich zwangsläufig ein ebenso konstanter Verlauf der Massenausträge. Die Verläufe stellen keine Tagesgänge dar, sondern resultieren aus einer Spotmessung. Einflüsse durch Tieraktivitäten etc. bleiben somit unberücksichtigt. Hierzu müssen Luftwechseluntersuchungen über längere Zeiträume durchgeführt werden. 

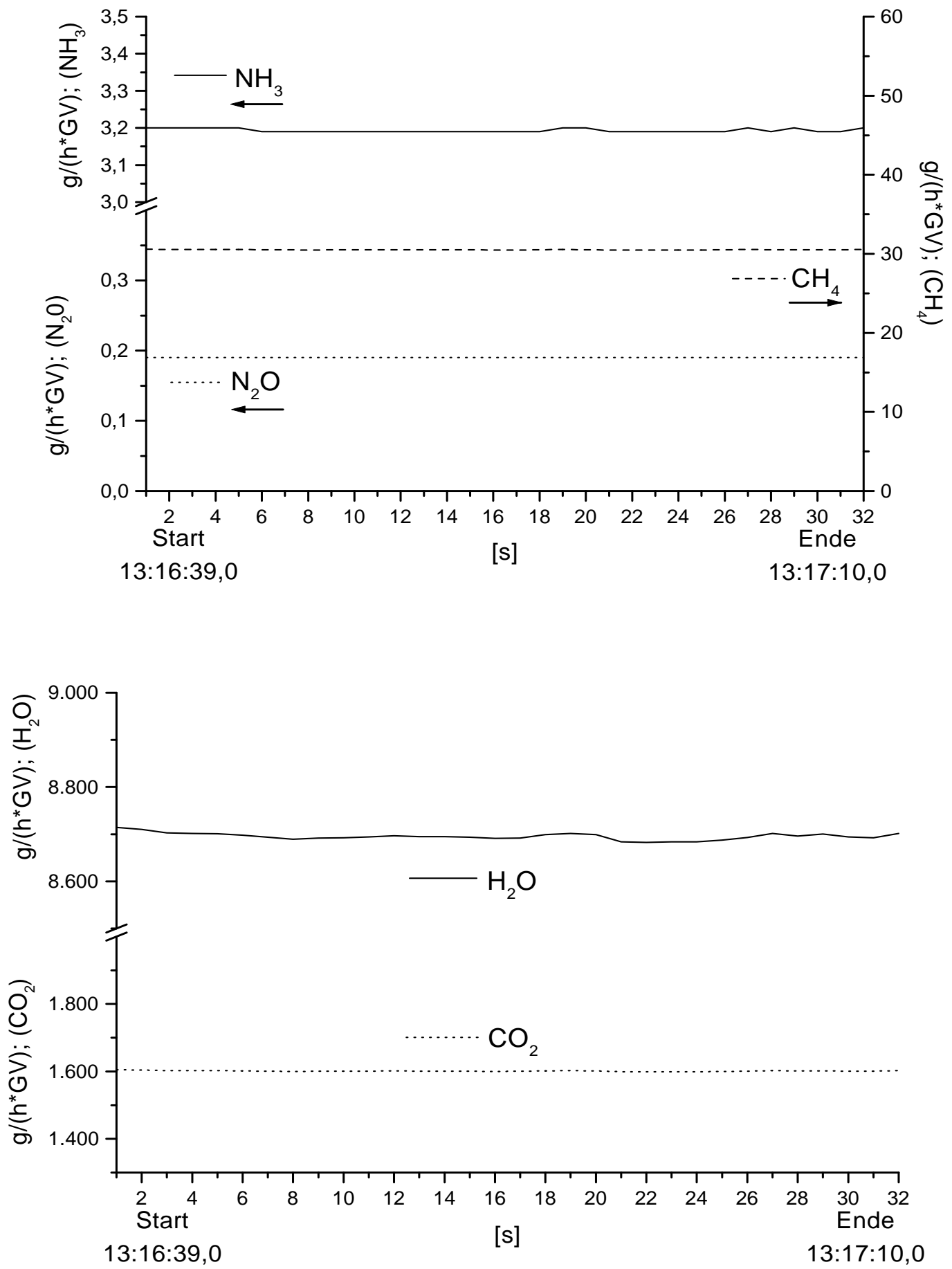

Abbildung 41: Verlauf der Massenströme für Ammoniak, Methan und Lachgas (oben) sowie von Kohlendioxid und Wasserdampf (unten) ermittelt nach der Kompartimentalisierungsmethode über einen betrachteten Zeitraum von 32 Sekunden (Stall Faulenhorst). 


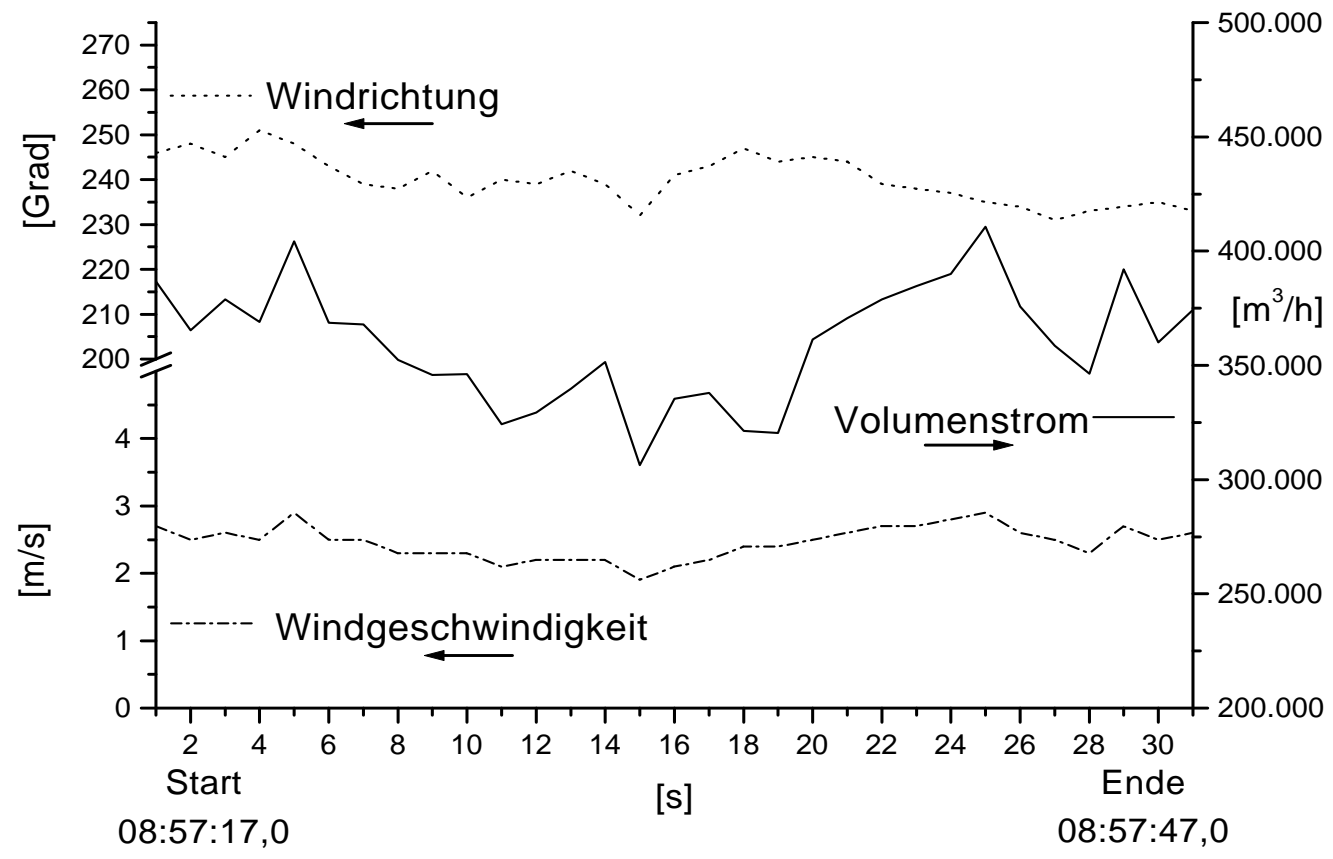

Abbildung 42: Verlauf des Luftvolumenstromes, der Windgeschwindigkeit und Windrichtung über einen betrachteten Zeitraum von 31 Sekunden im Stall Rothenstadt.

Abbildung 42 zeigt den Verlauf des Volumenstromes im Beispiel Rothenstadt. Er ist in diesem Offenfrontstall wesentlich strukturierter ausgeprägt als im zuvor behandelten Offenstall mit Lüftungsschacht. Hervorgerufen wird dies durch die vorhandenen, großflächigen Austauschflächen des Stalles mit der Umgebung. Bereits kleine Änderungen der Windgeschwindigkeit und der Windrichtung führen zu einer sofortigen Reaktion des Systems. Der Volumenstrom liegt durchschnittlich bei $360.000 \mathrm{~m}^{3} / \mathrm{h}$. Bei einem Stallvolumen von $3.411 \mathrm{~m}^{3}$ entspricht dies einem mittlerem Luftwechsel von $106 / h$.

Die Ergebnisse der Volumenstromberechnung sind stellvertretend für den 13. Zeitschritt in Abbildung 43 ersichtlich. Dargestellt sind die Höhe der lokalen Volumenströme und deren Flussrichtung (oben). In der Mitte wird aus Gründen der Übersicht das reine Strömungsfeld gezeigt. In der unteren Darstellung ist der wahrscheinliche Strömungsverlauf im Stall Rothenstadt dargestellt. Er ergibt sich aufgrund der Höhe und Richtung der errechneten Austauschkoeffizienten. Die Werte einiger Austauschkoeffizienten sind nicht angegeben, da der verwendete Algorithmus in diesem Fall keine Lösung liefert. Nähere Ausführungen dazu erfolgen in Kapitel 7. Die Hauptluftströmung trifft auf die westliche (rechte) Giebelseite. Durch die Auslegung als Spaceboard gelangen die Luftmassen in den Stall. Die stärksten Turbulenzen treten in den Kompartimenten 14 und 15 auf. Die dortigen Strömungen vereinen sich mit den durch die Rückseite eintretenden Luftmassen und strömen durch die offene Längsseite hinaus. Im Sheddach kommt es sowohl zu einem Luftein- als auch Luftaustritt. 

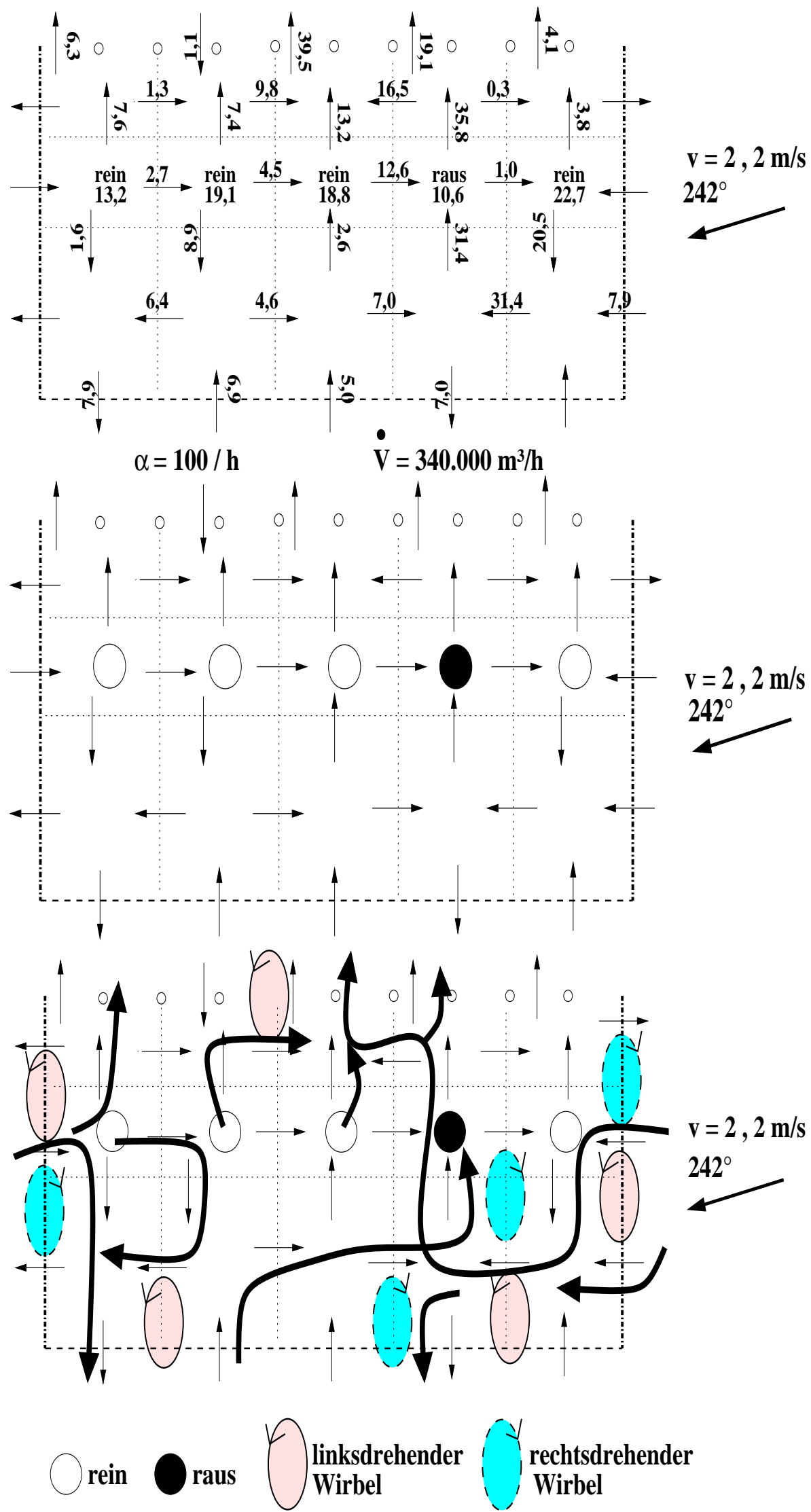

Abbildung 43: Betrag (oben) und Richtung (mittig) der errechneten Austauschkoeffizienten nach Kompartimentalisierungsmethode (13. Zeitschritt) sowie der wahrscheinliche Strömungsverlauf (unten) im Stall Rothenstadt bei einer Windanströmgeschwindigkeit von $2,2 \mathrm{~m} / \mathrm{s}$ (2 $\mathrm{m}$ Höhe). Rechts- und linksdrehende Wirbel sind eingezeichnet. 


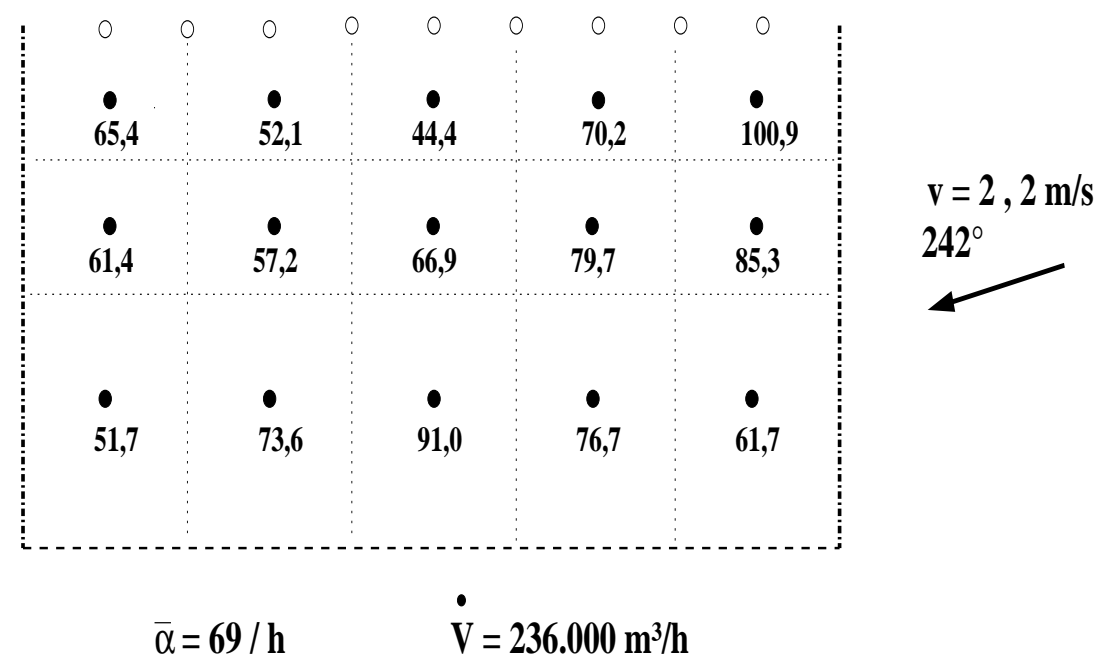

Abbildung 44: Mittlere Luftwechselkoeffizienten aller Sensoren sowie Höhe des mittleren Gesamtluftwechsels $\bar{\alpha}$ im Stall Rothenstadt.

In Abbildung 44 sind die errechneten mittleren Luftwechselkoeffizienten und der Gesamtluftwechsel $\bar{\alpha}$ nach der Abklingmethode dargestellt. Auch in diesem Fall sind aufgrund des zeitlich versetzten Auftretens der Tracerkonzentrationsmaxima keine vergleichenden Aussagen zum lokalen Luftwechsel möglich. Der Wert des ermittelten Luftvolumenstroms liegt um knapp 35 \% niedriger als der Wert nach Kompartimentalisierungsmethode ( $\alpha=100 \Rightarrow$ Absolutwert). Die vollständige Auflistung aller Luftvolumenströme mittels Kompartimentalisierungsmethode für den Stall Rothenstadt findet sich in Tabelle 8 im Anhang. Im Durchschnitt der beiden Praxisversuche ergibt sich bei Anwendung des Abklingverfahrens somit eine Differenz in Höhe von 22, $5 \%$.

In Tabelle 3 sind die gemessenen durchschnittlichen Konzentrationen der untersuchten Gase, in Tabelle 6 die durchschnittlichen Massenausträge der untersuchten Gase aufgelistet. Hierbei ist festzustellen, dass die gemessene $\mathrm{NH}_{3}$-Konzentration in Rothenstadt an der untersten Grenze des Nachweisbereiches liegt, der mit der eingesetzten Konzentrationsmesstechnik noch detektierbar ist. Die im Stall ermittelte Ammoniakkonzentration ist damit als Hintergrundkonzentration zu interpretieren. Die Lachgaskonzentration des untersuchten Offenfrontstalles liegt deutlich über der des Offenstalles mit Luftschacht in Faulenhorst. Die Werte für Methan, Kohlendioxid und Wasserdampf hingegen weisen niedrigere Werte auf. 
Tabelle 5: Durchschnittliche Konzentration $\left[\frac{\mathrm{mg}}{\mathrm{m}^{3}}\right]$ bzw. $[\mathrm{ppm}]$ von Ammoniak, Methan, Lachgas, Kohlendioxid und Wasserdampf ermittelt über 6 Messpunkte über einen Zeitraum von 31 Sekunden (Stall Rothenstadt).

\begin{tabular}{|c|c|c|}
\hline Gas & $\bar{C}\left[\frac{m g}{m^{3}}\right]$ & $\bar{C}[p p m]$ \\
\hline \hline $\mathrm{NH}_{3}$ & 0,1 & 0,1 \\
\hline $\mathrm{CH}_{4}$ & 3 & 5 \\
\hline $\mathrm{N}_{2} \mathrm{O}$ & 0,5 & 0,3 \\
\hline $\mathrm{CO}_{2}$ & 793 & 441 \\
\hline $\mathrm{H}_{2} \mathrm{O}$ & 4.823 & 6.544 \\
\hline
\end{tabular}

Tabelle 6: Errechnete Massenströme $\left[\frac{g}{G V \cdot h}\right]$ für Ammoniak, Methan, Lachgas, Kohlendioxid und Wasserdampf nach der Kompartimentalisierungsmethode (13. Zeitschritt) bzw. nach der Abklingmethode (Mittelwert aus Zeitschritt 1 bis 31) im Stall Rothenstadt.

\begin{tabular}{|c|c|c|}
\hline $\mathrm{Gas}$ & Kompartimentimentalisierungsmethode & Abklingmethode \\
\hline \hline $\mathrm{NH}_{3}$ & 0,9 & 0,5 \\
\hline $\mathrm{CH}_{4}$ & 24 & 17 \\
\hline $\mathrm{N}_{2} \mathrm{O}$ & 4 & 3 \\
\hline $\mathrm{CO}_{2}$ & 5.750 & 4.000 \\
\hline $\mathrm{H}_{2} \mathrm{O}$ & 35.000 & 24.300 \\
\hline
\end{tabular}

Abbildung 45 gibt einen Überblick über den Verlauf der Massenströme im Stall Rothenstadt mittels Kompartimenteverfahren. Der zeitlichen Schwankungen unterliegende Volumenstrom führt zu zeitlich unterschiedlich ausgeprägten Massenausträgen. Die Verläufe stellen auch in diesem Fall keine Tagesgänge dar, sondern resultieren aus einer Spotmessung. Durch die sehr geringe Ammoniakkonzentration in der Stallluft werden aus einem Offenfrontstall mit Milchkühen folglich nur geringfügige $\mathrm{NH}_{3}$-Emissionsmassenströme emittiert. 

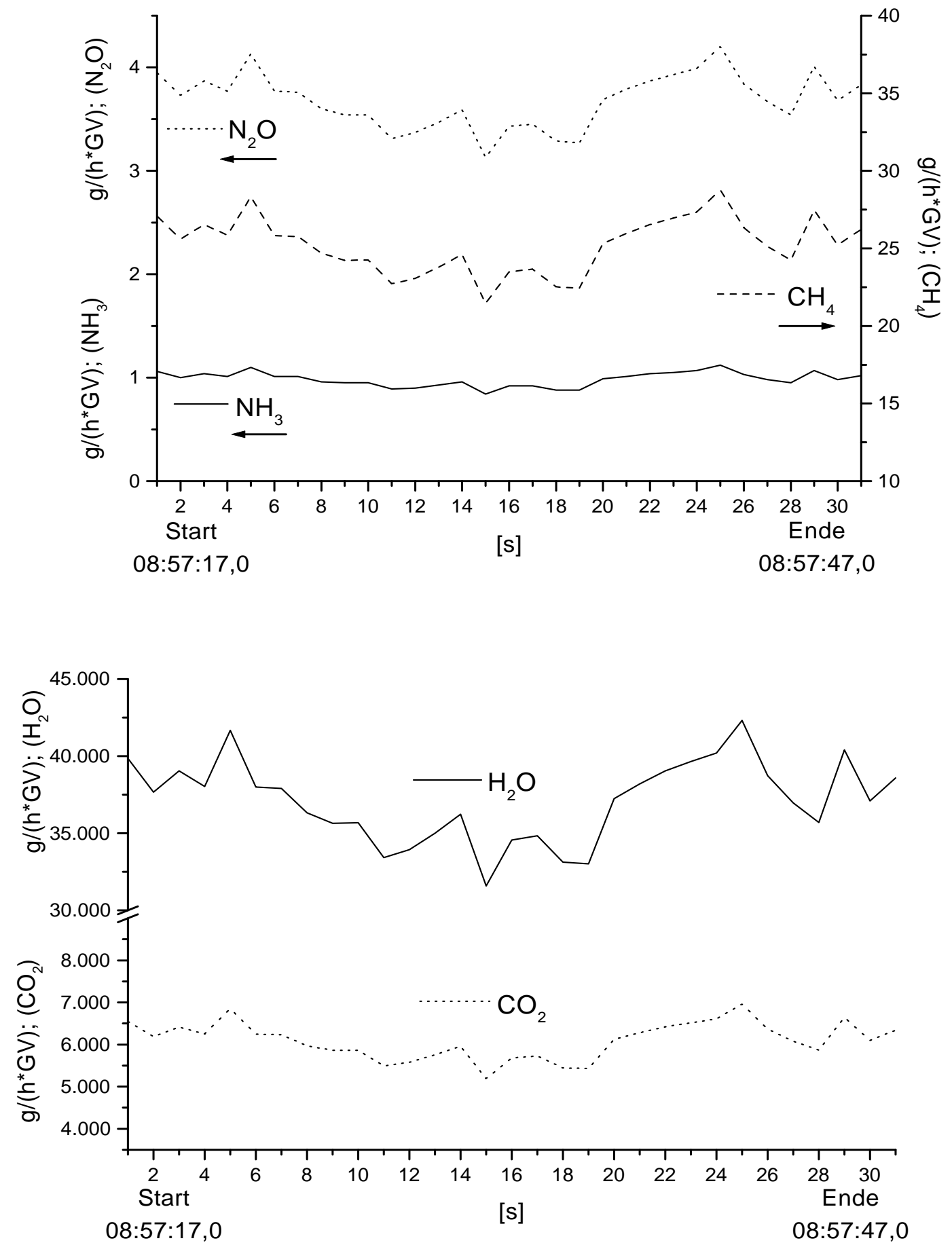

Abbildung 45: Verlauf der Massenströme für Ammoniak, Methan und Lachgas (oben) sowie von Kohlendioxid und Wasserdampf (unten) nach der Kompartimentalisierungsmethode über einen betrachteten Zeitraum von 31 Sekunden (Stall Rothenstadt). 


\section{Diskussion}

Ein Stall stellt in der freien atmosphärischen Strömung ein um- und/oder durchströmtes Hindernis dar. Damit lässt er sich mit den Gesetzen der Strömungsmechanik beschreiben. Eines der Hauptprobleme ist dabei die Größe des Hindernisses Stall, da dieser hinsichtlich der messtechnischen Aufzeichnung von Tracerkonzentrationen nur eingeschränkt erfassbar ist. Hier muss eine sehr deutliche Differenzierung zu konventionellen Tracermessungen in Bürogebäuden etc. gezogen werden. Die prinzipiellen physikalischen und strömungsmechanischen Gesetzmäßigkeiten gelten zwar in beiden Fällen, es ist jedoch ein erheblicher Unterschied, ob eine Luftwechselmessung in einem Büroraum $\left(V=50 \mathrm{~m}^{3}\right)$ oder in einem Stallgebäude $\left(V=5.000 \mathrm{~m}^{3}\right)$ durchgeführt wird.

Vergleicht man wiederum die im Rahmen der Arbeit errechneten durchschnittlichen Kompartimentsgrößen (ca. $100-230 \mathrm{~m}^{3}$ ) mit denen, die in der Meteorologie üblich sind ( $\mathrm{km}^{3}$ !), so stellen die Stallkontrollvolumina relativ kleine Raumelemente dar. Mit zunehmender Größe eines Kompartimentes werden Aussagen zum kompartimentsinternen Strömungsverlauf unsicherer. Es treten unkontrollierte Flüsse im Kompartiment auf [74], wie das z.B. in Abbildung 37 oben links der Fall ist. Dennoch: Die Bilanz über ein Kompartiment - welcher Größe auch immer - muss stets ausgeglichen sein.

In größeren Kompartimenten existieren Bereiche geringerer Lüftungseffektivität, der Austausch der Tracermoleküle erfolgt, im Vergleich zu den übrigen Kompartimentsbereichen, langsam. Damit erhöht sich gleichzeitig der Diffusionseinfluss. Da Strömungen in Ställen aufgrund der geringen Viskosität des Transportmediums Luft sowie durch die atmosphärisch hervorgerufene Turbulenz und das Vorhandensein von Hindernissen hauptsächlich turbulenter Natur sind, können die durch Bewegung der Moleküle hervorgerufenen Diffussionsvorgänge gegenüber den turbulenten Diffussionsprozessen vernachlässigt werden. Die gezeigten Berechnungen wurden auf dieser Grundlage durchgeführt.

Die Kompartimentgröße ist nicht das alleinig entscheidende Kriterium. Einen großen Einfluss üben die Randbedingungen aus. Eine unzureichende Abgrenzung z.B. hinsichtlich der Lage von Öffnungen hat eine Fehlverteilung im System und damit eine Fehlaussage zur Folge. Zur Lösung des Gleichungssystems müssen somit die Randbedingungen vorgegeben werden. Stößt ein Kompartiment beispielsweise an eine Wand, so ist der Austauschkoeffizient vom Kompartiment in Richtung Wand gleich Null zu setzen. Dabei werden Spalten, Fugen oder Ritzen in den Begrenzungen vernachlässigt. Öffnungen werden anhand eindeutiger Koordinaten definiert. Die Art bzw. der Zustand der Öffnungen (Spaceboard, Windnetz, angekipptes Fenster, vollständig offene Fläche etc.) wird momentan noch nicht betrachtet. Allein die Berücksichtigung der Fensterneigung ist enorm aufwendig [49].

Die Durchführung der Volumenstrombestimmung bei Annahme einer homogenen Anfangsverteilung der Tracerkonzentration im Stall ist nur dann sinnvoll, wenn definierte Strömungsbedingungen vorliegen, d.h. im Falle einer Zwangslüftung. Der Volumenstrom ist weitgehend unabhängig von den 
äußeren Bedingungen. Der Massenaustrag an gasförmigen Substanzen ist, bei $\dot{V}=$ konstant, proportional zur emittierten Stoffkonzentration.

In Ställen mit freier Lüftung wird der Volumenstrom von einer Vielzahl von Parametern beeinflusst. Den stärksten Einfluss auf das Strömungsgeschehen im Stall übt nach [9] und [13] die Windrichtung aus. Die internen Strömungsverhältnisse werden bei wechselnden Anströmrichtungen erheblich beeinflusst. Eine Übertragbarkeit der Strömungssituation auf andere Windrichtungen ist nicht möglich. Dies bestätigen die gemachten Ausführungen in Kapitel 2.1.4 wonach es sehr schwierig ist, Messpunkte in einem System zu finden, die als Repräsentant den tatsächlichen Strömungsverlauf in diesem System wiedergeben. Die Annahme einer inhomogenen Traceranfangskonzentration ist daher gerechtfertigt. Als Hauptursachen für die unvollständige Vermischung des Tracers mit der Luft werden nach [3] zum einen im System vorhandene stagnierende Bereiche, d.h. Bereiche mit einem geringen Stoffaustausch, und zum anderen sog. "Kurzschlussbereiche" angesehen. Letztere entstehen immer dann, wenn die eintretende Frischluft auf direktem Wege zu den Austrittsöffnungen transportiert wird, ohne einen wesentlichen Beitrag zum Stoffaustausch zu leisten.

Die Positionierung von Messpunkten sollte nach PENG [54] in Gebieten mit vorhandener Rezirkulation erfolgen. Dadurch werden Bereiche geringer Geschwindigkeit (Wände) oder hoher Geschwindigkeit (Eintrittsöffnung der Frischluft) ausgeklammert, in denen mit hoher Wahrscheinlichkeit eine Unter- bzw. Überschätzung des tatsächlichen Luftwechsels erfolgt. Vorteilhafter als die interne Messpunktpositionierung sehen Demmers ET AL. [13] die Positionierung der Messpunkte an den Abluftöffnungen an. Die integrale Aufzeichnung der Tracerkonzentration erwies sich als die genaueste Variante. Betrachtet man aber Stallformen mit langgestreckten seitlichen Öffnungen, wie z.B. sog. Louisianaställe (Abb. 3b)), so ist eine integrale Positionierung der Messpunkte bzw. Berechnung des Luftvolumenstromes sehr schwierig, denn jeder Einlass kann innerhalb kürzester Zeit als Auslass fungieren und umgekehrt. Der Einsatz der Kompartimentalisierungsmethode ist unabhängig von der Position der Messpunkte. Befindet sich ein Messpunkt z.B. in Wandnähe, so ist die Größe des dazugehörigen Kompartimentvolumens entsprechend kleiner. Jedem Messpunkt wird anhand seiner Lage im System ein virtuelles Volumen zugrunde gelegt, innerhalb dessen die Tracerkonzentration als homogen angesehen wird. Durch die Beachtung der Massenerhaltung kann einem Kompartiment in einem Zeitschritt nur eine endliche Masse an Luft zugeführt werden. Bei einer "Fehlpositionierung" würden dadurch höhere Luftwechsel, z.B. in Eintrittsöffnungen, resultieren, die sich aber insgesamt ausgleichen müssen, d.h. durch das Gesamtsystem "geschoben" werden.

Bei geringen Windgeschwindigkeiten $\left(<1 \frac{\mathrm{m}}{\mathrm{s}}\right)$ erhöht sich der Einfluss der Temperatur auf die stallinterne Luftströmung ([2],[35]), wobei auch bei einer Berücksichtigung der Temperatur die Annahme einer homogenen Anfangstemperaturverteilung, analog zur Annahme einer homogenen Traceranfangsverteilung, eine mögliche wärmephysiologische Bewertung des Systems anhand der Temperaturverteilung begrenzt [26]. Höhere Windgeschwindigkeiten ( $>5 \frac{\mathrm{m}}{\mathrm{s}}$ ) verringern nach DEMMERS ET 
AL. [12] die Aussagekraft von Tracermessungen. Als Ursache wird die mit hohen Windgeschwindigkeiten verbundene, unzureichende Vermischung des Tracers angesehen, infolge derer es bei einer Fehlpositionierung mit hoher Wahrscheinlichkeit zu Über- und Unterschätzungen des Luftwechsels kommt. Zudem kann nach [12] eine erzwungene Vermischung des Tracers mit der Stallluft (starkes manuelles Wedeln) eine verstärkte $\mathrm{NH}_{3}$-Freisetzung bewirken. Dem Effekt z.B. einer Überschätzung des Luftvolumenstromes würde ein weiterer hinzugefügt werden, nämlich der einer Überschätzung der Stoffkonzentration an den Konzentrationsmesspunkten.

Nach BARBER UND OGILVIE [4] übt neben den atmosphärischen Einflussfaktoren die Stallgeometrie einen großen Einfluss aus. Dieser kann teilweise von höherer Priorität (Erzeugung etlicher lokaler Raumströmungen) sein als eine unvollständige Vermischung des Tracers. Daher ist es nach [4] erforderlich, mehrere Messpunkte einzusetzen. Je mehr Messpunkte zur Aufzeichnung der Tracerkonzentration zur Verfügung stehen, desto genauer ist die Aussagekraft der Ergebnisse (Kapitel 2.1.4, Abbildungen 7 und 8). Eine höhere Anzahl an Messpunkten ist in der Praxis aber nicht realisierbar.

Erschwerend kommt hinzu, dass bei zu erwartenden Luftwechselzahlen von $\alpha>10 \frac{1}{h}$ das Zeitintervall zwischen zwei Proben unter 2 Minuten liegen sollte [70]. Die Mehrheit der verfügbaren Tracerverfahren bzw. Analysetechniken kann diese Bedingung jedoch nicht erfüllen. Trotz aller Kritik ist die eingesetzte Tracertechnik mit Krypton 85 nach jetzigem Kenntnisstand die einzige Technik, die dieser elementaren Anforderung gerecht wird. Die in Kapitel 5.2 vorgestellte Tracergasmesstechnik stellt momentan die ausgereifteste Technik zur zeitgleichen(!), sekündlichen Aufzeichnung der Tracerkonzentration dar, die im landwirtschaftlichen Bereich eingesetzt wird. Daher ist es nicht zweckmäßig, die Akzeptanz eines (entsprechend dosierten und nicht gesundheitsschädigenden) Tracers in Frage zu stellen, wenn dieser mit der dazugehörigen Messtechnik im Hinblick auf die gesetzte Zielstellung - sichere Quantifizierung des Luftvolumenstromes in landwirtschaftlichen Nutztierställen - den entscheidenden Vorteil aufweist. Durch den möglichen Einsatz von momentan bis zu 80 Sensoren wird eine solide Datenbasis bereitgestellt. Das darin enthaltene Informationspotential wird aber durch die bisher angewendeten Auswertemethoden mit den aufgezeigten Unzulänglichkeiten nicht genutzt. Mit Hilfe der Kompartimentalisierung wird dieses Potential nutzbar gemacht. Es können auch bei begrenzter Zahl an Sensoren, und diese Situation liegt hier vor, Aussagen zum Strömungsgeschehen in einem frei gelüfteten Stall getroffen werden. Das ist die Verbesserung im Vergleich zur Auswertung der Konzentrationsmessreihen mittels Abklingmethode.

Die Anordnung der Sensoren im Untersuchungsraum ist willkürlich. Da die Strömung nicht vorhergesagt werden kann (Kapitel 2.1.4), wird die symmetrische Anordnung als plausibelster Weg beschritten. Die Position der Sensoren wird dabei ebenso wie die Position der Öffnungen und eventueller Stallhindernisse eindeutig in einem kartesischen Koordinatensystem festgelegt. Anhand der Stallabmessungen (Länge, Breite, Höhe bis Trauf, Höhe Trauf bis First) kann nun jedem Sensor ein Volu- 
menanteil zugeordnet werden. Die Größe der dadurch entstehenden Kompartimente ist in der Regel verschieden. Eine vollständige Aufteilung des Untersuchungsraumes in Kompartimente ist aber in der Praxis aufgrund der baulichen Gegebenheiten unmöglich. Es existiert ein nicht zu definierendes Restvolumen $V_{\text {Rest }}$.

Idealfall: $V_{\text {ges }}=\sum_{n} V_{i}$

Realfall: $V_{\text {ges }} \neq \sum_{n} V_{i}$

Die Bestimmung des Stallvolumens bzw. der Summe der einzelnen Kompartimentvolumina erfolgt damit durch:

$$
V_{\text {ges }}=\sum_{n} V_{i}+\underbrace{\varepsilon_{R} \cdot V_{\text {ges }}}_{V_{\text {Rest }}}
$$

wobei $\varepsilon_{R}<1$ und $\mathrm{n}=$ Anzahl der Kompartimente. Der Raum wird in eine bestimmte Anzahl an Kompartimenten aufgeteilt, wohlwissend, dass mit dieser Aufteilung ein Fehler $\varepsilon_{R}$ verbunden ist.

Bei einem Auslass ist der errechnete mittlere Volumenstrom selbstverständlich gleichzeitig dem Volumenstrom aus dieser Austrittsöffnung. Bei mehreren Auslässen, und das ist die Praxis, ist eine Zuordnung von partiellen Volumenströmen zu bestimmten Öffnungen unter Einsatz der Abklingmethode nicht möglich. Dabei ist es unerheblich, ob an jedem Sensor (Ansaugpunkt) ein mittlerer Luftwechselkoeffizient ermittelt wird und dann der gesamte mittlere Volumenstrom errechnet wird oder ob eine mittlere Abklingkonstante (Probenahme) von allen Sensoren ermittelt und dann als mittlerer Luftwechsel interpretiert wird ${ }^{4}$. Der Mittelungsfehler bleibt bestehen. Es sind keine Aussagen zum lokalen Strömungsbild und damit zu Austauschvorgängen an bestimmten Öffnungen möglich. Es wird ein mittlerer Luftwechsel bestimmt, der "irgendwo" das System verlässt.

Die Anwendung der Kompartimentalisierungsmethode zeigt, dass Öffnungen als Eintritts- bzw. Austrittsorte identifiziert werden. Unter Berücksichtigung der Größe der jeweils vorhandenen Austauschflächen kann die mittlere Geschwindigkeit durch diese Fläche berechnet werden. Im Vergleich zur Numerik werden bei der Kompartimentalisierung vor der Rechnung keinerlei Öffnungen als Eintritt oder Austritt festgelegt. Strömungsfeld und -richtung ergeben sich allein durch die Lösung des aufgestellten Gleichungssystems. Der Vorteil besteht in der eindeutigen Zuordnung von Volumenströmen zu bestimmten Öffnungen des Systems. Damit sind nach VAN'T OOSTER [52], der Untersuchungen mittels Druckdifferenzmessungen vornahm, Aussagen zum lokalen Luftwechsel und damit zu definierten Massenströmen möglich.

\footnotetext{
${ }^{4}$ Bei der letztgenannten Vorgehensweise sind überhaupt keine lokalen Aussagen möglich, da die Tracerkonzentrationsbestimmung integrativ über das System durchgeführt wird.
} 
Hinsichtlich der Matrixbestückung muss das zugrundeliegende Gleichungssystem überarbeitet werden. Wie an den Ergebnissen des Stalles in Rothenstadt sichtbar wird, existieren Kompartimente, die über mehr als eine Austauschfläche mit der Umgebung verbunden sind. Hierfür liefert der eingesetzte Householder-Algorithmus keine Lösung. Die gezeigten Ergebnisse entstanden durch das Hinzufügen einer zusätzlichen Randbedingung. Demnach werden bei Kompartimenten mit $>1$ Austauschflächen zur Umwelt alle resultierenden Austauschkoeffizienten zur Umwelt in einem Austauschkoeffizienten zusammengefasst. Der errechnete Luftaustausch des entsprechenden Kompartimentes erfolgt dann ohne Richtungsangabe. Ein weiterer Nachteil kann bei hohen Luftwechseln auftreten, infolgedessen nur kurze Messreihen vorliegen. In diesen Fällen ist es unter Umständen nicht möglich, ausreichend viele Zeitschritte für die Berechnung zur Verfügung zu stellen. Dies ist ersichtlich an der Höhe der errechneten Luftvolumenströme in den Modellversuchen bei einer Bestückung des Bilanzierungsraumes mit 9 bzw. 25 Sensoren. Die Genauigkeit steigt c.p. mit zunehmender Anzahl an Zeitschritten. Andererseits ist es gerade bei der freien Lüftung unwahrscheinlich, dass die Windanströmverhältnisse vor Ort konstant bleiben. Eine Erhöhung der Anzahl der Bestimmungsgleichungen muss bei stark wechselnden Windverhältnissen sorgfältig abgewogen werden.

Rechenmodelle müssen die grundlegenden physikalischen Prinzipien, z.B. die Massenerhaltung, erfüllen. Die formale Modellprüfung wird auch als Verifikation bezeichnet. Die Berechnung des Volumenstromes erfolgt unter Berücksichtigung der behandelten strömungsmechanischen Gesetzmäßigkeiten in Kapitel 3. Für jedes Kompartiment wird eine Bilanzgleichung erstellt. Diese beschreibt die Änderung der Tracerkonzentration im Kompartiment im Verhältnis zum Tracergasaustausch mit den benachbarten Kompartimenten. Es wird ferner angenommen, dass ein Massenaustausch an Tracer zu einem Zeitpunkt nur über eine (gedachte) Kompartimentsgrenze erfolgen kann. Dieses wird durch die hohe zeitliche Aufeinanderfolge der Messwerteerfassung sichergestellt.

Unter Validierung eines Modells versteht man den Vergleich der Modellergebnisse mit messtechnisch ermittelten Werten. Diese Werte werden beispielweise bei der Geruchsimmissionsprognose direkt in die Modelle eingebracht, d.h. die Validierung erfolgt direkt durch die Anpassung des Modells an die gemessenen Werte. Im Rahmen der Arbeit stellen die Werte die gemessenen Konzentrationsreihen des Tracers dar. Ausgehend von den "unbehandelten" Messdaten wird der Volumenstrom des Systems, d.h. an den Zu- und Abluftöffnungen, sowie zwischen den Kompartimenten bestimmt. Die integrale Betrachtungsweise (bezogen auf das Kompartiment) erlaubt es, die Ortsabhängigkeit des Skalars Tracerkonzentration zu vernachlässigen, d.h. der Einfluss instabiler Anströmgeschwindigkeiten und -richtungen muss nicht explizit berücksichtigt werden [18]. Somit bleibt auch bei der freien Lüftung nur die zeitliche Abhängigkeit der Tracerkonzentration bestehen. Aus diesem Grund ist ein Vergleich mit herkömmlichen Tracerverfahren nur bedingt möglich, da die Ausgangsbasis in Form der zusätzlichen örtlichen Abhängigkeit in diesen Verfahren eine andere ist. 
VAN' T OOSTER [52] ermittelte eine durchschnittliche Ammoniakemissionsrate über einen Zeitraum von 190 Tagen von 1, $5 \frac{g}{G V \cdot h}$. Es handelte sich um einen Boxenlaufstall mit Traufen-First-Lüftung. Die Entmistung erfolgte über Spaltenböden mit darunterliegendem Güllekeller. Ebenfalls über 190 Tage wurden $\mathrm{NH}_{3}$-Emissionen von DEMMERS ET AL. [12] in einem Boxenlaufstall mit senkrechten Lüftungsschlitzen (Spaceboard) bestimmt. Sie betrugen im Durchschnitt $1,3 \frac{g}{G V \cdot h}$. In ähnlicher Größenordnung liegen die Ammoniakwerte von SEEDORF ET AL. [65]: 1, $0 \frac{g}{G V \cdot h}$. Den Untersuchungen lag ebenfalls ein Boxenlaufstall mit Traufen-First-Lüftung und Spaltenböden zugrunde.

SEIPELT [66] untersuchte in zwei Wintermessperioden insgesamt vier Milchviehställe mit TraufenFirst-Lüftung, die jeweils unterschiedliche Entmistungsformen aufwiesen. Die Höhe der $\mathrm{NH}_{3}$-Emissionen reichte von $1,6 \frac{g}{G V \cdot h}$ aus einem Boxenlaufstall mit Topboden bis hin zu $3,6 \frac{g}{G V \cdot h}$ aus einem Tretmiststall. Hinsichtlich der Berechnung der Methanemissionen lagen die errechneten Werte zwischen $11,1 \frac{g}{G V \cdot h}$ aus einem Boxenlaufstall mit befestigter Trittfläche und Klappschieber und 32, $6 \frac{g}{G V \cdot h}$ wiederum bei der Tretmistvariante. Mit Ausnahme der Ergebnisse von SEEdorf ET AL. [65] - Luftvolumenstromberechnung mittels $\mathrm{CO}_{2}$-Bilanz - wurde in allen anderen aufgeführten Literaturbeispielen die Abklingmethode zur Ermittelung des Luftvolumenstromes verwendet.

Die Ergebnisse der eigenen Arbeit weisen ähnliche Massenströme an Ammoniak und Methan auf. Dabei muss betont werden, dass es sich nicht um Durchschnittsangaben sondern um Momentangaben handelt. Zusätzlich zu den bisherigen Ergebnissen aus der Literatur ist es mit Hilfe der Kompartimentalisierungsmethode erstmals möglich, das Strömungsgeschehen im Stall in Form der errechneten Austauschkoeffizienten sichtbar werden zu lassen. Neben der rein integrativen Angabe des Emissionsmassenstromes können verfeinerte, differentielle Aussagen zum Luftaustausch innerhalb des Stalles getroffen werden. Somit wird eine Art "Individualbezug" für jeden einzelnen Zeitschritt erzielt.

Mit Hilfe der CFD-Technik konnte eine "Kalibrierung" der Kompartimentalisierungsmethode und der Abklingmethode durchgeführt werden. Die betrachteten Modellbeispiele spiegeln nicht die Realität wieder. Die vielfach komplexeren Strömungsvorgänge in Praxisställen können derzeit nicht vollständig berücksichtigt werden; der hierzu erforderliche Zeitaufwand ist immens. CFD-Simulationen sind nach [13] zur Vorhersage der Positionierung von Messpunkten nur eingeschränkt einsetzbar, da sie selbst einer Verifizierung bedürfen. Dennoch erweisen sich CFD-Simulationen im Hinblick auf das hier gesetzte Ziel - Bestimmung des Luftvolumenstromes in relativ einfachen Fällen unter kontrollierten Bedingungen - als geeignetes Hilfsinstrument.

Die Kompartimentalisierungsmethode erhebt nicht den Anspruch eines Strömungssimulationsprogramms. Im Vergleich zu numerischen Simulationsrechnungen sind die Kontrollvolumina bei der Kompartimentalisierung ungleich größer, das Gitter weitaus grobmaschiger. Detaillierte Aussagen "bis in die kleinste Stallecke" können und sollen nicht getroffen werden. Eine Referenzmethode, die eine Validierung der Tracertechnik bei Feldmessungen ermöglicht, gibt es nicht. Es wird anhand 
der aufgezeigten, klaren physikalischen Vorgehensweise aber deutlich, wo die Unzulänglichkeiten herkömmlicher Verfahren zu finden sind und in welchem Umfang die damit gewonnenen Ergebnisse interpretiert werden dürfen.

Simulationen von AXLEY [1], die in einem zweidimensionalen Raum von 3 m x 9 m durchgeführt wurden, bestätigen die eigene Aussage, dass die Kompartimentalisierungsmethode prinzipiell nicht geeignet ist, das tatsächliche turbulente Strömungsgeschehen wiederzugeben. Dennoch ist sie in der Lage, die wesentlichen Strömungsverläufe zu deuten [1]. Das unterstreicht die Zielsetzung dieser Arbeit. Sie bestand darin, die beschränkte Tracermesstechnik so einzusetzen, dass realistische Aussagen zum Luftwechsel möglich sind. Es ist der Versuch, die bislang gängigen Verfahren qualitativ zu verbessern. Dabei erfolgte bewusst die Anlehnung an die Strömungsmechanik, um nicht den zweiten Schritt (der Ergebnispräsentation) vor dem ersten Schritt (der Vorgehensweise) zu tun.

Um die vorliegende Arbeit im Hinblick auf die landwirtschaftliche Nutzanwendung beurteilen zu können, sind einige weiterführende Betrachtungen erforderlich. Bei Genehmigungsverfahren in der Landwirtschaft ist die auf der Ermächtigungsgrundlage des Bundes-Immissionsschutzgesetzes (BImSchG, § 48) beruhende Verwaltungsvorschrift “Technische Anleitung zur Reinhaltung der Luft” (TALuft) bindend. Darin wird u.a. auf die entsprechenden VDI-Richtlinien verwiesen. Aufgrund des unzureichenden Beurteilungsschemas der außer Kraft gesetzten VDI-Richtlinie 3473 in Bezug auf frei gelüftete Rinderställe besteht großer Handlungsbedarf. Gerade im Bereich der Rinderhaltung dominieren die natürlich gelüfteten Stallanlagen, die sich zudem oft in unmittelbarer Nähe von Wohngebieten befinden. Es mangelt an verlässlichem Datenmaterial über die tatsächliche Höhe der Emissionsmassenströme aus den genannten Anlagen.

$\mathrm{Zu}$ beurteilende Geruchsimmissionen sind infolge der Transmission von Geruchsstoffen an die Emission geknüpft. Emittiert wird dabei aus dem System Stall der mit der Quellkonzentration $C_{\text {geruch }}$ beladene Volumenstrom $\dot{V}$. Im weiteren Verlauf wird dieser aus dem Stall emittierte Geruchsstoffmassenstrom durch den atmosphärischen Wind in der Umgebung verbreitet und verdünnt. Die Ausbreitung von Gerüchen in der atmosphärischen Grenzschicht wird durch das sogenannte Gauß-Modell ${ }^{5}$ beschrieben. Hiernach hängt die Geruchsimmission direkt vom emittierten Massenstrom aus dem Stall ab, d.h. die Geruchsimmissionskonzentration im direkten Umfeld einer Quelle ist proportional zu Geruchsemissionskonzentration. Fehler in der emissionsseitigen Massenstrombestimmung wirken sich direkt auf den immissionsseitigen Massenstrom aus. Damit ist die Brücke zur eingangs gestellten Fragestellung geschlagen: Wenn ein mittlerer Massenstrom eines luftfremden Stoffes bestimmt werden soll, ist dann der Fehler, der durch die nicht zeitgleiche Mittelung des Produktes $C(t) \cdot \dot{V}(t)$ produziert wird, größer, als wenn die Faktoren getrennt gemittelt werden und dabei der Volumenstrom

\footnotetext{
5 Auf die fehlende Berücksichtigung der Umgebungsbebauung eines Stalls, die diesem Modell anhaftet, wird hier nicht eingegangen.
} 
durch die pauschale Mittelung im Strömungsraum berechnet wird?

In Anlehnung an Gleichung 6 kann die zeitliche Änderung des Gesamtmassenstromes bezogen auf die zeitliche Änderung der Konzentration und des Luftvolumenstromes wie folgt beschrieben werden:

$$
\begin{aligned}
\delta \dot{m} & \approx \frac{\partial \dot{m}}{\partial C} \cdot \delta C+\frac{\partial \dot{m}}{\partial \dot{V}} \cdot \delta \dot{V} \\
& =\dot{V} \cdot \delta C+C \cdot \delta \dot{V}
\end{aligned}
$$

bezogen auf den Massenstrom $\dot{m}$ folgt aus Gleichung 84

$$
\begin{aligned}
\underbrace{\frac{\delta \dot{m}}{\dot{m}}} & =\frac{\dot{V}}{\dot{m}} \cdot \delta C+\frac{C}{\dot{m}} \cdot \delta \dot{V} \\
\varepsilon_{\dot{m}} & =\underbrace{\frac{\delta C}{C}}+\underbrace{\frac{\delta \dot{V}}{\dot{V}}} \\
\varepsilon_{\dot{m}} & \approx \varepsilon_{C}+\varepsilon_{\dot{V}}
\end{aligned}
$$

Der Messfehler in der Massenstrombestimmung setzt sich nach Gleichung 87 aus der Summe der Fehler in der Konzentrations- und Luftvolumenstrombestimmung zusammen. Dazu ein Beispiel:

In einem Rinderstall beträgt die Geruchsstoffkonzentration $C=500 \frac{G E}{m^{3}}$. GE steht für Geruchseinheit. Der dazugehörige Luftvolumenstrom beträgt $\dot{V}=150.000 \frac{\mathrm{m}^{3}}{\mathrm{~h}}$. In der Praxis sind Abweichungen in der Bestimmung der Geruchsstoffkonzentration von $\pm 100 \frac{G E}{\mathrm{~m}^{3}}$ und im Luftvolumenstrom von $\pm 20.000 \frac{m^{3}}{h}$ durchaus keine Seltenheit. Der Messfehler in der Massenstrombestimmung ergibt sich anhand der gemachten Annahmen

$C=500 \frac{G E}{m^{3}} ; \delta C= \pm 100 \frac{G E}{m^{3}} ;\left|\frac{\delta C}{C}\right|=0,2$

$\dot{V}=150.000 \frac{m^{3}}{h} ; \delta \dot{V}= \pm 20.000 \frac{m^{3}}{h} ;\left|\frac{\delta \dot{V}}{\dot{V}}\right|=0,13$

zu Gleichung 88

$$
\begin{aligned}
\delta \dot{m} & =150.000 \cdot 500 \cdot 0,2+150.000 \cdot 500 \cdot 0,13 \\
& =0,33 \cdot \dot{m}
\end{aligned}
$$

Im angenommenen Fall kann somit ein Messfehler von $33 \%$ auftreten. Mit Blick auf Gleichung 8 ist es aber unerheblich, welcher der beiden Faktoren in der Massenstrombestimmung mit einem Fehler 
behaftet ist. Das heißt, es kann nicht rückvollzogen werden, ob der Fehler in der Konzentrationsbestimmung oder in der Luftvolumenstrombestimmung begangen wurde. Dafür ist der Fehlerbereich mit $0<\left|\varepsilon_{C}\right|$ bzw. $0<\left|\varepsilon_{\dot{V}}\right|$ zu groß.

Der Nutzen der Arbeit besteht in der erstmaligen verfeinerten Abschätzung von $\varepsilon_{\dot{V}} \cdot \varepsilon_{C}$ rekrutiert sich dabei aus der olfaktometrischen Messmethode. Er ist auf direktem Wege nicht abschätzbar, nur durch einen Probandenvergleich. Da zu dieser Auswertemethode keine Alternative besteht, wird die ermittelte Geruchskonzentration übernommen. Damit haftet $\varepsilon_{C}$ allen Verfahren zur Massenstromberechnung an. Hinsichtlich des Fehlers $\varepsilon_{\dot{V}}$ gelang es, eine Spannbreite zu ermitteln. Unter Berücksichtigung der theoretischen Modellversuche und der Praxisversuche liegt diese Fehlerspannbreite bei $30 \%$ bzw. 22, $5 \%$. Das bedeutet, der Fehlerbereich für die Luftvolumenstrombestimmung mittels Abklingmethode kann mit $0<\left|\varepsilon_{\dot{V}}\right|<0,3$ neu definiert werden. 


\section{Zusammenfassung}

Der landwirtschaftliche Bereich hat im Hinblick auf strömungsmechanische Berechnungen keine Sonderstellung inne. Die prinzipiellen physikalischen Gesetzmäßigkeiten gelten auch für die Erfassung von Luftmassenströmen in natürlich gelüfteten Stallgebäuden. Diese Stallgebäude können als Hindernisse in der freien atmosphärischen Strömung betrachtet werden. Durch das Vorhandensein von Austauschflächen zur Umgebung erfährt der "Hohlkörper Stall” zusätzlich zur Umströmung eine Durchströmung. Die je nach den Windanströmverhältnissen durch Druckdifferenzen erzeugten Luftströmungen bewirken den Massenaustrag an stalltypischen gasförmigen Substanzen von innen nach außen. Ziel der vorliegenden Arbeit ist es, diesen Massenaustrag zu quantifizieren.

Dazu wurde, neben der Konzentrationsbestimmung, das Kompartimentalisierungsverfahren zur Luftvolumenstrombestimmung eingesetzt, welches im Ansatz im medizinischen Bereich bereits seit Jahrzehnten angewendet wird. Es kann in diesem Zusammenhang als eine Art Zwischenlösung zwischen numerischer Strömungssimulation und integraler Volumenstrombestimmung angesehen werden. Bei diesem Verfahren werden vereinfachender Weise mathematisch komplexe Vorgänge in linearisierten Gleichungssystemen zusammengefasst und gelöst.

Basis der hier angewendeten Kompartimentalisierungsmethode, welche in eine eigene Software eingebettet ist, sind in der Praxis gewonnene Messwerte. Dies unterstreicht die Notwendigkeit der praktischen Messtechnik. In der Auswertung der gewonnenen Messdaten weist die Kompartimentalisierungsmethode grundsätzliche Unterschiede im Vergleich zu üblichen Tracermessmethoden auf. Insbesondere berücksichtigt die Kompartimentalisierungsmethode:

- die Erhaltungsgleichungen; es wird nur auf die Massenerhaltung Bezug genommen

- die Annahme einer inhomogenen Konzentrationsanfangsverteilung des Tracergases im Untersuchungsraum

- die zeitliche Konzentrationsänderung in den Kompartimenten bei der schrittweisen Optimierung der Austauschkoeffizienten

Das Kompartimentalisierungsverfahren dient der Ergänzung der Ergebnisse von Tracergasmessungen. Dadurch ist z.B. die Berechnung des Strömungsfeldes für den gesamten Stall möglich. Damit lassen sich Volumenstromberechnungen durchführen, mit denen wiederum Angaben über die Massenströme der betrachteten Gase möglich sind. Diese sind, bezogen auf Geruchsstoffe, direkt an die Immissionsseite geknüpft. Die Massenströme liefern objektive Kriterien bei der Fragestellung, ob eine Tierhaltungsanlage genehmigungsfähig ist oder nicht. 
Aus der Vielzahl von Stallsystemen der natürlichen Lüftung wurden stellvertretend zwei Lüftungssysteme untersucht. Die Tracerkonzentration (Tracer: Kr 85) wurde über einen Zeitraum von einigen Minuten parallel an mehreren Messpunkten aufgezeichnet. Diese Zeitreihen der Tracerkonzentration dienten der Berechnung der Luftvolumenströme und ferner der Emissionsmassenströme an Ammoniak, Methan, Kohlendioxid, Lachgas und Wasser aus beiden untersuchten Stallsystemen.

Die Ergebnisse der vorliegenden Arbeit zeigen, dass der Milchviehstall mit Schwerkraftlüftung in Faulenhorst (Offenstall mit Monoschacht) $3 g \mathrm{NH}_{3}, 31 g \mathrm{CH}_{4}, 1.600 g \mathrm{CO}_{2}, 0,2 g \mathrm{~N}_{2} \mathrm{O}$ und $8.700 g$ $\mathrm{H}_{2} \mathrm{O}$ pro GV und Stunde freisetzt. Die Hochrechnung der Massenströme auf den Zeitraum von einer Stunde setzt selbstverständlich die Annahme konstanter Randbedingungen (Windgeschwindigkeit, Windrichtung) für diesen Zeitraum voraus. Diese Berechnungen stellen keinen Durchschnittswert dar sondern Momentaufnahmen, die die tatsächlichen Strömungsverhältnisse wiedergeben. Insgesamt wurden Emissionsmassenströme für einen Untersuchungszeitraum von 32 Sekunden berechnet. Das diskutierte Strömungsbild gilt für einen willkürlich gewählten Zeitschritt.

Der Milchviehstall in Rothenstadt, welcher als Offenfrontstall ausgelegt ist, emittiert $0,9 \mathrm{~g} \mathrm{NH} 3,24 \mathrm{~g}$ $\mathrm{CH}_{4}, 5.750 g \mathrm{CO}_{2}, 4 g \mathrm{~N}_{2} \mathrm{O}$ und $35.000 \mathrm{~g} \mathrm{H} \mathrm{H}_{2} \mathrm{O}$ pro $\mathrm{GV}$ und Stunde. Für die Hochrechnung der Massenströme auf den Zeitraum von einer Stunde gelten die o.g. Ausführungen. Diese Berechnungen stellen ebenfalls keinen Durchschnittswert sondern Momentaufnahmen dar. In diesem Fall werden Emissionsmassenströme für einen Untersuchungszeitraum von 31 Sekunden berechnet. Das diskutierte Strömungsbild gilt ebenfalls für einen willkürlich gewählten Zeitschritt. Im Vergleich zu dem Stall mit Öffnungsschacht liegen die Ammoniakkonzentrationen in dem Offenfrontstall an der unteren Nachweisgrenze. Die Lachgas-, Kohlendioxid- und Wasserdampfemissionsraten sind in diesem Stallsystem aber deutlich höher.

Ein Offenfrontstall stellt hinsichtlich der Strömungsverhältnisse die komplizierteste Bauform aller frei gelüfteten Ställe dar. Deshalb sind anspruchsvolle Berechnungsgeometrien bei einer Volumenstrombestimmung erforderlich. Dies zeigen die Ergebnisse in Kapitel 6. Luftwechselangaben, die mittels Abklingmethode ermittelt werden, müssen in diesen Fällen besonders sorgfältig geprüft werden.

Anhand der parallel durchgeführten, theoretischen Modellversuche kann ein Vergleich von Abklingund Kompartimenteverfahren vorgenommen werden. Dazu wurde die numerische Strömungssimulation heran gezogen, deren Lösung als exakt anzusehen ist. Die errechneten Luftwechselzahlen nach der Abklingmethode liegen stets unterhalb der tatsächlichen Lüftungsraten. Die Ergebnisse der Kompartimentalisierungsmethode stimmen dagegen gut mit den tatsächlichen Werten überein.

Die numerische Simulationsrechnung ist überaus rechenintensiv, so dass eine Beschränkung auf vereinfachte Fälle erforderlich ist, die jedoch den vielfach komplexeren Verhältnissen in der Praxis nicht gerecht wird. Um die gewonnenen Ergebnisse zu validieren, werden deshalb Messungen in Realställen durchgeführt. Praxismessungen stellen ohnehin die einzige glaubwürdige Möglichkeit dar, um theoretische Ergebnisse zu überprüfen. 
Die Messungen bestätigen die erzielten Ergebnisse. Auch hier liegen die Volumenstromwerte nach der Abklingmethode unter denen der Kompartimentalisierungsmethode. Eine Messauswertung mittels Abklingmethode führt damit zu einer Unterschätzung des tatsächlichen Volumenstromes. Mit Hilfe der Kompartimentalisierung können die Ergebnisse des Abklingverfahrens überprüft und gegebenenfalls korrigiert werden. Im Hinblick auf konventionelle Tracerverfahren ist bei nicht zwangsventilierten Ställen zudem eine Erfassung der meteorologischen Parameter unabdingbar. Nur hierdurch kann eine realitätsnahe Fehlerabschätzung vorgenommen werden.

Die vorliegende Untersuchung belegt, dass sich auch bei einer begrenzten Anzahl von Sensoren, großen Stallvolumina und unzureichender Vermischung des Tracers quantitative Aussagen über Volumenströme bzw. Massenströme aus natürlich gelüfteten Tierhaltungsanlagen treffen lassen. 


\section{Summary}

There is no evidence that the physical laws of fluid mechanics don't hold true for agricultural area. They are applied in the following to calculate air flow rates in stables with natural ventilation. Such stables can be regarded as obstacles in the air flow of free atmosphere. This obstacle is a 'hollow body' with openings, which allow air flow through and around. The pressure differences and further the air flow pattern depending by the air stream conditions cause the exchange of airborne stable emissions like ammonia or odour into the neighbourhood. The objective of this thesis is to quantify the mass flow of these substances.

For this - besides the measurement of the gas concentrations - the method of compartmentalisation was applied which is implemented in an own software. The compartmentalisation has been used specially in the medical area since some decades. It can be considered as an intermediate solution between the numerical simulation and an integrative air flow calculation. To do so complex mathematical procedures are simplified by a system of linear equations, which are solved by a suitable algorithm.

The compartmentalisation is based on measured data. Therefore practical measurement techniques are indispensable. But there are fundamental differences in evaluation of the measured data between the method of compartmentalisation and tracer technique. In comparison to the tracer decay method, the compartmentalisation method considers:

- The conservation equations. From the three extensive properties mass, momentum and energy, the mass balance is considered.

- An inhomogeneous initial distribution of the tracer concentration in the whole stable.

- A time dependent change of tracer concentration in the compartments for the iterative optimisation of the transfer coefficients.

The compartmentalisation method is used to supplement the results of the tracer measurements. This for example allows the calculation of air flow patterns for a whole stable. It also enables to calculate the air flow and from this the mass flow of the considered gases. Mass flow calculations are of particular importance in case of odour emissions. They provide the basis for objective criteria for approval procedures.

The method of compartmentalisation permits essentially instantaneous values of air flow rate to be determined. Tracer concentration data were obtained from two different stables over a period of a few minutes. These data were used to calculate the air flows in the two stables and the emission rates 
of ammonia, methane, carbon dioxid, nitrious oxide and water over a period of approximately 30 seconds.

The emission rates for the first stable (open dairy stable with monoslot in Faulenhorst) were $3 g \mathrm{NH}_{3}$, $31 \mathrm{~g} \mathrm{CH}_{4}, 1.600 \mathrm{~g} \mathrm{CO}_{2}, 0,2 \mathrm{~g} \mathrm{~N}_{2} \mathrm{O}$ and $8.700 \mathrm{~g} \mathrm{H}_{2} \mathrm{O}$ per livestock unit ( $\mathrm{GV}=500 \mathrm{~kg}$ live weight) and hour. The emission rates for the second stable (open-front dairy stable in Rothenstadt) were $0,9 \mathrm{~g}$ $\mathrm{NH}_{3}, 24 g \mathrm{CH}_{4}, 5.750 \mathrm{~g} \mathrm{CO} \mathrm{CO}_{2}, 4 \mathrm{~N}_{2} \mathrm{O}$ and $35.000 \mathrm{~g} \mathrm{H} \mathrm{H}_{2} \mathrm{O}$ per livestock unit $(\mathrm{GV})$ and hour. It is emphasized that the data are valid only for the particular stables considered and for the particular wind conditions that were present at the time of measurement. The discussed air flow patterns are chosen for an arbitrary time step.

The ammonia concentration in the open-front stable was found to be much lower (close to the natural background concentration) than in the open stable. But the concentrations of nitrous oxide, carbon dioxide and water were clearly higher.

With regard to wind velocity and wind direction an open-front stable is the most crucial geometry for calculation of the air flow; which is shown in Chapter 6. In this case results which are obtained by the tracer decay method have to be interpreted carefully.

The solution of the numerical simulation can be considered to be exact and serves as reference for the compartmentalisation method and the tracer decay method. The simulation shows, that the results of the tracer decay method are permanently too low. The results of compartmentalisation show a good agreement with the simulation.

The numerical simulation demands high computer power. The available computer power is not sufficient to calculate the complex situation of a real stable. Therefore, to validate the results, measurements - the only link to reality - were made in real stables. Also the measured data show the trend, that the air flow calculated using the tracer decay method is too low. With the help of compartmentalisation the results of the tracer method can be validated and corrected, if necessary.

The results of the thesis show that the compartmentalisation method also gives reasonable results concerning the air volume and mass flow in stables with natural ventilation, even if the number of sensors to measure the tracer concentration is limited, the size of the stable under consideration is big and the tracer distribution is not homogeneous. 


\section{Literatur}

[1] AXLEY, J.W. (2000): Zonal models using loop equations and surface drag cell-to-cell flow relations. In: Roomvent 2000, Ventilation for health and sustainable environment, Proceedings of the 7th international conference on air distribution in rooms (Vol I), 9 - 12 July, Reading, United Kingdom: 235-240

[2] Baptista, F.J.; Bailey, B.J.; Randall, J.M.; Meneses, J.F. (1999): Greenhouse ventilation rate: theory and measurement with tracer gas techniques. J. agric. Engng. Res. 72: 363-374

[3] Barber, E.M. And Ogilvie, J.R. (1984): Incomplete mixing in ventilated air spaces. Part II scale-model study. Can. Agric. Engng. 26: 189-196

[4] BARber, E.M. And OGILvie, J.R. (1984): Interpretation of tracer gas experiments in ventilation research. J. agric. Engng. Res. 30: 57-63

[5] BAthe, K.-J. (1990): Finite-Elemente-Methoden: Matrizen und lineare Algebra, die Methode der finiten Elemente, Lösung von Gleichgewichtsbedingungen und Bewegungsgleichungen, Springer Verlag, Berlin

[6] Brehme, G.; Krehl, H.; Müller, H.-J.; Krause, K.-H. (1998): Untersuchung strömungstechnischer Vorgänge im Windkanal mittels Laserlichtschnittmethode am Rhenalux-First 150 GL. Institut für Technologie und Biosystemtechnik, FAL Braunschweig, Institutsbericht, (unveröffentlicht)

[7] BRown, R.F. (1980): Compartmental system analysis: state of the art. IEEE Transactions on Biomedical Engineering 27(1): 1-11

[8] Buffington, R. und Wilson, M.K. (1989): Detektoren für die Gaschromotograhie. Deutsche Ausgabe (Vömel, R. und Wendt, J.), Hewlett-Packard GmbH, Bad Homburg

[9] Caspary, V.; Hinz, T.; Krause, K.-H.; Linke, S. (2000): Numerical modelling of aerial pollutant emissions in and from livestock buildings. Landbauforschung Völkenrode 1(2): 76-84

[10] Cobelli, C. And Romanin-Jacur, G. (1976): Controllability, observability and structural identifiability of multi input and multi output biological compartmental systems. IEEE Transactions on Biomedical Engineering 23(2): 93-100

[11] Choinière, Y. And Munroe, J.A. (1994): A wind tunnel study of wind direction effects on airflow patterns in naturally ventilated swine buildings. Can. Agric. Engng. 36: 93-101 
[12] Demmers, T.G.M.; Burgess, L.R.; Short, J.L.; Phillips, V.R.; Clark, J.A.; WATHES, C.M. (1998): First experiences with methods to measure ammonia emissions from naturally ventilated cattle buildings in the U.K.. Atmospheric Environment 32(3): 285-293

[13] Demmers, T.G.M.; Burgess, L.R.; Phillips, V.R.; Clark, J.A.; Wathes, C.M. (2000): Assessmet of techniques for measuring the ventilation rate, using an experimental building section. J. agric. Engng. Res. 76: 71-81

[14] Deuflhard, P. Und Hohmann, A. (1993): Numerische Mathematik I. Eine algorithmisch orientierte Einführung, de Gruyter Verlag, Berlin

[15] DietzE, L. (1987): Freie Lüftung von Industriegebäuden. VEB Verlag für Bauwesen, Berlin

[16] Eberhardt, L.L.; Gilbert, R.O.; Hollister, H.L.; Thomas, J.M. (1976): Sampling for contaminants in ecological systems. Environmental Science \& Technology 10(9): 917-925

[17] ECKHARDT, H. (1978): Numerische Verfahren in der Energietechnik. Teubner Verlag, Wiesbaden

[18] Enai, M.; Aratani, N; Shaw, C.Y.; Reardon, J.T. (1992): Differential and integral method for computing interzonal airflows using multiple tracer gases. In: International symposium on room air convection and ventilation effectiveness (ISRACVE), University of Tokyo, 22.-24. July: $540-545$

[19] Engeln-Müllges, G. Und Reutter, F. (1991): Formelsammlung zur numerischen Mathematik mit Quick-BASIC-Programmen. BI-Wissenschaftsverlag, Mannheim

[20] FAth, B.D. AND PATten, B.C. (1998): Network synergism: emergence of positive relations in ecological systems. Ecological Modelling 107: 127-143

[21] Ferziger, J.H.; PERIĆ, M. (1997): Computational methods for fluid dynamics. Springer Verlag, Berlin

[22] FIEDLER, O. (1992): Strömungs- und Durchflussmesstechnik. Oldenbourg Verlag, München

[23] Froehlich, D.P.; Hellickson, M.A.; Young, H.G. (1975): Ridge vent effects on model ventilation characteristics. Transactions of the ASAE 18: 690-693

[24] Gardin, P. and Fontaine, J.R. (1990): General ventilation characterisation. In: Roomvent '90, Engineering aero- and thermodynamics of ventilated room, Second International Conference, Oslo, Norway 13.-15. June, Session C-3

[25] Gersten, K. (1991): Einführung in die Strömungsmechanik. Vieweg Verlag, Braunschweig 
[26] GLÜCK, B. (1997): Neuartiges inneres Raummodell. Ki Luft- und Kältetechnik 33(1): 11-16

[27] Grot, A.G. And Lagus, P.L. (1991): Application of tracer gas analysis to industrial hygiene investigations. In: Air movement and ventilation control within buildings, Proc. of the 12th AIVC Conference, Ottawa, Canada 24.-27. September, Vol. 1, Paper 27: 411-471

[28] HÄFner, F.; SAmes, D.; Voigt, H.-D. (1992): Wärme- und Stofftransport. Mathematische Methoden. Springer Verlag, Berlin

[29] Hanel, B. (1994): Raumluftströmung. C. F. Müller Verlag, Heidelberg

[30] Hesse, M.; Meier, H.; ZeEh, B. (1984): Kombination von IR-Spektrometern mit anderen Geräten. In: Spektroskopische Methoden in der organischen Chemie: 84-86, Georg-Thieme Verlag, Stuttgart

[31] JANSSEN, J. (1989): Berechnung turbulenter Luftströmungen in Fahrerkabinen. Dissertation, Fachbereich Energie-, Verfahrens- und Elektrotechnik, Universität Essen

[32] Janssen, J. Und KRause, K.-H. (1987): Stallinterne Beeinflussung der Gesamtemission aus Tierhaltungen. Grundlagen der Landtechnik 37(6): 213-220

[33] JAQUEZ, J.A. (1972): Compartmental analysis in biology and medicine: kinetics of distribution of tracer-labeled materials. Elsevier Publishing Company, Amsterdam

[34] Kittas, C.; Boulard, T.; Mermier, M.; Papadakis, G. (1996): Wind induced air exchange rates in a greenhouse tunnel with continuous side openings. J. agric. Engng. Res. 65: 37-49

[35] Kittas, C.; Boulard, T.; PApadakis, G. (1997): Natural ventilation of a greenhouse with ridge and side openings: sensitivity to temperature and wind effects. Transactions of the ASAE 40(2): 415-425

[36] Krause, K.-H. Und JAnssen, J. (1989): Kontinuierliche Ammoniakmessungen in Ställen. Grundlagen der Landtechnik 39(2): 52-65

[37] Krause, K.-H.; Hake, H.; MaCK, H.-D.; Pardylla, F. (1991): Emissionen und Immissionen von Offenställen (sog. Louisiana-Ställen) im Zusammenhang mit dem BundesImmissionsschutzgesetz. Bundesforschungsanstalt für Landwirtschaft (FAL), BraunschweigVölkenrode, Institut für Biosystemtechnik, Institutsbericht KR03/91

[38] Krause, K.-H. (1999): Persönliche Mitteilung.

[39] Lege, T.; Kolditz, O.; Zielke, W. (1996): Strömungs-und Transportmodellierung. In: Handbuch zur Erkundung des Untergrundes von Deponien und Altlasten (Band 2), BGRBundesanstalt für Geowissenschaften und Rohstoffe, Springer Verlag, Berlin 
[40] Leonard, J.J.; Feddes, J.J.R.; MCQuitTy, J.B. (1984): Measurement of ventilation rates using a tracer gas. Can. Agric. Engng. 26(1): 49-51

[41] Lilek, Ž.; MuZaferija, S; Perić, M.; SeidL, V. (1997): An implicit finite-volume method using non-matching blocks of structured grid. In: Numerical heat transfer (Part B) 32(4): 385-401

[42] LindnER, G. (1990): IR-Spektroskopie. In: Untersuchungsmethoden in der Chemie, Einführung in die moderne Analytik, Kapitel 11: 163-175, Georg-Thieme Verlag, Stuttgart

[43] Logofet, D.O. (1997): Svicobians of the compartment models and DaD-stability of the Svicobians: aggregating '0-dimensional' models of global biogeochemical cycles. Ecological Modelling 104: 39-49

[44] MARSAL, D. (1989): Finite Differenzen und Elemente. Numerische Lösung von Variationsproblemen und partiellen Differentialgleichungen, Springer Verlag, Berlin

[45] Miguel, A.F.; VAn de BraAk, N.J.; Silva, A.M.; Bot, G.P.A. (1998): Physical modelling of natural ventilation through screens and windows in greenhouses. J. agric. Engng. Res. 70: 165176

[46] Mistriotis, A; De Jong, T.; Wagemans, M.J.M.; Bot, G.P.A. (1997): Computational Fluid Dynamics (CFD) as a tool for the analysis of ventilation and indoor microclimate in agricultural buildings. Netherlands Journal of Agricultural Science 45: 81-96

[47] MÜller, H.-J.; GläSER, M.; KuHN, E. (1995): Luftwechselbestimmung mit Tracergasen. Landtechnik 50(3): 166-167

[48] Müller, H.-J.; Möller, B. (1998): Weiterentwickelte Luftwechselmesstechnik mit TracerAnwendung in Tierhaltungen. Landtechnik 53(5): 326-327

[49] NÄÄs, I.A.; MourA, D.J.; BuCKlin, R.A.; Fialho, F.B. (1998): An algorithm for determining opening-effectiveness in natural ventilation by wind. Transactions of the ASAE 41(3): 767-771

[50] Nederhoff, E.M.; VAn de Vooren, J.; Udink ten CATe, A.J. (1985): A practical tracer gas method to determine ventilation in greenhouses. J. agric. Engng. Res. 31: 309-319

[51] Nörtersheuser, P. (1993): Aufbau von Modellen zur Beschreibung des Verhaltens von Pflanzenschutzmitteln im Boden und Anwendung am Beispiel des Herbizids Quinmerac. Dissertation Technische Universität Braunschweig, Institut für Geographie und Geoökologie 
[52] VAN'T OOSTER, A. (1994): Using natural ventilation theory and dynamic heat balance modelling for real time prediction of ventilation rates in naturally ventilated livestock houses. AgEng '94, Int. Conf. Agric. Engng., Milano, Italy, Report No. 94-C-012

[53] Paul, W. Und Jahns, G. (1988): Abschätzung des zeitlichen Verlaufs von Stoffgehalten in den Kompartimenten der Umwelt. Grundlagen der Landtechnik 38(1): 9-21

[54] Peng, S.-H. (2000): Some relations revisited in tracer gas analyses using numerical methods. In: Roomvent 2000, Ventilation for health and sustainable environment, Proceedings of the 7th international conference on air distribution in rooms (Vol I), 9 - 12 July, Reading, United Kingdom: 229-234

[55] Perić, M. (2000): Konzepte und Methoden zur Diskretisierung: Finite-Volumen-Methode. In: Numerische Methoden zur Berechnung von Strömungs- und Wärmeübertragungsproblemen, Kurzlehrgang NUMET 2000, Universität Erlangen-Nürnberg, 27.-30. März

[56] PERIĆ, M. (2000): Persönliche Mitteilung.

[57] Press, W.H.; Teukolsky, S.A.; Vetterling, W.T.; Flannery, B.P. (1992): Numerical recipes in $\mathrm{C}$ : the art of scientific computing.

[58] Raftschen, W. (1988): Was ist Lüftungseffektivität? Klima Kälte Heizung (Ki), Fachzeitschrift für technische Gebäudeausrüstung: Klima, Kälte Lüftung, Heizung, Energie Heft 5-8: 3-18, C.F. Müller Verlag, Karlsruhe

[59] RaAtschen, W. (1995): Tracergasmessungen in der Gebäudetechnik. Gesundheits-Ingenieur (gi) 116, Heft 2: 78-87 und Heft 3: 129-138, Oldenbourg Verlag, München

[60] RaAtschen, W. (1999): Persönliche Mitteilung.

[61] Rechenbach, T. (2000): Grundlagen zur Entwicklung eines Sensor-Arrays auf Schwingquarz-Basis für biogene Emissionen. Dissertation, Institut für Landtechnik, Rheinische Friedrich-Wilhelms-Universität Bonn

[62] Richter, O. (1985): Simulation des Verhaltens ökologischer Systeme: mathematische Methoden und Modelle. VCH Verlagsgesellschaft, Weinheim

[63] Sandberg, M. Und Sjøberg, M. (1983): The use of moments for assessing air quality in ventilated rooms. Build. and Environ. 18(4): 181-197

[64] SCHRÖDER, O. (1999): Persönliche Unterlagen. 
[65] Seedorf, J.; Hartung, J.; Hinz, T. (1995): Luftgetragene Verunreinigungen in und aus Rinderställen. VDI-Bericht Nr. 1211: 223-226, VDI-Verlag, Düsseldorf

[66] SEIPELT, F. (1999): Quantifizierung und Bewertung gasförmiger Emissionen aus frei gelüfteten Milchviehställen mit Trauf-First-Lüftung. Dissertation am Forschungs- und Studienzentrum für Veredelungswirtschaft Weser / Ems, Vechta, VDI-MEG Schrift 336, Cuvillier Verlag, Göttingen

[67] Stegbauer, B. (1996): Eignungstest verschiedener Ammoniakmessgeräte auf ihre Einsatztauglichkeit für Langzeitmessungen in Ställen. Diplomarbeit Technische Universität München, Institut für Landtechnik Freising-Weihenstephan, Selbstverlag

[68] Svidt, K.; BJerg, B.; Morsing, S.; Zhang, G. (1998): Modelling of air flow through a slatted floor by CFD. AgEng Oslo '98, Paper 98-B-038

[69] VARGA, L.P. AND FAlls, C.P. (1972): Continuous system models of oxygen depletion in a eutrophic reservoir. Enviromental Science and Technology 6(2): 135-142

[70] VDI-RichtLINIE 4300, BLATt 7 (2000): Messen von Innenraumluftverunreinigungen. Bestimmung der Luftwechselzahl in Innenräumen. (Vorentwurf), VDI-Verlag GmbH, Düsseldorf

[71] WEI, J. (1977): Compartmental and systemic level-times in a multicompartment system. Industrial Engineering Chemicals, Fundamentals 16(1): 19-21

[72] Yoshida, K.; Shigeoka, T.; Yamauchi, F. (1983): Non-steady-state equilibrium model for the preliminary prediction of the fate of chemicals in the environment. Ecotoxicology and Environmental Safety 7: 179-190

[73] Zenger, A.; Rühling, A.; BÄChlin, W.; Lohmeyer, A. (1994): Tracergasuntersuchungen zur naturgetreuen Simulation von atmosphärischen Transport- und Mischungsvorgängen im Rahmen von Umweltverträglichkeitsprüfungen. Staub - Reinhaltung der Luft 54: 51-54

[74] Zhang, Y. Und BARber, E.M. (1995): Air leakage and ventilation effectiveness for confinement livestock housing. Transactions of the ASAE 38(5): 1501-1504

[75] ZuRmühl, R. Und FALK, S. (1984): Matrizen und ihre Anwendungen. Teil 1: Grundlagen, Springer Verlag, Berlin 
10 Anhang 
Tabelle 7: Errechnete Luftvolumenströme des Systems $\left[\frac{m^{3}}{s}\right]$ für einen Zeitraum von 31 Sekunden für die durchgeführten Varianten der Modellversuche mit 9 bzw. 25 Kompartimenten. $v=1 \mathrm{~m} / \mathrm{s}$ jeweils im Einlass sowie $R e=13.000$.

\begin{tabular}{|c|c|c|c|c|}
\hline Zeitschritt & \multicolumn{4}{|c|}{ Modellversuch } \\
\hline & 9 & 25 & $\overrightarrow{\vec{b}}$ & 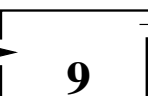 \\
\hline \multicolumn{5}{|l|}{$\mathrm{Nr}$. } \\
\hline 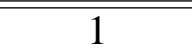 & 0,2081 & 0,2000 & 0,1983 & 0,2025 \\
\hline 2 & 0,2064 & 0,1939 & 0,2022 & 0,2003 \\
\hline 3 & 0,2094 & 0,2058 & 0,2019 & 0,2019 \\
\hline 4 & 0,2011 & 0,1939 & 0,2006 & 0,2006 \\
\hline 5 & 0,2064 & 0,1969 & 0,2006 & 0,1994 \\
\hline 6 & 0,2044 & 0,1967 & 0,1983 & 0,2022 \\
\hline 7 & 0,2019 & 0,1950 & 0,2039 & 0,1972 \\
\hline 8 & 0,2078 & 0,1919 & 0,1981 & 0,2003 \\
\hline 9 & 0,1967 & 0,1972 & 0,1997 & 0,2008 \\
\hline 10 & 0,2086 & 0,1981 & 0,1989 & 0,2000 \\
\hline 11 & 0,2044 & 0,1961 & 0,2017 & 0,2025 \\
\hline 12 & 0,1958 & 0,1933 & 0,2000 & 0,1972 \\
\hline 13 & 0,1975 & 0,1908 & 0,1994 & 0,2028 \\
\hline 14 & 0,2033 & 0,1892 & 0,2036 & 0,1989 \\
\hline 15 & 0,2039 & 0,1878 & 0,1986 & 0,2006 \\
\hline 16 & 0,2031 & 0,1869 & 0,2006 & 0,2014 \\
\hline 17 & 0,2061 & 0,1867 & 0,1992 & 0,1997 \\
\hline 18 & 0,2036 & 0,1858 & 0,2003 & 0,2025 \\
\hline 19 & 0,2086 & 0,1819 & 0,2028 & 0,2019 \\
\hline 20 & 0,2072 & 0,1819 & 0,2008 & 0,2025 \\
\hline 21 & 0,2008 & 0,1825 & 0,2031 & 0,2003 \\
\hline 22 & 0,2064 & 0,1831 & 0,1989 & 0,2022 \\
\hline 23 & 0,2069 & 0,1847 & 0,1989 & 0,1992 \\
\hline 24 & 0,2078 & 0,1875 & 0,2011 & 0,1978 \\
\hline 25 & 0,2033 & 0,1819 & 0,1997 & 0,2025 \\
\hline 26 & 0,2019 & 0,1869 & 0,1989 & 0,1953 \\
\hline 27 & 0,1997 & 0,1933 & 0,2022 & 0,2003 \\
\hline 28 & 0,2094 & 0,2006 & 0,2000 & 0,2003 \\
\hline 29 & 0,1972 & 0,2072 & 0,2022 & 0,2008 \\
\hline 30 & 0,2033 & 0,2064 & 0,2033 & 0,2011 \\
\hline 31 & 0,2089 & 0,2092 & 0,2008 & 0,1975 \\
\hline
\end{tabular}


Tabelle 8: Errechnete Luftvolumenströme des Systems $\left[\frac{m^{3}}{h}\right]$ für einen Zeitraum von 32 bzw. 31 Sekunden für die durchgeführten Feldversuche (jeweils 1. Versuch) in Faulenhorst bzw. Rothenstadt.

\begin{tabular}{|c|c|c|}
\hline Zeitschritt & Faulenhorst & Rothenstadt \\
\hline 1 & $\overline{130.170}$ & 386.668 \\
\hline 2 & 130.105 & 365.385 \\
\hline 3 & 129.994 & 378.783 \\
\hline 4 & 129.976 & 369.043 \\
\hline 5 & 129.967 & 404.271 \\
\hline 6 & 129.924 & 368.621 \\
\hline 7 & 129.861 & 367.855 \\
\hline 8 & 129.797 & 352.413 \\
\hline 9 & 129.832 & 345.877 \\
\hline 10 & 129.837 & 346.135 \\
\hline 11 & 129.867 & 324.230 \\
\hline 12 & 129.901 & 329.317 \\
\hline 13 & 129.876 & 339.684 \\
\hline 14 & 129.872 & 351.492 \\
\hline 15 & 129.854 & 306.406 \\
\hline 16 & 129.818 & 335.324 \\
\hline 17 & 129.829 & 337.957 \\
\hline 18 & 129.935 & 321.405 \\
\hline 19 & 129.975 & 320.409 \\
\hline 20 & 129.940 & 361.308 \\
\hline 21 & 129.707 & 370.703 \\
\hline 22 & 129.694 & 378.869 \\
\hline 23 & 129.708 & 384.728 \\
\hline 24 & 129.707 & 390.083 \\
\hline 25 & 129.761 & 410.667 \\
\hline 26 & 129.846 & 375.840 \\
\hline 27 & 129.979 & 358.652 \\
\hline 28 & 129.897 & 346.308 \\
\hline 29 & 129.954 & 392.088 \\
\hline 30 & 129.867 & 360.005 \\
\hline 31 & 129.836 & 374.379 \\
\hline 32 & 129.974 & - \\
\hline
\end{tabular}


Tabelle 9: Höhe der aufgezeichneten Tracerkonzentrationsmesswerte (=Ausgangswerte) an 9 Sensoren über den gesamten Versuchszeitraum zur Berechnung des Luftvolumenstromes im Modellversuch mit 9 Kompartimenten. Nach vollständiger Ausbildung des Strömungsfeldes (140 s) wird der Tracer $\left(\mathrm{NH}_{3}\right)$ schlagartig zugesetzt und die Tracerkonzentrationsmessung gestartet.

\begin{tabular}{|c|c|c|c|c|c|c|c|c|c|}
\hline it & 1 & 2 & 3 & ${ }^{2} 4$ & 5 & $P_{6}$ & $\mathbf{P}_{7}$ & 18 & \\
\hline 1 & 0998 & 9298 & 9298 & 98 & 9298 & 9298 & 9298 & 9298 & 000 \\
\hline & 9265 & 298 & & & & 98 & 98 & 98 & 0298 \\
\hline & 8439 & 296 & 88 & & 98 & 98 & 298 & 8 & 9298 \\
\hline & & 2220 & & & & & & & 0208 \\
\hline & 0286 & 13,8650 & & & & & & & 0208 \\
\hline & 12,5177 & 176 & 3 & & & 8 & 13,9298 & & 130008 \\
\hline & 827 & & & & & & & & \\
\hline & & & & & & & & & \\
\hline & & 12,5080 & & & & & & & \\
\hline & & 12,0329 & & & & & & & \\
\hline & 2135 & 2 & 128 & & & & & & \\
\hline & 7 & 11,1391 & 8 & & & & & & \\
\hline & ,9954 & 10,7998 & 84 & & & & & & \\
\hline & 10,9253 & 10,5265 & 11,0472 & & & & & & \\
\hline & 18724 & 3088 & 10,4812 & & & & & & \\
\hline 3 &, 8324 & 10,1362 & 9,9 & & & & & & \\
\hline 5 & 10,8020 & 9,9992 & 9,5491 & & & & & & 13 \\
\hline 7 & 788 & 9,8902 & 9,1864 & & & & & & \\
\hline 9 & 610 & 9,8034 & 8,8 & & 13 & 83 & & & \\
\hline 2 & 4 & 30 & 8,6 & 13,9134 & 13 & 34 & 8 & 15 & 32871 \\
\hline 1,4 & ) & 9 & 7 & 13,9114 & & 57 & & 42 & 130341 \\
\hline 4,6 & 10,7291 & 06325 & 82600 & 13,9090 & 12 & 80 & 13 & 42 & 7450 \\
\hline & 10,7230 & 2 & 81 & 13,9064 & 5 & & & & 12,4302 \\
\hline & 10,7185 & 9,5648 & 8,0 & & 12 & & 13,9150 & 12 & $1210 \mathrm{~m}$ \\
\hline & 10,7152 & 9,5395 & 7,9 & & & & & 5 & 11,7662 \\
\hline & & & 7,8 & & ) & & & & 111270 \\
\hline & & & 7,8 & & 7 & & & & 11,1202 \\
\hline & & & & & & & & & 10 \\
\hline 6,1 & & & & & & & & & \\
\hline 63 & & & 7,6 & & & & & & 10,289 \\
\hline ,6 & & & 7,6 & & & & & & 10,05 \\
\hline 8 & & 9,4428 & 7,6 & & 129147 & & & & 9,85 \\
\hline, 0 & & 9,4350 & 7,6 & & 128 & 9,0 & & & 9,0 \\
\hline 72 & 24 & 04278 & 75917 & 88 & 12788 & 90070 & 96 & 3352 & 050 \\
\hline
\end{tabular}


$\begin{array}{llllllllll}\text { Zeit } & \mathrm{P}_{1} & \mathrm{P}_{2} & \mathrm{P}_{3} & \mathrm{P}_{4} & \mathrm{P}_{5} & \mathrm{P}_{6} & \mathrm{P}_{7} & \mathrm{P}_{8} & \mathrm{P}_{9}\end{array}$

$\begin{array}{llllllllll}\mathbf{1 4 7 , 4} & 10,6988 & 9,4209 & 7,5779 & 13,7215 & 12,7255 & 8,9666 & 13,1682 & 11,8314 & 9,3619\end{array}$

$\begin{array}{llllllllll}\mathbf{1 4 7 , 6} & 10,6937 & 9,4141 & 7,5661 & 13,6716 & 12,6625 & 8,9145 & 13,0133 & 11,6324 & 9,2356\end{array}$

$\begin{array}{llllllllll}\mathbf{1 4 7 9} & 10,6866 & 9,4070 & 7,5560 & 13,6125 & 12,5994 & 8,8694 & 12,8467 & 11,4399 & 9,1249\end{array}$

$\begin{array}{llllllllll}\mathbf{1 4 8 , 1} & 10,6769 & 9,3994 & 7,5472 & 13,5435 & 12,5360 & 8,8303 & 12,6709 & 11,2557 & 9,0281\end{array}$

$\begin{array}{llllllllll}\mathbf{1 4 8 , 3} & 10,6641 & 9,3911 & 7,5393 & 13,4644 & 12,4722 & 8,7961 & 12,4880 & 11,0807 & 8,9433\end{array}$

$\begin{array}{lllllllllll}\mathbf{1 4 8 , 5} & 10,6474 & 9,3816 & 7,5321 & 13,3751 & 12,4082 & 8,7661 & 12,3006 & 10,9157 & 8,8691\end{array}$

$\begin{array}{llllllllll}\mathbf{1 4 8 , 7} & 10,6262 & 9,3705 & 7,5253 & 13,2758 & 12,3437 & 8,7396 & 12,1109 & 10,7611 & 8,8041\end{array}$

$\begin{array}{llllllllll}\mathbf{1 4 8 , 8} & 10,6164 & 9,3656 & 7,5227 & 13,2334 & 12,3176 & 8,7298 & 12,0348 & 10,7022 & 8,7804\end{array}$

$\begin{array}{llllllllll}\mathbf{1 4 9 , 0} & 10,5909 & 9,3533 & 7,5167 & 13,1325 & 12,2588 & 8,7093 & 11,8642 & 10,5759 & 8,7315\end{array}$

$\begin{array}{llllllllll}\mathbf{1 4 9 , 3} & 10,5571 & 9,3373 & 7,5098 & 13,0123 & 12,1928 & 8,6886 & 11,6772 & 10,4451 & 8,6833\end{array}$

$\begin{array}{llllllllll}\mathbf{1 4 9 , 5} & 10,5170 & 9,3185 & 7,5024 & 12,8845 & 12,1260 & 8,6695 & 11,4940 & 10,3242 & 8,6407\end{array}$

$\begin{array}{llllllllll}\mathbf{1 4 9 , 7} & 10,4702 & 9,2965 & 7,4943 & 12,7499 & 12,0586 & 8,6517 & 11,3160 & 10,2127 & 8,6029\end{array}$

$\begin{array}{llllllllll}\mathbf{1 4 9 , 9} & 10,4163 & 9,2708 & 7,4851 & 12,6098 & 11,9901 & 8,6349 & 11,1445 & 10,1101 & 8,5692\end{array}$

$\begin{array}{llllllllll}\mathbf{1 5 0 , 1} & 10,3553 & 9,2412 & 7,4745 & 12,4652 & 11,9207 & 8,6185 & 10,9801 & 10,0158 & 8,5390\end{array}$

$\begin{array}{llllllllll}\mathbf{1 5 0 , 4} & 10,2871 & 9,2072 & 7,4622 & 12,3175 & 11,8503 & 8,6023 & 10,8234 & 9,9290 & 8,5117\end{array}$

$\begin{array}{llllllllll}\mathbf{1 5 0 , 6} & 10,2120 & 9,1687 & 7,4478 & 12,1677 & 11,7789 & 8,5860 & 10,6749 & 9,8493 & 8,4867\end{array}$

$\begin{array}{llllllllll}\mathbf{1 5 0 , 8} & 10,1303 & 9,1255 & 7,4312 & 12,0172 & 11,7066 & 8,5692 & 10,5347 & 9,7759 & 8,4636\end{array}$

$\begin{array}{llllllllll}\mathbf{1 5 1 , 0} & 10,0423 & 9,0773 & 7,4118 & 11,8668 & 11,6332 & 8,5516 & 10,4029 & 9,7083 & 8,4420\end{array}$

$\begin{array}{llllllllll}\mathbf{1 5 1 , 2} & 9,9486 & 9,0243 & 7,3895 & 11,7176 & 11,5588 & 8,5328 & 10,2793 & 9,6459 & 8,4215\end{array}$

$\begin{array}{llllllllll}\mathbf{1 5 1 , 4} & 9,8499 & 8,9664 & 7,3640 & 11,5704 & 11,4835 & 8,5126 & 10,1637 & 9,5880 & 8,4017\end{array}$

$\begin{array}{llllllllll}\mathbf{1 5 1 , 7} & 9,7470 & 8,9038 & 7,3351 & 11,4261 & 11,4072 & 8,4907 & 10,0560 & 9,5342 & 8,3822\end{array}$

$\begin{array}{llllllllll}\mathbf{1 5 1 , 9} & 9,6405 & 8,8366 & 7,3025 & 11,2852 & 11,3300 & 8,4667 & 9,9556 & 9,4839 & 8,3627\end{array}$

$\begin{array}{llllllllll}\mathbf{1 5 2 , 1} & 9,5312 & 8,7652 & 7,2662 & 11,1484 & 11,2520 & 8,4404 & 9,8622 & 9,4367 & 8,3427\end{array}$

$\begin{array}{llllllllll}\mathbf{1 5 2 , 3} & 9,4200 & 8,6899 & 7,2261 & 11,0160 & 11,1732 & 8,4115 & 9,7753 & 9,3921 & 8,3221\end{array}$

$\begin{array}{llllllllll}\mathbf{1 5 2 , 5} & 9,3077 & 8,6112 & 7,1823 & 10,8885 & 11,0937 & 8,3798 & 9,6947 & 9,3496 & 8,3005\end{array}$

$\begin{array}{llllllllll}\mathbf{1 5 2 , 8} & 9,1949 & 8,5294 & 7,1347 & 10,7659 & 11,0135 & 8,3452 & 9,6197 & 9,3089 & 8,2776\end{array}$

$\begin{array}{llllllllll}\mathbf{1 5 3 , 0} & 9,0825 & 8,4450 & 7,0835 & 10,6486 & 10,9328 & 8,3074 & 9,5499 & 9,2697 & 8,2530\end{array}$

$\begin{array}{llllllllll}\mathbf{1 5 3 , 2} & 8,9710 & 8,3586 & 7,0289 & 10,5364 & 10,8517 & 8,2663 & 9,4848 & 9,2314 & 8,2265\end{array}$

$\begin{array}{llllllllll}\mathbf{1 5 3 , 4} & 8,8611 & 8,2707 & 6,9712 & 10,4295 & 10,7702 & 8,2220 & 9,4241 & 9,1939 & 8,1979\end{array}$

$\begin{array}{llllllllll}\mathbf{1 5 3 , 6} & 8,7533 & 8,1818 & 6,9105 & 10,3278 & 10,6885 & 8,1743 & 9,3671 & 9,1568 & 8,1670\end{array}$

$\begin{array}{llllllllll}\mathbf{1 5 3 , 8} & 8,6481 & 8,0924 & 6,8473 & 10,2311 & 10,6066 & 8,1233 & 9,3136 & 9,1197 & 8,1335\end{array}$

$\begin{array}{llllllllll}\mathbf{1 5 4 , 1} & 8,5457 & 8,0029 & 6,7819 & 10,1394 & 10,5247 & 8,0690 & 9,2631 & 9,0824 & 8,0972\end{array}$

$\begin{array}{llllllllll}\mathbf{1 5 4 , 3} & 8,4466 & 7,9139 & 6,7147 & 10,0523 & 10,4427 & 8,0117 & 9,2152 & 9,0446 & 8,0582\end{array}$

$\begin{array}{llllllllll}\mathbf{1 5 4 , 5} & 8,3509 & 7,8257 & 6,6460 & 9,9696 & 10,3609 & 7,9514 & 9,1695 & 9,0061 & 8,0161\end{array}$

$\begin{array}{llllllllll}\mathbf{1 5 4}, 7 & 8,2588 & 7,7388 & 6,5762 & 9,8912 & 10,2794 & 7,8884 & 9,1256 & 8,9666 & 7,9712\end{array}$

$\begin{array}{llllllllll}\mathbf{1 5 4 , 9} & 8,1705 & 7,6533 & 6,5058 & 9,8167 & 10,1981 & 7,8230 & 9,0832 & 8,9259 & 7,9232\end{array}$

$\begin{array}{llllllllll}\mathbf{1 5 5 , 1} & 8,0859 & 7,5697 & 6,4352 & 9,7459 & 10,1172 & 7,7554 & 9,0419 & 8,8838 & 7,8723\end{array}$

$\begin{array}{llllllllll}\mathbf{1 5 5 , 4} & 8,0052 & 7,4882 & 6,3645 & 9,6784 & 10,0368 & 7,6858 & 9,0014 & 8,8402 & 7,8186\end{array}$ 


\begin{tabular}{|c|c|c|c|c|c|c|c|c|}
\hline $\mathrm{P}_{1}$ & $\mathrm{P}_{2}$ & $\mathrm{P}_{3}$ & $\mathrm{P}_{4}$ & $P_{5}$ & $P_{6}$ & $\mathrm{P}_{7}$ & $\mathrm{P}_{8}$ & $\mathrm{P}_{9}$ \\
\hline 7,9282 & 7,4090 & 6,2943 & 9,6140 & 9,9570 & 7,6147 & 8,9613 & 8,7950 & 7,7622 \\
\hline 7,8550 & 7,3321 & 6,2248 & 9,5524 & 9,8777 & 7,5423 & 9214 & (d) & 7,7033 \\
\hline 7,7853 & 7,2578 & 6,1564 & 9,4932 & 9,7992 & 7,4690 & 8,8814 & 8,6991 & 7,6420 \\
\hline 7,7190 & 7,1862 & 6,0891 & 9,4362 & 9,7214 & 7,3951 & 8,8410 & 8,6483 & 7,5786 \\
\hline 7,6560 & 7,1171 & 6,0233 & 9,3811 & 9,6443 & 7,3210 & 7999 & 8,5956 & 7,5134 \\
\hline 7,5962 & 7,0508 & 5,9592 & 3276 & 9,5681 & 7,2469 & 7581 & 409 & 7,4465 \\
\hline 7,5392 & 6,9870 & 5,8968 & 9,2755 & 9,4927 & 7,1731 & 7152 & 4844 & 7,3783 \\
\hline 7,4850 & 6,9259 & 5,8363 & 9,2243 & 9,4182 & 7,1000 & 8,6712 & 8,4260 & 7,3092 \\
\hline 7,4332 & 6,8673 & 5,7778 & 9,1740 & 9,3447 & 7,0277 & 8,6258 & 8,3659 & 7,2393 \\
\hline 7,3838 & 6,8111 & 5,7213 & 9,1243 & 9,2720 & 6,9564 & 8,5789 & 8,3041 & 7,1690 \\
\hline 7,3364 & 6,7572 & 5,6669 & 9,0749 & 9,2003 & 6,8865 & 8,5305 & 8,2409 & 7,0985 \\
\hline 7,2908 & 6,7055 & 5,6146 & 9,0256 & 9,1294 & 6,8179 & 4805 & 1763 & 7,0281 \\
\hline 7,2468 & 6,6559 & 5,5642 & 762 & 9,0596 & 6,7510 & 89 & 105 & 6,9581 \\
\hline 7,2042 & 6,6081 & 5,5159 & 8,9266 & 8,9906 & 6,6857 & 3756 & 8,0437 & 6,8887 \\
\hline 7,1628 & 6,5622 & 5,4695 & 8,8766 & 8,9225 & 6,6221 & 8,3207 & 7,9760 & 6,8201 \\
\hline 7,1224 & 6,5179 & 5,4250 & 8,8260 & 8,8553 & 6,5605 & 8,2642 & 7,9078 & 6,7526 \\
\hline 7,0827 & 6,4750 & 5,3823 & 8,7748 & 8,7889 & 6,5007 & 8,2062 & 7,8390 & 6,6861 \\
\hline 426 & 6 & 5 & 8 & 34 & 428 & 167 & 7,7700 & 6,6210 \\
\hline 6 & 6 & 5 , & 0 & 37 & 8 & 9 & 10 & 74 \\
\hline 6,9666 & 0 & 5,2640 & 8,6161 & 8, & 6,3326 & 39 & 7,6320 & 6,4952 \\
\hline 6,9282 & 6,3151 & 5,2274 & 8,5613 & 8,5316 & 6,2803 & 7,9608 & 7,5634 & 6,4347 \\
\hline 6,8898 & 6,2772 & 5,1921 & 8,5055 & 8,4691 & 6,2297 & 7,8968 & 7,4952 & 6,3759 \\
\hline 6,8511 & 6,2399 & 5,1579 & 8,4488 & 8,4073 & 6,1807 & 7,8321 & 7,4275 & 6,3187 \\
\hline 6,8122 & 6,2031 & 5,1247 & 8,3911 & 8,3461 & 6,1334 & 7,7667 & 7,3606 & 6,2633 \\
\hline 6,7729 & 6,1665 & 5,0924 & 8,3324 & 8,2856 & 6,0876 & 7,7009 & 7,2946 & 6,2095 \\
\hline 7330 & 6,1301 & 5,0608 & 29 & 56 & 33 & 47 & 95 & 574 \\
\hline 6926 & 6,0939 & 5,0300 & 8,2125 & 8,1661 & 6,0002 & 85 & 7,1654 & 6,1070 \\
\hline 6,6515 & 6,0576 & 4,9996 & 8,1513 & 8,1072 & 5,9585 & 7,5023 & 7,1025 & 6,0581 \\
\hline 6,6098 & 6,0212 & 4,9697 & 8,0895 & 8,0487 & 5,9178 & 7,4363 & 7,0407 & 6,0107 \\
\hline 6,5673 & 5,9846 & 4,9401 & 8,0271 & 7,9906 & 5,8781 & 7,3706 & 6,9802 & 5,9648 \\
\hline 6,5242 & 5,9478 & 4,9108 & 7,9642 & 7,9330 & 5,8394 & 7,3053 & 6,9210 & 5,9203 \\
\hline 6,4803 & 5,9106 & 4,8816 & 7,9008 & 7,8758 & 5,8014 & 7,2407 & 6,8630 & 5,8771 \\
\hline 6,4358 & 5,8731 & 4,8524 & 7,8373 & 7,8189 & 5,7641 & 7,1767 & 6,8063 & 5,8351 \\
\hline 6,3905 & 5,8352 & 4,8232 & 7,7735 & 7,7624 & 5,7275 & 7,1135 & 6,7509 & 5,7943 \\
\hline 6,3447 & 5,7970 & 4,7940 & 7,7097 & 7,7062 & 5,6913 & 7,0513 & 6,6967 & 5,7544 \\
\hline 6,2982 & 5,7582 & 4,7646 & 7,6458 & 7,6503 & 5,6555 & 6,9900 & 6,6438 & 5,7155 \\
\hline 6,2512 & 5,7191 & 4,7350 & 7,5822 & 7,5947 & 5,6200 & 6,9297 & 6,5922 & 5,6774 \\
\hline 6,2038 & 5,6795 & 4,7052 & 7,5188 & 7,5394 & 5,5847 & 6,8704 & 6,5416 & 400 \\
\hline
\end{tabular}




\begin{tabular}{|c|c|c|c|c|c|c|c|c|c|}
\hline it & $P_{1}$ & $P_{2}$ & $P_{3}$ & $P_{4}$ & $P_{5}$ & $P_{6}$ & $P_{7}$ & $\mathrm{P}_{8}$ & $P_{9}$ \\
\hline 639 & 1559 & 5,6395 & 4,6751 & 7,4557 & 7,4843 & 5,5496 & 6,8123 & 6,4923 & 5,6033 \\
\hline 1 & 077 & 9992 & 4,6447 & 7,3931 & 7,4296 & 145 & 6,7554 & 4439 & ,5671 \\
\hline 4,3 & 593 & 584 & 4,6140 & 3309 & 3750 & 794 & 6995 & 6,3966 & 53 \\
\hline & 107 & 73 & 4,5830 & 2694 & 08 & 443 & 49 & in & 4060 \\
\hline & 619 & 60 & 4,5516 & 2085 & 68 & 991 & 914 & & 160 \\
\hline & 9132 & 5,4344 & 4,5199 & 7,1483 & 2130 & 5,3738 & 390 & $0, \Delta 01$ & 20 \\
\hline 2 & 5,8645 & 5,3926 & 4,4880 & 7,0890 & 7,1 & 383 & 77 & 6,2162 & 4 \\
\hline 4 & 5,8159 & 5,3506 & 4,4557 & 7,0304 & 7,1063 & 5,3026 & 376 & 729 & 568 \\
\hline 6 & 5,7676 & 5,3086 & 4,4231 & 6,9727 & 7,0533 & 5,2667 & 8884 & 1303 & 5,3222 \\
\hline 5,8 & 195 & 5,2665 & OB & 9158 & 7,0006 & 306 & 3 & 82 & 5,2876 \\
\hline & 5,6718 & 5,2244 & 4,3573 & 999 & 82 & 942 & & 167 & 5050 \\
\hline & 5,6244 & 5,1824 & 4,3242 & 8048 & 1 & 577 & 9 & 6,0055 & $\sqrt{, 210}$ \\
\hline & 5,5775 & 5,1405 & 4,2908 & 6,7507 & 142 & 5,1209 & 5 & 47 & 0.35 \\
\hline 7 & 5,5311 & 5,0988 & 4,2574 & 6,6975 & 6,7927 & 5,0839 & 6,1569 & 5,9242 & 5,1482 \\
\hline 9 & 5,4853 & 5,0573 & 4,2240 & 6,6451 & 115 & 5,0467 & 31 & 5,8840 & 130 \\
\hline 871 & 5,4400 & 50 & 5 & 7 & & & 9 & & 76 \\
\hline 7,4 & 5,3953 & 4,9752 & & & & & & & 5,0420 \\
\hline 7,6 & 513 & 4,9346 & 4,1236 & 34 & & 343 & & & 5,0063 \\
\hline 8 & 5,3079 & 4,8945 & 4,0903 & 6,4445 & 8 & 4,8967 & 7 & 47 & 4,9704 \\
\hline 0 & 5,2652 & 4,8547 & 4,0572 & 6,3964 & ,4903 & 4,8590 & $\delta$ & 5,6 & 4,934 \\
\hline 3,2 & 5,2232 & 4,8154 & 4,0242 & 6,3490 & 6,4411 & 4,8213 & 5,8622 & 5,6456 & 4,8981 \\
\hline, 4 & 1818 & 4,7765 & 3,9915 & 6,3023 & 6,3923 & 4,7836 & 5,8219 & 5,6061 & 4,8618 \\
\hline 68,7 & 2 & 4,73 & 90 & 63 & 88 & 4,7 & 9 & 6 & 4,8254 \\
\hline & ? & & 3,9268 & 10 & & 36 & 22 & 5,5 & 000 \\
\hline & 5,0618 & 4, & & 2 & & 4, & 7 & 7 & 4,7524 \\
\hline S & 5,0231 & 4,6262 & 3,8634 & 0,1220 & 07 & 4,6341 & 5,6633 & 5,4482 & 139 \\
\hline 9,5 & 4,9850 & 4,5898 & 3,8322 & 6,0782 & 6,1538 & 4,5972 & 5,6241 & 5,4087 & 4,6794 \\
\hline 88 & 4,9475 & 4,5541 & 3,8013 & 6,0350 & 6,1073 & 4,5605 & 5,5850 & 5,3692 & 4,6429 \\
\hline a & 4,9106 & 4,5188 & 3,7709 & 5,9921 & 6,0612 & 4,5241 & 460 & 5,3 & 4,6066 \\
\hline & 4,8742 & 4,4841 & 3,7408 & 5,9497 & 0154 & 4,4880 & 500 & 2 & 4,5703 \\
\hline 0,4 & 4,8384 & 4,4498 & 3,7112 & 5,9076 & 5,9700 & 4,4521 & 5,4682 & 5,2508 & דב \\
\hline 6 & 4,8031 & 4,4161 & 3,6819 & 5,8658 & 5,9251 & 4,4167 & 5,4294 & 5,2114 & 4,4984 \\
\hline 8 & 4,7682 & 4,3828 & 3,6531 & 5,8243 & 5,8805 & 4,3816 & 5,3906 & 5,1721 & 4,4627 \\
\hline 71,1 & 4,7338 & 4,3500 & 3,6247 & 5,7831 & 5,8363 & 4,3469 & 5,3518 & 5,1329 & 4,4273 \\
\hline 2 & 4,6998 & $4,31^{\circ}$ & & & & 4,3126 & 5,3 & 5,0938 & 4,3921 \\
\hline 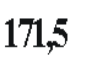 & 4,6662 & 4,2857 & 3,5690 & 5,7013 & 5,7491 & 4,2786 & 5,2744 & 5,0548 & 4,35 \\
\hline 1,7 & 4,6329 & 4,2542 & 3,5418 & 5,6607 & 5,7060 & 4,2451 & 5,2357 & 5,0160 & 4,322 \\
\hline 719 & 4,5999 & 42230 & 35150 & 5,6203 & 5,6633 & 4,2120 & 51971 & 4,9774 & 178 \\
\hline
\end{tabular}


$\begin{array}{llllllllll}\text { Zeit } & \mathrm{P}_{1} & \mathrm{P}_{2} & \mathrm{P}_{3} & \mathrm{P}_{4} & \mathrm{P}_{5} & \mathrm{P}_{6} & \mathrm{P}_{7} & \mathrm{P}_{8} & \mathrm{P}_{9}\end{array}$

$\begin{array}{llllllllll}\mathbf{1 7 2 , 2} & 4,5672 & 4,1922 & 3,4885 & 5,5800 & 5,6210 & 4,1793 & 5,1585 & 4,9389 & 4,2545\end{array}$

$\begin{array}{llllllllll}\mathbf{1 7 2 , 4} & 4,5348 & 4,1618 & 3,4624 & 5,5399 & 5,5790 & 4,1471 & 5,1200 & 4,9007 & 4,2209\end{array}$

$\begin{array}{llllllllll}172,6 & 4,5026 & 4,1317 & 3,4367 & 5,4999 & 5,5374 & 4,1152 & 5,0816 & 4,8627 & 4,1878\end{array}$

$\begin{array}{llllllllll}\mathbf{1 7 2 , 8} & 4,4707 & 4,1019 & 3,4113 & 5,4600 & 5,4961 & 4,0838 & 5,0433 & 4,8250 & 4,1550\end{array}$

$\begin{array}{llllllllll}\mathbf{1 7 3 , 0} & 4,4389 & 4,0723 & 3,3862 & 5,4203 & 5,4552 & 4,0528 & 5,0051 & 4,7875 & 4,1226\end{array}$

$\begin{array}{llllllllll}\mathbf{1 7 3 2} & 4,4073 & 4,0431 & 3,3614 & 5,3806 & 5,4146 & 4,0221 & 4,9671 & 4,7503 & 4,0905\end{array}$

$\begin{array}{llllllllll}\mathbf{1 7 3 , 5} & 4,3759 & 4,0141 & 3,3369 & 5,3411 & 5,3743 & 3,9919 & 4,9291 & 4,7134 & 4,0589\end{array}$

$\begin{array}{llllllllll}\mathbf{1 7 3 , 7} & 4,3447 & 3,9852 & 3,3127 & 5,3017 & 5,3344 & 3,9620 & 4,8914 & 4,6768 & 4,0277\end{array}$

$\begin{array}{llllllllll}\mathbf{1 7 3 , 9} & 4,3135 & 3,9567 & 3,2887 & 5,2625 & 5,2947 & 3,9325 & 4,8538 & 4,6406 & 3,9968\end{array}$

$\begin{array}{llllllllll}\mathbf{1 7 4 , 1} & 4,2825 & 3,9283 & 3,2649 & 5,2234 & 5,2554 & 3,9033 & 4,8165 & 4,6047 & 3,9664\end{array}$

$\begin{array}{llllllllll}\mathbf{1 7 4 , 3} & 4,2516 & 3,9000 & 3,2414 & 5,1844 & 5,2164 & 3,8744 & 4,7793 & 4,5691 & 3,9363\end{array}$

$\begin{array}{llllllllll}\mathbf{1 7 4 , 6} & 4,2208 & 3,8720 & 3,2181 & 5,1456 & 5,1776 & 3,8459 & 4,7424 & 4,5339 & 3,9065\end{array}$

$\begin{array}{llllllllll}\mathbf{1 7 4 , 8} & 4,1902 & 3,8441 & 3,1950 & 5,1069 & 5,1392 & 3,8177 & 4,7058 & 4,4990 & 3,8772\end{array}$

$\begin{array}{llllllllll}\mathbf{1 7 5 , 0} & 4,1596 & 3,8163 & 3,1720 & 5,0684 & 5,1011 & 3,7898 & 4,6694 & 4,4645 & 3,8482\end{array}$

$\begin{array}{llllllllll}\mathbf{1 7 5 , 2} & 4,1291 & 3,7887 & 3,1492 & 5,0301 & 5,0632 & 3,7621 & 4,6333 & 4,4304 & 3,8195\end{array}$

$\begin{array}{llllllllll}\mathbf{1 7 5 , 4} & 4,0987 & 3,7612 & 3,1266 & 4,9920 & 5,0256 & 3,7347 & 4,5974 & 4,3966 & 3,7912\end{array}$

$\begin{array}{llllllllll}\mathbf{1 7 5 , 6} & 4,0685 & 3,7338 & 3,1041 & 4,9541 & 4,9883 & 3,7076 & 4,5619 & 4,3631 & 3,7631\end{array}$

$\begin{array}{llllllllll}\mathbf{1 7 5 9 9} & 4,0383 & 3,7066 & 3,0817 & 4,9164 & 4,9512 & 3,6807 & 4,5267 & 4,3301 & 3,7354\end{array}$

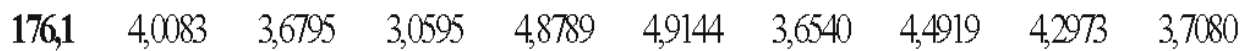

$\begin{array}{llllllllll}\mathbf{1 7 6 , 3} & 3,9783 & 3,6524 & 3,0374 & 4,8417 & 4,8779 & 3,6275 & 4,4573 & 4,2650 & 3,6808\end{array}$

$\begin{array}{llllllllll}\mathbf{1 7 6 , 5} & 3,9485 & 3,6255 & 3,0154 & 4,8047 & 4,8416 & 3,6012 & 4,4231 & 4,2329 & 3,6539\end{array}$

$\begin{array}{llllllllll}\mathbf{1 7 6 , 7} & 3,9188 & 3,5987 & 2,9934 & 4,7680 & 4,8056 & 3,5751 & 4,3892 & 4,2012 & 3,6273\end{array}$

$\begin{array}{llllllllll}176,9 & 3,8892 & 3,5721 & 2,9716 & 4,7315 & 4,7698 & 3,5492 & 4,3557 & 4,1698 & 3,6008\end{array}$

$\begin{array}{llllllllll}177,2 & 3,8598 & 3,5455 & 2,9499 & 4,6953 & 4,7343 & 3,5234 & 4,3224 & 4,1387 & 3,5746\end{array}$

$\begin{array}{llllllllll}\mathbf{1 7 7 , 4} & 3,8306 & 3,5191 & 2,9283 & 4,6594 & 4,6990 & 3,4978 & 4,2896 & 4,1080 & 3,5487\end{array}$

$\begin{array}{llllllllll}177,6 & 3,8015 & 3,4927 & 2,9068 & 4,6238 & 4,6640 & 3,4723 & 4,2570 & 4,0775 & 3,5229\end{array}$

$\begin{array}{llllllllll}\mathbf{1 7 7 , 8} & 3,7726 & 3,4666 & 2,8853 & 4,5885 & 4,6292 & 3,4470 & 4,2248 & 4,0473 & 3,4973\end{array}$

$\begin{array}{llllllllll}\mathbf{1 7 8 , 0} & 3,7438 & 3,4405 & 2,8640 & 4,5535 & 4,5946 & 3,4218 & 4,1930 & 4,0174 & 3,4718\end{array}$

$\begin{array}{llllllllll}\mathbf{1 7 8 , 3} & 3,7153 & 3,4146 & 2,8428 & 4,5188 & 4,5603 & 3,3968 & 4,1614 & 3,9878 & 3,4466\end{array}$

$\begin{array}{llllllllll}\mathbf{1 7 8 , 5} & 3,6869 & 3,3888 & 2,8216 & 4,4844 & 4,5263 & 3,3718 & 4,1302 & 3,9584 & 3,4215\end{array}$

$\begin{array}{llllllllll}\mathbf{1 7 8 , 7} & 3,6587 & 3,3632 & 2,8006 & 4,4503 & 4,4925 & 3,3470 & 4,0993 & 3,9293 & 3,3966\end{array}$

$\begin{array}{llllllllll}\mathbf{1 7 8 , 9} & 3,6298 & 3,3370 & 27792 & 4,4151 & 4,4578 & 3,3218 & 4,0672 & 3,8992 & 3,3711\end{array}$

$\begin{array}{llllllllll}179,1 & 3,6030 & 3,3124 & 27588 & 4,3831 & 4,4255 & 3,2978 & 4,0384 & 3,8717 & 3,3472\end{array}$

$\begin{array}{llllllllll}\mathbf{1 7 9 , 3} & 3,5754 & 3,2873 & 27380 & 4,3499 & 4,3924 & 3,2734 & 4,0083 & 3,8433 & 3,3226\end{array}$

$\begin{array}{lllllllllll}179,6 & 3,5481 & 3,2623 & 27174 & 4,3171 & 4,3595 & 3,2491 & 3,9786 & 3,8151 & 3,2983\end{array}$

$\begin{array}{llllllllll}\mathbf{1 7 9 , 8} & 3,5210 & 3,2375 & 2,6969 & 4,2846 & 4,3269 & 3,2249 & 3,9491 & 3,7870 & 3,2740\end{array}$

$\begin{array}{llllllllll}\mathbf{1 8 0 , 0} & 3,4942 & 3,2129 & 2,6765 & 4,2523 & 4,2945 & 3,2008 & 3,9199 & 3,7592 & 3,2499\end{array}$

$\begin{array}{llllllllll}\mathbf{1 8 0 , 2} & 3,4676 & 3,1884 & 2,6562 & 4,2204 & 4,2623 & 3,1769 & 3,8910 & 3,7316 & 3,2259\end{array}$ 


\begin{tabular}{|c|c|c|c|c|c|c|c|c|}
\hline $\mathrm{P}_{1}$ & $\mathrm{P}_{2}$ & $\mathrm{P}_{3}$ & $\mathrm{P}_{4}$ & $P_{5}$ & $\mathrm{P}_{6}$ & $\mathrm{P}_{7}$ & $\mathrm{P}_{8}$ & $P_{9}$ \\
\hline 3,4412 & 3,1642 & 2,6361 & 4,1888 & 4,2304 & 3,1531 & 3,8623 & 3,7042 & 3,2021 \\
\hline 3,4150 & 3,1401 & 26161 & 4,1574 & 4,1987 & 3,1295 & 3,8338 & 3,6769 & 3,1783 \\
\hline 3,3891 & 3,1162 & 2,5962 & 4,1264 & 4,1672 & 3,1059 & 3,8056 & 3,6498 & 3,1547 \\
\hline 3,3634 & 3,0925 & 2,5765 & 4,0956 & 4,1360 & 3,0826 & 3,7776 & 6229 & 1312 \\
\hline 3,3380 & 3,0691 &, 5569 & 4,0651 & 4,1050 & 3,0593 & 7498 & 5962 & 1079 \\
\hline 3,3128 & 3,0458 & 5375 & 4,0348 & 4,0743 & 3,0362 & 7222 & 696 & 0846 \\
\hline 3,2878 & 3,0227 & 2,5182 & 4,0049 & 4,0437 & 3,0133 & 3,6948 & 3,5432 & 0615 \\
\hline 3,2631 & 2,9998 & 2,4990 & 3,9751 & 4,0134 & 2,9905 & 3,6677 & 3,5170 & 3,0386 \\
\hline 3,2385 & 29772 & 2,4800 & 3,9457 & 3,9834 & 2,9679 & 3,6407 & 3,4909 & 3,0157 \\
\hline 3,2142 & 2,9547 & 2,4612 & 3,9164 & 3,9535 & 2,9454 & 3,6138 & 3,4649 & 29931 \\
\hline 3,1902 & 2,9324 & 2,4425 & 3,8875 & 3,9239 & 2,9231 & 5872 & 3,4392 & 29705 \\
\hline 3,1663 & 2,9103 & 2,4240 & 3,8587 & 3,8945 & 2,9010 & 608 & W & 9481 \\
\hline 3,1427 & 2,8884 & 2,4057 & 3,8302 & 3,8654 & 2,8790 & 5345 & 3,5881 & 29259 \\
\hline 3,1193 & 2,8667 & 233875 & 3,8018 & 3,8364 & 2,8572 & 3,5083 & 3,3628 & 29038 \\
\hline 3,0960 & 2,8452 & 23694 & 3,7737 & 3,8077 & 2,8356 & 3,4824 & 3,3377 & 2,8818 \\
\hline 3,0730 & 2,8239 & 23515 & 3,7458 & 3,7792 & 2,8141 & 3,4566 & 3,3127 & 2,8600 \\
\hline 0502 & 2,8028 & 23338 & 37181 & 3,7509 & 28 & 3,4310 & 3,2879 & 2,8384 \\
\hline 1275 & 27818 & 2 & $\mathrm{~J}$ & 3,7229 & 2,7717 & 55 & 32 & 169 \\
\hline 3,0051 & 27611 & 2,2988 & 3,6633 & 3,6950 & 2,7508 & 02 & 3,2387 & 27956 \\
\hline 2,9828 & 27405 & 2,2816 & 3,6362 & 3,6674 & 2,7300 & 3,3550 & 3,2144 & 27745 \\
\hline 2,9607 & 27201 & 2,2645 & 3,6093 & 3,6400 & 2,7095 & 3,3301 & 3,1902 & 27535 \\
\hline 2,9387 & 2,6998 & 22475 & 3,5825 & 3,6128 & 2,6891 & 3,3052 & 3,1663 & 27327 \\
\hline 2,9170 & 2,6797 & 2,2307 & 3,5560 & 3,5858 & 2,6688 & 3,2806 & 3,1424 & 27120 \\
\hline . & 2 & 22 & 3,5 & 3,5590 & 2 & 60 & 88 & 916 \\
\hline 8739 & 2,6401 & 2,1975 & 3,5034 & 3,5324 & 2,6289 & 3,2317 & 53 & 2,6713 \\
\hline 8527 & 2,6204 & 2,18 & 3,4774 & 3,5060 & 2,6091 & 3,2075 & 3,0720 & 26511 \\
\hline 2,8315 & 2,6010 & 2,1649 & 3,4515 & 3,4799 & 2,5896 & 3,1834 & 3,0489 & 26312 \\
\hline 2,8105 & 2,5817 & 2,1488 & 3,4258 & 3,4539 & 2,5702 & 3,1596 & 3,0259 & 26114 \\
\hline 2,7897 & 2,5625 & 2,1328 & 3,4003 & 3,4281 & 2,5510 & 3,1359 & 3,0031 & 2,5917 \\
\hline 2,7690 & 2,5435 & 21169 & 3,3749 & 3,4025 & 2 & 3,1123 & 29 & 2,5723 \\
\hline 2,7485 & 2,5246 & 2,1012 & 3,3498 & 3,377 & 2,5130 & 3,0889 & 2,95 & 25530 \\
\hline 2,7281 & 2,5059 & 20856 & 3,3248 & 3,3519 & 2,4943 & 3,0657 & 29359 & 2,5338 \\
\hline 2,7078 & 2,4873 & 2,0701 & 3,2999 & 3,3269 & 2,4757 & 3,0427 & 2,9138 & 25148 \\
\hline 2,6876 & 2,4688 & 20548 & 3,2753 & 3,3020 & 2,4572 & 3,0198 & 28919 & 24960 \\
\hline 2,6677 & 2,4505 & 20395 & 3,2508 & 3,2774 & 2,4389 & 2,9971 & 28702 & 24774 \\
\hline 2,6478 & 2,4323 & 2,0244 & 3,2264 & 3,2529 & 2,4208 & 2,9746 & 28486 & 24588 \\
\hline 2,6280 & 2,4142 & 2,0093 & 3,2023 & 3,2287 & 2,4027 & 2,9522 & 28273 & 24405 \\
\hline 2,6085 & 23962 & 1,9944 & 3,1783 & 3,2046 & 2,3849 & 2,9300 & 28061 & 2,422 \\
\hline
\end{tabular}




\begin{tabular}{|c|c|c|c|c|c|c|c|c|}
\hline$P_{1}$ & $\mathrm{P}_{2}$ & $P_{3}$ & $P_{4}$ & $P_{5}$ & $\mathrm{P}_{6}$ & $\mathrm{P}_{7}$ & $P_{8}$ & $P_{9}$ \\
\hline 2,5890 & 23784 & 1,9796 & 3,1545 & 3,1806 & 2,3671 & 2,9080 & 27050 & 2,4042 \\
\hline 2,5696 & 236007 & 1,9649 & 3,1308 & 3,1569 & 2,3495 & 2,8861 & 27642 & 23863 \\
\hline 2,5504 & 2,3431 & 1,9503 & 3,1073 & 3,1333 & 2,3321 & 8644 & 435 & 3685 \\
\hline 2,5314 & 2,3256 & 1,9358 & 3,0840 & 3,1099 & 2,3147 & 2,8429 & 27230 & 23509 \\
\hline 2,5124 & 2,3083 & 1,9214 & 3,0609 & 3,0867 & 2,2975 & 2,8216 & 27026 & 23334 \\
\hline 2,4936 & 2,2911 & 1,9071 & 3,0379 & 3,0637 & 2,2805 & 2,8004 & 2,6824 & 23161 \\
\hline 2,4749 & 2,2739 & 8929 & 3,0152 & 3,0408 & 2635 & 7794 & 6624 & 2988 \\
\hline 2,4564 & 2,2570 & 1,8788 & 2,9925 & 3,0181 & 2,2467 & 2,7586 & 2,6425 & 2,2817 \\
\hline 2,4379 & 2,2401 & 1,8648 & 29701 & 2,9955 & 2,2300 & 2,7379 & 2,6228 & 2,2648 \\
\hline 2,4197 & 2,2233 & 1,8509 & 2,9478 & 2,9732 & 2,2134 & 2,7174 & 26032 & 2,2479 \\
\hline 2,4015 & 2,2067 & 1,8371 & 2,9257 & 2,9509 & 2,1969 & 2,6971 & 2,5838 & 2,2312 \\
\hline 2,3835 & 2,1902 & 1,8234 & 2,9038 & 2,9289 & 2,1805 & 2,6769 & 2,5645 & 2,2146 \\
\hline 2,3656 & 2,1737 & 1,8098 & 28820 & 2,9070 & 2,1643 & 2,6569 & 454 & 2,1982 \\
\hline 2,3478 & 2,1575 & 1,7963 & 28604 & 2,8853 & 2,1482 & 2,6370 & 2,5265 & 2,1818 \\
\hline 2,3302 & 2,1413 & 1,7828 & 28390 & 2,8637 & 2,1321 & 2,6173 & 2,5076 & 2,1656 \\
\hline 2,3127 & 2,1252 & 1,7695 & 28177 & 2,8423 & 2,1162 & 2,5978 & 2,4890 & 2,1495 \\
\hline 2,2953 & 2,1093 & 1,7563 & 27966 & 2,8211 & 2,1004 & 2,5784 & 2,4704 & 2,1335 \\
\hline 2,2781 & 2,0935 & 1,7431 & 27757 & 2,8000 & 2,0848 & 2,5592 & 2,4520 & 2,1176 \\
\hline 2610 & 20778 & 1,7301 & 27549 & 2,7791 & 2,0692 & 2,5401 & 2,4338 & 2,1018 \\
\hline 2,2440 & 2,0622 & 1,7 & 3 & 2 & 37 & 11 & 56 & 861 \\
\hline 2,2272 & 2,0467 & 1,7042 & 27139 & 2 & 84 & 24 & 976 & 2,0706 \\
\hline 2,2105 & 2,0314 & 1,6915 & 26936 & 2,7172 & 2,0231 & 37 & 23798 & 2,0551 \\
\hline 2,1939 & 20161 & 1,6788 & 2,6735 & 2,6969 & 2,0080 & 2,4652 & 23620 & 2,0398 \\
\hline 2,1775 & 2,0010 & 1,6662 & 26535 & 2,6767 & 1,9930 & 2,4468 & 23444 & 2,0246 \\
\hline 2,1611 & 1,9860 & 1,6537 & 26337 & 2,6567 & 1,9781 & 2,4286 & 233270 & 2,0094 \\
\hline 2,1449 & 1,9711 & 1,6413 & 26140 & 2,6368 & 1,9632 & 2,4105 & 2,3096 & 1,9944 \\
\hline 2,1289 & 1,9563 & 1,6290 & 2,5945 & 2,6171 & 1,9485 & 2,3926 & 2,2924 & 1,9795 \\
\hline 2,1129 & 1,9417 & 1,6168 & 2,5752 & 2,5976 & 1,9340 & 2,3748 & 22753 & 1,9647 \\
\hline 2,0971 & 1,9271 & 1,6046 & 2,5559 & 2,5781 & 1,9195 & 2,3571 & 22583 & 1,9500 \\
\hline 2,0814 & 1,9127 & 1,5926 & 2,5369 & 2,5589 & 1,9051 & 2,3395 & 2,2415 & 1,9354 \\
\hline 2,0658 & 1,8983 & 1,5807 & 2,5180 & 2,5397 & 1,8908 & 2,3221 & 2,2248 & 1,9210 \\
\hline 2,0504 & 1,8841 & 1,5688 & 2,4992 & 2,5207 & 1,8766 & 2,3048 & 22082 & 1,9066 \\
\hline 2,0351 & 1,8700 & 1,5571 & 2,4806 & 2,5019 & 1,8626 & 2,2876 & 2,1917 & 1,8923 \\
\hline 2,0199 & 1,8560 & 1,5454 & 2,4621 & 2,4832 & 1,8486 & 2,2706 & 2,1753 & 1,8781 \\
\hline 2,0048 & 1,8421 & 1,5338 & 24437 & 2,4646 & 1,8348 & 2,2536 & 21590 & 1,8641 \\
\hline 1,9898 & 1,8283 & 1,5223 & 2,4255 & 2,4462 & 1,8210 & 2,2368 & 21429 & 1,8501 \\
\hline 1,9749 & 1,8147 & 1,5109 & 2,4074 & 2,4279 & 1,8074 & 2,2202 & 21269 & 1,8363 \\
\hline 1,9602 & 1,8011 & 1,4996 & 23895 & 2,4098 & 1,7938 & 2,2036 & 21110 & 1,8225 \\
\hline
\end{tabular}


$\begin{array}{llllllllll}\text { Zeit } & \mathrm{P}_{1} & \mathrm{P}_{2} & \mathrm{P}_{3} & \mathrm{P}_{4} & \mathrm{P}_{5} & \mathrm{P}_{6} & \mathrm{P}_{7} & \mathrm{P}_{8} & \mathrm{P}_{9}\end{array}$

$\begin{array}{llllllllll}197,0 & 1,9455 & 1,7876 & 1,4884 & 2,3717 & 2,3918 & 1,7804 & 2,1872 & 2,0952 & 1,8088\end{array}$

$\begin{array}{llllllllll}1972 & 1,9310 & 1,7743 & 1,4772 & 23540 & 2,3739 & 1,7670 & 2,1708 & 2,0795 & 1,7953\end{array}$

$\begin{array}{llllllllll}197,4 & 1,9166 & 1,7610 & 1,4662 & 2,3364 & 2,3561 & 1,7538 & 2,1546 & 2,0640 & 1,7819\end{array}$

$\begin{array}{llllllllll}197,7 & 1,9023 & 1,7479 & 1,4552 & 23190 & 2,3385 & 1,7407 & 2,1385 & 2,0485 & 1,7685\end{array}$

$\begin{array}{llllllllll}1979 & 1,8881 & 1,7348 & 1,4443 & 2,3017 & 2,3211 & 1,7276 & 2,1226 & 20332 & 1,7553\end{array}$

$\begin{array}{llllllllll}\mathbf{1 9 8 , 1} & 1,8740 & 1,7218 & 1,4335 & 22846 & 2,3037 & 1,7147 & 2,1067 & 20180 & 1,7421\end{array}$

$\begin{array}{llllllllll}198,3 & 1,8600 & 1,7090 & 1,4228 & 2,2675 & 2,2865 & 1,7019 & 2,0910 & 2,0029 & 1,7291\end{array}$

$\begin{array}{llllllllll}198,5 & 1,8461 & 1,6962 & 1,4122 & 22506 & 2,2694 & 1,6891 & 2,0754 & 1,9879 & 1,7161\end{array}$

$\begin{array}{llllllllll}198,8 & 1,8324 & 1,6835 & 1,4016 & 2,2338 & 2,2525 & 1,6765 & 2,0599 & 1,9731 & 1,7033\end{array}$

$\begin{array}{llllllllll}199,0 & 1,8187 & 1,6710 & 1,3911 & 22171 & 2,2356 & 1,6640 & 2,0445 & 1,9583 & 1,6905\end{array}$

$\begin{array}{llllllllll}199,2 & 1,8051 & 1,6585 & 1,3808 & 2,2006 & 2,2189 & 1,6515 & 2,0292 & 1,9436 & 1,6779\end{array}$

$\begin{array}{llllllllll}199,4 & 1,7917 & 1,6461 & 1,3704 & 21842 & 2,2023 & 1,6392 & 2,0140 & 1,9291 & 1,6653\end{array}$

$\begin{array}{llllllllll}199,6 & 1,7783 & 1,6338 & 1,3602 & 21679 & 2,1859 & 1,6269 & 1,9990 & 1,9147 & 1,6529\end{array}$

$\begin{array}{llllllllll}199,8 & 1,7650 & 1,6216 & 1,3501 & 21517 & 2,1696 & 1,6148 & 1,9840 & 1,9003 & 1,6405\end{array}$

$\begin{array}{llllllllll}200,1 & 1,7518 & 1,6095 & 1,3400 & 21356 & 2,1534 & 1,6027 & 1,9692 & 1,8861 & 1,6282\end{array}$

$\begin{array}{llllllllll}\mathbf{2 0 0 3} & 1,7388 & 1,5975 & 1,3300 & 21197 & 2,1373 & 1,5907 & 1,9544 & 1,8720 & 1,6160\end{array}$

$\begin{array}{llllllllll}\mathbf{2 0 0 , 5} & 1,7258 & 1,5856 & 1,3200 & 2,1038 & 2,1213 & 1,5788 & 1,9398 & 1,8580 & 1,6040\end{array}$

$\begin{array}{llllllllll}200,7 & 1,7129 & 1,5737 & 1,3102 & 2,0881 & 2,1054 & 1,5670 & 1,9253 & 1,8441 & 1,5920\end{array}$

$\begin{array}{llllllllll}2009 & 1,7001 & 1,5620 & 1,3004 & 2,0725 & 2,0897 & 1,5553 & 1,9109 & 1,8303 & 1,5801\end{array}$

$\begin{array}{llllllllll}\mathbf{2 0 1 2} & 1,6874 & 1,5503 & 1,2907 & 20570 & 2,0741 & 1,5437 & 1,8966 & 1,8166 & 1,5683\end{array}$

$\begin{array}{llllllllll}\mathbf{2 0 1 , 4} & 1,6748 & 1,5387 & 1,2811 & 2,0417 & 2,0586 & 1,5322 & 1,8825 & 1,8030 & 1,5566\end{array}$

$\begin{array}{llllllllll}201,6 & 1,6623 & 1,5273 & 1,2715 & 2,0264 & 2,0432 & 1,5208 & 1,8684 & 1,7896 & 1,5449\end{array}$

$\begin{array}{llllllllll}\mathbf{2 0 1 , 8} & 1,6498 & 1,5158 & 1,2620 & 2,0113 & 2,0280 & 1,5094 & 1,8544 & 1,7762 & 1,5334\end{array}$

$\begin{array}{llllllllll}\mathbf{2 0 1 , 8} & 1,6474 & 1,5136 & 1,2601 & 2,0082 & 2,0249 & 1,5071 & 1,8516 & 1,7735 & 1,5311\end{array}$

$\begin{array}{llllllllll}202,1 & 1,6363 & 1,5034 & 1,2516 & 1,9947 & 2,0113 & 1,4970 & 1,8391 & 1,7616 & 1,5208\end{array}$

$\begin{array}{llllllllll}\mathbf{2 0 2 , 3} & 1,6241 & 1,4922 & 1,2423 & 1,9798 & 1,9963 & 1,4858 & 1,8254 & 1,7484 & 1,5094\end{array}$

$\begin{array}{llllllllll}\mathbf{2 0 2 5} & 1,6119 & 1,4810 & 1,2330 & 1,9650 & 1,9814 & 1,4747 & 1,8117 & 1,7353 & 1,4982\end{array}$

$\begin{array}{llllllllll}202,7 & 1,5999 & 1,4699 & 1,2238 & 1,9503 & 1,9666 & 1,4637 & 1,7982 & 1,7224 & 1,4870\end{array}$

$\begin{array}{llllllllll}202,9 & 1,5879 & 1,4590 & 1,2147 & 1,9357 & 1,9519 & 1,4528 & 1,7848 & 1,7095 & 1,4759\end{array}$

$\begin{array}{llllllllll}\mathbf{2 0 3 2} & 1,5760 & 1,4480 & 1,2056 & 1,9213 & 1,9373 & 1,4419 & 1,7714 & 1,6967 & 1,4649\end{array}$

$\begin{array}{llllllllll}\mathbf{2 0 3 , 4} & 1,5642 & 1,4372 & 1,1966 & 1,9069 & 1,9228 & 1,4312 & 1,7582 & 1,6841 & 1,4539\end{array}$

$\begin{array}{llllllllll}201,6 & 1,5525 & 1,4265 & 1,1877 & 1,8927 & 1,9084 & 1,4205 & 1,7450 & 1,6715 & 1,4431\end{array}$

$\begin{array}{llllllllll}\mathbf{2 0 3 , 8} & 1,5409 & 1,4158 & 1,1788 & 1,8785 & 1,8942 & 1,4099 & 1,7320 & 1,6590 & 1,4323\end{array}$

$\begin{array}{llllllllll}\mathbf{2 0 4 , 0} & 1,5294 & 1,4052 & 1,1700 & 1,8645 & 1,8800 & 1,3993 & 1,7191 & 1,6466 & 1,4216\end{array}$

$\begin{array}{llllllllll}\mathbf{2 0 4 , 2} & 1,5180 & 1,3947 & 1,1612 & 1,8505 & 1,8660 & 1,3889 & 1,7062 & 1,6343 & 1,4110\end{array}$

$\begin{array}{llllllllll}\mathbf{2 0 4 , 5} & 1,5066 & 1,3843 & 1,1526 & 1,8367 & 1,8520 & 1,3785 & 1,6935 & 1,6221 & 1,4005\end{array}$

$\begin{array}{llllllllll}\mathbf{2 0 4 7} & 1,4953 & 1,3739 & 1,1439 & 1,8230 & 1,8382 & 1,3682 & 1,6808 & 1,6100 & 1,3900\end{array}$

$\begin{array}{llllllllll}\mathbf{2 0 4 9} 9 & 1,4841 & 1,3637 & 1,1354 & 1,8094 & 1,8245 & 1,3580 & 1,6683 & 1,5980 & 1,3796\end{array}$ 
$\begin{array}{llllllllll}\text { Zeit } & \mathrm{P}_{1} & \mathrm{P}_{2} & \mathrm{P}_{3} & \mathrm{P}_{4} & \mathrm{P}_{5} & \mathrm{P}_{6} & \mathrm{P}_{7} & \mathrm{P}_{8} & \mathrm{P}_{9}\end{array}$

$\begin{array}{llllllllll}205,1 & 1,4730 & 1,3535 & 1,1269 & 1,7958 & 1,8108 & 1,3479 & 1,6558 & 1,5860 & 1,3693\end{array}$

$\begin{array}{lllllllllll}2053 & 1,4620 & 1,3433 & 1,1185 & 1,7824 & 1,7973 & 1,3378 & 1,6434 & 1,5742 & 1,3591\end{array}$

$\begin{array}{llllllllll}205,6 & 1,4511 & 1,3333 & 1,1101 & 1,7691 & 1,7839 & 1,3278 & 1,6312 & 1,5624 & 1,3489\end{array}$

$\begin{array}{llllllllll}205,8 & 1,4402 & 1,3233 & 1,1018 & 1,7559 & 1,7705 & 1,3179 & 1,6190 & 1,5508 & 1,3389\end{array}$

$\begin{array}{llllllllll}206,0 & 1,4295 & 1,3134 & 1,0936 & 1,7428 & 1,7573 & 1,3080 & 1,6069 & 1,5392 & 1,3289\end{array}$

$\begin{array}{llllllllll}2066,2 & 1,4188 & 1,3036 & 1,0854 & 1,7298 & 1,7442 & 1,2982 & 1,5949 & 1,527 & 1,3189\end{array}$

$\begin{array}{llllllllll}206,4 & 1,4082 & 1,2938 & 1,0773 & 1,7168 & 1,7311 & 1,2885 & 1,5830 & 1,5163 & 1,3091\end{array}$

$\begin{array}{lllllllllll}2006,6 & 1,3976 & 1,2842 & 1,0692 & 1,7040 & 1,7182 & 1,2789 & 1,5712 & 1,5050 & 1,2993\end{array}$

$\begin{array}{llllllllll}2066,9 & 1,3872 & 1,2746 & 1,0612 & 1,6913 & 1,7054 & 1,2693 & 1,5594 & 1,4937 & 1,2896\end{array}$

$\begin{array}{llllllllll}\mathbf{2 0 7 , 1} & 1,3768 & 1,2650 & 1,0533 & 1,6786 & 1,6926 & 1,2598 & 1,5478 & 1,4826 & 1,2800\end{array}$

$\begin{array}{llllllllll}2073 & 1,3665 & 1,2556 & 1,0454 & 1,6661 & 1,6800 & 1,2504 & 1,5362 & 1,4715 & 1,2704\end{array}$

$\begin{array}{llllllllll}\mathbf{2 0 7 5} & 1,3563 & 1,2462 & 1,0376 & 1,6537 & 1,6674 & 1,2411 & 1,5248 & 1,4605 & 1,2609\end{array}$

$\begin{array}{llllllllll}207,7 & 1,3461 & 1,2368 & 1,0298 & 1,6413 & 1,6549 & 1,2318 & 1,5134 & 1,4496 & 1,2515\end{array}$

$\begin{array}{llllllllll}208,0 & 1,3361 & 1,2276 & 1,0221 & 1,6291 & 1,6426 & 1,2226 & 1,5021 & 1,4388 & 1,2421\end{array}$

$\begin{array}{llllllllll}\mathbf{2 0 8 , 2} & 1,3261 & 1,2184 & 1,0145 & 1,6169 & 1,6303 & 1,2134 & 1,4909 & 1,4280 & 1,2328\end{array}$

$\begin{array}{llllllllll}208,4 & 1,3162 & 1,2093 & 1,0069 & 1,6048 & 1,6181 & 1,2044 & 1,4797 & 1,4174 & 1,2236\end{array}$

$\begin{array}{llllllllll}\mathbf{2 0 8 , 6} & 1,3063 & 1,2003 & 0,9994 & 1,5929 & 1,6060 & 1,1954 & 1,4687 & 1,4068 & 1,2145\end{array}$

$\begin{array}{llllllllll}\mathbf{2 0 8 , 8} & 1,2966 & 1,1913 & 0,9919 & 1,5810 & 1,5940 & 1,1864 & 1,4577 & 1,3963 & 1,2054\end{array}$

$\begin{array}{llllllllll}209,0 & 1,2869 & 1,1824 & 0,9845 & 1,5692 & 1,5821 & 1,1775 & 1,4468 & 1,3858 & 1,1964\end{array}$

$\begin{array}{llllllllll}2093 & 1,2773 & 1,1735 & 0,9771 & 1,5574 & 1,5703 & 1,1687 & 1,4360 & 1,3755 & 1,1874\end{array}$

$\begin{array}{llllllllll}209,5 & 1,2677 & 1,1648 & 0,9698 & 1,5458 & 1,5586 & 1,1600 & 1,4253 & 1,3652 & 1,1786\end{array}$

$\begin{array}{llllllllll}209,7 & 1,2582 & 1,1561 & 0,9626 & 1,5343 & 1,5469 & 1,1513 & 1,4147 & 1,3550 & 1,1697\end{array}$

$\begin{array}{llllllllll}209,9 & 1,2488 & 1,1474 & 0,9554 & 1,5228 & 1,5354 & 1,1427 & 1,4041 & 1,3449 & 1,1610\end{array}$

$\begin{array}{llllllllll}210,1 & 1,2395 & 1,1388 & 0,9482 & 1,5114 & 1,5239 & 1,1342 & 1,3936 & 1,3348 & 1,1523\end{array}$

$\begin{array}{llllllllll}210,4 & 1,2303 & 1,1303 & 0,9411 & 1,5002 & 1,5125 & 1,1257 & 1,3832 & 1,3248 & 1,1437\end{array}$

$\begin{array}{llllllllll}210,6 & 1,2211 & 1,1219 & 0,9341 & 1,4890 & 1,5012 & 1,1173 & 1,3729 & 1,3149 & 1,1352\end{array}$

$\begin{array}{llllllllll}210,8 & 1,2119 & 1,1135 & 0,9271 & 1,4778 & 1,4900 & 1,1089 & 1,3626 & 1,3051 & 1,1267\end{array}$

$\begin{array}{llllllllll}211,0 & 1,2029 & 1,1052 & 0,9202 & 1,4668 & 1,4788 & 1,1006 & 1,3524 & 1,2954 & 1,1182\end{array}$

$\begin{array}{llllllllll}211,2 & 1,1939 & 1,0969 & 0,9133 & 1,4559 & 1,4678 & 1,0924 & 1,3423 & 1,2857 & 1,1099\end{array}$

$\begin{array}{llllllllll}\mathbf{2 1 1 , 4} & 1,1850 & 1,0887 & 0,9065 & 1,4450 & 1,4568 & 1,0842 & 1,3323 & 1,2761 & 1,1016\end{array}$

$\begin{array}{llllllllll}211,7 & 1,1761 & 1,0806 & 0,8997 & 1,4342 & 1,4459 & 1,0761 & 1,3224 & 1,2665 & 1,0934\end{array}$

$\begin{array}{llllllllll}211,9 & 1,1673 & 1,0725 & 0,8930 & 1,4235 & 1,4351 & 1,0681 & 1,3125 & 1,2571 & 1,0852\end{array}$

$\begin{array}{llllllllll}212,1 & 1,1586 & 1,0645 & 0,8863 & 1,4129 & 1,4244 & 1,0601 & 1,3027 & 1,2477 & 1,0771\end{array}$

$\begin{array}{llllllllll}212,3 & 1,1500 & 1,0565 & 0,8797 & 1,4023 & 1,4138 & 1,0522 & 1,2929 & 1,2383 & 1,0690\end{array}$

$\begin{array}{llllllllll}212,5 & 1,1414 & 1,0487 & 0,8731 & 1,3918 & 1,4032 & 1,0443 & 1,2833 & 1,2291 & 1,0610\end{array}$

$\begin{array}{llllllllll}212,8 & 1,1328 & 1,0408 & 0,8666 & 1,3814 & 1,3927 & 1,0365 & 1,2737 & 1,2199 & 1,0531\end{array}$

$\begin{array}{llllllllll}\mathbf{2 1 3 , 0} & 1,1244 & 1,0330 & 0,8601 & 1,3711 & 1,3823 & 1,0288 & 1,2642 & 1,2108 & 1,0452\end{array}$

$\begin{array}{llllllllll}\mathbf{2 1 3 , 2} & 1,1160 & 1,0253 & 0,8537 & 1,3609 & 1,3720 & 1,0211 & 1,2547 & 1,2017 & 1,0374\end{array}$ 
$\begin{array}{llllllllll}\text { Zeit } & \mathrm{P}_{1} & \mathrm{P}_{2} & \mathrm{P}_{3} & \mathrm{P}_{4} & \mathrm{P}_{5} & \mathrm{P}_{6} & \mathrm{P}_{7} & \mathrm{P}_{8} & \mathrm{P}_{9}\end{array}$

$\begin{array}{llllllllll}213,4 & 1,1076 & 1,0177 & 0,8473 & 1,3507 & 1,3617 & 1,0134 & 1,2454 & 1,1928 & 1,0297\end{array}$

$\begin{array}{llllllllll}\mathbf{2 1 3 , 6} & 1,0994 & 1,0101 & 0,8410 & 1,3406 & 1,3516 & 1,0059 & 1,2361 & 1,1838 & 1,0220\end{array}$

$\begin{array}{llllllllll}213,8 & 1,0911 & 1,0025 & 0,8347 & 1,3306 & 1,3415 & 0,9984 & 1,2268 & 1,1750 & 1,0143\end{array}$

$\begin{array}{llllllllll}214,1 & 1,0830 & 0,9950 & 0,8285 & 1,3207 & 1,3314 & 0,9909 & 1,2177 & 1,1662 & 1,0067\end{array}$

$\begin{array}{llllllllll}\mathbf{2 1 4 3} & 1,0749 & 0,9876 & 0,8223 & 1,3108 & 1,3215 & 0,9835 & 1,2086 & 1,1575 & 0,9992\end{array}$

$\begin{array}{llllllllll}\mathbf{2 1 4 , 5} & 1,0669 & 0,9802 & 0,8161 & 1,3010 & 1,3116 & 0,9761 & 1,1995 & 1,1488 & 0,9918\end{array}$

$\begin{array}{llllllllll}\mathbf{2 1 4 , 7} & 1,0589 & 0,9729 & 0,8100 & 1,2913 & 1,3018 & 0,9688 & 1,1906 & 1,1403 & 0,9843\end{array}$

$\begin{array}{llllllllll}\mathbf{2 1 4 9} & 1,0510 & 0,9656 & 0,8040 & 1,2816 & 1,2921 & 0,9616 & 1,1817 & 1,1317 & 0,970\end{array}$

$\begin{array}{llllllllll}215,1 & 1,0431 & 0,9584 & 0,7980 & 1,2721 & 1,2824 & 0,9544 & 1,1728 & 1,1233 & 0,9697\end{array}$

$\begin{array}{llllllllll}\mathbf{2 1 5 , 4} & 1,0353 & 0,9512 & 0,7920 & 1,2626 & 1,2729 & 0,4473 & 1,1641 & 1,1149 & 0,9624\end{array}$

$\begin{array}{llllllllll}215,6 & 1,0276 & 0,9441 & 0,7861 & 1,2531 & 1,2633 & 0,9402 & 1,1554 & 1,1066 & 0,9553\end{array}$

$\begin{array}{llllllllll}\mathbf{2 1 5 , 8} & 1,0199 & 0,9371 & 0,7802 & 1,2438 & 1,2539 & 0,9332 & 1,1467 & 1,0983 & 0,9481\end{array}$

$\begin{array}{llllllllll}\mathbf{2 1 6 , 0} & 1,0123 & 0,9301 & 0,7744 & 1,2345 & 1,2445 & 0,9262 & 1,1382 & 1,0901 & 0,9410\end{array}$

$\begin{array}{llllllllll}\mathbf{2 1 6 , 2} & 1,0047 & 0,9231 & 0,7686 & 1,2253 & 1,2352 & 0,9193 & 1,1297 & 1,0819 & 0,9340\end{array}$

$\begin{array}{llllllllll}216,5 & 0,9972 & 0,9162 & 0,7628 & 1,2161 & 1,2260 & 0,9124 & 1,1212 & 1,0739 & 0,9270\end{array}$

$\begin{array}{llllllllll}216,7 & 0,9898 & 0,9094 & 0,7571 & 1,2070 & 1,2168 & 0,9056 & 1,1129 & 1,0658 & 0,9201\end{array}$

$\begin{array}{llllllllll}216,9 & 0,9824 & 0,9026 & 0,7515 & 1,1980 & 1,2078 & 0,8988 & 1,1045 & 1,0579 & 0,9132\end{array}$

$\begin{array}{llllllllll}217,1 & 0,9750 & 0,8958 & 0,7459 & 1,1890 & 1,1987 & 0,8921 & 1,0963 & 1,0500 & 0,9064\end{array}$

$\begin{array}{llllllllll}2173 & 0,9677 & 0,8891 & 0,7403 & 1,1802 & 1,1898 & 0,8855 & 1,0881 & 1,0421 & 0,8996\end{array}$

$\begin{array}{llllllllll}217,5 & 0,9605 & 0,8825 & 0,7348 & 1,1714 & 1,1809 & 0,8788 & 1,0800 & 1,0343 & 0,8929\end{array}$

$\begin{array}{llllllllll}\mathbf{2 1 7 , 8} & 0,9533 & 0,8759 & 0,7293 & 1,1626 & 1,1721 & 0,8723 & 1,0719 & 1,0266 & 0,8862\end{array}$

$\begin{array}{llllllllll}\mathbf{2 1 8 , 0} & 0,9462 & 0,8693 & 0,7238 & 1,1539 & 1,1633 & 0,8658 & 1,0639 & 1,0189 & 0,8796\end{array}$

$\begin{array}{llllllllll}\mathbf{2 1 8 , 2} & 0,9391 & 0,8628 & 0,7184 & 1,1453 & 1,1546 & 0,8593 & 1,0559 & 1,0113 & 0,8730\end{array}$

$\begin{array}{llllllllll}218,4 & 0,9321 & 0,8564 & 0,7130 & 1,1367 & 1,1460 & 0,8529 & 1,0481 & 1,0038 & 0,8665\end{array}$

$\begin{array}{llllllllll}218,6 & 0,9251 & 0,8500 & 0,7077 & 1,1282 & 1,1374 & 0,8465 & 1,0402 & 0,9963 & 0,8600\end{array}$

$\begin{array}{llllllllll}\mathbf{2 1 8 , 9} & 0,9182 & 0,8436 & 0,7024 & 1,1198 & 1,1289 & 0,8402 & 1,0325 & 0,9888 & 0,8536\end{array}$

$\begin{array}{llllllllll}219,1 & 0,9114 & 0,8373 & 0,6972 & 1,1115 & 1,1205 & 0,8339 & 1,0248 & 0,9814 & 0,8472\end{array}$

$\begin{array}{llllllllll}2193 & 0,9045 & 0,8311 & 0,6920 & 1,1032 & 1,1121 & 0,827 & 1,0171 & 0,9741 & 0,8409\end{array}$

$\begin{array}{llllllllll}\mathbf{2 1 9 , 5} & 0,8978 & 0,8249 & 0,6868 & 1,0949 & 1,1038 & 0,8215 & 1,0095 & 0,9668 & 0,8346\end{array}$

$\begin{array}{llllllllll}\mathbf{2 1 9 , 7} & 0,8911 & 0,8187 & 0,6817 & 1,0867 & 1,0956 & 0,8153 & 1,0020 & 0,9596 & 0,8284\end{array}$

$\begin{array}{llllllllll}219,9 & 0,8844 & 0,8126 & 0,6766 & 1,0786 & 1,0874 & 0,8092 & 0,9945 & 0,9524 & 0,8222\end{array}$

$\begin{array}{llllllllll}220,2 & 0,8778 & 0,8065 & 0,6715 & 1,0706 & 1,0792 & 0,8032 & 0,9870 & 0,9453 & 0,8161\end{array}$

$\begin{array}{llllllllll}220,4 & 0,8712 & 0,8005 & 0,6665 & 1,0626 & 1,0712 & 0,7972 & 0,9797 & 0,9383 & 0,8100\end{array}$

$\begin{array}{llllllllll}220,6 & 0,8647 & 0,7945 & 0,6615 & 1,0546 & 1,0632 & 0,7912 & 0,9724 & 0,9313 & 0,8039\end{array}$

$\begin{array}{llllllllll}220,8 & 0,8583 & 0,7885 & 0,6566 & 1,0467 & 1,0552 & 0,7853 & 0,9651 & 0,9243 & 0,7979\end{array}$

$\begin{array}{llllllllll}221,0 & 0,8519 & 0,7826 & 0,6517 & 1,0389 & 1,0473 & 0,7794 & 0,9579 & 0,9174 & 0,7919\end{array}$

$\begin{array}{llllllllll}221,3 & 0,8455 & 0,7768 & 0,6468 & 1,0312 & 1,0395 & 0,736 & 0,9507 & 0,9105 & 0,7860\end{array}$

$\begin{array}{llllllllll}221,5 & 0,8392 & 0,7710 & 0,6420 & 1,0235 & 1,0318 & 0,7678 & 0,4436 & 0,9037 & 0,7801\end{array}$ 
$\begin{array}{llllllllll}\text { Zeit } & \mathrm{P}_{1} & \mathrm{P}_{2} & \mathrm{P}_{3} & \mathrm{P}_{4} & \mathrm{P}_{5} & \mathrm{P}_{6} & \mathrm{P}_{7} & \mathrm{P}_{8} & \mathrm{P}_{9}\end{array}$

$\begin{array}{llllllllll}221,7 & 0,8329 & 0,7652 & 0,6372 & 1,0158 & 1,0240 & 0,7621 & 0,9366 & 0,8970 & 0,7743\end{array}$

$\begin{array}{llllllllll}221,9 & 0,8267 & 0,7595 & 0,6324 & 1,0082 & 1,0164 & 0,7564 & 0,9296 & 0,8903 & 0,7685\end{array}$

$\begin{array}{llllllllll}222,1 & 0,8205 & 0,7538 & 0,6277 & 1,0007 & 1,0088 & 0,7507 & 0,9226 & 0,8836 & 0,7628\end{array}$

$\begin{array}{llllllllll}222,3 & 0,8144 & 0,7482 & 0,6230 & 0,9932 & 1,0013 & 0,7451 & 0,9157 & 0,8770 & 0,7571\end{array}$

$\begin{array}{llllllllll}222,6 & 0,8083 & 0,7426 & 0,6183 & 0,9858 & 0,9938 & 0,7396 & 0,9089 & 0,8705 & 0,7514\end{array}$

$\begin{array}{llllllllll}222,8 & 0,8022 & 0,7371 & 0,6137 & 0,9784 & 0,9864 & 0,7340 & 0,9021 & 0,8640 & 0,7458\end{array}$

$\begin{array}{llllllllll}223,0 & 0,7962 & 0,7316 & 0,6091 & 0,9711 & 0,9790 & 0,7286 & 0,8954 & 0,8575 & 0,7402\end{array}$

$\begin{array}{llllllllll}223,2 & 0,7903 & 0,7261 & 0,6046 & 0,6639 & 0,9717 & 0,7231 & 0,8887 & 0,8511 & 0,7347\end{array}$

$\begin{array}{llllllllll}223,4 & 0,7844 & 0,7207 & 0,6000 & 0,9567 & 0,9644 & 0,7177 & 0,8820 & 0,8447 & 0,7222\end{array}$

$\begin{array}{llllllllll}223,7 & 0,7785 & 0,7153 & 0,5956 & 0,4495 & 0,9572 & 0,7123 & 0,8755 & 0,8384 & 0,7238\end{array}$

$\begin{array}{llllllllll}223,9 & 0,7727 & 0,7099 & 0,5911 & 0,4424 & 0,9501 & 0,7070 & 0,8689 & 0,8322 & 0,7184\end{array}$

$\begin{array}{llllllllll}224,1 & 0,7669 & 0,7046 & 0,5867 & 0,9354 & 0,9430 & 0,7017 & 0,8624 & 0,8260 & 0,7130\end{array}$

$\begin{array}{llllllllll}2243 & 0,7612 & 0,6994 & 0,5823 & 0,9284 & 0,9359 & 0,6965 & 0,8560 & 0,8198 & 0,7077\end{array}$

$\begin{array}{llllllllll}224,5 & 0,7555 & 0,6941 & 0,5780 & 0,9215 & 0,9289 & 0,6913 & 0,8496 & 0,8137 & 0,7024\end{array}$

$\begin{array}{llllllllll}224,7 & 0,7499 & 0,6889 & 0,5736 & 0,9146 & 0,9220 & 0,6861 & 0,8432 & 0,8076 & 0,6971\end{array}$

$\begin{array}{llllllllll}\mathbf{2 2 5 , 0} & 0,7443 & 0,6838 & 0,5693 & 0,9078 & 0,9151 & 0,6810 & 0,8369 & 0,8015 & 0,6919\end{array}$

$\begin{array}{llllllllll}225,2 & 0,7387 & 0,6787 & 0,5651 & 0,9010 & 0,9083 & 0,6759 & 0,8307 & 0,7956 & 0,6867\end{array}$

$\begin{array}{llllllllll}225,4 & 0,7332 & 0,6736 & 0,5609 & 0,8943 & 0,9015 & 0,6709 & 0,8245 & 0,7896 & 0,6816\end{array}$

$\begin{array}{llllllllll}225,6 & 0,727 & 0,6686 & 0,5567 & 0,8876 & 0,8947 & 0,6658 & 0,8183 & 0,7837 & 0,6765\end{array}$

$\begin{array}{llllllllll}\mathbf{2 2 5 , 8} & 0,7223 & 0,6636 & 0,5525 & 0,8809 & 0,8880 & 0,6609 & 0,8122 & 0,777 & 0,6715\end{array}$

$\begin{array}{llllllllll}226,1 & 0,7169 & 0,6586 & 0,3484 & 0,8744 & 0,8814 & 0,6559 & 0,8061 & 0,7720 & 0,6664\end{array}$

$\begin{array}{llllllllll}2263 & 0,7115 & 0,6537 & 0,5443 & 0,8678 & 0,8748 & 0,6510 & 0,8001 & 0,7663 & 0,6615\end{array}$

$\begin{array}{llllllllll}226,5 & 0,7062 & 0,6488 & 0,5402 & 0,8614 & 0,8683 & 0,6462 & 0,7941 & 0,7605 & 0,6565\end{array}$

$\begin{array}{llllllllll}226,7 & 0,7009 & 0,6440 & 0,5362 & 0,8549 & 0,8618 & 0,6413 & 0,7882 & 0,7549 & 0,6516\end{array}$

$\begin{array}{llllllllll}226,9 & 0,6957 & 0,6392 & 0,5322 & 0,8485 & 0,8554 & 0,6365 & 0,782 & 0,7492 & 0,6468\end{array}$

$\begin{array}{llllllllll}227,1 & 0,6905 & 0,6344 & 0,5282 & 0,8422 & 0,8490 & 0,6318 & 0,7765 & 0,7436 & 0,6419\end{array}$

$\begin{array}{llllllllll}227,4 & 0,6853 & 0,6296 & 0,5243 & 0,8359 & 0,8426 & 0,6271 & 0,7707 & 0,7381 & 0,6371\end{array}$

$\begin{array}{llllllllll}\mathbf{2 2 7 , 6} & 0,6802 & 0,6249 & 0,5203 & 0,8297 & 0,8363 & 0,6224 & 0,7649 & 0,7326 & 0,6324\end{array}$

$\begin{array}{llllllllll}\mathbf{2 2 7 , 8} & 0,6751 & 0,6203 & 0,5165 & 0,8235 & 0,8301 & 0,617 & 0,7592 & 0,7271 & 0,6276\end{array}$

$\begin{array}{llllllllll}228,0 & 0,6701 & 0,6156 & 0,5126 & 0,8173 & 0,8239 & 0,6131 & 0,7535 & 0,7217 & 0,6229\end{array}$

$\begin{array}{llllllllll}228,2 & 0,6651 & 0,6110 & 0,5088 & 0,8112 & 0,8177 & 0,6085 & 0,7479 & 0,7163 & 0,6183\end{array}$

$\begin{array}{llllllllll}228,5 & 0,6601 & 0,6065 & 0,5050 & 0,8051 & 0,8116 & 0,6040 & 0,7423 & 0,7109 & 0,6137\end{array}$

$\begin{array}{llllllllll}228,7 & 0,6552 & 0,6019 & 0,5012 & 0,7991 & 0,8055 & 0,5995 & 0,7368 & 0,7056 & 0,6091\end{array}$

$\begin{array}{llllllllll}228,9 & 0,6503 & 0,5974 & 0,4974 & 0,7932 & 0,7995 & 0,5950 & 0,7313 & 0,7003 & 0,6045\end{array}$

$\begin{array}{llllllllll}229,1 & 0,6454 & 0,5930 & 0,4937 & 0,7872 & 0,7936 & 0,5905 & 0,7258 & 0,6951 & 0,6000\end{array}$

$\begin{array}{llllllllll}229,3 & 0,6406 & 0,5885 & 0,4900 & 0,7814 & 0,7876 & 0,5861 & 0,7204 & 0,6899 & 0,5955\end{array}$

$\begin{array}{llllllllll}229,5 & 0,6358 & 0,5841 & 0,4864 & 0,7755 & 0,7817 & 0,5817 & 0,7150 & 0,6847 & 0,5911\end{array}$

$\begin{array}{llllllllll}229,8 & 0,6311 & 0,5798 & 0,4827 & 0,7697 & 0,7759 & 0,5774 & 0,7097 & 0,6796 & 0,5867\end{array}$ 
$\begin{array}{llllllllll}\text { Zeit } & \mathrm{P}_{1} & \mathrm{P}_{2} & \mathrm{P}_{3} & \mathrm{P}_{4} & \mathrm{P}_{5} & \mathrm{P}_{6} & \mathrm{P}_{7} & \mathrm{P}_{8} & \mathrm{P}_{9}\end{array}$

$\begin{array}{llllllllll}230,0 & 0,6263 & 0,5754 & 0,4791 & 0,7640 & 0,7701 & 0,5731 & 0,7044 & 0,6746 & 0,5823\end{array}$

$\begin{array}{llllllllll}230,2 & 0,6217 & 0,5711 & 0,4756 & 0,7583 & 0,7644 & 0,5688 & 0,6991 & 0,6695 & 0,5779\end{array}$

$\begin{array}{llllllllll}230,4 & 0,6170 & 0,5669 & 0,4720 & 0,7526 & 0,7586 & 0,5646 & 0,6939 & 0,6645 & 0,5736\end{array}$

$\begin{array}{llllllllll}230,6 & 0,6124 & 0,5626 & 0,4685 & 0,7470 & 0,7530 & 0,5603 & 0,6887 & 0,6595 & 0,5693\end{array}$

$\begin{array}{llllllllll}230,9 & 0,6078 & 0,5584 & 0,4650 & 0,7414 & 0,7473 & 0,5561 & 0,6835 & 0,6546 & 0,5651\end{array}$

$\begin{array}{llllllllll}231,1 & 0,6033 & 0,5543 & 0,4615 & 0,7359 & 0,7418 & 0,5520 & 0,6784 & 0,6497 & 0,5609\end{array}$

$\begin{array}{llllllllll}231,3 & 0,5988 & 0,5501 & 0,4581 & 0,7304 & 0,7362 & 0,5479 & 0,6734 & 0,6449 & 0,5567\end{array}$

$\begin{array}{llllllllll}231,5 & 0,5943 & 0,5460 & 0,4546 & 0,7249 & 0,7307 & 0,5438 & 0,6683 & 0,6401 & 0,5525\end{array}$

$\begin{array}{llllllllll}231,7 & 0,5899 & 0,5419 & 0,4512 & 0,7195 & 0,7253 & 0,5397 & 0,6633 & 0,6353 & 0,5484\end{array}$

$\begin{array}{llllllllll}231,9 & 0,5855 & 0,5379 & 0,4479 & 0,7141 & 0,7198 & 0,5357 & 0,6584 & 0,6305 & 0,5443\end{array}$

$\begin{array}{llllllllll}232,2 & 0,5811 & 0,5339 & 0,4445 & 0,7088 & 0,7145 & 0,5317 & 0,6535 & 0,6258 & 0,5402\end{array}$

$\begin{array}{llllllllll}232,4 & 0,5767 & 0,5299 & 0,4112 & 0,7035 & 0,7091 & 0,527 & 0,6486 & 0,6211 & 0,5362\end{array}$

$\begin{array}{llllllllll}232,6 & 0,5724 & 0,5259 & 0,4379 & 0,6982 & 0,7038 & 0,5238 & 0,6437 & 0,6165 & 0,5322\end{array}$

$\begin{array}{llllllllll}232,8 & 0,5682 & 0,5220 & 0,4346 & 0,6930 & 0,6986 & 0,5198 & 0,6389 & 0,6119 & 0,5282\end{array}$

$\begin{array}{llllllllll}\mathbf{2 3 3 , 0} & 0,5639 & 0,5181 & 0,4314 & 0,6878 & 0,6933 & 0,5160 & 0,6342 & 0,6073 & 0,5242\end{array}$

$\begin{array}{llllllllll}233,2 & 0,5597 & 0,5142 & 0,4282 & 0,6827 & 0,6882 & 0,5121 & 0,6294 & 0,6028 & 0,5203\end{array}$

$\begin{array}{llllllllll}233,5 & 0,5555 & 0,5104 & 0,4250 & 0,6776 & 0,6830 & 0,5083 & 0,6247 & 0,5983 & 0,5164\end{array}$

$\begin{array}{llllllllll}233,7 & 0,5514 & 0,5066 & 0,4218 & 0,6725 & 0,6779 & 0,5045 & 0,6200 & 0,5938 & 0,5126\end{array}$

$\begin{array}{llllllllll}2333,9 & 0,5472 & 0,5028 & 0,4186 & 0,6675 & 0,6729 & 0,5007 & 0,6154 & 0,5894 & 0,5088\end{array}$

$\begin{array}{llllllllll}234,1 & 0,5432 & 0,4990 & 0,4155 & 0,6625 & 0,6678 & 0,4970 & 0,6108 & 0,5850 & 0,5050\end{array}$

$\begin{array}{llllllllll}\mathbf{2 3 4}, 3 & 0,5391 & 0,4953 & 0,4124 & 0,6576 & 0,6628 & 0,4933 & 0,6063 & 0,5806 & 0,5012\end{array}$

$\begin{array}{llllllllll}234,6 & 0,5351 & 0,4916 & 0,4093 & 0,6527 & 0,6579 & 0,4896 & 0,6017 & 0,5763 & 0,4974\end{array}$

$\begin{array}{llllllllll}\mathbf{2 3 4 , 8} & 0,5311 & 0,4879 & 0,4063 & 0,6478 & 0,6530 & 0,4859 & 0,5972 & 0,5720 & 0,4937\end{array}$

$\begin{array}{llllllllll}\mathbf{2 3 5 , 0} & 0,5271 & 0,4843 & 0,4032 & 0,6429 & 0,6481 & 0,4823 & 0,5928 & 0,5677 & 0,4900\end{array}$

$\begin{array}{llllllllll}235,2 & 0,5232 & 0,4806 & 0,4002 & 0,6381 & 0,6433 & 0,4787 & 0,5883 & 0,5634 & 0,4864\end{array}$

$\begin{array}{llllllllll}\mathbf{2 3 5 , 4} & 0,5193 & 0,4771 & 0,3972 & 0,6334 & 0,6384 & 0,4751 & 0,5839 & 0,5592 & 0,4827\end{array}$

$\begin{array}{llllllllll}235,6 & 0,5154 & 0,4735 & 0,3942 & 0,6286 & 0,6337 & 0,4716 & 0,5796 & 0,5551 & 0,4791\end{array}$

$\begin{array}{llllllllll}\mathbf{2 3 5}, 9 & 0,5115 & 0,4700 & 0,3913 & 0,6240 & 0,6289 & 0,4680 & 0,5753 & 0,5509 & 0,4755\end{array}$

$\begin{array}{llllllllll}236,1 & 0,507 & 0,4664 & 0,3884 & 0,6193 & 0,6242 & 0,4645 & 0,5710 & 0,5468 & 0,4720\end{array}$

$\begin{array}{llllllllll}236,3 & 0,5039 & 0,4630 & 0,3855 & 0,6147 & 0,6196 & 0,4611 & 0,5667 & 0,5427 & 0,4685\end{array}$

$\begin{array}{llllllllll}236,5 & 0,5001 & 0,4595 & 0,3826 & 0,6101 & 0,6149 & 0,4576 & 0,5625 & 0,5386 & 0,4650\end{array}$

$\begin{array}{llllllllll}\mathbf{2 3 6 , 7} & 0,4964 & 0,4561 & 0,3797 & 0,6055 & 0,6104 & 0,4542 & 0,5583 & 0,5346 & 0,4615\end{array}$

$\begin{array}{llllllllll}237,0 & 0,4927 & 0,4527 & 0,3769 & 0,6010 & 0,6058 & 0,4508 & 0,5541 & 0,5306 & 0,4580\end{array}$

$\begin{array}{llllllllll}237,2 & 0,4890 & 0,4493 & 0,3741 & 0,5965 & 0,6013 & 0,4474 & 0,5499 & 0,5267 & 0,4546\end{array}$

$\begin{array}{llllllllll}\mathbf{2 3 7 , 4} & 0,4854 & 0,4459 & 0,3713 & 0,5920 & 0,5968 & 0,4441 & 0,5458 & 0,5227 & 0,4512\end{array}$

$\begin{array}{llllllllll}237,6 & 0,4817 & 0,4426 & 0,3685 & 0,5876 & 0,5923 & 0,4408 & 0,5418 & 0,5188 & 0,4479\end{array}$

$\begin{array}{llllllllll}237,8 & 0,4781 & 0,4393 & 0,3658 & 0,5832 & 0,5879 & 0,4375 & 0,537 & 0,5149 & 0,4445\end{array}$

$\begin{array}{llllllllll}238,0 & 0,4746 & 0,4360 & 0,3630 & 0,5789 & 0,5835 & 0,4342 & 0,5337 & 0,5111 & 0,4412\end{array}$ 
$\begin{array}{llllllllll}\text { Zeit } & \mathrm{P}_{1} & \mathrm{P}_{2} & \mathrm{P}_{3} & \mathrm{P}_{4} & \mathrm{P}_{5} & \mathrm{P}_{6} & \mathrm{P}_{7} & \mathrm{P}_{8} & \mathrm{P}_{9}\end{array}$

$\begin{array}{llllllllll}238,3 & 0,4710 & 0,4327 & 0,3603 & 0,5746 & 0,5791 & 0,4310 & 0,5297 & 0,5073 & 0,4379\end{array}$

$\begin{array}{llllllllll}238,5 & 0,4675 & 0,4295 & 0,3576 & 0,5703 & 0,5748 & 0,427 & 0,5257 & 0,5035 & 0,4346\end{array}$

$\begin{array}{llllllllll}238,7 & 0,4640 & 0,4263 & 0,3549 & 0,5660 & 0,5705 & 0,4245 & 0,5218 & 0,4997 & 0,4314\end{array}$

$\begin{array}{llllllllll}238,9 & 0,4605 & 0,4231 & 0,3523 & 0,5618 & 0,5663 & 0,4214 & 0,5179 & 0,4960 & 0,4281\end{array}$

$\begin{array}{llllllllll}239,1 & 0,4571 & 0,4199 & 0,3497 & 0,5576 & 0,5620 & 0,4182 & 0,5141 & 0,4923 & 0,4249\end{array}$

$\begin{array}{llllllllll}239,4 & 0,4537 & 0,4168 & 0,3471 & 0,5534 & 0,5578 & 0,4151 & 0,5102 & 0,4886 & 0,4218\end{array}$

$\begin{array}{llllllllll}239,6 & 0,4503 & 0,4137 & 0,3445 & 0,5493 & 0,5537 & 0,4120 & 0,5064 & 0,4850 & 0,4186\end{array}$

$\begin{array}{llllllllll}239,8 & 0,4469 & 0,4106 & 0,3419 & 0,5452 & 0,5495 & 0,4089 & 0,5026 & 0,4813 & 0,4155\end{array}$

$\begin{array}{llllllllll}240,0 & 0,4436 & 0,4075 & 0,3393 & 0,5411 & 0,5454 & 0,4059 & 0,4989 & 0,477 & 0,4124\end{array}$ 


\section{Danksagung}

Hiermit möchte ich all jenen recht herzlich danken, die zum Gelingen der vorliegenden Arbeit beigetragen haben:

Herrn Prof. Dr. Ir. Herman Van den Weghe $\left(\mathrm{FOSVWE}^{6}\right)$ für die wissenschaftliche Betreuung und das entgegengebrachte Vertrauen.

Herrn Prof. Dr.-Ing. Axel Munack $\left(\mathrm{FAL}^{7}\right)$ für die Übernahme des Korreferats und kritische Durchsicht dieser Arbeit.

Herrn Dr.-Ing. Karl-Heinz Krause (FAL) gilt mein besonderer Dank für die fachlich höchst kompetenten Diskussionen und Anregungen.

Herrn Dipl.-Ing. (FH) August-Wilhelm Zielstorff (FAL), dem unersetzlichen "Handbuch der Informatik", für seine unkomplizierte und immer freundliche Hilfestellung in zahlreichen Programmierstunden.

Herrn Stefan Linke (FAL) für die zahlreich durchgeführten Simulationsrechnungen und für die zur Verfügung gestellte Rechnerkapazität.

Herrn Dr.-Ing. Gerhard Jahns (FAL) für die Überarbeitung des englischen Summary.

Herrn Dr.-Ing. Hans-Joachim Müller, Herrn Dr. rer. nat. habil. Manfred Gläser, Herrn M. sc. Heiko Krehl, Herrn Dipl.-Ing. (FH) Bernd Möller sowie Herrn Dipl.-Ing. (FH) Ullrich Stollberg (ATB ${ }^{8}$ ) für die Versuchsdurchführung in den Praxisställen und fachgerechte Betreuung vor Ort.

Herrn Dipl.-Inform. Sandro Wefel $\left(\mathrm{MLU}^{9}\right)$ für die freundschaftliche Unterstützung bei der Bearbeitung des $\mathrm{LT}_{\mathrm{E}} \mathrm{X}$-Dokumentes zur Veröffentlichung der Dissertation im Internet.

\footnotetext{
${ }^{6}$ Forschungs- und Studienzentrum für Veredelungswirtschaft Weser-Ems der Georg-August-Universität Göttingen in Vechta

${ }^{7}$ Institut für Technologie und Biosystemtechnik der Bundesforschungsanstalt für Landwirtschaft in Braunschweig

${ }^{8}$ Abteilung Technik in der Tierhaltung im Agrartechnischen Institut Potsdam-Bornim

${ }^{9}$ Institut für Informatik der Martin-Luther-Universität Halle-Wittenberg
} 


\section{Lebenslauf}

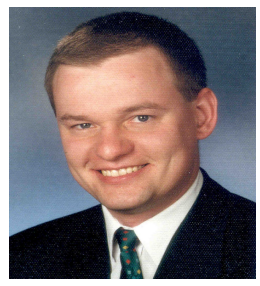

Name

Brehme

Vorname

Gunnar

Geburtsdatum

01.09.1971

Geburtsort

Halle/Saale

Familienstand

verheiratet

Kinder

1 Tochter

\section{Schulische Ausbildung}

Allgemeinbildende Polytechnische Oberschule Zörnigall

1978-1988

Erweiterte Oberschule Wittenberg (Abitur)

1988-1990

\section{Studium}

Praktisches Jahr bei Gerhard de Vries, Veenhusen bei Leer

1990-1991

Georg-August-Universität Göttingen, Fakultät für

1991-1996

Agrarwissenschaften, Fachrichtung Wirtschafts- und

Sozialwissenschaften des Landbaus (WiSoLa)

Abschluss als Diplomagraringenieur

\section{Berufstätigkeit}

Institut für Biosystemtechnik (BST) der

Bundesforschungsanstalt für Landwirtschaft (FAL),

Braunschweig-Völkenrode

wissenschaftlicher Mitarbeiter

Agrartechnisches Institut Potsdam-Bornim (ATB)

seit 1999

wissenschaftlicher Mitarbeiter

\section{Landwirtschaftliche Praktika}

LPG Tierproduktion Mühlanger bei Wittenberg

Geflügelinstitut Merbitz bei Halle/Saale

LPG Tierproduktion Gallin bei Wittenberg

LPG Tierproduktion Leetza bei Wittenberg 